\title{
HYDROLOGIC DATA FOR BLOCK ISLAND, RHODE ISLAND
}

By Emily Burns

U.S. GEOLOGICAL SURVEY

Open-File Report 92-155

Prepared in cooperation with the

TOWN OF NEW SHOREHAM, RHODE ISLAND

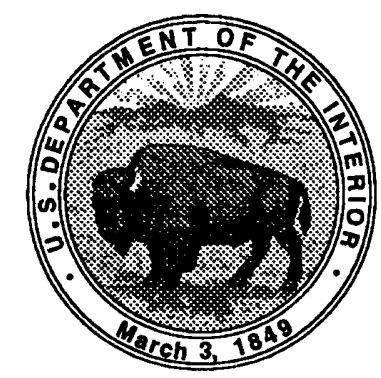

Providence, Rhode Island 1993 


\title{
U.S. DEPARTMENT OF THE INTERIOR \\ BRUCE BABBITT, Secretary
}

\author{
U.S. Geological Survey
}

Robert M. Hirsch, Acting Director

For additional information write to:

Chief, Rhode Island Subdistrict

U.S. Geological Survey

237 John O. Pastore Federal Bldg.

Providence, RI 02903-1720
Copies of this report can

be purchased from:

U.S. Geological Survey

Earth Science Information Center

Open-File Reports Section

Box 25286, MS 517

Federal Center

Denver, CO 80225 


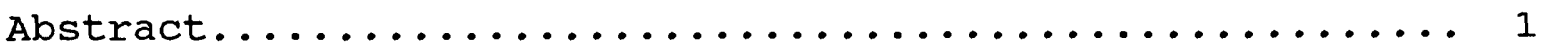

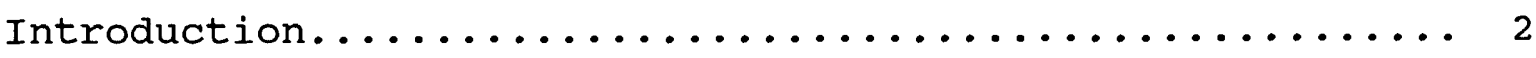

Purpose and scope...................... 3

Acknowledgements........................ 3

Description of hydrologic data................. 4

Numbering and location of data-collection sites........ 5

Data-collection methods..................... 6

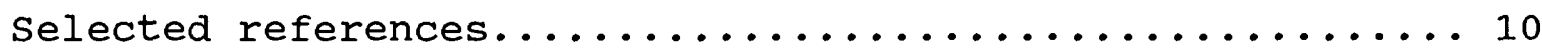

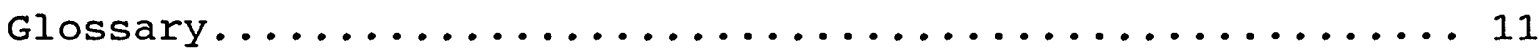

\section{ILIUSTRATIONS}

Plate 1. Map of Block Island showing location of data-collection sites............. in pocket

Page

Figure 1. Hydrographs showing ground-water levels at selected wells.................. 109 


\section{TABLES}

1. Description of selected wells, springs, and test

Page borings.......................... 16

2. Lithologic logs of selected wells and test borings........................... 52

3. Total monthly precipitation at selected stations (801-811) from October 1988 to December 1990... 98

4. Periodic water levels in selected ponds......... 99

5. Periodic water levels in selected wells........ 103

6. Chemical and physical analyses of precipitation at Sands Pond well field (station 807)......... 111

7. Chemical and physical analyses of water from

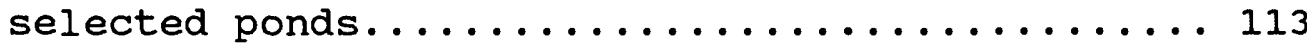

8. Chemical and physical analyses of water from selected wells and springs................ 116

9. Specific conductance of water from selected

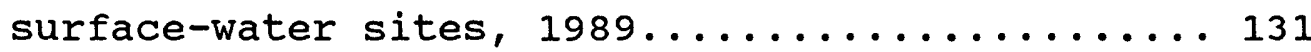

10. Specific conductance of water from selected

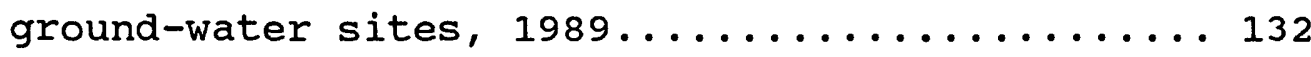

11-15. Rhode Island Department of Health analyses of

Block Island public drinking-water sources for:

11. Inorganics and physical characteristics...... 133

12. Total heavy metals................... 136

13. Chlorinated hydrocarbon pesticides and herbicides....................... 138

14. Chloroform and trihalomethanes............ 140

15. Radioactive constituents................ 141 


\section{CONVERSION FACTORS AND VERTICAL DATUM}

Multiply
By
To obtain

\section{Length}

inch (in.)

25.4

millimeter

foot (ft)

0.3048

meter

mile ( $\mathrm{mi}$ )

1.609 kilometer

\section{Area}

square mile $\left(\mathrm{mi}^{2}\right)$

2.590

square kilometer

\section{Volume}

gallon (gal)

$3.785 \quad$ liter

\section{F10w}

gallon per minute (gal/min) 0.06308 liter per second

\section{Temperature}

Temperature in degrees celsius $\left({ }^{\circ} \mathrm{C}\right)$ can be converted to degrees Fahrenheit $\left({ }_{F}\right)$ as follows:

$$
{ }^{\circ} \mathrm{F}=1.8\left({ }^{\circ} \mathrm{C}\right)+32
$$

Sea level: In this report, "sea level" refers to the National Geodetic Vertical Datum of 1929--a geodetic datum derived from a general adjustment of the first-order level nets of the United states and Canada. Formerly called Sea Level Datum of 1929 . 


\title{
By
}

\section{Emily Burns}

\begin{abstract}
This report was compiled as part of a study to assess the hydrogeology and the quality and quantity of fresh ground water on Block Island, Rhode Island. Hydrologic data were collected on Block Island during 1988-91. The data are presented in illustrations and tables. Data collected include precipitation, surfacewater, ground-water, lithologic, and well-construction and discharge information. Precipitation data include total monthly precipitation values from 11 rain gages and water-quality analyses of 14 precipitation samples from one station. Surfacewater data include water-level measurements at 12 ponds, waterquality data for five ponds, and field specific-conductance measurements at 56 surface-water sites (streams, ponds, and springs). Ground-water data include water-level measurements at 159 wells, water-quality data at 150 wells, and field specificconductance data at 52 wells. Lithologic logs for 375 wells and test borings, and construction and location data for 570 wells,
\end{abstract}


springs, and test borings are included. In addition, the data set contains data on water quality of water samples, collected by the Rhode Island Department of Health during 1976-91, from Fresh and Sands Ponds and from wells at the Block Island Water Company well field north of Sands Pond.

\section{INTRODUCTION}

The data in this report are published to assist state and local water-resources planners in managing water use on Block Island. The data were collected during 1988-91 by the U.S. Geological survey (USGS) as part of a cooperative study with the Town of New Shoreham (Block Island), Rhode Island. The purpose of the study was to assess the hydrogeology and the quality and quantity of fresh ground water on Block Island.

Block Island is about $10 \mathrm{mi}$ south of the Rhode Island coast. It is approximately $3.5 \mathrm{mi}$ wide, $7 \mathrm{mi}$ long, and $11 \mathrm{mi}^{2}$ in area. The island is composed of unconsolidated Pleistocene glacial and glaciofluvial deposits. Included in these deposits are blocks of older Cretaceous sediments (Kaye, 1960). All these deposits supply ground water to wells. Most residents have private water wells. The principle water supply of the town is sands Pond in the central part of the island; an adjoining well field is used as a backup supply. 


\section{Purpose and Scope}

This report provides data on (1) well location and construction, (2) the lithology of the island's sediments, (3) water levels in wells and ponds on the island, and (4) the amount of and the water quality of the island's precipitation, surface water, and ground water. It also describes the methods used to obtain these data.

\section{Acknowledgements}

The author wishes to thank all the property owners on Block Island who granted permission for mapping, water sampling, test drilling, and water-level measurements on their property. special thanks are due to Barbara Burak for assisting in the matching of several hundred driller's well records with town plat and lot numbers. Well drillers W. Gordon Goold and Rick Batchelder provided the USGS with lithologic logs and construction data from several hundred wells and shared their considerable knowledge of the island's ground-water resources. Henry Dupont and Robert Downie of the Block Island Water Company provided information on the town water supply and permitted field work at the water company well field. Laura Thompson, formerly of the University of Rhode Island, was very helpful in assisting with digitizing well and lot information. Norman Dahl, chairperson of the water Resources committee for the Town of New Shoreham, collected precipitation data and coordinated the collection of precipitation data by Elizabeth Breuer, Barbara Burak, 
Joseph Connolly, Ralph Derby, John Hobe, Henry Lemoine, Pam Littlefield, Sue Littlefield, Doug Michel, and Joan Salzberg.

\section{DESCRIPTION OF HYDROLOGIC DATA}

This report contains data on the location, construction, water level, and yield for 570 selected wells, springs and test borings (table 1); lithologic logs of 375 of the wells and test borings (table 2); monthly precipitation records from 11 precipitation-gaging stations (table 3); periodic water-level measurements at 12 ponds (table 4); periodic water levels in 21 USGS observation wells and 140 private wells (table 5); chemical and physical analyses of 15 precipitation samples from one gaging station (table 6); chemical and physical analyses of 9 surfacewater samples from 5 ponds (table 7); chemical and physical analyses of 150 ground-water samples from 97 wells and 8 springs (table 8); and field specific-conductance measurements for 29 ponds, 20 springs, 7 streams (table 9), and water samples from 52 wells (table 10). The locations of these data-collection sites are shown on plate 1. Also included are water-level hydrographs for 5 wells where water levels were recorded continuously (fig. 1).

The data presented in this report are from several sources. Data collected for this study were obtained during october 1988June 1991. In addition, data from an earlier usGs study of 
ground water on Block Island (Hansen and Schiner, 1964, tables 35) are included, as well as water-quality data collected by the Rhode Island Department of Health from Sands Pond and the Block Island water company public-supply wells during 1976-91 (tables 11-15). All well-description data and USGS water-quality data in this report, with the exception of the lithologic logs, are stored on the USGS's New England area Ground-Water site Information computer data base (GWSI). The Rhode Island Department of Health data are stored at the Rhode Island Department of Health.

\section{NUMBERING AND LOCATION \\ OF DATA-COLIECTION SITES}

Each well or test hole inventoried by the USGS in Rhode Island is assigned an alphanumeric code consisting of a 2-letter town designator followed by a one-letter site designator and a sequential local site number. The town designator for New Shoreham is $\mathrm{NH}$. Site designators used in this study are $W$ for well, $S$ for spring, and $B$ for boring.

Additional sites such as precipitation-gaging stations, pond staff gages, and surface-water-quality sampling sites were given sequential numbers, starting with 800 . Locations of datacollection sites are shown on Plate 1. 


\section{DATA-COLLECTION METHODS}

The locations of wells, springs, and other data-collection sites were determined with the aid of tax plat maps and house numbers assigned by the fire department. Each site was visited and its location plotted on a USGS topographic map at a scale of 1:24,000. The data-collection sites were transferred to a stable-base topographic map at a scale of 1:12,000. The latitude and longitude of each site was then determined using a digitizer.

Altitudes of land surface at most sites were estimated in the field using the USGS topographic map of Block Island. Because contours on these maps are accurate to only $\pm 5 \mathrm{ft}$, estimated altitudes have a similar accuracy. Altitudes of sites inventoried by Hansen and Schiner (1964, table 4) and some datacollection sites for this study were determined with an altimeter; the accuracy of those measurements is about $\pm 1 \mathrm{ft}$. Forty-three data-collection sites were tied to known USGS or U.S. Coast and Geodetic survey benchmarks with either an automatic level or a laser level. The altitudes are shown in table 1.

Well drillers on Block Island provided the USGS with construction data and lithologic logs for more than 500 private and public supply wells. The lithologic logs of 349 wells and borings are included in table 2. The logs from 15 USGS observation wells drilled during 1988-89 and 11 test borings drilled in 1960 are also included in table 2, for a total of 375 logs. 
Ten island residents measured precipitation after every rain or snowfall using USGS rain gages installed on their property. Precipitation samples from the well field north of Sands Pond were collected for chemical and physical analysis after storms with a wet-fall dry-fall collector installed by the USGS. Precipitation measurements from the U.S. National Weather Service weather station at the Block Island state Airport are also included in the data set (table 3 ).

Pond levels were read weekly from staff gages. The gages were 3-ft-long $\times 0.33-f t-w i d e$ steel plates, which were marked off in 0.01-ft intervals, and bolted to steel fenceposts driven into the pond. Altitude datums for the Fresh and Sands Pond gages were determined by leveling; altitudes of the other ponds vere determined from a topographic map. Each staff gage was leveled in to a temporary reference point and releveled to this point once a year to check for movement. Any changes in altitude so detected were applied to the data as datum corrections, beginning with the most recent date on which the pond was observed to be frozen. Pond-level data are provided in table 4.

Depth to water in wells was determined using either a steel tape, an electric tape, or an acoustic water-level meter. Dug wells were usually measured with a steel tape; wells with water levels greater than $50 \mathrm{ft}$ were measured either by electric tape or by an acoustic meter; and wells with water levels greater than $100 \mathrm{ft}$ were measured with the acoustic meter. Two measurements were made several minutes apart to ensure that the water level 
was stable. Accuracy of steel-tape measurements is $\pm 0.01 \mathrm{ft}$. Acoustic and electric-tape measurements are accurate to $\pm 0.1 \mathrm{ft}$. Water-level data are provided in tables 1 and 5 . These tables also contain water levels reported by drillers and well owners. The methods of measurement of these data are not known; thus their accuracy can not be assessed. Hourly water-level measurements were obtained at five wells using analog-digital recorders. These data are provided in figure 1.

Samples from the precipitation gage at Sands Pond were collected after storms and analyzed at the USGS National water Quality Laboratory in Arvada, Colorado. Water-quality data for precipitation samples are provided in table 6 .

Pond-water samples were collected during 1962-63 (Hansen and Schiner, 1964, table 3) and, for this study, during October 1988March 1989. Samples collected for this study were obtained by lowering a weighted tube into the pond, sealing the top, withdrawing the tube, and collecting the column of water contained in the tube. The length of the water column collected is included in table 7. No record is available of how the samples were collected in 1962 and 1963.

Ground-water samples were collected from springs, private wells, and observation wells over a 3-year period. Some shallow wells were sampled with a centrifugal pump and polyvinyl chloride (PVC) tubing. It is possible that some sample contamination of metals may have occurred because the water was in contact with 
the pump. other wells were sampled with a peristaltic pump and Teflon ${ }^{1}$ tubing. The centrifugal and peristaltic pumps may have aerated the sample. Wells with existing pumps were sampled at water taps prior to passing through water-treatment equipment. All wells were pumped until pH and specific conductance stabilized before a sample was collected. springs were sampled by holding sample bottles in the flow.

Samples were analyzed in the field for $\mathrm{pH}$, dissolved oxygen concentration, water temperature, and specific conductance. The following were analyzed at the USGS Providence office: alkalinity and bicarbonate alkalinity, using a digital titrator with 0.16-Normal sulfuric acid and a pH meter; and (2) chloride, for five ground-water samples collected during 1991, using a digital titrator, with the mercuric nitrate method. The USGS National Water Quality Laboratory in Arvada, Colorado, performed all other chemical and physical analyses on samples collected for this study. Water samples were packed in coolers and iced before shipment to the laboratory to keep them at less than $4{ }^{\circ} \mathrm{C}$. Results of laboratory and field analyses are provided in table 8 .

${ }^{1}$ Use of the trade name in this report is for identification purposes only and does not constitute endorsement by the U.S Geological survey. 
Field specific-conductance measurements were made in 1989 at wells where water-level measurements were made. Surface-water sites (ponds, springs, and streams) were measured for specific conductance during July 1989. Specific conductance data for surface-water samples are in provided table 9, and specific conductance data for ground-water samples are given in table 10.

The Rhode Island Department of Health samples public water supplies annually. They provided the USGS with water-quality data for Sands Pond, Fresh Pond, and several of the water company supply wells (tables 11-15).

\section{SELECTED REFERENCES}

Hansen, A.J., and Schiner, G.R., 1964, Ground water resources of Block Island, Rhode Island: Rhode Island Water Resources Coordinating Board Hydrologic Bulletin 14, 1 sheet, $35 \mathrm{p}$.

Hem, J.D., 1985, study and interpretation of the chemical characteristics of natural water (3d ed.): U.S. Geological Survey Water-Supply Paper 2254, 263 p.

Kaye, C.R., 1960, Surficial geology of the Kingston quadrangle Rhode Island: U.S. Geological Survey Bulletin 1071-I, 3 sheets, scale 1:24000, 55 p. 
Lohman, S.W., and others, 1972, Definitions of selected groundwater terms - revisions and conceptual refinements: U.S. Geological Survey Water-Supply Paper 1988, 15 p.

Moody, D.W., Carr, Jerry, Chase, E.B., and Paulson, R.W., compilers, 1986, National water summary 1986 - hydrologic events and ground-water quality: U.S. Geological survey Water-Supply Paper 2325, 560 p.

Rhode Island Department of Health, 1991, Rules and Regulations Pertaining to Public Drinking Water (R46-13-DWQ), $95 \mathrm{p}$.

\section{GLOSSARY}

Some definitions of terms used in this report were simplified for clarity.

AQUIFER: A formation, group of formations, or part of a formation that contains enough saturated, permeable material to yield significant quantities of water to wells or springs.

CRETACEOUS: A geological period that lasted from 135 to 65 million years ago. A geological deposit that formed during this time period is called a cretaceous deposit. 
GIACIAL: Pertaining to or influenced by the action of glaciers; formed or deposited by glacial action. Glacial sediments include till and stratified drift.

GLACIOFLUVIAL: Pertaining to or influenced by the action of glacial meltwater. Glaciofluvial sediments have been sorted into layers of different grain sizes by rivers of meltwater. They are often referred to as stratified drift.

GROUND WATER: In this report, water in the ground that is in the saturated zone and that contributes water to wells, springs, and ground-water runoff.

HARDPAN: A term commonly applied by New England well drillers to a glacial deposit that resists penetration by drilling equipment. The material commonly is till.

KAOLIN: A term found in some of the driller's logs in this report, where it presumably refers to white clay. Kaolin, or kaolinite, is a mineral which forms clay, on its own or more commonly with other clay minerals.

MICROGRAMS PER IITER (ug/L): A unit for expressing the concentration of chemical or physical constituents in a solution. Micrograms per liter represents the weight of a dissolved substance in micrograms per liter of water. (One microgram equals 0.001 milligrams.) Approximately equal to parts per billion for fresh (nonsaline) water. 
MILLIGRAMS PER LITER (mg/L): A unit for expressing the concentration of chemical or physical constituents in solution. Milligrams per liter represents the weight of a substance in milt.

1t4rams per liter of water. (One milligram equals 0.001 grams.) Approximately equal to parts per million for fresh (nonsaline) water.

pH: symbol denoting the concentration of hydrogen ions in a solution as a logarithm to negative base $10 ; \mathrm{pH}$ is an indication of the acidity of the solution. $\mathrm{pH}$ values range from 0 to 14 . A value of 7.0 indicates a neutral solution. Values greater than 7.0 indicate an alkaline (basic) solution; values less than 7.0 indicate an acidic solution. Because $\mathrm{pH}$ is a logarithmic value, a $\mathrm{pH}$ of 6.0 is 10 times more acidic (has 10 times more hydrogen ions) than a $\mathrm{pH}$ of 7.0 .

PICOCURIES PER LITER: A unit for expressing the concentration of radioactive constituents in solution. Picocuries per liter represents the radioactivity of a dissolved substance in picocuries per liter of water. A picocurie is one-trillionth $\left(1 \times 10^{-12}\right)$ of the amount of radioactivity represented by a curie. A curie is the amount of radioactivity that yields $3.7 \times 10^{10}$ radioactive disintegrations per second. A picocurie yields 2.22 disintegrations per minute.

PLEISTOCENE: A geological epoch that lasted from about 2 million years ago to about 10,000 years ago. A geological deposit that formed during this time period is called a Pleistocene deposit. 
SATURATED ZONE: The part of a formation or group of formations in which all voids are filled with water under pressure greater than atmospheric pressure; the zone beneath the water table.

SPECIFIC CONDUCTANCE: A measure of the ability of water to conduct an electrical current, expressed in microsiemens per centimeter at $25^{\circ} \mathrm{C}$. Specific conductance is related to the type and concentration of dissolved solids in the water. This relation is not constant; it varies with changes in the composition of the water.

STRATIFIED DRIFT: Unconsolidated sediment, such as gravel, sand, silt, and clay, that has been transported and sorted by glacial meltwater and deposited in layers, or strata, of similar grain size.

TILL: Unconsolidated sediment, such as gravel, sand, silt, and clay, that has been transported by glacial ice. Till is poorly to unsorted. It commonly is very compact and difficult to penetrate with drilling equipment. Locally it is called hardpan.

WATER TABLE: The water table is that surface in an unconfined aquifer at which the pressure is atmospheric. It is defined by the level at which water stands in wells that penetrate the ground just far enough to hold standing water. The level of the water table is influenced by rainfall, transpiration by plants, evaporation, natural discharges to surface-water bodies, and discharges to wells. 
Table 1.--Description of selected wells, springs, and test borings

STATION NUMBER: Local well number which is an alphanumeric code consisting of a two-letter town designator followed by a oneletter site designator and a sequential local site number. The town designator for New Shoreham is NH. Site designators are $W$ for well, $S$ for spring, and $B$ for boring.

LATITUDE and LONGITUDE: Values of latitude are in degrees, minutes, and seconds North latitude, and values of longitude are in degrees, minutes, and seconds West longitude.

ALTITUDE OF LAND SURFACE: Altitudes are expressed in feet above sea level. Altitudes determined from the map or by altimeter are given to the nearest foot. Leveled altitudes are given to the nearest hundredth of a foot. Due to mapping inaccuracies, at some sites altitudes determined from $\mathrm{Plate} 1$ do not match the altitudes listed in this table. Any site where the difference between the table and the map is greater than 10 feet has been footnoted.

METHOD ALTITUDE DETERMINED: A, altimeter; L, level; M, map.

METHOD CONSTRUCTED: A, air-rotary; B, bored or augered; C, cable tool; D, dug; H, hydraulic rotary; V, driven; $\mathrm{Z}$, other. 
Table 1.--Description of selected wells, springs, and test borings--Continued

DEPTH DRILLED: Total depth of hole, in feet below land surface. Depth drilled is equal to depth of well unless casing was pulled back for screening.

DEPTH OF WELL: Total depth of finished well, in feet below land surface.

TYPE OF FINISH: C, porous concrete; $O$, open end; $S$, screen; $\mathrm{W}$, walled; $\mathrm{Z}$, other.

OPEN INTERVAL: Interval where well screen is exposed to the aquifer material, in feet below land surface; bottom of open interval is finished depth of well.

WATER LEVEL: Water levels are given in feet below land surface. Levels measured by steel tape are given to the nearest hundredth of a foot. Levels measured by electric tape or acoustic meter are given to the nearest tenth of a foot. Estimates and reported levels are given to the nearest foot.

METHOD WATER LEVEL MEASURED: E, estimated, method unknown; R, reported; $S$, steel tape; $T$, electric tape; $\mathrm{Z}$, acoustic meter. Although $E$, estimated, and $R$, reported, are not measurement methods, these codes are given as methods in the GWSI database. 
Table 1.--Description of selected wells, springs and test borings--Continued

PRIMARY USE OF SITE: $O$, observation; $U$, unused; $W$, withdrawal; $\mathrm{z}$, destroyed.

PRIMARY USE OF WATER: C, commercial; F, fire; $\mathrm{H}$, domestic; $\mathrm{P}$, public supply; U, unused.

DISCHARGE: Pumping rate for well, in gallons per minute.

DRAWDOWN: Water-level decline in a well caused by pumping. Drawdown is in feet below static water level. Information is not available on how long wells were pumped prior to measurement of drawdown. Drawdown values do not necessarily represent stable drawdown conditions at the indicated discharge rate.

TYPE OF LOG AVAILABLE: Type of log available in table 2. D, driller's log; G, geologist's log. 
Table 1.--Description of selected wells, springs, and test borings

[-. or -, no data available; 00 in date columns indicates date unknown]

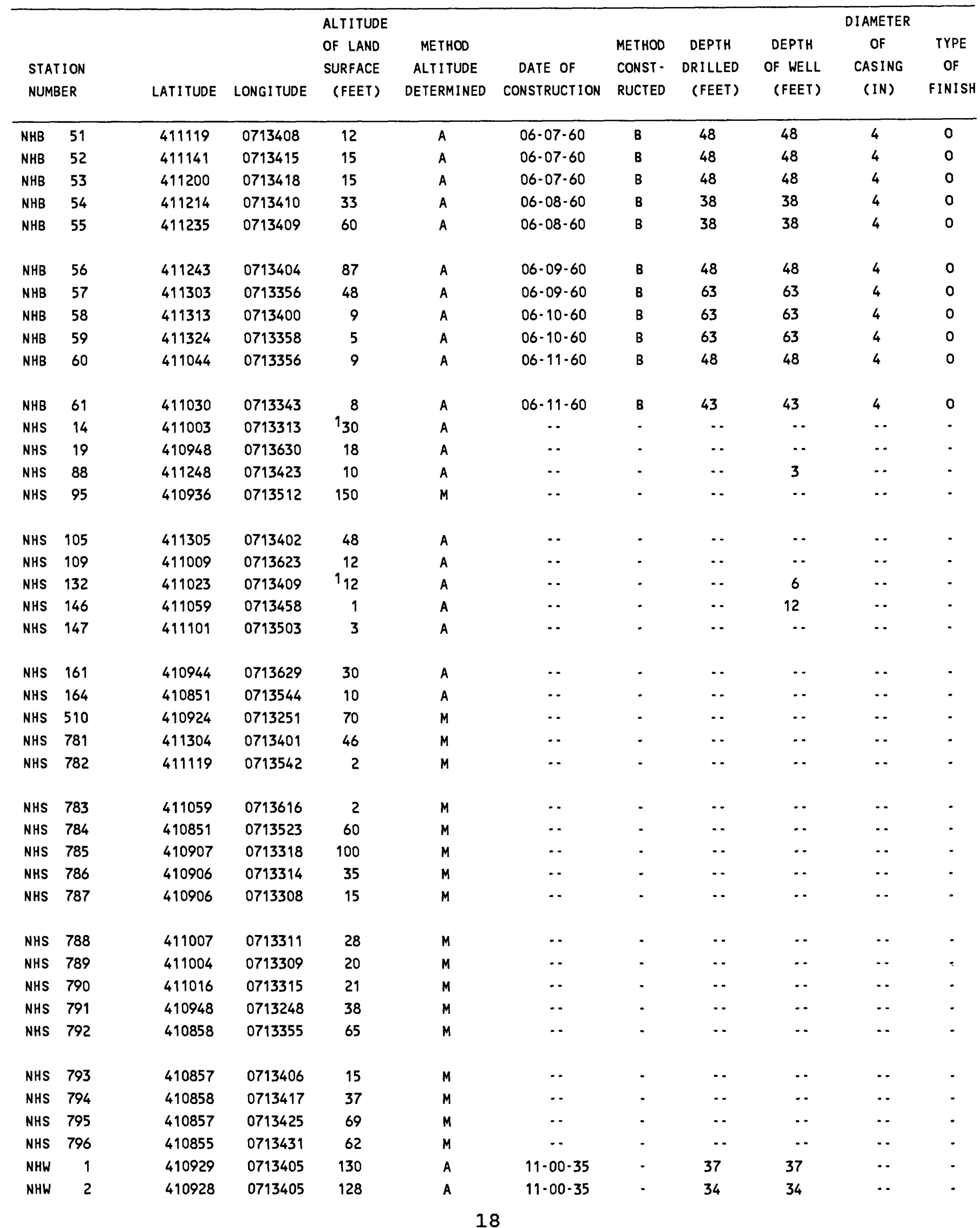


Table 1.--Description of selected wells, springs, and test borings--Continued

[-- or -, no data available; 00 in date columns indicates unknown]

\begin{tabular}{|c|c|c|c|c|c|c|c|c|c|c|c|}
\hline \multicolumn{2}{|c|}{$\begin{array}{l}\text { STATION } \\
\text { NUMBER }\end{array}$} & \multirow{2}{*}{$\begin{array}{c}\begin{array}{c}\text { TOP OF } \\
\text { OPEN } \\
\text { INTERVAL } \\
\text { (FEET) }\end{array} \\
.\end{array}$} & $\begin{array}{l}\text { BOTTOM OF } \\
\text { OPEN } \\
\text { INTERVAL } \\
\text { (FEET) }\end{array}$ & $\begin{array}{l}\text { WATER } \\
\text { LEVEL } \\
\text { (FEET) }\end{array}$ & $\begin{array}{l}\text { DATE } \\
\text { WATER } \\
\text { LEVEL } \\
\text { MEASURED }\end{array}$ & $\begin{array}{l}\text { METHOD } \\
\text { WATER } \\
\text { LEVEL } \\
\text { MEASURED }\end{array}$ & $\begin{array}{l}\text { PRIMARY } \\
\text { USE } \\
\text { OF } \\
\text { SITE }\end{array}$ & $\begin{array}{l}\text { PRIMARY } \\
\text { USE } \\
\text { OF } \\
\text { WATER }\end{array}$ & $\begin{array}{l}\text { DISCHARGE } \\
\text { (GPM) }\end{array}$ & $\begin{array}{l}\text { DRAWDOWN } \\
\text { (FEET) }\end{array}$ & \multirow{2}{*}{$\begin{array}{c}\text { TYPE } \\
\text { OF LOG } \\
\text { AVAILABLE } \\
G\end{array}$} \\
\hline NHB & 51 & & $\cdots$ & 11 & $06-07-60$ & $\mathrm{R}$ & $z$ & $u$ & $\cdots$ & $\cdots$ & \\
\hline NHB & 52 & $\cdots$ & .. & 5 & $06-07 \cdot 60$ & $\mathbf{R}$ & $z$ & $u$ & $\cdot \cdot$ & $\cdots$ & G \\
\hline NHB & 53 & -. & -. & 15 & $06-07 \cdot 60$ & $\mathbf{R}$ & $z$ & $u$ & $\cdots$ & $\cdots$ & G \\
\hline NHB & 54 & . & $\cdots$ & 31 & $06-08-60$ & $R$ & $z$ & $u$ & $\cdots$ & $\cdots$ & $G$ \\
\hline NHB & 55 & -. & -. & 32 & $06 \cdot 08-60$ & $\mathrm{R}$ & $z$ & $u$ & $\cdots$ & $\cdots$ & G \\
\hline NHB & 56 & .. & .. & $\cdots$ & .. & $R$ & $z$ & u & $\cdots$ & $\cdots$ & G \\
\hline NHB & 57 & -. & .. & 5 & $06-09 \cdot 60$ & $R$ & z & u & -. & -. & G \\
\hline NHB & 58 & .. & .. & 6 & $06-10-60$ & $R$ & $z$ & u & .. & . & $G$ \\
\hline NHB & 59 & .. & .. & 4 & $06 \cdot 10-60$ & $R$ & $z$ & $u$ & .. & .. & $G$ \\
\hline NHB & 60 & $\cdots$ & $\cdots$ & 8 & $06-11-60$ & $\mathrm{R}$ & $z$ & $u$ & -. & $\cdots$ & $G$ \\
\hline NHB & 61 & $\cdots$ & .. & 8 & $06-11 \cdot 60$ & $R$ & $z$ & $u$ & .. & 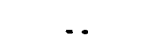 & $G$ \\
\hline NHS & 14 & -. & $\cdots$ & -. & . & $\cdot$ & - & $P$ & 36 & $\cdots$ & - \\
\hline NHS & 19 & -. & $\cdots$ & -. & $\cdots$ & - & - & $\cdot$ & 5 & .. & . \\
\hline NHS & 88 & $\cdots$ & $\cdots$ & $\cdots$ & -. & - & . & $H$ & 1.5 & .. & - \\
\hline NHS & 95 & .. & -. & -. & -. & $\cdot$ & . & $u$ & 2 & -. & - \\
\hline NHS & 105 & .. & .. & $\because$ & -. & - & . & H & .. & . & . \\
\hline NHS & 109 & .. & .. & $\cdots$ & . & . & - & $H$ & $\cdots$ & $\cdots$ & - \\
\hline NHS & 132 & -. & .. & 3.02 & $07-06-62$ & s & $\cdot$ & $H$ & 0.4 & . & - \\
\hline NHS & 146 & -. & $\cdots$ & .. & - & - & - & u & -. & . & - \\
\hline NHS & 147 & $\cdots$ & $\cdot \cdot$ & $\cdot \cdot$ & $\because$ & $\cdot$ & $\cdot$ & u & $\cdots$ & $\cdots$ & $\cdot$ \\
\hline NHS & 161 & $-\cdot$ & $\cdots$ & .. & .. & - & - & H & .. & .. & - \\
\hline NHS & 164 & . & $\cdot \cdot$ & $\cdots$ & $\cdots$ & - & - & H & 15 & $\cdots$ & - \\
\hline NHS & 510 & $\cdots$ & $\cdots$ & $\cdots$ & $\cdots$ & - & - & H & 7 & $\cdot \cdot$ & - \\
\hline NHS & 781 & -. & $\cdots$ & .. & .. & - & - & $u$ & $\cdots$ & $\cdots$ & - \\
\hline NHS & 782 & .. & -. & .. & $\cdots$ & - & $\cdot$ & u & -. & -. & $\cdot$ \\
\hline NHS & 783 & -. & -. & .. & .. & - & - & $u$ & .. & .. & - \\
\hline NHS & 784 & -. & $\cdots$ & $\cdots$ & $\cdots$ & $\cdot$ & - & $u$ & $\cdots$ & .. & - \\
\hline NHS & 785 & -. & $\cdots$ & $-\cdot$ & $\cdots$ & - & - & U & $\cdots$ & 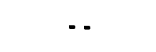 & - \\
\hline NHS & 786 & $\cdots$ & $\cdots$ & -. & $\cdots$ & - & - & U & $\cdots$ & $\cdots$ & - \\
\hline NHS & 787 & $\cdot \cdot$ & $\cdot \cdot$ & $\cdot \cdot$ & -. & $\cdot$ & - & $u$ & -. & . & $\cdot$ \\
\hline NHS & 788 & -. & . & $\cdots$ & $\cdots$ & - & - & $u$ & .. & . & . \\
\hline NHS & 789 & $\cdots$ & $\cdot \cdot$ & .. & .. & - & - & u & .. & .. & - \\
\hline NHS & 790 & $\cdots$ & $\cdots$ & $\cdots$ & $\cdots$ & . & - & u &.. & $\cdots$ & - \\
\hline NHS & 791 & .. & .. & -. & -. & $\cdot$ & $\cdot$ & $u$ & .. & .. & - \\
\hline NHS & 792 & -. & -. & . & . & $\cdot$ & - & $u$ & -. & . & - \\
\hline NHS & 793 & $-\cdot$ & .. & -. & .. & - & - & $u$ & .. & .. & - \\
\hline NHS & 794 & $\cdots$ & -. & $\cdots$ & -. & - & . & $u$ & -. & .. & . \\
\hline NHS & 795 & .. & -. & $\cdots$ & 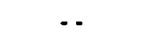 & $\cdot$ & $\cdot$ & $u$ & -. & $\cdots$ & $\cdot$ \\
\hline NHS & 796 & $\cdots$ & $\cdots$ & $\cdot \cdot$ & $\cdot \cdot$ & - & - & $u$ & -. & $\cdots$ & - \\
\hline NHW & 1 & $\cdots$ & $\cdots$ & 12.60 & $07 \cdot 22 \cdot 49$ & $s$ & $z$ & $u$ & 30 & .. & D \\
\hline NHW & 2 & $\cdots$ & -. & 12.74 & $07-22-49$ & s & $z$ & $u$ & 12 & $-\cdot$ & D \\
\hline
\end{tabular}


Table 1.--Description of selected wells, springs, and test borings--Continued

[-- or -, no data available; 00 in date columns indicates date unknown]

\begin{tabular}{|c|c|c|c|c|c|c|c|c|c|c|c|}
\hline & & & & ALTITUDE & & & & & & DIAMETER & \\
\hline & & & & OF LAND & METHOD & & METHOD & DEPTH & DEPTH & OF & TYPE \\
\hline STA & ION & & & SURFACE & ALTITUDE & DATE OF & CONST - & DRILLED & OF WELL & CASING & of \\
\hline NUM & & LATITUDE & LONGI TUDE & (FEET) & DETERMINED & CONSTRUCTION & RUCTED & (FEET) & (FEET) & (IN) & FINISH \\
\hline NHW & 3 & 410929 & 0713405 & 130 & A & $11-00-35$ & $\cdot$ & 37 & 37 & $\cdots$ & - \\
\hline NHW & 11 & 411031 & 0713414 & 6 & A & $10-00-43$ & C & 63 & 63 & 6 & - \\
\hline NHW & 12 & 411031 & 0713416 & 10 & A & $00-00-35$ & v & 52 & 52 & $\cdots$ & $\cdot$ \\
\hline NHW & 13 & 411031 & 0713415 & 10 & A & $09-00-35$ & - & 56 & 56 & $\cdots$ & - \\
\hline NHW & 15 & 410911 & 0713306 & 150 & $M$ & $00-00-35$ & c & 90 & 90 & 6 & - \\
\hline NHW & 17 & 411018 & 0713326 & 25 & A & $\cdots$ & D & 7 & 7 & $\cdots$ & w \\
\hline NHW & 18 & 410858 & 0713426 & 60 & A & $\cdots$ & D & 8 & 8 & $\cdots$ & W \\
\hline NHW & 31 & 411011 & 0713447 & 103 & A & $08-00-50$ & C & 155 & 155 & 6 & s \\
\hline NHW & 32 & 410928 & 0713405 & 130 & A & $07-00-50$ & C & 88 & 88 & 6 & $S$ \\
\hline NHW & 33 & 410931 & 0713403 & 131.25 & $L$ & $07-00-50$ & $c$ & 160 & 135 & 6 & $s$ \\
\hline & & & & & & $\cdots$ & - & & & $\cdots$ & - \\
\hline NHW & 34 & 410933 & 0713401 & 130.29 & L & $07-00-51$ & C & 107 & 107 & 6 & s \\
\hline & & & & & & $-\cdot$ & - & & & $\cdots$ & $\cdot$ \\
\hline & & & & & & $\cdots$ & - & & & $\cdots$ & - \\
\hline & & & & & & $\cdots$ & $\cdot$ & & & $\cdots$ & $\cdot$ \\
\hline NHW & 35 & 411105 & 0713405 & 9 & A & $05-00-54$ & C & 75 & 72 & 6 & $\cdot$ \\
\hline NHW & 36 & 411139 & 0713359 & 22 & A & $00-00-50$ & C & 40 & 40 & 4 & $s$ \\
\hline NHW & 37 & 411143 & 0713405 & 35 & A & $00-00-51$ & C & 62 & 49 & $\cdots$ & $s$ \\
\hline NHW & 38 & 411140 & 0713426 & 11 & A & $00 \cdot 00 \cdot 50$ & C & 30 & 30 & 4 & s \\
\hline NHW & 39 & 411259 & 0713410 & 68 & A & $00-00-50$ & C & 155 & 155 & $\cdots$ & - \\
\hline NHW & 40 & 411217 & 0713409 & 41 & A & $00-00-50$ & c & 61 & 61 & - & $s$ \\
\hline NHW & 41 & 411029 & 0713408 & 11 & A & $00-00-50$ & C & 109 & 109 & 4 & $s$ \\
\hline NHW & 42 & 411026 & 0713342 & ${ }^{1} 25$ & A & $00-00 \cdot 51$ & c & 96 & 96 & 4 & $s$ \\
\hline NHW & 43 & 411048 & 0713546 & 78 & A & $00-00-50$ & C & 105 & 105 & 4 & $s$ \\
\hline NHW & 44 & 411046 & 0713550 & 107 & A & $00-00-50$ & C & 139 & 139 & 4 & s \\
\hline NHW & 45 & 411049 & 0713525 & 85 & A & $00-00-50$ & C & 143 & 143 & 6 & 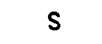 \\
\hline NHW & 46 & 411104 & 0713538 & 169 & A & $00-00-50$ & C & 88 & 88 & 4 & $s$ \\
\hline NHW & 47 & 411050 & 0713430 & 20 & A & $00-00-50$ & C & 38 & 38 & 6 & $\mathrm{~s}$ \\
\hline NHW & 48 & 410912 & 0713307 & 150 & M & $00 \cdot 00-50$ & C & 178 & 178 & 6 & $s$ \\
\hline NHW & 49 & 411042 & 0713413 & 17 & A & $06-16-55$ & C & 36 & 35 & 6 & S \\
\hline NHW & 50 & 411248 & 0713408 & 90 & A & $07-20-56$ & C & 233 & 233 & $\cdots$ & - \\
\hline NHW & 62 & 411250 & 0713359 & 111 & A & $00 \cdot 00-60$ & c & 316 & 270 & $\cdots$ & $\cdot$ \\
\hline NHW & 63 & 411325 & 0713343 & 44 & A & $00-00-56$ & C & 80 & 80 & $\cdots$ & - \\
\hline NHW & 64 & 411217 & 0713409 & 41 & A & $\cdots$ & C & 65 & 65 & 6 & $\cdot$ \\
\hline NHW & 65 & 411022 & 0713354 & $1_{40}$ & A & $\cdots$ & C & 85 & 85 & 6 & - \\
\hline NHW & 66 & 410949 & 0713604 & 88 & A & $00-00-55$ & C & 80 & 80 & 6 & s \\
\hline NHW & 67 & 410926 & 0713300 & 119 & A & $00-00-58$ & c & 240 & 240 & $\cdots$ & $s$ \\
\hline NHW & 68 & 410917 & 0713314 & 145 & A & $00-00-59$ & c & 145 & 145 & $\cdots$ & s \\
\hline NHW & 69 & 410943 & 0713250 & 66 & A & $\cdots$ & C & 100 & 100 & 6 & $\cdot$ \\
\hline NHW & 70 & 411022 & 0713444 & 44 & A & $00-00-57$ & C & 50 & 50 & 6 & $\cdot$ \\
\hline
\end{tabular}


Table 1.--Description of selected wells, springs, and test borings--Continued

[* or -, no data available; 00 in date columns indicates unknown]

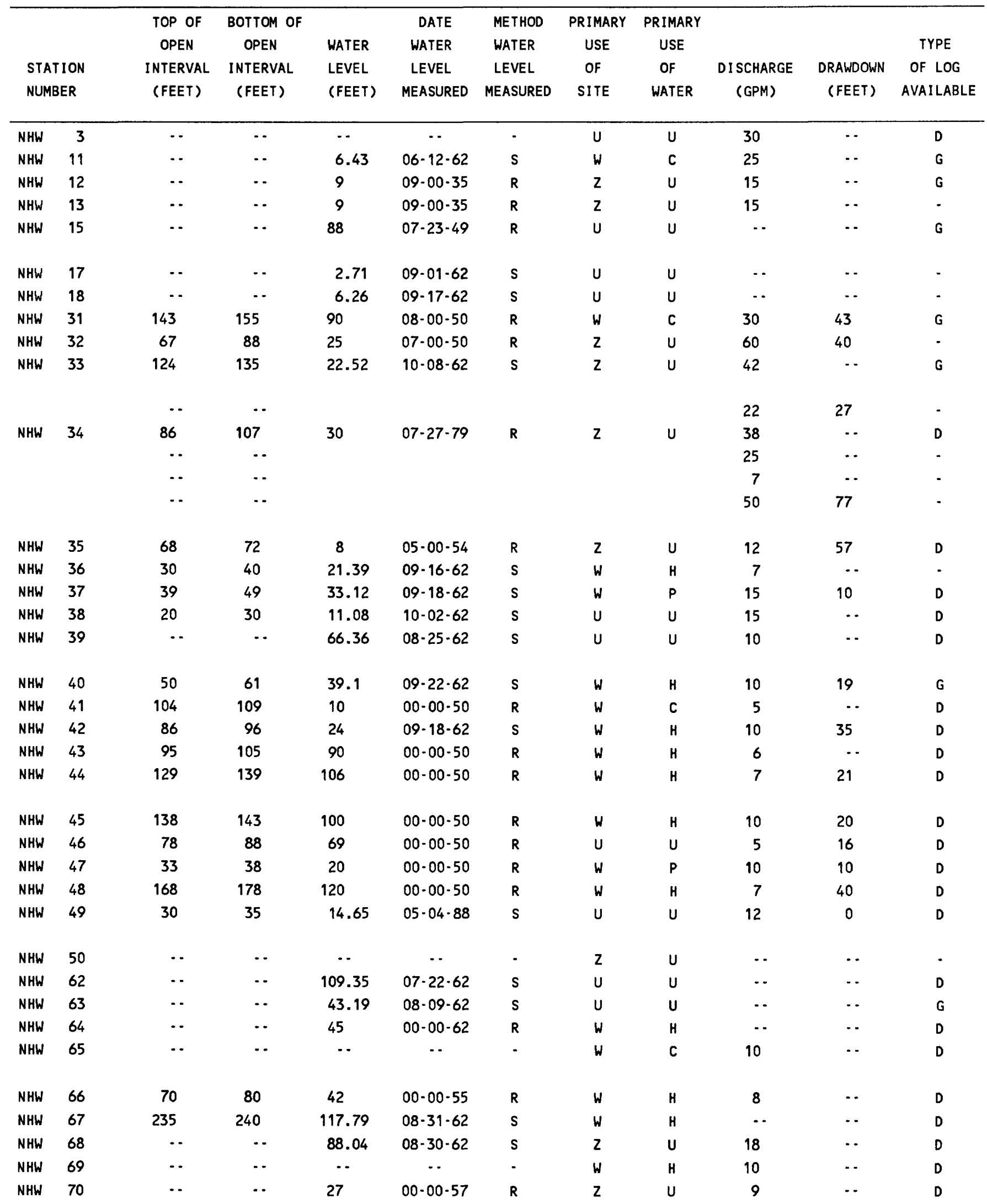


Table 1.--Description of selected wells, springs, and test borings--Continued

$$
\text { [.- or } \because \text {, no data available; } 00 \text { in date columns indicates date unknown] }
$$

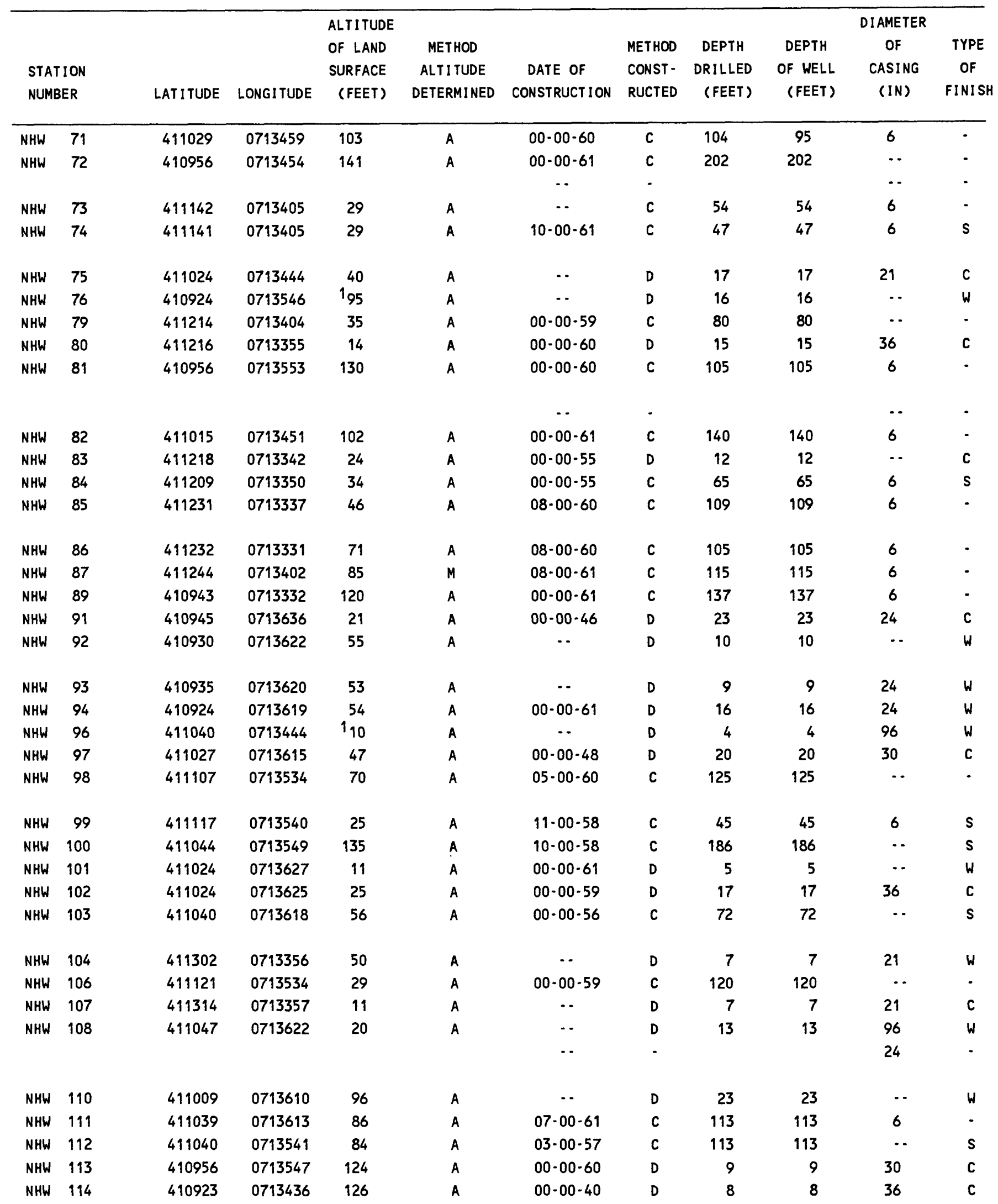


Table 1.--Description of selected wells, springs, and test borings--Cont inued

[-- or -, no data available; 00 in date columns indicates unknown]

\begin{tabular}{|c|c|c|c|c|c|c|c|c|c|c|c|}
\hline \multicolumn{2}{|c|}{$\begin{array}{l}\text { STATION } \\
\text { NUMBER }\end{array}$} & $\begin{array}{l}\text { TOP OF } \\
\text { OPEN } \\
\text { INTERVAL } \\
\text { (FEET) }\end{array}$ & $\begin{array}{l}\text { BOTTOM OF } \\
\text { OPEN } \\
\text { INTERVAL } \\
\text { (FEET) }\end{array}$ & $\begin{array}{l}\text { WATER } \\
\text { LEVEL } \\
\text { (FEET) }\end{array}$ & $\begin{array}{l}\text { DATE } \\
\text { WATER } \\
\text { LEVEL } \\
\text { MEASURED }\end{array}$ & $\begin{array}{l}\text { METHOD } \\
\text { WATER } \\
\text { LEVEL } \\
\text { MEASURED }\end{array}$ & $\begin{array}{l}\text { PRIMARY } \\
\text { USE } \\
\text { OF } \\
\text { SITE }\end{array}$ & $\begin{array}{l}\text { PRIMARY } \\
\text { USE } \\
\text { OF } \\
\text { WATER }\end{array}$ & $\begin{array}{c}\text { DISCHARGE } \\
\text { (GPM) }\end{array}$ & $\begin{array}{l}\text { DRAWDOWN } \\
\text { (FEET) }\end{array}$ & $\begin{array}{c}\text { TYPE } \\
\text { OF LOG } \\
\text { AVAILABLE }\end{array}$ \\
\hline NHW & 71 & -. & -- & 83.07 & $08-20-62$ & s & W & H & .. & -. & D \\
\hline \multirow[t]{2}{*}{ NHW } & 72 &  & $\cdots$ & 122.85 & $10-02 \cdot 62$ & s & $w$ & H & 10 & 30 & $\cdot$ \\
\hline & & $\cdots$ & $\cdots$ & & & & & & 10 & 30 & - \\
\hline NHW & 73 & $\cdots$ & $\cdots$ & $\cdot \cdot$ & -. & - & W & $\mathbf{P}$ & $\cdots$ & -. & - \\
\hline NHW & 74 & 42 & 47 & 28.08 & $09-27-62$ & s & $\mathbf{u}$ & $u$ & 12 & 11 & $\cdot$ \\
\hline NHW & 75 & $\cdots$ & $\cdots$ & 4.40 & $04-14-88$ & s & 0 & $u$ & $\cdots$ & $\cdots$ & G \\
\hline NHW & 76 & $\cdots$ & $\cdots$ & 12.83 & $10-06-62$ & $\mathbf{s}$ & $u$ & $u$ & -. & -. & G \\
\hline NHW & 79 & $\cdots$ & $\cdots$ & 33.60 & $09 \cdot 22 \cdot 62$ & $\mathbf{s}$ & W & $H$ & 12 & $\cdot \cdot$ & - \\
\hline NHW & 80 & $\cdots$ & -. & 10.18 & $03-11-88$ & $\mathbf{s}$ & W & H & -. & -. & - \\
\hline \multirow[t]{2}{*}{ NHW } & 81 & $\cdots$ & $\cdots$ & 91.60 & $09 \cdot 20 \cdot 62$ & $\mathbf{s}$ & W & H & 5 & $\cdot \cdot$ & D \\
\hline & & $\cdot \cdot$ & $-\cdot$ & & & & & & 5 & $\cdots$ & - \\
\hline NHW & 82 & -. & -. & 90 & $02-00-61$ & $\mathbf{R}$ & $\omega$ & H & 8 & 30 & - \\
\hline NHW & 83 & $\cdots$ & $\cdots$ & 11 & $08-18.62$ & $E$ & $w$ & H & $\cdots$ & $\cdots$ & $\cdot$ \\
\hline NHW & 84 & 58 & 65 & 32.67 & $09-22-62$ & s & $u$ & $u$ & 10 & 0 & $\cdot$ \\
\hline NHW & 85 & $\cdots$ & $\cdots$ & 45.08 & $08-29-62$ & s & $w$ & H & 8 & $\cdots$ & $\cdot$ \\
\hline NHW & 86 & $\cdot \cdot$ & $\cdots$ & 70 & $08-00-60$ & $R$ & $u$ & $u$ & 10 & 10 & $\cdot$ \\
\hline NHW & 87 & $\cdots$ & -. & 65 & $08-00-61$ & $R$ & $w$ & H & 8 & 20 & - \\
\hline NHW & 89 & -. & $\cdots$ & 101 & $00-00-61$ & $R$ & $w$ & H & 8 & 19 & - \\
\hline NHW & 91 & 23 & 23 & 21.0 & $03-18-88$ & s & w & H & .. & .. & G \\
\hline NHW & 92 & $\cdots$ & $\cdots$ & 6.40 & $09 \cdot 03 \cdot 62$ & s & $u$ & U & $\cdots$ & -. & $\cdot$ \\
\hline NHW & 93 & $\cdots$ & $\cdots$ & 6.82 & $09-02-62$ & s & $u$ & $u$ & -. & .. & - \\
\hline NHW & 94 & $-\cdot$ & .. & 7.47 & $06-22-88$ & s & $w$ & H & $-\cdot$ & $\cdots$ & - \\
\hline NHW & 96 & $-\cdot$ & $\cdots$ & 0.72 & $05-04-88$ & s & $w$ & H & $\cdots$ & -. & G \\
\hline NHW & 97 & $\cdots$ & $\cdots$ & 10.98 & $09-03-62$ & s & $w$ & H & -. & $\cdots$ & G \\
\hline NHW & 98 & -. & $\cdots$ & 68.74 & $09-20-62$ & s & $w$ & H & 10 & 20 & $\cdot$ \\
\hline NHW & 99 & 39 & 45 & 13.50 & $09-20-62$ & s & $w$ & H & 12 & 26 & $\cdot$ \\
\hline NHW & 100 & 143 & 186 & 132.16 & $10-08.62$ & s & $w$ & $H$ & 10 & 20 & - \\
\hline NHW & 101 & $\cdots$ & $\cdots$ & 3.12 & $09-03-62$ & s & 2 & $u$ & $\cdots$ & $\cdots$ & G \\
\hline NHW & 102 & $\cdots$ & $-\cdot$ & 14.06 & $09-03-62$ & s & $w$ & H & $\cdots$ & $\cdots$ & G \\
\hline NHW & 103 & 57 & 72 & 54.65 & $09-22-62$ & s & 2 & $u$ & 10 & 12 & $\cdot$ \\
\hline NHW & 104 & -. & .. & 1.8 & $03 \cdot 24 \cdot 88$ & s & $u$ & $u$ & $\cdots$ & .. & - \\
\hline NHW & 106 & $\cdots$ & -. & 28.14 & $10-04-62$ & $s$ & W & $H$ & 10 & $\cdots$ & $\cdot$ \\
\hline NHW & 107 & $\cdots$ & -. & 5.46 & $05-03-88$ & s & $w$ & H & $\cdots$ & $\cdots$ & - \\
\hline \multirow[t]{2}{*}{ NHW } & 108 & $\cdot \cdot$ & $\cdots$ & 9.50 & $08 \cdot 16 \cdot 62$ & s & 2 & $u$ & 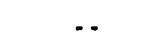 & -. & G \\
\hline & & -. & $\cdot-$ & & & & & & -. & $\cdots$ & $\cdot$ \\
\hline NHW & 110 & -. & $\cdots$ & 22.36 & $10-06-62$ & s & $w$ & H & .. & .. & - \\
\hline NHW & 111 & $-\cdot$ & $\cdots$ & 84.05 & $09-21 \cdot 62$ & $s$ & $w$ & $H$ & 8 & 16 & - \\
\hline NHW & 112 & 108 & 113 & 82 & $03-00-57$ & $R$ & $w$ & H & 12 & .. & $\cdot$ \\
\hline NHW & 113 & $\cdots$ & -. & 2.40 & $03-10-88$ & s & $w$ & $H$ & -. & -. & - \\
\hline NHW & 114 & -. & $-\cdot$ & 4.63 & $09 \cdot 06-62$ & s & $w$ & H & -. & $\cdots$ & - \\
\hline
\end{tabular}


Table 1.--Description of selected wells, springs, and test borings--Continued

[.- or -, no data available; 00 in date columns indicates date unknown]

\begin{tabular}{|c|c|c|c|c|c|c|c|c|c|c|c|}
\hline & & & & ALTI TUDE & & & & & & DIAMETER & \\
\hline & & & & OF LAND & METHOD & & METHOD & DEPTH & DEPTH & OF & TYPE \\
\hline $\begin{array}{l}\text { STAT } \\
\text { NUME }\end{array}$ & $\begin{array}{l}\text { TION } \\
\text { BER }\end{array}$ & LATI TUDE & LONGI TUDE & SURFACE & $\begin{array}{l}\text { ALTI I TUDE } \\
\text { DETERMINED }\end{array}$ & $\begin{array}{l}\text { DATE OF } \\
\text { CONSTRUCTION }\end{array}$ & CONST - & DRILLED & OF WELL & CASING & $\begin{array}{c}\text { OF } \\
\text { FINISH }\end{array}$ \\
\hline NHW & 115 & 410909 & 0713444 & 98 & A & $\cdots$ & D & 6 & 6 & $\cdots$ & $W$ \\
\hline NHW & 116 & 410938 & 0713304 & 90 & A & $\cdots$ & D & 13 & 13 & $\cdots$ & c \\
\hline NHW & 117 & 410937 & 0713312 & 118 & A & $00-00 \cdot 57$ & D & 8 & 8 & 21 & c \\
\hline NHW & 118 & 410934 & 0713256 & 115 & M & $03 \cdot 00-57$ & c & 193 & 193 & 6 & $\mathbf{s}$ \\
\hline NHW & 119 & 410918 & 0713315 & 146 & A & $00 \cdot 00 \cdot 54$ & D & 15 & 15 & $\cdots$ & c \\
\hline NHW & 120 & 410920 & 0713313 & 150 & A & $00-00 \cdot 61$ & D & 15 & 15 & $\cdots$ & $c$ \\
\hline NHW & 121 & 410948 & 0713259 & 44 & A & -. & D & 10 & 10 & $\cdots$ & $w$ \\
\hline NHW & 122 & 410954 & 0713336 & 95 & M & - & D & 7 & 7 & 21 & c \\
\hline NHW & 123 & 411002 & 0713621 & 24 & A & $00.00 \cdot 60$ & c & 68 & 68 & $\cdots$ & - \\
\hline NHW & 124 & 411023 & 0713348 & $1_{19}$ & A & $00-00-40$ & $D$ & 15 & 15 & -. & c \\
\hline NHW & 125 & 411023 & 0713346 & $1_{17}$ & A & $00 \cdot 00 \cdot 45$ & D & 14 & 14 & 18 & $w$ \\
\hline NHW & 126 & 411020 & 0713349 & 43 & A & -. & D & 23 & 23 & $\cdots$ & $w$ \\
\hline NHW & 127 & 411010 & 0713402 & 50 & A & $08 \cdot 00-56$ & c & 48 & 48 & 6 & $\cdot$ \\
\hline NHW & 128 & 411010 & 0713403 & 53 & A & $08 \cdot 00 \cdot 60$ & c & 86 & 86 & 6 & - \\
\hline & & & & & & .. & - & 86 & 86 & $\cdots$ & - \\
\hline NHW & 129 & 411017 & 0713403 & 46 & A & $00-00-59$ & c & 131 & 131 & 6 & - \\
\hline NHW & 130 & 411021 & 0713357 & 26 & A & $00-00-59$ & c & 55 & 55 & $\cdots$ & - \\
\hline NHW & 131 & 411019 & 0713405 & 16 & A & $00 \cdot 00 \cdot 20$ & D & 4 & 4 & 24 & w \\
\hline NHW & 133 & 411014 & 0713439 & 86 & A & $11-00-58$ & c & 130 & 130 & $-\cdot$ & $s$ \\
\hline NHW & 134 & 411019 & 0713358 & 20 & A & $00-00-56$ & c & 87 & 87 & $\cdots$ & - \\
\hline NHW & 135 & 411025 & 0713355 & 12 & A & -. & D & 18 & 18 & 36 & c \\
\hline NHW & 136 & 411016 & 0713351 & 32 & A & $05-00 \cdot 58$ & $c$ & 97 & 97 & $-\cdot$ & $\cdot$ \\
\hline NHW & 137 & 411016 & 0713403 & 51 & A & $\cdot-$ & $D$ & 21 & 21 & 18 & c \\
\hline NHW & 138 & 410956 & 0713353 & 67 & A & $00-00-57$ & $D$ & 7 & 7 & 24 & c \\
\hline NHW & 139 & 410954 & 0713352 & 68 & A & $00-00-15$ & D & 7 & 7 & 24 & $w$ \\
\hline NHW & 140 & 410955 & 0713353 & 66 & A & $-\cdot$ & $D$ & 6 & 6 & 24 & $w$ \\
\hline NHW & 142 & 411219 & 0713411 & 42 & A & $00-00-15$ & 0 & 41 & 41 & 21 & c \\
\hline NHW & 143 & 411148 & 0713417 & 28 & A & $05 \cdot 00-60$ & C & 71 & 71 & -. & - \\
\hline & & & & & & -. & - & & & $\cdots$ & $\cdot$ \\
\hline NHW & 144 & 411108 & 0713414 & 19 & A & $00-00-55$ & D & 24 & 24 & 30 & c \\
\hline NHW & 145 & 411106 & 0713411 & 6 & A & $00-00-45$ & D & 10 & 10 & .. & $w$ \\
\hline NHW & 148 & 411025 & 0713435 & 39 & A & $00-00-57$ & v & 19 & 19 & 4 & - \\
\hline NHW & 149 & 411008 & 0713326 & 73 & A & . & D & 15 & 15 & 30 & c \\
\hline NHW & 150 & 411206 & 0713345 & 24 & A & .. & v & 31 & 31 & 2.5 & $z$ \\
\hline NHW & 151 & 411023 & 0713356 & 10 & A & $00-00-47$ & D & 14 & 14 & $\cdots$ & c \\
\hline NHW & 152 & 411033 & 0713444 & 54 & A & $00-00.52$ & D & 26 & 26 & 36 & $c$ \\
\hline NHW & 153 & 410955 & 0713259 & 31 & A & $00-00-43$ & D & 23 & 23 & 30 & $c$ \\
\hline NHW & 154 & 410943 & 0713249 & 62 & A & $00 \cdot 00 \cdot 50$ & D & 21 & 20 & 36 & c \\
\hline NHW & 155 & 411018 & 0713357 & 21 & A & $\cdots$ & D & 11 & 11 & $\cdots$ & c \\
\hline
\end{tabular}


Table 1.--Description of selected wells, springs, and test borings--Continued

[-. or -, no data available; 00 in date columns indicates unknown]

\begin{tabular}{|c|c|c|c|c|c|c|c|c|c|c|c|}
\hline \multicolumn{2}{|c|}{$\begin{array}{l}\text { STATION } \\
\text { NUMBER }\end{array}$} & \multirow{2}{*}{$\begin{array}{c}\begin{array}{c}\text { TOP OF } \\
\text { OPEN } \\
\text { INTERVAL } \\
\text { (FEET) }\end{array} \\
\cdot .\end{array}$} & $\begin{array}{l}\text { BOTTOM OF } \\
\text { OPEN } \\
\text { INTERVAL } \\
\text { (FEET) }\end{array}$ & $\begin{array}{l}\text { WATER } \\
\text { LEVEL } \\
\text { (FEET) }\end{array}$ & $\begin{array}{c}\text { DATE } \\
\text { WATER } \\
\text { LEVEL } \\
\text { MEASURED }\end{array}$ & $\begin{array}{l}\text { METHOD } \\
\text { WATER } \\
\text { LEVEL } \\
\text { MEASURED }\end{array}$ & $\begin{array}{l}\text { PRIMARY } \\
\text { USE } \\
\text { OF } \\
\text { SITE }\end{array}$ & $\begin{array}{l}\text { PRIMARY } \\
\text { USE } \\
\text { OF } \\
\text { WATER }\end{array}$ & $\begin{array}{l}\text { DISCHARGE } \\
\text { (GPM) }\end{array}$ & $\begin{array}{l}\text { DRAWDOWN } \\
\text { (FEET) }\end{array}$ & $\begin{array}{c}\text { TYPE } \\
\text { OF LOG } \\
\text { AVAILABLE }\end{array}$ \\
\hline NHW & 115 & & $\cdots$ & 2.31 & $09-06-62$ & $s$ & w & H & $\cdots$ & -. & - \\
\hline NHW & 116 & $\cdots$ & -. & 6.00 & $08-13-62$ & $s$ & W & H & $\cdots$ & $\cdots$ & - \\
\hline NHW & 117 & $\cdots$ & $\cdots$ & 3.18 & $07-04-62$ & s & $u$ & $u$ & $\cdots$ & $\cdots$ & - \\
\hline NHW & 118 & 187 & 193 & 100.84 & $10-02-62$ & $s$ & w & H & 10 & 60 & - \\
\hline NHW & 119 & $\cdots$ & $\cdots$ & 13.75 & $09-01-62$ & $s$ & u & $u$ & $\cdots$ & $\cdots$ & G \\
\hline NHW & 120 & -. & $\cdots$ & 10.06 & $07-04-62$ & $s$ & $z$ & u & .. & .. & G \\
\hline NHW & 121 & 10 & -. & 6.73 & $08-05-62$ & s & $u$ & $u$ & - & -. & - \\
\hline NHW & 122 & $\cdots$ & $\cdots$ & 3.25 & $09-06-62$ & $s$ & $w$ & H & $\cdots$ & -. & - \\
\hline NHW & 123 & $\cdots$ & $-\cdot$ & 12.19 & $10-10-62$ & $s$ & $w$ & H & 5 & -. & - \\
\hline NHW & 124 & $\cdots$ & $\cdots$ & 12.69 & $07-05-62$ & $s$ & $u$ & $u$ & $\cdots$ & $\cdots$ & $\cdot$ \\
\hline NHW & 125 & $\cdots$ & - & 7.3 & $03-30-88$ & $s$ & $U$ & u & -. & -. & . \\
\hline NHW & 126 & .. & -. & 22.33 & $09-01.62$ & $s$ & $w$ & H & -. & .. & - \\
\hline \multirow[t]{2}{*}{ NHW } & 127 & $\cdots$ & $\cdots$ & 20 & $08-00-56$ & $R$ & U & $u$ & 9 & 25 & - \\
\hline & & $\cdot \cdot$ & -. & & & & & & 2 & .. & $\cdot$ \\
\hline NHW & 128 & $\cdots$ & $\cdot \cdot$ & 30 & $08.00 \cdot 60$ & $\mathrm{R}$ & $w$ & H & 6 & -. & - \\
\hline NHW & 128 & .. & -. & 30 & $08-00 \cdot 60$ & $R$ & $w$ & H & 6 & .. & . \\
\hline NHW & 129 & $\cdots$ & -. & 31 & $00-00-59$ & $R$ & $w$ & H & 20 & .. & - \\
\hline NHW & 130 & $\cdots$ & $\cdots$ & 25.41 & $09-22.62$ & $s$ & $u$ & $u$ & 8 & .. & - \\
\hline NHW & 131 & $\cdots$ & -. & 2.3 & $03-30 \cdot 88$ & $T$ & $w$ & H & . & .. & - \\
\hline NHW & 133 & 108 & 130 & 71.98 & $08-30-62$ & $s$ & $w$ & $H$ & 12 & 35 & - \\
\hline NHW & 134 & - & -. & 18 & $00-00-56$ & $R$ & $w$ & H & 10 & $\cdots$ & - \\
\hline NHW & 135 & - & -. & 10.62 & $09-01-62$ & $\mathrm{~s}$ & $w$ & H & .. & .. & - \\
\hline NHW & 136 & $\cdots$ & -. & 31.33 & $09-10-62$ & $\mathrm{~s}$ & $w$ & P & 16 & -. & - \\
\hline NHW & 137 & $\cdots$ & $\cdots$ & 15.92 & $10-06-62$ & s & $w$ & $P$ & .. & . & - \\
\hline NHW & 138 & $\cdots$ & $-\cdot$ & 3.60 & $07-07-62$ & $s$ & w & - & .. & .. & - \\
\hline NHW & 139 & $\cdots$ & .. & 2.12 & $07-07-62$ & $s$ & $w$ & $H$ & .. & -. & - \\
\hline NHW & 140 & $\cdots$ & -. & 1.65 & $07-07-62$ & s & w & H & .. & .. & - \\
\hline NHW & 142 & -. & .. & 40.57 & $09-05.62$ & $\mathrm{~s}$ & w & H & .. & .. & - \\
\hline \multirow[t]{2}{*}{ NHW } & 143 & -. & $\cdots$ & 26.84 & $10-10-62$ & s & $w$ & H & 8 & 12 & $\cdot$ \\
\hline & & $\cdots$ & -. & & & & & & 8 & 12 & - \\
\hline NHW & 144 & -. & .. & 18.01 & $09-05-62$ & $s$ & $w$ & H & .. & .. & - \\
\hline NHW & 145 & $\cdots$ & $\cdots$ & 4.97 & $07-09-62$ & $s$ & $w$ & $H$ & .. & . & . \\
\hline NHW & 148 & -. & $\cdots$ & 12.27 & $09-01-62$ & $s$ & $w$ & H & 5 & 2 & G \\
\hline NHW & 149 & -. & $\cdots$ & 8.77 & $09-01-62$ & $s$ & u & u & .. & . & - \\
\hline NHW & 150 & $\cdot \cdot$ & $\cdots$ & 24.30 & 09.05 .62 & $\mathrm{~s}$ & $u$ & $u$ & $\cdots$ & .. & . \\
\hline NHW & 151 & .. & .. & 5.0 & $06-16-88$ & $T$ & $u$ & $u$ & .. & .. & . \\
\hline NHW & 152 & -. & -. & 23.23 & $09-01-62$ & s & $w$ & H & . & .. & G \\
\hline NHW & 153 & .. & .. & 19.60 & $08-19-62$ & $\mathrm{~s}$ & w & P & -. & -. & - \\
\hline NHW & 154 & -. & .. & 11.63 & $09 \cdot 01 \cdot 62$ & $\mathrm{~s}$ & w & H & -. & .. & G \\
\hline NHW & 155 & $\cdots$ & $\cdots$ & 10.35 & $07-13-62$ & s & u & $u$ & .. & .. & - \\
\hline
\end{tabular}


Table 1.--Description of selected wells, springs, and test borings--Continued

[-- or -, no data available; 00 in date columns indicates date unknown]

\begin{tabular}{|c|c|c|c|c|c|c|c|c|c|c|c|}
\hline & & & & ALTI TUDE & & & & & & DIAMETER & \\
\hline & & & & OF LAND & METHOD & & METHOD & DEPTH & DEPTH & OF & TYPE \\
\hline $\begin{array}{l}\text { STA } \\
\text { NUM }\end{array}$ & $\begin{array}{l}\text { TION } \\
\text { BER }\end{array}$ & LATITUDE & LONGI TUDE & $\begin{array}{r}\text { SURFACE } \\
\text { (FEET) }\end{array}$ & $\begin{array}{l}\text { ALTITUDE } \\
\text { DETERMINED }\end{array}$ & $\begin{array}{l}\text { DATE OF } \\
\text { CONSTRUCTION }\end{array}$ & $\begin{array}{l}\text { CONST - } \\
\text { RUCTED }\end{array}$ & $\begin{array}{r}\text { DRILLED } \\
\text { (FEET) }\end{array}$ & $\begin{array}{r}\text { OF WELL } \\
\text { (FEET) }\end{array}$ & $\begin{array}{l}\text { CASING } \\
\text { (IN) }\end{array}$ & $\begin{array}{c}\text { OF } \\
\text { FINISH }\end{array}$ \\
\hline NHW & 156 & 411130 & 0713408 & 17 & A & $00-00-54$ & c & 31 & 31 & 6 & $\mathbf{s}$ \\
\hline NHW & 157 & 411127 & 0713413 & 6 & A & $-\cdot$ & D & 6 & 6 & 24 & $W$ \\
\hline NHW & 158 & 411027 & 0713601 & 80 & A & $12-00-54$ & c & 95 & 95 & 6 & $\mathbf{s}$ \\
\hline NHW & 159 & 410936 & 0713337 & 141 & A & $\cdots$ & D & 7 & 7 & $\cdot \cdot$ & w \\
\hline NHW & 160 & 410946 & 0713255 & 35 & A & $\cdots$ & D & 7 & 7 & $\cdots$ & W \\
\hline NHW & 162 & 411025 & 0713353 & 6 & A & $00-00-55$ & D & 9 & 9 & 36 & c \\
\hline NHW & 163 & 411023 & 0713439 & 30 & A & $00 \cdot 00-55$ & D & 13 & 13 & 30 & c \\
\hline NHW & 165 & 411017 & 0713409 & 28 & A & $\cdots$ & D & 9 & 9 & 24 & c \\
\hline NHW & 166 & 411019 & 0713425 & 59 & A & $\cdots$ & $D$ & 20 & 20 & 36 & c \\
\hline NHW & 167 & 410936 & 0713338 & 149 & A & $06-00 \cdot 57$ & c & 150 & 150 & 6 & $s$ \\
\hline NHW & 168 & 411016 & 0713620 & 30 & A & $\cdots$ & D & 17 & 17 & 24 & c \\
\hline NHW & 169 & 411034 & 0713408 & 6 & A & $\cdots$ & D & 9 & 9 & 18 & $W$ \\
\hline NHW & 170 & 411145 & 0713417 & 23 & A & $\cdots$ & c & 45 & 45 & 6 & $s$ \\
\hline NHW & 171 & 411019 & 0713443 & 40 & A & -. & D & 25 & 25 & 36 & w \\
\hline NHW & 172 & 411048 & 0713430 & 16 & A & .. & D & 7 & 7 & $\cdots$ & $w$ \\
\hline NHW & 173 & 411041 & 0713423 & 7 & A & $00-00-56$ & c & 63 & 63 & $\cdots$ & $\cdot$ \\
\hline & & & & & & $\cdots$ & - & & & $\cdots$ & - \\
\hline NHW & 174 & 411046 & 0713412 & 11 & A & $00-00-61$ & D & 12 & 12 & 36 & c \\
\hline NHW & 175 & 411038 & 0713421 & 12 & A & $00-00-44$ & D & 13 & 13 & 24 & $w$ \\
\hline NHW & 176 & 410959 & 0713312 & $1 / 40$ & A & $00-00-32$ & D & 19 & 19 & 24 & c \\
\hline NHW & 177 & 411001 & 0713343 & 90 & A & $00-00-15$ & D & 11 & 11 & $\cdots$ & W \\
\hline NHW & 178 & 410936 & 0713333 & 130 & A & $\cdots$ & $D$ & 6 & 6 & $\cdots$ & $w$ \\
\hline NHW & 179 & 411039 & 0713420 & $1_{35}$ & A & $08-00-55$ & c & 74 & 74 & $\cdots$ & s \\
\hline NHW & 180 & 411025 & 0713359 & 8 & A & .. & D & 9 & 9 & 30 & $w$ \\
\hline NHW & 181 & 411107 & 0713537 & 45 & A & $\cdots$ & D & 9 & 9 & 24 & $w$ \\
\hline NHW & 182 & 411135 & 0713414 & 7 & A & $\cdots$ & D & 7 & 7 & $\cdots$ & $w$ \\
\hline NHW & 183 & 411307 & 0713354 & 23 & A & $12-00-61$ & c & 67 & 67 & $\cdots$ & $\cdot$ \\
\hline NHW & 184 & 410952 & 0713600 & 80 & A & $00-00-57$ & $v$ & 27 & 27 & 2 & $\cdot$ \\
\hline NHW & 185 & 411136 & 0713428 & 10 & A & $\cdots$ & D & 11 & 11 & 24 & $z$ \\
\hline NHW & 186 & 410940 & 0713248 & 75 & A & $00-00-49$ & $D$ & 15 & 15 & 36 & C \\
\hline NHW & 187 & 410942 & 0713246 & $1_{52}$ & A & $00-00-32$ & D & 16 & 16 & 30 & c \\
\hline NHW & 188 & 411024 & 0713345 & 8 & A & -. & D & 8 & 8 & -. & - \\
\hline NHW & 189 & 411016 & 0713421 & 52 & A & $\cdots$ & D & 11 & 11 & 30 & c \\
\hline & & & & & & -. & - & & & 30 & - \\
\hline NHW & 190 & 411133 & 0713412 & 8 & A & $00-00-56$ & D & 10 & 10 & 30 & c \\
\hline NHW & 191 & 411015 & 0713321 & 35 & A & $\cdots$ & $v$ & 22 & 22 & 2 & - \\
\hline NHW & 192 & 411015 & 0713323 & 32 & A & $\cdots$ & D & 11 & 11 & 108 & $z$ \\
\hline NHW & 193 & 411008 & 0713324 & 76 & A & $\cdots$ & D & 43 & 43 & 60 & $w$ \\
\hline NHW & 194 & 411138 & 0713523 & 5 & A & - & $D$ & 10 & 10 & 60 & $w$ \\
\hline NHW & 195 & 411301 & 0713403 & 161 & A & $\cdots$ & D & 10 & 10 & 21 & c \\
\hline
\end{tabular}


Table 1.--Description of selected wells, springs, and test borings--Continued

$$
\text { [- or -, no data available; } 00 \text { in date columns indicates unknown] }
$$

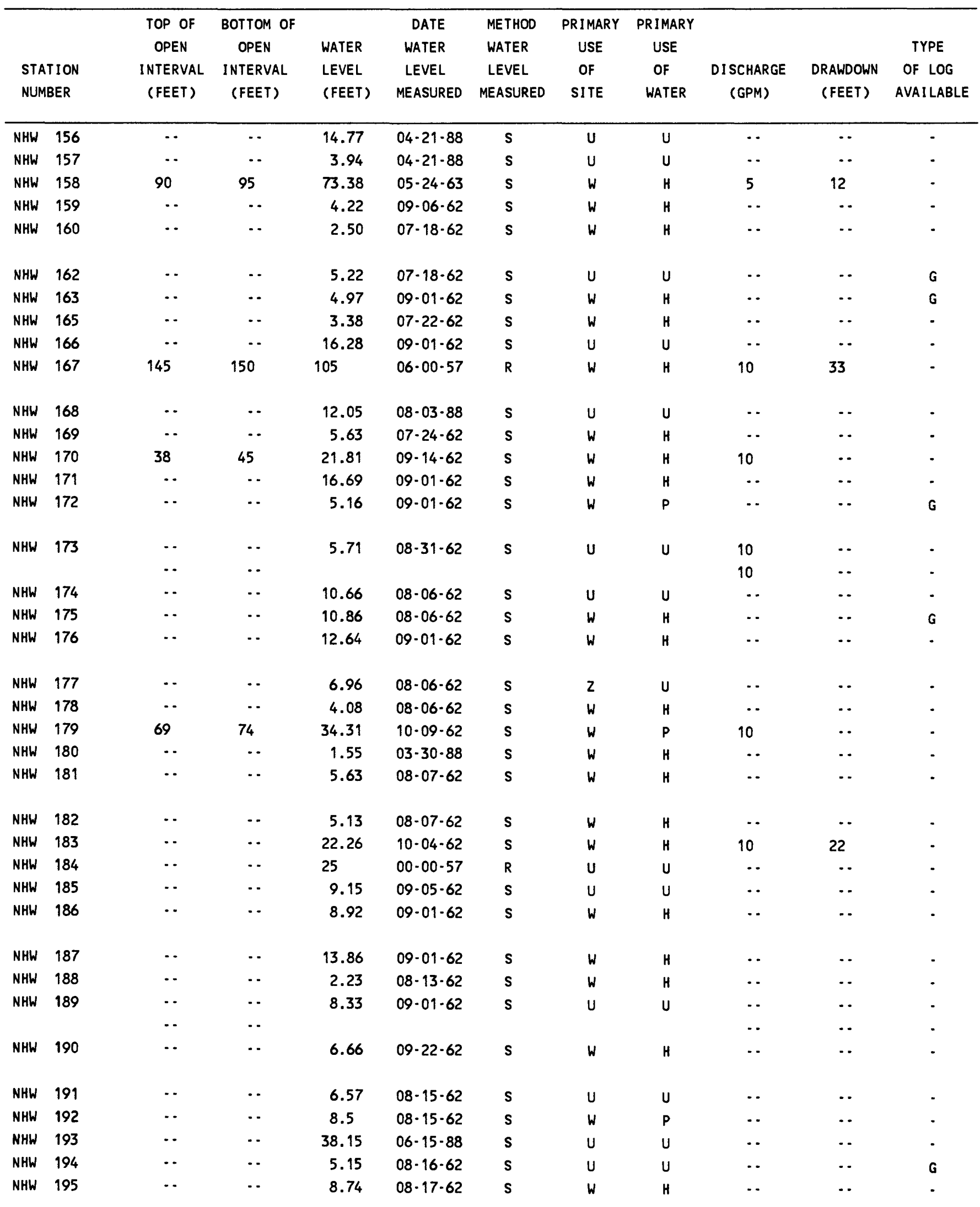


Table 1.--Description of selected wells, springs, and test borings--Continued

$$
\text { [-. or -, no data available; } 00 \text { in date columns indicates date unknown] }
$$

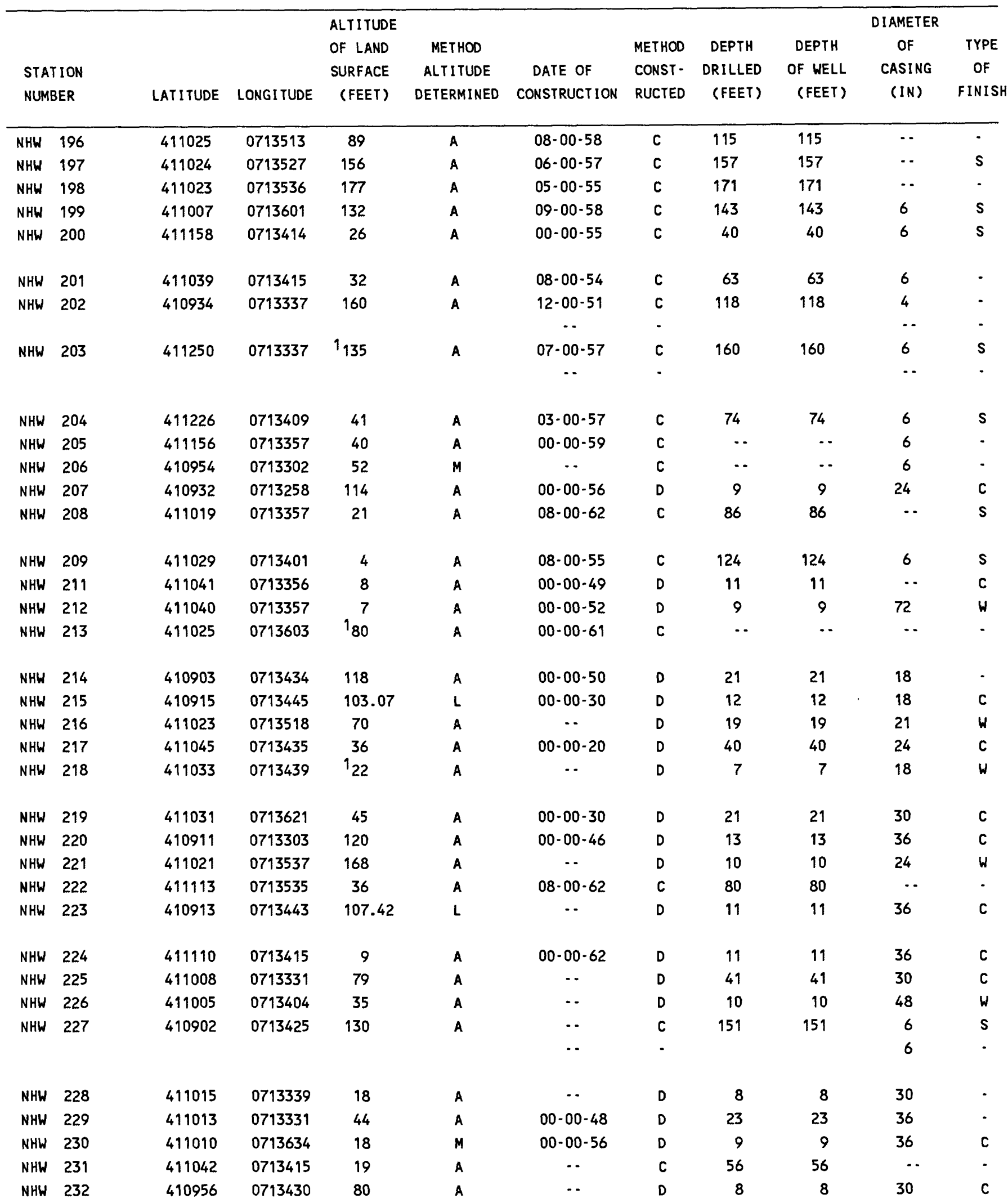


Table 1.--Description of selected wells, springs, and test borings--Continued

[.- or -, no data available; 00 in date columns indicates unknown]

\begin{tabular}{|c|c|c|c|c|c|c|c|c|c|c|c|}
\hline \multicolumn{2}{|c|}{$\begin{array}{l}\text { STATION } \\
\text { NUMBER }\end{array}$} & $\begin{array}{l}\text { TOP OF } \\
\text { OPEN } \\
\text { INTERVAL } \\
\text { (FEET) }\end{array}$ & $\begin{array}{l}\text { BOTTOM OF } \\
\text { OPEN } \\
\text { INTERVAL } \\
\text { (FEET) }\end{array}$ & $\begin{array}{l}\text { WATER } \\
\text { LEVEL } \\
\text { (FEET) }\end{array}$ & $\begin{array}{c}\text { DATE } \\
\text { WATER } \\
\text { LEVEL } \\
\text { MEASURED }\end{array}$ & $\begin{array}{l}\text { METHOD } \\
\text { WATER } \\
\text { LEVEL } \\
\text { MEASURED }\end{array}$ & $\begin{array}{c}\text { PRIMARY } \\
\text { USE } \\
\text { OF } \\
\text { SITE }\end{array}$ & $\begin{array}{c}\text { PRIMARY } \\
\text { USE } \\
\text { OF } \\
\text { WATER }\end{array}$ & $\begin{array}{c}\text { DISCHARGE } \\
\text { (GPM) }\end{array}$ & $\begin{array}{l}\text { DRAWDOWN } \\
\text { (FEET) }\end{array}$ & $\begin{array}{c}\text { TYPE } \\
\text { OF LOG } \\
\text { AVAILABLE }\end{array}$ \\
\hline NHW & 196 & $\cdots$ & $\cdots$ & 80 & $08-00-58$ & $\mathbf{R}$ & $W$ & - & 12 & 25 & - \\
\hline NHW & 197 & 151 & 157 & 126 & $06-00-57$ & $\mathbf{R}$ & $W$ & H & 8 & 26 & - \\
\hline NHW & 198 & $\cdots$ & $\cdots$ & $\cdots$ & $\cdots$ & $\cdot$ & $W$ & $H$ & $\cdots$ & $\cdots$ & $\cdot$ \\
\hline NHW & 199 & 121 & 143 & 100 & $09-00-58$ & $\mathbf{R}$ & w & H & 10 & 30 & $\cdot$ \\
\hline NHW & 200 & 33 & 40 & 26 & $00-00-55$ & $\mathbf{R}$ & W & H & 10 & $\cdots$ & G \\
\hline NHW & 201 & $\cdots$ & $\cdots$ & $\cdots$ & $\cdots$ & - & W & $H$ & $\cdots$ & $\cdots$ & - \\
\hline \multirow[t]{2}{*}{ NHW } & 202 & $\cdots$ & $\cdots$ & 78 & $06-00-63$ & $\mathbf{R}$ & $W$ & $H$ & 6 & 24 & - \\
\hline & & $-\cdot$ & $\cdots$ & & & & & & 5 & $\cdots$ & $\cdot$ \\
\hline \multirow[t]{2}{*}{ NHW } & 203 & 155 & 160 & 135 & $07-00-57$ & $\mathbf{R}$ & $W$ & $H$ & 10 & 20 & $\cdot$ \\
\hline & & $\cdots$ & $\cdots$ & & & & & & 25 & $\cdots$ & $\cdot$ \\
\hline NHW & 204 & 69 & 74 & 58 & $03-00-57$ & $\mathbf{R}$ & $w$ & H & 10 & 10 & - \\
\hline NHW & 205 & $\cdots$ & $\cdots$ & $-\cdot$ & $\cdots$ & - & W & H & $\cdots$ & $\cdots$ & $\cdot$ \\
\hline NHW & 206 & $\cdots$ & $\cdots$ & $\cdots$ & $\cdots$ & $\cdot$ & $w$ & H & $\cdots$ & $\cdots$ & $\cdot$ \\
\hline NHW & 207 & $\cdots$ & $\cdots$ & 2.92 & $06-22 \cdot 88$ & s & $u$ & $u$ & $\cdots$ & $\cdots$ & - \\
\hline NHW & 208 & 76 & 86 & 19.67 & $09-02-62$ & s & $W$ & H & 10 & 40 & $\cdot$ \\
\hline NHW & 209 & 117 & 124 & 3.93 & $05-04-88$ & s & U & U & 15 & 20 & - \\
\hline NHW & 211 & $\cdots$ & $\cdots$ & 7.49 & $08-30-62$ & s & W & H & $\cdots$ & $\cdots$ & - \\
\hline NHW & 212 & $\cdots$ & $\cdots$ & 5.92 & $08-30-62$ & $s$ & W & H & $-\cdot$ & $\cdots$ & $\cdot$ \\
\hline NHW & 213 & $\cdots$ & $\cdots$ & $\cdots$ & - & - & $W$ & $H$ & -. & - & - \\
\hline NHW & 214 & $\cdots$ & $\cdots$ & 4.29 & $08-31-62$ & $s$ & $u$ & $u$ & -. & $\cdots$ & G \\
\hline NHW & 215 & $\cdots$ & $\cdots$ & 8.49 & $09-17-62$ & s & $W$ & H & $\cdots$ & $\cdots$ & - \\
\hline NHW & 216 & $\cdots$ & $\cdots$ & 15.29 & $10-06-62$ & $s$ & $u$ & $u$ & $\cdots$ & $\cdots$ & $\cdot$ \\
\hline NHW & 217 & $\cdots$ & $\cdots$ & 35.30 & $10-10-62$ & s & $U$ & U & $-\cdot$ & $\cdots$ & - \\
\hline NHW & 218 & $\cdots$ & $\cdots$ & 3.61 & $09-01-62$ & s & $W$ & $H$ & -. & $\cdots$ & - \\
\hline NHW & 219 & $\cdots$ & $\cdots$ & 19.40 & $08-05-88$ & s & U & $u$ & $\cdots$ & $\cdots$ & - \\
\hline NHW & 220 & $\cdots$ & -. & 2.84 & $06-15-89$ & $s$ & $W$ & H & $\cdots$ & $\cdots$ & G \\
\hline NHW & 221 & $\cdots$ & $\cdots$ & 2.36 & $09 \cdot 06 \cdot 62$ & $s$ & $u$ & $U$ & $-\cdot$ & $\cdots$ & $\cdot$ \\
\hline NHW & 222 & $\cdots$ & $\cdots$ & 35.44 & $09-07-62$ & s & $W$ & $H$ & 10 & 30 & $\cdot$ \\
\hline NHW & 223 & $\cdots$ & $\cdots$ & 6.57 & $09-08-62$ & s & $u$ & $u$ & $\cdots$ & $\cdots$ & - \\
\hline NHW & 224 & -. & $\cdots$ & 8.97 & $09-16-62$ & s & $W$ & $H$ & 0.6 & 1 & - \\
\hline NHW & 225 & $\cdots$ & $\cdots$ & 37.87 & $09-23-62$ & s & U & U & $\cdots$ & -. & - \\
\hline NHW & 226 & $\cdots$ & $\cdots$ & 6.00 & $09-17-62$ & s & W & H & -- & -- & - \\
\hline \multirow[t]{2}{*}{ NHW } & 227 & 147 & 151 & 124 & $08-31-66$ & $\mathbf{R}$ & W & c & 20 & 7 & D \\
\hline & & 147 & 151 & & & & & & $\cdots$ & $\cdots$ & - \\
\hline NHW & 228 & $\cdots$ & $\cdots$ & 2.99 & $09-17-62$ & $s$ & $u$ & $u$ & $\cdots$ & $\cdots$ & - \\
\hline NHW & 229 & $-\cdot$ & $\cdots$ & 5.43 & $09-17-62$ & s & W & H & - & $\cdots$ & G \\
\hline NHW & 230 & $\cdots$ & $\cdots$ & 4.36 & $09-17-62$ & $s$ & 2 & $U$ & $\cdots$ & $\cdots$ & $\cdot$ \\
\hline NHW & 231 & $\cdots$ & $\cdots$ & 18.89 & $10-10-62$ & $s$ & W & $\mathbf{P}$ & 20 & $\cdots$ & $\cdot$ \\
\hline NHW & 232 & $\cdots$ & $\cdots$ & 2.72 & $09-23-62$ & $\mathrm{~s}$ & $U$ & $u$ & $\cdots$ & $\cdots$ & - \\
\hline
\end{tabular}


Table 1.--Description of selected wells, springs, and test borings--Continued

$$
\text { [-- or -, no data available; } 00 \text { in date columns indicates date unknown] }
$$

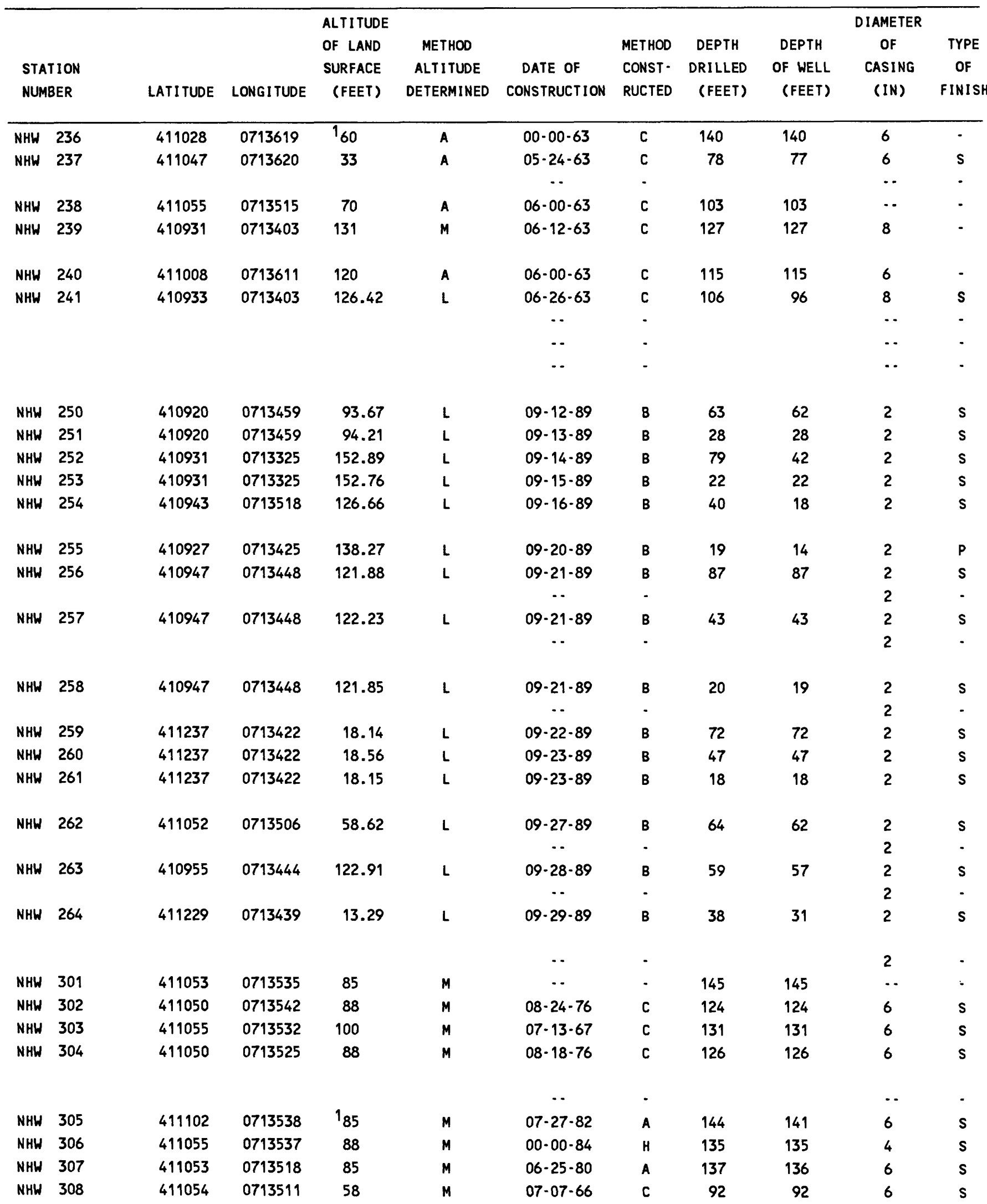


Table 1.--Description of selected wells, springs, and test borings--Continued

$$
\text { [-- or -, no data available; } 00 \text { in date columns indicates unknown] }
$$

\begin{tabular}{|c|c|c|c|c|c|c|c|c|c|c|c|}
\hline $\begin{array}{l}\text { STA } \\
\text { NUM }\end{array}$ & $\begin{array}{l}\text { TION } \\
\text { BER }\end{array}$ & $\begin{array}{l}\text { TOP OF } \\
\text { OPEN } \\
\text { INTERVAL } \\
\text { (FEET) }\end{array}$ & $\begin{array}{l}\text { BOTTOM OF } \\
\text { OPEN } \\
\text { INTERVAL } \\
\text { (FEET) }\end{array}$ & $\begin{array}{l}\text { WATER } \\
\text { LEVEL } \\
\text { (FEET) }\end{array}$ & $\begin{array}{l}\text { DATE } \\
\text { WATER } \\
\text { LEVEL } \\
\text { MEASURED }\end{array}$ & $\begin{array}{l}\text { METHOD } \\
\text { WATER } \\
\text { LEVEL } \\
\text { MEASURED }\end{array}$ & $\begin{array}{l}\text { PRIMARY } \\
\text { USE } \\
\text { OF } \\
\text { SITE }\end{array}$ & $\begin{array}{l}\text { PRIMARY } \\
\text { USE } \\
\text { OF } \\
\text { WATER }\end{array}$ & $\begin{array}{c}\text { DISCHARGE } \\
\text { (GPM) }\end{array}$ & $\begin{array}{l}\text { DRAWDOWN } \\
\text { (FEET) }\end{array}$ & $\begin{array}{c}\text { TYPE } \\
\text { OF LOG } \\
\text { AVAILABLE }\end{array}$ \\
\hline NHW & 236 & $\cdots$ & $\cdots$ & 58.62 & $05 \cdot 24 \cdot 63$ & $\mathbf{s}$ & $W$ & H & $\cdots$ & $\cdots$ & - \\
\hline NHW & 237 & $\begin{array}{c}75 \\
\ldots\end{array}$ & $\begin{array}{c}77 \\
\cdots\end{array}$ & 32.77 & $06-26 \cdot 63$ & $\mathbf{s}$ & $W$ & $H$ & $\begin{array}{l}14 \\
14\end{array}$ & $\begin{array}{l}33 \\
\ldots\end{array}$ & $\begin{array}{l}\text { D } \\
-\end{array}$ \\
\hline NHW & 238 & $\cdots$ & $\cdots$ & 69.9 & $06-25-63$ & s & $W$ & H & 35 & $\cdots$ & D \\
\hline NHW & 239 & $\cdots$ & $\cdots$ & $\cdots$ & $\cdots$ & $\cdot$ & $z$ & $U$ & 4 & $\cdots$ & D \\
\hline NHW & 240 & $\cdots$ & $\cdots$ & 91.79 & $06-26-63$ & s & $W$ & H & $\cdots$ & $-\cdot$ & - \\
\hline NHW & 241 & $\begin{array}{c}86 \\
\ldots \\
\ldots \\
\ldots\end{array}$ & $\begin{array}{c}96 \\
\ldots \\
\ldots \\
\ldots\end{array}$ & 12.37 & $03 \cdot 28 \cdot 88$ & s & $U$ & $U$ & $\begin{array}{l}40 \\
65 \\
90 \\
50\end{array}$ & $\begin{array}{c}20 \\
\ldots \\
20 \\
\ldots\end{array}$ & $\begin{array}{l}\text { D } \\
- \\
- \\
.\end{array}$ \\
\hline NHW & 250 & 59 & 62 & 35.79 & $10-23-89$ & s & 0 & $u$ & $\cdots$ & -- & D \\
\hline NHW & 251 & 24 & 28 & 8.13 & $10-23-89$ & $\mathbf{s}$ & 0 & $U$ & $\cdots$ & $\cdots$ & D \\
\hline NHW & 252 & 39 & 42 & 42.06 & $10-24-89$ & s & 0 & $u$ & $\cdots$ & $\cdots$ & D \\
\hline NHW & 253 & 18 & 22 & 4.98 & $10-24-89$ & s & 0 & $u$ & $\cdots$ & $\cdots$ & D \\
\hline NHW & 254 & 15 & 18 & 4.08 & $10-24-89$ & s & 0 & $U$ & $\cdots$ & $\cdots$ & D \\
\hline NHW & 255 & 13 & 14 & 9.31 & $10-23 \cdot 89$ & s & 0 & $u$ & -. & $\cdots$ & D \\
\hline NHW & 256 & $\begin{array}{c}84 \\
-\cdot\end{array}$ & $\begin{array}{c}87 \\
--\end{array}$ & 81.10 & $10-25-89$ & s & 0 & $u$ & $\cdots$ & $\cdots$ & D \\
\hline NHW & 257 & $\begin{array}{l}38 \\
\ldots\end{array}$ & $\begin{array}{c}43 \\
\cdots\end{array}$ & 25.04 & $10-25-89$ & s & 0 & $u$ & $\cdots$ & $\begin{array}{l}\cdots \\
\cdots\end{array}$ & $\begin{array}{l}\text { D } \\
-\end{array}$ \\
\hline NHW & 258 & $\begin{array}{c}14 \\
-\cdot\end{array}$ & $\begin{array}{l}19 \\
\cdots\end{array}$ & 11.52 & $10-25-89$ & s & 0 & $U$ & $\cdots$ & $\cdots$ & $\begin{array}{l}\text { D } \\
-\end{array}$ \\
\hline NHW & 259 & 67 & 72 & 9.79 & $10-23 \cdot 89$ & s & 0 & $u$ & $\cdots$ & $\cdots$ & D \\
\hline NHW & 260 & 42 & 47 & 9.34 & $10 \cdot 23 \cdot 89$ & s & 0 & $u$ & $\cdots$ & $\cdots$ & D \\
\hline NHW & 261 & 13 & 18 & 8.09 & $10-23-89$ & s & 0 & $U$ & $\cdots$ & $\cdots$ & D \\
\hline NHW & 262 & $\begin{array}{c}57 \\
.-\end{array}$ & $\begin{array}{r}62 \\
\cdots\end{array}$ & 52.38 & $10-24-89$ & $s$ & 0 & $u$ & $\begin{array}{l}-\cdot \\
\cdots\end{array}$ & $\begin{array}{l}\cdots \\
\cdots\end{array}$ & $\begin{array}{l}D \\
-\end{array}$ \\
\hline NHW & 263 & $\begin{array}{r}52 \\
\cdots\end{array}$ & $\begin{array}{c}57 \\
\cdots\end{array}$ & 44.79 & $10-25-89$ & $\mathbf{s}$ & 0 & $u$ & $\begin{array}{l}\cdots \\
\cdots\end{array}$ & $\begin{array}{l}\cdots \\
\cdots\end{array}$ & $\begin{array}{l}\text { D } \\
\cdot\end{array}$ \\
\hline NHW & 264 & $\begin{array}{c}26 \\
\cdots\end{array}$ & $\begin{array}{c}31 \\
\cdots\end{array}$ & 9.25 & $10-25-89$ & s & 0 & $u$ & $\begin{array}{l}\cdots \\
\cdots\end{array}$ & $\begin{array}{l}\cdots \\
\cdots\end{array}$ & D \\
\hline NHW & 301 & $\cdots$ & $\cdots$ & $\cdots$ & $\cdots$ & - & $W$ & $H$ & -. & $\cdots$ & - \\
\hline NHW & 302 & 120 & 124 & 84 & $08-24-76$ & $\mathbf{R}$ & $W$ & $H$ & 50 & $\cdots$ & D \\
\hline NHW & 303 & 127 & 131 & 95 & $07-13-67$ & $\mathbf{R}$ & $W$ & H & 35 & 3 & D \\
\hline NHW & 304 & $\begin{array}{r}123 \\
\ldots\end{array}$ & $\begin{array}{r}126 \\
--\end{array}$ & 88 & $08-18-76$ & $\mathbf{R}$ & $W$ & H & $\begin{array}{l}15 \\
10\end{array}$ & $\begin{array}{c}\cdots \\
10\end{array}$ & $\begin{array}{l}\text { D } \\
-\end{array}$ \\
\hline NHW & 305 & 138 & 141 & 85 & $07-27-82$ & $\mathbf{R}$ & $w$ & H & 80 & 15 & D \\
\hline NHW & 306 & 130 & 135 & -. & $\cdots$ & $\cdot$ & $w$ & H & 60 & $\cdots$ & $\cdot$ \\
\hline NHW & 307 & 134 & 136 & 83 & $06-25-80$ & $\mathbf{R}$ & $w$ & H & 50 & $\cdots$ & $D$ \\
\hline NHW & 308 & 88 & 92 & 55 & $07-07-66$ & $\mathbf{R}$ & $W$ & $H$ & 15 & $\cdots$ & D \\
\hline
\end{tabular}


Table 1.--Description of selected wells, springs, and test borings--Continued

$$
\text { [-- or -, no data available; } 00 \text { in date columns indicates date unknown] }
$$

\begin{tabular}{|c|c|c|c|c|c|c|c|c|c|c|c|}
\hline & & & & ALTITUDE & & & & & & DIAMETER & \\
\hline $\begin{array}{l}\text { STA } \\
\text { NUM }\end{array}$ & $\begin{array}{l}\text { TION } \\
\text { BER }\end{array}$ & LATITUDE & LONGITUDE & $\begin{array}{r}\text { OF LAND } \\
\text { SURFACE } \\
\text { (FEET) }\end{array}$ & $\begin{array}{c}\text { METHOD } \\
\text { ALTITUDE } \\
\text { DETERMINED }\end{array}$ & $\begin{array}{l}\text { DATE OF } \\
\text { CONSTRUCTION }\end{array}$ & $\begin{array}{l}\text { MET HOD } \\
\text { CONST - } \\
\text { RUCTED }\end{array}$ & $\begin{array}{r}\text { DEPTH } \\
\text { DRILLED } \\
\text { (FEET) }\end{array}$ & $\begin{array}{c}\text { DEPTH } \\
\text { OF WELL } \\
\text { (FEET) }\end{array}$ & $\begin{array}{c}\text { OF } \\
\text { CASING } \\
\text { (IN) }\end{array}$ & $\begin{array}{c}\text { TYPE } \\
\text { OF } \\
\text { FINISH }\end{array}$ \\
\hline NHW & 309 & 411054 & 0713514 & 65 & M & $09-16-82$ & A & 93 & 93 & 6 & s \\
\hline NHW & 310 & 411102 & 0713518 & 70 & $M$ & $05-24-84$ & A & 105 & 105 & 6 & $s$ \\
\hline NHW & 312 & 411111 & 0713532 & 70 & M & $07 \cdot 05 \cdot 67$ & C & 121 & 121 & 6 & 0 \\
\hline NHW & 313 & 411116 & 0713534 & 38 & M & $05-30-85$ & A & 103 & 103 & 6 & $s$ \\
\hline NHW & 314 & 411118 & 0713538 & 40 & M & $07-15 \cdot 64$ & c & 71 & 71 & 6 & $s$ \\
\hline & & & & & & - & - & & & $\cdots$ & - \\
\hline NHW & 315 & 411116 & 0713527 & 15 & $M$ & $05-11-82$ & A & 53 & 52 & 6 & s \\
\hline NHW & 316 & 411114 & 0713523 & 5 & $M$ & $06-14-79$ & A & 46 & 32 & 6 & s \\
\hline NHW & 317 & 411112 & 0713517 & $1_{42}$ & $M$ & $05-06-70$ & c & 75 & 75 & 6 & s \\
\hline NHW & 318 & 411059 & 0713501 & 25 & $M$ & $-\cdot$ & - & $-\cdot$ & $\cdots$ & - & - \\
\hline NHW & 319 & 411057 & 0713459 & 24 & $M$ & $00-00-82$ & H & 64 & 64 & -. & $\mathbf{s}$ \\
\hline NHW & 320 & 410919 & 0713303 & 130 & $M$ & $03-02-88$ & H & 245 & 245 & 4 & s \\
\hline NHW & 321 & 411024 & 0713507 & 75 & $M$ & - & H & 112 & 110 & $\cdots$ & s \\
\hline NHW & 322 & 411046 & 0713600 & 98 & $M$ & $09-03-87$ & H & 180 & 180 & 4 & $s$ \\
\hline & & & & & & $\cdots$ & $\cdot$ & & & $\cdots$ & $\cdot$ \\
\hline NHW & 323 & 410948 & 0713454 & 110 & M & $07-11-63$ & C & 119 & 119 & 6 & s \\
\hline NHW & 324 & 410944 & 0713505 & 100 & M & $\cdots$ & H & 185 & 185 & 6 & s \\
\hline NHW & 325 & 410945 & 0713502 & 120 & M & $05-19-82$ & A & 177 & 177 & $\cdots$ & s \\
\hline & & & & & & $\cdots$ & - & & & $\cdots$ & $\cdot$ \\
\hline NHW & 328 & 410953 & 0713511 & 130 & $M$ & $08-06-79$ & A & 220 & 220 & 6 & $s$ \\
\hline NHW & 329 & 410957 & 0713553 & 130 & $M$ & $08 \cdot 12 \cdot 75$ & c & 172 & 172 & 6 & s \\
\hline & & & & & & -. & - & & & $\cdots$ & - \\
\hline NHW & 330 & 410955 & 0713547 & 130 & $M$ & $00-00-72$ & A & 233 & 233 & 6 & $s$ \\
\hline & & & & & & $\cdots$ & - & & & $\cdots$ & $\cdot$ \\
\hline NHW & 331 & 410934 & 0713508 & 167.36 & $L$ & $\cdots$ & - & 203 & 203 & $\cdots$ & - \\
\hline NHW & 332 & 410933 & 0713509 & $1_{160}$ & M & $08-00-87$ & H & 280 & 214 & 4 & $s$ \\
\hline NHW & 333 & 410934 & 0713506 & 165.80 & $L$ & $08-18-87$ & H & 231 & 231 & 4 & s \\
\hline & & & & & & $-\cdot$ & - & & & 4 & $\cdot$ \\
\hline NHW & 334 & 410930 & 0713506 & 149 & M & $08-25-87$ & H & 213 & 213 & 4 & $s$ \\
\hline & & & & & & $\cdots$ & - & & & 4 & - \\
\hline NHW & 335 & 410927 & 0713503 & 144.81 & $L$ & $08-14-87$ & H & 170 & 170 & 4 & s \\
\hline & & & & & & $-\cdot$ & - & & & $\cdots$ & $\cdot$ \\
\hline NHW & 336 & 410944 & 0713641 & 12 & M & $07 \cdot 31-79$ & A & 64 & 64 & 6 & $s$ \\
\hline & & & & & & $\cdots$ & - & & & $\cdots$ & $\cdot$ \\
\hline NHW & 337 & 410928 & 0713438 & 123 & M & $09-18-85$ & A & 123 & 123 & $\cdots$ & $s$ \\
\hline NHW & 338 & 411229 & 0713412 & 63 & $M$ & $00-00-83$ & H & 96 & 96 & 4 & 0 \\
\hline NHW & 339 & 411327 & 0713353 & 12 & $M$ & $00-00-87$ & H & 20 & 17 & 4 & $s$ \\
\hline NHW & 340 & 411250 & 0713335 & $1_{135}$ & $M$ & $06-28-83$ & A & 168 & 162 & 6 & s \\
\hline NHW & 341 & 411250 & 0713352 & 119 & A & $08-04-76$ & c & 170 & 170 & 6 & $\mathbf{s}$ \\
\hline NHW & 342 & 411249 & 0713346 & 125 & $M$ & $09-24-81$ & A & 169 & 169 & 6 & s \\
\hline
\end{tabular}


Table 1.--Description of selected wells, springs, and test borings--Continued [-. or -, no data available; 00 in date columns indicates unknown]

\begin{tabular}{|c|c|c|c|c|c|c|c|c|c|c|c|}
\hline \multicolumn{2}{|c|}{$\begin{array}{l}\text { STATION } \\
\text { NUMBER }\end{array}$} & \multirow{2}{*}{$\begin{array}{c}\text { TOP OF } \\
\text { OPEN } \\
\text { INTERVAL } \\
\text { (FEET) }\end{array}$} & $\begin{array}{l}\text { BOTTOM OF } \\
\text { OPEN } \\
\text { INTERVAL } \\
\text { (FEET) }\end{array}$ & $\begin{array}{l}\text { WATER } \\
\text { LEVEL } \\
\text { (FEET) }\end{array}$ & $\begin{array}{l}\text { DATE } \\
\text { WATER } \\
\text { LEVEL } \\
\text { MEASURED }\end{array}$ & $\begin{array}{l}\text { METHOD } \\
\text { WATER } \\
\text { LEVEL } \\
\text { MEASURED }\end{array}$ & $\begin{array}{c}\text { PRIMARY } \\
\text { USE } \\
\text { OF } \\
\text { SITE }\end{array}$ & $\begin{array}{c}\text { PRIMARY } \\
\text { USE } \\
\text { OF } \\
\text { WATER }\end{array}$ & $\begin{array}{c}\text { DISCHARGE } \\
\text { (GPM) }\end{array}$ & $\begin{array}{l}\text { DRAWDOWN } \\
\text { (FEET) }\end{array}$ & $\begin{array}{c}\text { TYPE } \\
\text { OF LOG } \\
\text { AVAILABLE }\end{array}$ \\
\hline NHW & 309 & & 93 & 62 & $09-16-82$ & $\mathbf{R}$ & $W$ & $H$ & 25 & - & D \\
\hline NHW & 310 & 103 & 105 & 75 & $05-24 \cdot 84$ & $\mathbf{R}$ & $W$ & $H$ & 25 & $\cdots$ & - \\
\hline NHW & 312 & $\cdots$ & $\cdots$ & 70 & $07-05-67$ & $\mathbf{R}$ & $W$ & $H$ & 12 & $-\cdot$ & $\cdot$ \\
\hline NHW & 313 & 101 & 103 & 40 & $05-30-85$ & $\mathbf{R}$ & $W$ & $H$ & 60 & $\cdots$ & D \\
\hline \multirow[t]{2}{*}{ NHW } & 314 & 67 & 71 & 35 & $07-15-64$ & $\mathbf{R}$ & $W$ & $H$ & 13 & 20 & - \\
\hline & & $\cdots$ & $\cdots$ & & & & & & 22 & -. & $\cdot$ \\
\hline NHW & 315 & 49 & 52 & 10 & $05 \cdot 11 \cdot 82$ & $\mathbf{R}$ & $W$ & $H$ & 1 & $\cdots$ & $D$ \\
\hline NHW & 316 & 29 & 32 & 21 & $06-14 \cdot 79$ & $\mathbf{R}$ & $W$ & $H$ & 5 & $\cdots$ & D \\
\hline NHW & 317 & 72 & 75 & 46 & $05-06-70$ & $R$ & $W$ & $H$ & 30 & 14 & D \\
\hline NHW & 318 & $\cdots$ & $\therefore$ & $\cdots$ & $\cdots$ & $\cdot$ & $W$ & c & $\cdots$ & $\cdots$ & $\cdot$ \\
\hline NHW & 319 & $\cdots$ & $\cdots$ & 24 & $00-00-82$ & $\mathbf{R}$ & $w$ & c & -. & $\cdots$ & - \\
\hline NHW & 320 & 240 & 245 & 127.2 & $03-04-88$ & s & $W$ & $H$ & -. & $\cdots$ & D \\
\hline NHW & 321 & 103 & 108 & $\cdots$ & $\cdots$ & $\cdot$ & $w$ & $H$ & 20 & $-\cdot$ & D \\
\hline \multirow[t]{2}{*}{ NHW } & 322 & 170 & 180 & 89.80 & $03-09 \cdot 88$ & $s$ & $U$ & $U$ & 160 & $\cdots$ & $D$ \\
\hline & & $\cdots$ & $\cdots$ & & & & & & $\cdots$ & $\cdot-$ & - \\
\hline NHW & 323 & 115 & 119 & 88 & $07-11-63$ & $\mathbf{R}$ & $W$ & $H$ & 16 & $\cdot-$ & D \\
\hline NHW & 324 & 180 & 185 & 128 & $03-10 \cdot 88$ & $T$ & $W$ & $H$ & $\cdots$ & $\cdots$ & D \\
\hline \multirow[t]{2}{*}{ NHW } & 325 & 174 & 177 & 115 & $05 \cdot 19 \cdot 82$ & $\mathbf{R}$ & $W$ & $H$ & 50 & - & D \\
\hline & & $\cdots$ & $\cdots$ & & & & & & 10 & 20 & $\cdot$ \\
\hline NHW & 328 & 217 & 220 & 124 & $08-06-79$ & $\mathbf{R}$ & $w$ & $H$ & 70 & $\cdots$ & D \\
\hline \multirow[t]{2}{*}{ NHW } & 329 & 168 & 172 & 102 & $08 \cdot 12 \cdot 75$ & $\mathbf{R}$ & $w$ & $H$ & 7 & 48 & D \\
\hline & & $\cdots$ & $\cdots$ & & & & & & 5 & 28 & $\cdot$ \\
\hline \multirow[t]{2}{*}{ NHW } & 330 & 230 & 233 & 130 & $00 \cdot 00 \cdot 72$ & $\mathbf{R}$ & $w$ & $H$ & 12 & $\cdots$ & D \\
\hline & & $\cdots$ & $\cdots$ & & & & & & 60 & $\cdots$ & $\cdot$ \\
\hline NHW & 331 & $\cdots$ & $\cdots$ & 160.1 & $03-10-88$ & $T$ & $W$ & H & $\cdots$ & $\cdots$ & - \\
\hline NHW & 332 & 209 & 214 & 155 & $08-14-87$ & $\mathbf{R}$ & $W$ & $H$ & 20 & $\cdots$ & D \\
\hline \multirow[t]{2}{*}{ NHW } & 333 & 170 & 175 & 160.5 & $03 \cdot 10 \cdot 88$ & $T$ & $\mathrm{U}$ & $U$ & 2 & $\cdots$ & $\cdot$ \\
\hline & & $\cdots$ & $\cdots$ & & & & & & $-\cdot$ & $\cdots$ & D \\
\hline \multirow[t]{2}{*}{ NHW } & 334 & 192 & 197 & 147 & $08-28-87$ & s & $w$ & $H$ & 5 & $\cdots$ & D \\
\hline & & $\cdots$ & $\cdots$ & & & & & & $\cdots$ & $\cdots$ & $\cdot$ \\
\hline \multirow[t]{2}{*}{ NHW } & 335 & 160 & 170 & 140.4 & $03-11-88$ & $T$ & $w$ & $H$ & 8 & -. & $\cdot$ \\
\hline & & $\cdots$ & $\cdots$ & & & & & & $\cdots$ & -. & D \\
\hline \multirow[t]{2}{*}{ NHW } & 336 & 61 & 64 & 22 & $07-31-79$ & $\mathbf{R}$ & $W$ & $H$ & 30 & 42 & $D$ \\
\hline & & $\cdots$ & $\cdots$ & & & & & & 7 & 6 & $\cdot$ \\
\hline NHW & 337 & 120 & 123 & 110 & $09-18 \cdot 85$ & $\mathbf{R}$ & $W$ & $H$ & 10 & $\cdots$ & $D$ \\
\hline NHW & 338 & - & $\cdots$ & 61.10 & $03 \cdot 11 \cdot 88$ & $\mathbf{s}$ & $w$ & $H$ & $\cdots$ & $\cdots$ & - \\
\hline NHW & 339 & 12 & 17 & 6.17 & $03 \cdot 11 \cdot 88$ & s & $w$ & $H$ & $\cdots$ & $\cdots$ & $D$ \\
\hline NHW & 340 & 159 & 162 & 135 & $06-28-83$ & $\mathbf{R}$ & $w$ & $H$ & 28 & $-\cdot$ & D \\
\hline NHW & 341 & 167 & 170 & 118 & $08-04-76$ & $R$ & $W$ & $H$ & 5 & $\cdots$ & D \\
\hline NHW & 342 & 166 & 169 & 127 & $09 \cdot 24-81$ & $\mathbf{R}$ & $W$ & $H$ & 20 & $\cdots$ & D \\
\hline
\end{tabular}


Table 1.--Description of selected wells, springs, and test borings--Continued

$$
\text { [-- or -, no data available; } 00 \text { in date columns indicates date unknown] }
$$

\begin{tabular}{|c|c|c|c|c|c|c|c|c|c|c|c|}
\hline & & & & ALT I TUDE & & & & & & DIAMETER & \\
\hline & & & & OF LAND & METHOD & & METHOD & DEPTH & DEPTH & & TYPE \\
\hline STA & TION & & & SURFACE & ALTITUDE & DATE OF & CONST - & DRILLED & OF WELL & CASING & OF \\
\hline NUM & BER & LATI TUDE & LONGITUDE & (FEET) & DETERMINED & CONSTRUCTION & RUCTED & (FEET) & (FEET) & $(I N)$ & FINISH \\
\hline NHW & 343 & 411234 & 0713354 & 69 & M & $10-06-81$ & $A$ & 139 & 139 & 6 & s \\
\hline NHW & 344 & 411315 & 0713354 & 130 & M & $05-29-80$ & A & 85 & 85 & 6 & s \\
\hline NHW & 345 & 411305 & 0713345 & 65 & M & $06-17-66$ & C & 97 & 97 & 6 & $\mathbf{s}$ \\
\hline NHW & 346 & 411300 & 0713409 & 75 & M & $08-30-78$ & C & 97 & 97 & 6 & s \\
\hline NHW & 347 & 411301 & 0713355 & 60 & M & $07-14-81$ & A & 154 & 107 & 6 & S \\
\hline & & & & & & $\cdots$ & - & & & $\cdots$ & - \\
\hline NHW & 348 & 411231 & 0713415 & 63 & $M$ & $06-21-83$ & A & 114 & 114 & 6 & S \\
\hline NHW & 349 & 411229 & 0713418 & 160 & M & $06-12 \cdot 79$ & A & 105 & 105 & 6 & S \\
\hline NHW & 350 & 411229 & 0713422 & 40 & M & $09-12-73$ & C & 112 & 98 & 6 & $S$ \\
\hline NHW & 351 & 411229 & 0713432 & 35 & $M$ & $03-00-83$ & H & 65 & 65 & 4 & $s$ \\
\hline NHW & 352 & 411235 & 0713332 & 75 & $M$ & $08-02-63$ & C & 108 & 108 & 6 & $s$ \\
\hline NHW & 353 & 411216 & 0713415 & 48 & $M$ & $05-29 \cdot 80$ & A & 84 & 84 & 6 & $s$ \\
\hline NHW & 354 & 411214 & 0713351 & 25 & M & $05-11 \cdot 72$ & C & 60 & 60 & 6 & s \\
\hline NHW & 355 & 411208 & 0713350 & 32 & $M$ & $08 \cdot 20 \cdot 75$ & c & 68 & 68 & 6 & $\mathbf{s}$ \\
\hline NHW & 356 & 411206 & 0713416 & 25 & $M$ & $07-07-87$ & $H$ & 65 & 65 & 4 & $s$ \\
\hline NHW & 357 & 411135 & 0713404 & 25 & $M$ & $\cdots$ & H & 75 & 75 & 6 & $s$ \\
\hline NHW & 358 & 411214 & 0713404 & 35 & $M$ & $04-05 \cdot 87$ & H & 55 & 55 & 4 & $s$ \\
\hline NHW & 359 & 411301 & 0713404 & 62 & $M$ & $00-00-86$ & H & 110 & 110 & $\cdots$ & - \\
\hline NHW & 360 & 411219 & 0713421 & 45 & $M$ & $09-24-75$ & C & 74 & 74 & 6 & s \\
\hline NHW & 361 & 411217 & 0713411 & 40 & $M$ & $07-27-71$ & c & 64 & 64 & 6 & s \\
\hline NHW & 362 & 411200 & 0713408 & 32 & M & $07-05-72$ & $c$ & 73 & 73 & 6 & $s$ \\
\hline NHW & 363 & 411158 & 0713359 & 32 & $M$ & $05-13-76$ & C & 63 & 62 & 6 & $s$ \\
\hline NHW & 364 & 411156 & 0713357 & 45 & M & $06-13-79$ & A & 65 & 64 & 6 & s \\
\hline NHW & 365 & 411151 & 0713415 & 30 & M & $08-11 \cdot 87$ & A & 64 & 64 & 6 & $s$ \\
\hline NHW & 366 & 411143 & 0713423 & 19 & M & $05-21-69$ & C & 66 & 48 & 6 & s \\
\hline NHW & 367 & 411143 & 0713418 & 20 & $M$ & $04-24-68$ & $c$ & 53 & 53 & $\cdots$ & s \\
\hline NHW & 369 & 411036 & 0713422 & 12 & $M$ & $07-19-66$ & C & 107 & 107 & 6 & s \\
\hline NHW & 370 & 411037 & 0713418 & 37.81 & $L$ & $10-15-69$ & C & 95 & 95 & 6 & s \\
\hline NHW & 371 & 411128 & 0713412 & 10 & $M$ & $08 \cdot 00 \cdot 87$ & $H$ & 23 & 23 & 6 & $\cdot$ \\
\hline NHW & 372 & 411042 & 0713407 & 7 & $M$ & $05 \cdot 12 \cdot 78$ & $\mathrm{C}$ & 58 & 58 & 6 & $s$ \\
\hline NHW & 373 & 411027 & 0713437 & $1_{30}$ & $M$ & $06-01-77$ & C & 63 & 63 & 6 & s \\
\hline NHW & 374 & 411033 & 0713441 & 50 & $M$ & $09-30-65$ & C & 109 & 109 & 6 & $s$ \\
\hline NHW & 376 & 411045 & 0713435 & 42 & $M$ & $10-18 \cdot 73$ & C & 161 & 160 & $\cdots$ & s \\
\hline NHW & 377 & 411043 & 0713441 & 33 & $M$ & $09 \cdot 02-82$ & A & 60 & 56 & 6 & $s$ \\
\hline NHW & 378 & 411051 & 0713444 & 11 & M & $06-21-76$ & c & 60 & 56 & 6 & s \\
\hline NHW & 379 & 411208 & 0713409 & 31 & $M$ & $05-17-66$ & C & 83 & 83 & 6 & s \\
\hline NHW & 380 & 411025 & 0713400 & 10 & $M$ & $10-18-83$ & A & 131 & 131 & 6 & s \\
\hline NHW & 381 & 411025 & 0713358 & 10 & M & $06-09-82$ & A & 123 & 123 & 6 & $\mathbf{S}$ \\
\hline NHW & 382 & 411128 & 0713424 & 10 & M & $05-12-67$ & C & 49 & 22 & 6 & $S$ \\
\hline NHW & 383 & 411016 & 0713408 & 39.79 & L & $09-29 \cdot 77$ & C & 123 & 123 & 6 & s \\
\hline
\end{tabular}


Table 1. -Description of selected wells, springs, and test borings--Continued

$$
\text { [-. or -, no data available; } 00 \text { in date columns indicates unknown] }
$$

\begin{tabular}{|c|c|c|c|c|c|c|c|c|c|c|c|}
\hline \multicolumn{2}{|c|}{$\begin{array}{l}\text { STATION } \\
\text { NUMBER }\end{array}$} & \multirow{2}{*}{$\begin{array}{l}\text { TOP OF } \\
\text { OPEN } \\
\text { INTERVAL } \\
\text { (FEET) } \\
136\end{array}$} & \multirow{2}{*}{$\begin{array}{c}\text { BOTTOM OF } \\
\text { OPEN } \\
\text { INTERVAL } \\
\text { (FEET) } \\
139\end{array}$} & \multirow{2}{*}{$\begin{array}{l}\text { WATER } \\
\text { LEVEL } \\
\text { (FEET) } \\
69\end{array}$} & \multirow{2}{*}{$\begin{array}{c}\text { DATE } \\
\text { WATER } \\
\text { LEVEL } \\
\text { MEASURED } \\
10-06-81\end{array}$} & \multirow{2}{*}{$\begin{array}{l}\text { METHOD } \\
\text { WATER } \\
\text { LEVEL } \\
\text { MEASURED } \\
R\end{array}$} & \multirow{2}{*}{$\begin{array}{c}\text { PRIMARY } \\
\text { USE } \\
\text { OF } \\
\text { SITE } \\
W\end{array}$} & \multirow{2}{*}{$\begin{array}{c}\text { PRIMARY } \\
\text { USE } \\
\text { OF } \\
\text { WATER }\end{array}$} & \multirow{2}{*}{$\begin{array}{c}\text { DISCHARGE } \\
\text { (GPM) } \\
100\end{array}$} & \multirow{2}{*}{$\begin{array}{c}\begin{array}{c}\text { ORAWDOWN } \\
\text { (FEET) }\end{array} \\
\cdots\end{array}$} & \multirow{2}{*}{$\begin{array}{c}\text { TYPE } \\
\text { OF LOG } \\
\text { AVAILABLE } \\
\text { D }\end{array}$} \\
\hline NHW & 343 & & & & & & & & & & \\
\hline NHW & 344 & 82 & 85 & 28 & $05-29-80$ & R & $W$ & H & 40 & $\cdots$ & D \\
\hline NHW & 345 & 93 & 97 & 65 & $06-17-66$ & $R$ & $W$ & H & 30 & 0 & D \\
\hline NHW & 346 & 94 & 97 & 74 & $08 \cdot 30-78$ & $\mathbf{R}$ & $W$ & H & 9 & 23 & D \\
\hline NHW & 347 & 104 & 107 & 54 & $07 \cdot 14 \cdot 81$ & $R$ & $W$ & H & 50 & $\cdots$ & D \\
\hline & & $\cdots$ & $\cdots$ & & & & & & 10 & 6 & $\cdot$ \\
\hline NHW & 348 & 111 & 114 & 60 & $06 \cdot 21 \cdot 83$ & $\mathbf{R}$ & $W$ & H & 8 & 30 & D \\
\hline NHW & 349 & 102 & 105 & 58 & $06-12-79$ & $\mathbf{R}$ & $W$ & H & 60 & $\cdots$ & D \\
\hline NHW & 350 & 95 & 98 & 52 & $09 \cdot 12 \cdot 73$ & $\mathbf{R}$ & $W$ & H & 15 & 26 & D \\
\hline NHW & 351 & 62 & 65 & $\cdots$ & $\cdots$ & $\cdot$ & $W$ & $H$ & $\cdots$ & $\cdots$ & $\cdot$ \\
\hline NHW & 352 & 104 & 108 & 68 & $08-02 \cdot 63$ & $\mathbf{R}$ & $W$ & H & 25 & $\because$ & $D$ \\
\hline NHW & 353 & 81 & 84 & 47 & $05 \cdot 29 \cdot 80$ & $R$ & $W$ & H & 10 & 8 & 0 \\
\hline NHW & 354 & 57 & 60 & 26 & $05 \cdot 11 \cdot 72$ & $R$ & $W$ & H & 25 & $\cdots$ & 0 \\
\hline NHW & 355 & 65 & 68 & 32 & $08-20 \cdot 75$ & $\mathbf{R}$ & $W$ & H & 25 & $\cdots$ & 0 \\
\hline NHW & 356 & $\cdots$ & $\cdots$ & 26 & $07 \cdot 07 \cdot 87$ & $\mathbf{R}$ & $W$ & H & 40 & $\cdots$ & 0 \\
\hline NHW & 357 & $\cdots$ & $\cdots$ & $\cdots$ & $\cdots$ & $\cdot$ & $W$ & H & 70 & $\cdots$ & - \\
\hline NHW & 358 & $\cdots$ & $\cdots$ & $\cdots$ & $\cdots$ & $\cdot$ & $W$ & H & 40 & $\cdots$ & 0 \\
\hline NHW & 359 & $\cdots$ & $\cdots$ & $\cdots$ & $\cdots$ & $\cdot$ & $W$ & H & 6 & $\cdots$ & $\cdot$ \\
\hline NHW & 360 & 70 & 74 & 47 & $09-24 \cdot 75$ & R & $w$ & $H$ & 50 & 0 & $D$ \\
\hline NHW & 361 & 61 & 64 & 40 & $07-27-71$ & $R$ & $W$ & H & 35 & 0 & D \\
\hline NHW & 362 & 70 & 73 & 21 & $07-05-72$ & $R$ & W & H & 20 & $\cdots$ & $D$ \\
\hline NHW & 363 & 59 & 62 & 34 & $05 \cdot 13 \cdot 76$ & $R$ & $W$ & $H$ & 45 & $\because$ & $D$ \\
\hline NHW & 364 & 61 & 64 & 39 & $06 \cdot 13 \cdot 79$ & R & $W$ & H & 55 & 25 & $D$ \\
\hline NHW & 365 & 61 & 64 & $\cdots$ & $\cdots$ & - & $W$ & H & 70 & $\cdots$ & 0 \\
\hline NHW & 366 & 44 & 48 & 16 & $05-21-69$ & $R$ & W & H & 25 & $\cdots$ & D \\
\hline NHW & 367 & 49 & 53 & 19 & $04-24-68$ & $\mathbf{R}$ & $w$ & H & 35 & 21 & $D$ \\
\hline NHW & 369 & 103 & 107 & 30 & $07-19 \cdot 66$ & $\mathbf{R}$ & $w$ & H & 10 & $\cdot \cdot$ & 0 \\
\hline NHW & 370 & 92 & 95 & 40 & $10 \cdot 15 \cdot 69$ & $\mathbf{R}$ & $W$ & $F$ & 25 & 36 & 0 \\
\hline NHW & 371 & $\because$ & $\cdots$ & 9.9 & $04-28 \cdot 88$ & $T$ & $z$ & u & $\cdots$ & $\cdots$ & 0 \\
\hline NHW & 372 & 54 & 58 & 4 & $05-12-78$ & $R$ & $W$ & H & 15 & 26 & 0 \\
\hline NHW & 373 & 60 & 63 & 20 & $06 \cdot 01 \cdot 77$ & $R$ & $W$ & H & 15 & $\cdots$ & 0 \\
\hline NHW & 374 & 105 & 109 & 42 & $09 \cdot 30 \cdot 65$ & $\mathbf{R}$ & $W$ & H & 15 & $\cdots$ & $D$ \\
\hline NHW & 376 & 156 & 160 & 33 & $10-18 \cdot 73$ & $\mathbf{R}$ & $W$ & H & 14 & 37 & $D$ \\
\hline NHW & 377 & 53 & 56 & 20 & 09.02 .82 & $\mathbf{R}$ & $W$ & $H$ & 30 & $\cdots$ & D \\
\hline NHW & 378 & 53 & 56 & 12 & $06 \cdot 21 \cdot 76$ & $\mathbf{R}$ & $W$ & C & 18 & $\cdots$ & D \\
\hline NHW & 379 & 79 & 83 & 40 & $05-17-66$ & $\mathbf{R}$ & 2 & $U$ & 35 & 25 & D \\
\hline NHW & 380 & 128 & 131 & 4 & $10-18-83$ & $R$ & $w$ & H & 100 & $\cdots$ & 0 \\
\hline NHW & 381 & 120 & 123 & 2 & $06-09 \cdot 82$ & $\mathbf{R}$ & $W$ & H & 50 & $\cdots$ & $D$ \\
\hline NHW & 382 & 19 & 22 & 10.53 & $04 \cdot 21-88$ & s & W & H & 4 & $\cdots$ & D \\
\hline NHW & 383 & 120 & 123 & 38 & $09-29 \cdot 77$ & $\mathbf{R}$ & $W$ & H & 3 & $\because$ & $D$ \\
\hline
\end{tabular}


Table 1.-Description of selected wells, springs, and test borings--Continued

$$
\text { [-- or -, no data available; } 00 \text { in date columns indicates date unknown] }
$$

\begin{tabular}{|c|c|c|c|c|c|c|c|c|c|c|c|}
\hline & & & & ALTITUDE & & & & & & DIAMETER & \\
\hline $\begin{array}{l}\text { STA } \\
\text { NUM }\end{array}$ & $\begin{array}{l}\text { TION } \\
\text { BER }\end{array}$ & LATITUDE & LONGITUDE & $\begin{array}{r}\text { OF LAND } \\
\text { SURFACE } \\
\text { (FEET) }\end{array}$ & $\begin{array}{c}\text { METHOD } \\
\text { ALTITUDE } \\
\text { DETERMINED }\end{array}$ & $\begin{array}{l}\text { DATE OF } \\
\text { CONSTRUCTION }\end{array}$ & $\begin{array}{l}\text { METHOD } \\
\text { CONST - } \\
\text { RUCTED }\end{array}$ & $\begin{array}{l}\text { DEPTH } \\
\text { DRILLED } \\
\text { (FEET) }\end{array}$ & $\begin{array}{l}\text { DEPTH } \\
\text { OF WELL } \\
\text { (FEET) }\end{array}$ & $\begin{array}{c}\text { OF } \\
\text { CASING } \\
\text { (IN) }\end{array}$ & $\begin{array}{c}\text { TYPE } \\
\text { OF } \\
\text { FINISH }\end{array}$ \\
\hline NHW & 384 & 411022 & 0713333 & 12 & $M$ & $06-17-81$ & A & 86 & 86 & 6 & $\mathbf{S}$ \\
\hline NHW & 385 & 411021 & 0713331 & 15 & M & $05 \cdot 13 \cdot 83$ & A & 70 & 70 & 6 & $\mathbf{s}$ \\
\hline NHW & 386 & 410948 & 0713305 & 60 & $M$ & $07-25-85$ & A & 119 & 119 & $\cdots$ & s \\
\hline NHW & 387 & 411209 & 0713409 & 31 & M & $06-23 \cdot 70$ & c & 83 & 82 & 6 & $\mathbf{s}$ \\
\hline NHW & 388 & 411231 & 0713405 & 57 & M & $03-00-88$ & H & 80 & 80 & 4 & $\mathbf{s}$ \\
\hline NHW & 389 & 411231 & 0713420 & 55 & M & $\begin{array}{c}00 \cdot 00 \cdot 87 \\
\ldots\end{array}$ & $\begin{array}{l}H \\
-\end{array}$ & 100 & 72 & $\begin{array}{l}4 \\
--\end{array}$ & $\begin{array}{l}\text { S } \\
-\end{array}$ \\
\hline NHW & 390 & 411219 & 0713433 & 23 & M & $12-00 \cdot 85$ & $H$ & 68 & 68 & 4 & s \\
\hline NHW & 391 & 411043 & 0713415 & 20 & M & $05 \cdot 05 \cdot 76$ & C & 64 & 64 & 6 & $\mathbf{s}$ \\
\hline NHW & 392 & 411139 & 0713418 & 9 & M & $05-17-72$ & C & 40 & 38 & 6 & $\mathbf{S}$ \\
\hline NHW & 393 & 411139 & 0713417 & 5 & M & $\cdots$ & $D$ & 8 & 8 & -- & $\cdot$ \\
\hline NHW & 394 & 411313 & 0713357 & 15 & M & $\cdots$ & D & 8 & 8 & 30 & 0 \\
\hline NHW & 395 & 411313 & 0713357 & 15 & $M$ & $10-10 \cdot 66$ & C & 42 & 42 & 6 & $\mathrm{~s}$ \\
\hline NHW & 396 & 411308 & 0713358 & 20 & $M$ & $06-29-79$ & 2 & 128 & 128 & 6 & $\mathbf{S}$ \\
\hline NHW & 397 & 411041 & 0713415 & 30 & M & $08-01-68$ & C & 60 & 60 & 6 & s \\
\hline NHW & 399 & 411027 & 0713343 & 12 & M & $05-11-83$ & A & 91 & 90 & 6 & s \\
\hline NHW & 400 & 411008 & 0713409 & 55 & $M$ & $06-06-72$ & C & 137 & 137 & 6 & $\mathbf{s}$ \\
\hline NHW & 401 & 410958 & 0713403 & 46.08 & L & $06-25-81$ & C & 112 & 111 & 6 & s \\
\hline NHW & 402 & 410956 & 0713400 & ${ }^{1} 60.70$ & L & $\begin{array}{c}05-19-76 \\
\cdots\end{array}$ & $\begin{array}{l}\text { C } \\
-\end{array}$ & 145 & 144 & $\begin{array}{l}6 \\
\cdots\end{array}$ & $\begin{array}{l}\text { S } \\
-\end{array}$ \\
\hline NHW & 403 & 411010 & 0713403 & 48 & M & $10-31 \cdot 69$ & C & 157 & 155 & 6 & s \\
\hline NHW & 404 & 410903 & 0713405 & 128 & M & $10-06-67$ & C & 134 & 134 & 6 & s \\
\hline NHW & 405 & 410941 & 0713619 & 70 & M & $08-09-83$ & A & 144 & 144 & 6 & s \\
\hline NHW & 406 & 411023 & 0713544 & 159 & M & $10-24-72$ & c & 178 & 178 & 6 & s \\
\hline NHW & 407 & 411234 & 0713333 & 65 & M & $08-17-77$ & C & 90 & 90 & $\cdots$ & $\cdot$ \\
\hline NHW & 408 & 411025 & 0713406 & 5 & M & $00-00-83$ & D & 6 & 6 & 48 & c \\
\hline NHW & 409 & 411023 & 0713403 & 5 & $M$ & $\cdots$ & D & $\cdots$ & $\cdots$ & $\cdots$ & $\cdot$ \\
\hline NHW & 410 & 411026 & 0713433 & 35 & $M$ & $07-20-73$ & C & 84 & 82 & 6 & $\mathbf{S}$ \\
\hline NHW & 411 & 411028 & 0713430 & 30 & M & $09 \cdot 10-74$ & C & 71 & 70 & 6 & $\mathbf{S}$ \\
\hline NHW & 412 & 411026 & 0713439 & 53 & M & $08-06-64$ & C & 90 & 90 & $\cdots$ & $\mathbf{s}$ \\
\hline NHW & 413 & 411020 & 0713441 & 40 & M & $00-00-64$ & C & 75 & 75 & $\cdots$ & $\cdot$ \\
\hline NHW & 414 & 411014 & 0713429 & 50 & M & $00-00-83$ & B & 85 & 85 & $\cdots$ & s \\
\hline NHW & 415 & 411324 & 0713343 & 45 & $M$ & $08-25-78$ & C & 68 & 68 & 6 & s \\
\hline NHW & 416 & 411011 & 0713634 & 28 & A & $\begin{array}{c}07-09-74 \\
\ldots\end{array}$ & C & 45 & 44 & $\begin{array}{l}6 \\
\cdots\end{array}$ & $\begin{array}{l}\text { S } \\
-\end{array}$ \\
\hline NHW & 417 & 410933 & 0713408 & 128.46 & $L$ & $\begin{array}{c}05-21-81 \\
\ldots\end{array}$ & A & 247 & 247 & $\begin{array}{l}6 \\
\cdots\end{array}$ & S \\
\hline NHW & 418 & 411049 & 0713453 & 35 & M & $\cdots$ & D & 7 & 7 & 24 & $W$ \\
\hline NHW & 419 & 411036 & 0713418 & 35 & $M$ & $06-08-88$ & $H$ & 53 & 52 & 4 & $\mathbf{S}$ \\
\hline NHW & 421 & 410940 & 0713251 & 85 & $M$ & $12-00-87$ & $H$ & 211 & 211 & 4 & $\mathbf{S}$ \\
\hline
\end{tabular}


Table 1.--Description of selected wells, springs, and test borings--Continued

[-- or -, no data available; 00 in date columns indicates unknown]

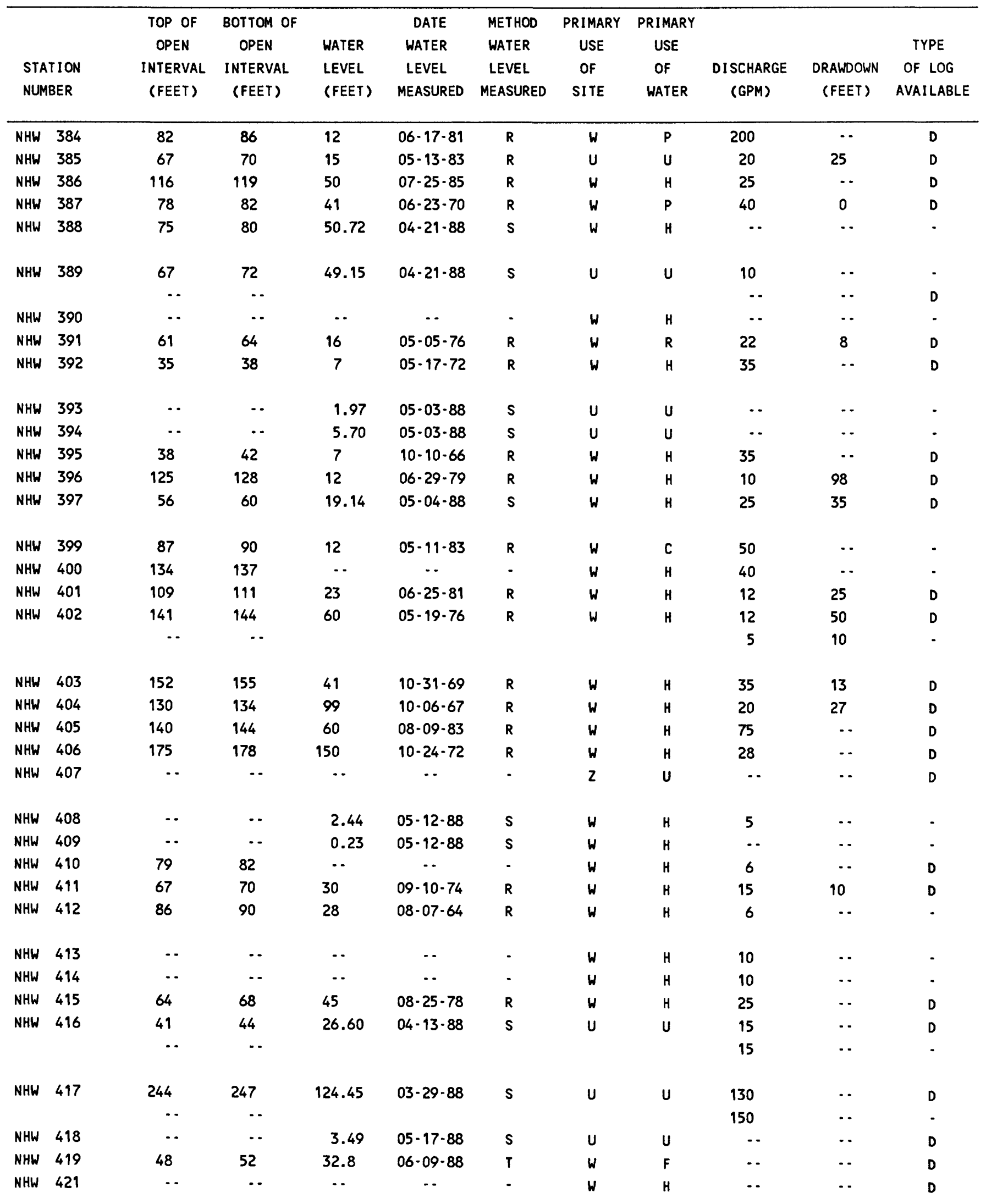


Table 1.--Description of selected wells, springs, and test borings--Continued

$$
\text { [-- or -, no data available; } 00 \text { in date columns indicates date unknown] }
$$

\begin{tabular}{|c|c|c|c|c|c|c|c|c|c|c|c|}
\hline & & & & ALTITUDE & & & & & & DIAMETER & \\
\hline & $\begin{array}{l}\text { IION } \\
\text { SER }\end{array}$ & LATITUDE & LONGITUDE & $\begin{array}{l}\text { OF LAND } \\
\text { SURFACE } \\
\text { (FEET) }\end{array}$ & $\begin{array}{c}\text { METHOD } \\
\text { ALTI IUDEE } \\
\text { DETERMINED }\end{array}$ & $\begin{array}{l}\text { DATE OF } \\
\text { CONSTRUCTION }\end{array}$ & $\begin{array}{l}\text { METHOD } \\
\text { CONST - } \\
\text { RUCTED }\end{array}$ & $\begin{array}{c}\text { DEPTH } \\
\text { DRILLED } \\
\text { (FEET) }\end{array}$ & $\begin{array}{c}\text { DEPTH } \\
\text { OF WELL } \\
\text { (FEET) }\end{array}$ & $\begin{array}{c}\text { OF } \\
\text { CASING } \\
\text { (IN) }\end{array}$ & $\begin{array}{c}\text { TYPE } \\
\text { OF } \\
\text { FINISH }\end{array}$ \\
\hline NHW & 422 & 411037 & 0713457 & 70 & M & $06-01-88$ & $\mathrm{H}$ & 239 & 239 & 4 & s \\
\hline NHW & 424 & 410936 & 0713357 & 121.93 & L & $00.00 \cdot 65$ & c & 130 & 130 & -. & s \\
\hline NHW & 425 & 410929 & 0713404 & 136.85 & L & $08 \cdot 06 \cdot 65$ & c & 246 & 246 & 8 & s \\
\hline & & & & & & . & - & & & 6 & - \\
\hline & & & & & & .- & $\cdot$ & & & $\cdot \cdot$ & $\cdot$ \\
\hline NHW & 426 & 411027 & 0713419 & 15 & M & $11 \cdot 11-67$ & c & 138 & 138 & $\cdots$ & s \\
\hline NHW & 427 & 411059 & 0713458 & 20 & M & . & D & .. & .. & 30 & c \\
\hline NHW & 428 & 411059 & 0713500 & 26 & M & $05-27-81$ & A & 62 & 62 & 6 & $s$ \\
\hline NHW & 429 & 411100 & 0713459 & 5 & M & $00 \cdot 00-86$ & H & 14 & 14 & 8 & . \\
\hline NHW & 431 & 410947 & 0713258 & 47 & M & $00-00-87$ & H & 78 & 77 & 4 & s \\
\hline NHW & 432 & 410945 & 0713257 & 39 & M & $08-21-81$ & c & 123 & 123 & 6 & s \\
\hline NHW & 433 & 411231 & 0713416 & 60 & M & $10-10-69$ & c & 112 & 111 & $\cdots$ & s \\
\hline NHW & 434 & 411003 & 0713326 & 85 & M & $06 \cdot 00 \cdot 80$ & $H$ & 110 & 110 & 4 & $s$ \\
\hline NHW & 435 & 410957 & 0713301 & 28 & M & $07 \cdot 28-66$ & c & 93 & 93 & - & $s$ \\
\hline NHW & 436 & 410949 & 0713350 & 115 & M & $06-14-83$ & A & 195 & 195 & 6 & s \\
\hline NHW & 437 & 411055 & 0713601 & 55 & M & $07-21-77$ & c & 62 & 62 & 6 & $s$ \\
\hline NHW & 438 & 411033 & 0713508 & 75 & M & $08-24-83$ & A & 108 & 108 & 6 & s \\
\hline NHW & 440 & 411048 & 0713430 & 18 & M & $09-09-81$ & A & 42 & 42 & 6 & s \\
\hline NHW & 442 & 410943 & 0713251 & 73 & M & $07-27 \cdot 66$ & c & 100 & 100 & 6 & s \\
\hline NHW & 443 & 410952 & 0713343 & 105 & M & $08-31-82$ & A & 202 & 202 & 6 & s \\
\hline NHW & 445 & 411055 & 0713601 & 55 & M & - & D & 18 & 18 & 30 & c \\
\hline NHW & 449 & 410949 & 0713304 & 160 & M & $09-22-83$ & A & 73 & 73 & .. & s \\
\hline NHW & 450 & 410900 & 0713426 & 120 & M & $05-11-71$ & c & 140 & 140 & 6 & $s$ \\
\hline NHW & 451 & 410932 & 0713258 & 120 & M & $11-03-76$ & c & 220 & 220 & .. & s \\
\hline NHW & 452 & 410923 & 0713314 & 158 & M & $08-27-70$ & $c$ & 235 & 235 & 6 & s \\
\hline NHW & 453 & 410919 & 0713314 & 145 & M & $06-25 \cdot 74$ & c & 113 & 113 & 6 & s \\
\hline NHW & 455 & 410953 & 0713603 & 100 & M & $07-28-70$ & c & 162 & 162 & 6 & $s$ \\
\hline NHW & 461 & 411021 & 0713354 & 40 & M & $10-08-86$ & A & 73 & 73 & 6 & s \\
\hline NHW & 463 & 411007 & 0713636 & 18 & M & $07 \cdot 14-88$ & $H$ & 65 & 63 & 4 & $s$ \\
\hline NHW & 465 & 411305 & 0713342 & 69 & M & $06-10 \cdot 66$ & c & 89 & 88 & 6 & $s$ \\
\hline NHW & 466 & 411044 & 0713412 & 17 & M & $07-23 \cdot 63$ & c & 61 & 61 & 6 & s \\
\hline NHW & 467 & 411042 & 0713415 & 20 & M & $06-15-67$ & c & 53 & 53 & 6 & s \\
\hline NHW & 468 & 411018 & 0713321 & 40 & M & $00 \cdot 00-87$ & $z$ & 128 & 128 & 4 & - \\
\hline NHW & 469 & 411010 & 0713554 & 100 & M & $07-26-88$ & H & 183 & 97 & 4 & $s$ \\
\hline & & & & & & -. & $\cdot$ & & & $\cdots$ & - \\
\hline NHW & 501 & 411009 & 0713320 & 65 & M & $07 \cdot 16 \cdot 81$ & A & 113 & 113 & 6 & s \\
\hline & & & & & & $-\cdot$ & - & & & -. & - \\
\hline NHW & 502 & 411011 & 0713324 & 58 & M & $07-15-82$ & A & 124 & 124 & 6 & s \\
\hline NHW & 503 & 411013 & 0713329 & $1_{35}$ & M & $07-30-81$ & A & 94 & 93 & 6 & s \\
\hline NHW & 504 & 411008 & 0713333 & 80 & M & $05-10-83$ & A & 82 & 81 & 6 & s \\
\hline
\end{tabular}


Table 1.--Description of selected wells, springs, and test borings--Continued

[-- or -, no data available; 00 in date columns indicates unknown]

\begin{tabular}{|c|c|c|c|c|c|c|c|c|c|c|c|}
\hline & & $\begin{array}{l}\text { TOP OF } \\
\text { OPEN }\end{array}$ & $\begin{array}{l}\text { BOTTOM OF } \\
\text { OPEN }\end{array}$ & WATER & $\begin{array}{r}\text { DATE } \\
\text { WATER }\end{array}$ & $\begin{array}{l}\text { METHOD } \\
\text { WATER }\end{array}$ & $\begin{array}{l}\text { PRIMARY } \\
\text { USE }\end{array}$ & $\begin{array}{l}\text { PRIMARY } \\
\text { USE }\end{array}$ & & & TYPE \\
\hline & $\begin{array}{l}\text { TION } \\
\text { BER }\end{array}$ & $\begin{array}{l}\text { INTERVAL } \\
\text { (FEET) }\end{array}$ & $\begin{array}{l}\text { INTERVAL } \\
\text { (FEET) }\end{array}$ & $\begin{array}{l}\text { LEVEL } \\
\text { (FEET) }\end{array}$ & $\begin{array}{l}\text { LEVEL } \\
\text { MEASURED }\end{array}$ & $\begin{array}{l}\text { LEVEL } \\
\text { MEASURED }\end{array}$ & $\begin{array}{l}\text { OF } \\
\text { SITE }\end{array}$ & $\begin{array}{l}\text { OF } \\
\text { HATER }\end{array}$ & $\begin{array}{c}\text { DISCHARGE } \\
\text { (GPM) }\end{array}$ & $\begin{array}{l}\text { DRAWDOWN } \\
\text { (FEET) }\end{array}$ & OF LOG \\
\hline NHW & 422 & 234 & 239 & 85 & $06-02-88$ & $R$ & $w$ & H & 50 & 144 & $D$ \\
\hline NHW & 424 & 120 & 130 & 33.44 & $06-21-89$ & $\mathbf{s}$ & $u$ & $u$ & 26 & $\cdots$ & D \\
\hline NHW & 425 & 242 & 246 & 132 & $00 \cdot 00 \cdot 70$ & $R$ & $W$ & $P$ & 40 & 31 & $D$ \\
\hline & & $\cdots$ & $\cdots$ & & & & & & 36 & 28 & - \\
\hline & & $\cdots$ & $\cdots$ & & & & & & 80 & $\cdots$ & $\cdot$ \\
\hline NHW & 426 & 134 & 138 & 14 & $11-11-67$ & $R$ & $W$ & $E$ & 35 & 66 & D \\
\hline NHW & 427 & $\cdots$ & $\cdots$ & 8.32 & $03 \cdot 09 \cdot 88$ & $s$ & $U$ & $u$ & $\cdots$ & $\cdots$ & - \\
\hline NHW & 428 & 60 & 62 & 25.8 & $03-09-88$ & $T$ & $W$ & c & 20 & $\cdots$ & D \\
\hline NHW & 429 & $\cdots$ & $\cdots$ & 2.6 & $03 \cdot 09 \cdot 88$ & $T$ & $u$ & $u$ & $\cdots$ & $\cdots$ & D \\
\hline NHW & 431 & 72 & 77 & 20 & $00-00-87$ & $R$ & $W$ & $H$ & .. & $\cdots$ & $D$ \\
\hline NHW & 432 & 120 & 123 & 39 & $08-21-81$ & $R$ & $W$ & H & 40 & $\cdots$ & D \\
\hline NHW & 433 & 109 & 111 & 57 & $10 \cdot 10 \cdot 69$ & $\mathbf{R}$ & $w$ & H & 20 & 8 & D \\
\hline NHW & 434 & $\cdots$ & $\cdots$ & 50 & $06 \cdot 00 \cdot 80$ & $\mathbf{R}$ & $W$ & $P$ & $\cdots$ & - & - \\
\hline NHW & 435 & 89 & 93 & 21 & $07-28 \cdot 66$ & $R$ & $W$ & H & 40 & 0 & $D$ \\
\hline NHW & 436 & 192 & 195 & 108 & $06 \cdot 14 \cdot 83$ & $R$ & $w$ & H & 75 & - & D \\
\hline NHW & 437 & 58 & 62 & 36 & $07 \cdot 21 \cdot 77$ & $R$ & $w$ & H & 10 & $\cdots$ & D \\
\hline NHW & 438 & 105 & 108 & 73.4 & $06 \cdot 29 \cdot 89$ & $T$ & $w$ & $H$ & 25 & $\cdots$ & $D$ \\
\hline NHW & 440 & 39 & 42 & 15 & $09-09 \cdot 81$ & $\mathbf{R}$ & $w$ & H & 20 & . & $D$ \\
\hline NHW & 442 & $\cdots$ & $\cdots$ & 74 & $07-27-66$ & $R$ & $w$ & $\cdot$ & 7 & $\cdots$ & - \\
\hline NHW & 443 & 199 & 202 & 107 & $08-31-82$ & $R$ & $W$ & $P$ & 70 & $\cdots$ & D \\
\hline NHW & 445 & $\cdots$ & $\cdots$ & 8.14 & $06-23-88$ & s & $u$ & $u$ & $\cdots$ & $\cdots$ & - \\
\hline NHW & 449 & 71 & 73 & 20 & $09 \cdot 22 \cdot 83$ & $\mathbf{R}$ & $w$ & H & 14 & $\cdots$ & D \\
\hline NHW & 450 & 138 & 140 & 120 & $05-11-71$ & $\mathbf{R}$ & $w$ & H & 25 & 13 & D \\
\hline NHW & 451 & 216 & 220 & 107 & $11-03-76$ & $R$ & $w$ & H & 6 & 53 & D \\
\hline NHW & 452 & 232 & 235 & 150 & $08-27 \cdot 70$ & $\mathbf{R}$ & $W$ & H & 12 & 30 & D \\
\hline NHW & 453 & 110 & 113 & 84 & $06 \cdot 25 \cdot 74$ & $R$ & $w$ & H & 20 & $\cdots$ & D \\
\hline NHW & 455 & 160 & 162 & 95 & $07-28-70$ & $R$ & $w$ & H & 30 & 12 & D \\
\hline NHW & 461 & 71 & 73 & 30 & $10-08 \cdot 86$ & $R$ & $w$ & H & 20 & $\cdots$ & D \\
\hline NHW & 463 & 58 & 63 & $\cdots$ & -. & - & $w$ & H & 2 & $\cdots$ & $D$ \\
\hline NHW & 465 & 84 & 88 & 69 & $06-10-66$ & $\mathbf{R}$ & $W$ & H & 38 & $\cdots$ & $D$ \\
\hline NHW & 466 & 57 & 61 & 14 & $07-23 \cdot 63$ & $\mathbf{R}$ & $w$ & H & 40 & $\cdots$ & D \\
\hline NHW & 467 & 49 & 53 & 17 & $06-15-67$ & $\mathbf{R}$ & $w$ & $P$ & 40 & 24 & 0 \\
\hline NHW & 468 & $\cdots$ & $\cdots$ & 39.46 & $06 \cdot 19 \cdot 89$ & $\mathbf{s}$ & $w$ & $u$ & $\cdots$ & $\cdots$ & . \\
\hline NHW & 469 & 92 & 97 & 75.3 & $07-28 \cdot 88$ & $T$ & $w$ & H & $\cdots$ & $\cdots$ & - \\
\hline & & $\cdots$ & $\cdots$ & & & & & & $\cdots$ & $\cdots$ & $D$ \\
\hline NHW & 501 & 110 & 113 & 28 & $07-16-81$ & $R$ & $w$ & $\mathbf{P}$ & 50 & $\cdots$ & $D$ \\
\hline & & $\cdots$ & $\cdots$ & & & & & & 25 & 22 & - \\
\hline NHW & 502 & 121 & 124 & 55 & $07-15-82$ & $R$ & $w$ & H & 100 & $\cdots$ & D \\
\hline NHW & 503 & 90 & 93 & 26 & $07-30-81$ & $\mathbf{R}$ & $w$ & $\mathbf{P}$ & 75 & $\cdots$ & D \\
\hline NHW & 504 & 78 & 81 & 50 & $05-10-83$ & $R$ & $w$ & $\mathbf{P}$ & 12 & $\cdots$ & D \\
\hline
\end{tabular}


Table 1.--Description of selected wells, springs, and test borings--Continued

[-- or -, no data available; 00 in date columns indicates date unknown]

\begin{tabular}{|c|c|c|c|c|c|c|c|c|c|c|c|}
\hline & & & & ALTITUDE & & & & & & DIAMETER & \\
\hline & & & & OF LAND & METHOD & & METHOD & DEPTH & DEPTH & OF & TYPE \\
\hline STA & TION & & & SURFACE & ALTITUDE & DATE OF & CONST - & DRILLED & OF WELL & CASING & OF \\
\hline NUM & BER & LATITUDE & LONGI TUDE & (FEET) & DETERMINED & CONSTRUCTION & RUCTED & (FEET) & (FEET) & (IN) & FINISH \\
\hline NHW & 505 & 410921 & 0713300 & 115 & $M$ & $05-08-79$ & A & 234 & 234 & 6 & $\mathbf{s}$ \\
\hline & & & & & & $\cdots$ & - & & & -. & - \\
\hline NHW & 506 & 410917 & 0713301 & 135 & M & $05-10-79$ & A & 207 & 207 & 6 & $\mathbf{s}$ \\
\hline NHW & 508 & 410920 & 0713309 & 140 & M & $07-10-69$ & c & 144 & 143 & 6 & s \\
\hline NHW & 509 & 410927 & 0713257 & 115 & M & $09-22 \cdot 81$ & A & 215 & 214 & 6 & s \\
\hline NHW & 511 & 411020 & 0713319 & 10 & $M$ & $05-26-82$ & A & 51 & 51 & 6 & s \\
\hline NHW & 512 & 410928 & 0713301 & 125 & M & $09-22-86$ & A & 245 & 245 & 6 & $\mathbf{s}$ \\
\hline NHW & 514 & 410950 & 0713252 & 40 & M & 06.06 .67 & C & 150 & 150 & 6 & $\mathbf{s}$ \\
\hline NHW & 515 & 410951 & 0713255 & 40 & M & $08-06-85$ & A & 149 & 149 & 6 & $s$ \\
\hline NHW & 516 & 410952 & 0713254 & 43 & M & $06-12-81$ & A & 54 & 54 & 6 & $s$ \\
\hline NHW & 517 & 411002 & 0713319 & 80 & M & $07-17-81$ & A & 127 & 127 & 6 & s \\
\hline NHW & 519 & 410958 & 0713313 & 160 & M & $08-00-87$ & A & 121 & 121 & 6 & $s$ \\
\hline NHW & 520 & 410948 & 0713312 & 98 & M & $09-11.69$ & c & 103 & 103 & 6 & $\mathbf{s}$ \\
\hline NHW & 521 & 410949 & 0713306 & 85 & M & $09-04-80$ & A & 124 & 124 & 6 & $s$ \\
\hline NHW & 522 & 410951 & 0713300 & 50 & M & $06-20-74$ & C & 79 & 79 & 6 & $s$ \\
\hline NHW & 523 & 410940 & 0713310 & 90 & M & $09-04 \cdot 79$ & A & 84 & 84 & 6 & s \\
\hline & & & & & & $\cdots$ & - & & & .. & - \\
\hline NHW & 524 & 410938 & 0713313 & 115 & M & $05-20-68$ & c & 193 & 193 & 6 & s \\
\hline NHW & 526 & 410939 & 0713307 & $1_{127}$ & M & $07-02-81$ & A & 219 & 219 & 6 & $\mathbf{s}$ \\
\hline NHW & 527 & 410937 & 0713303 & 85 & M & $09-14 \cdot 70$ & c & 186 & 186 & 6 & $s$ \\
\hline & & & & & & .. & - & & & 5 & - \\
\hline NHW & 528 & 410916 & 0713325 & 160 & M & $08-23-85$ & A & 190 & 190 & 6 & $s$ \\
\hline NHW & 529 & 410931 & 0713328 & 159 & M & $08 \cdot 05 \cdot 80$ & A & 144 & 144 & 6 & $\mathbf{s}$ \\
\hline NHW & 530 & 410934 & 0713338 & 145 & M & $08-21-85$ & A & 164 & 164 & 6 & $\mathbf{s}$ \\
\hline NHW & 531 & 410935 & 0713339 & 145 & M & $\cdots$ & $\cdot$ & 151 & 151 & $\cdots$ & s \\
\hline & & & & & & $-\cdot$ & - & & & .. & - \\
\hline NHW & 532 & 410923 & 0713327 & 145 & M & $06-11-71$ & c & 111 & 111 & 6 & s \\
\hline NHW & 533 & 410935 & 0713329 & 144 & M & $05 \cdot 31-73$ & C & 155 & 155 & 6 & s \\
\hline NHW & 534 & 410938 & 0713335 & 137 & M & $05-23-75$ & c & 128 & 128 & 6 & s \\
\hline NHW & 535 & 410927 & 0713432 & 154.42 & $\mathbf{L}$ & $08-07-68$ & c & 163 & 163 & 6 & s \\
\hline NHW & 537 & 410915 & 0713401 & 151 & M & $08-20-87$ & H & 182 & 182 & 4 & $s$ \\
\hline NHW & 538 & 410919 & 0713424 & ${ }^{1} 164$ & A & $07-27 \cdot 72$ & c & 120 & 120 & 6 & s \\
\hline NHW & 539 & 410922 & 0713421 & 140 & $M$ & $06-16-67$ & $c$ & 53 & 52 & 6 & s \\
\hline NHW & 540 & 410912 & 0713431 & 170 & M & $08-20-79$ & A & 231 & 231 & 6 & s \\
\hline & & & & & & $\cdots$ & $\cdot$ & & & -. & $\cdot$ \\
\hline NHW & 541 & 410920 & 0713441 & 110 & M & $04-15-66$ & $c$ & 83 & 83 & 6 & $s$ \\
\hline NHW & 542 & 410909 & 0713435 & ${ }^{1} 154$ & A & $10-15-71$ & c & 216 & 216 & 6 & 0 \\
\hline NHW & 543 & 410907 & 0713440 & ${ }^{1} 130$ & A & $06-04-85$ & A & 225 & 225 & 6 & s \\
\hline NHW & 544 & 410902 & 0713435 & 120 & M & $09-09-66$ & c & 93 & 93 & 6 & s \\
\hline NHW & 545 & 410902 & 0713434 & 115 & $M$ & $09-29-70$ & c & 146 & 145 & 6 & s \\
\hline
\end{tabular}


Table 1.--Description of selected wells, springs, and test borings--Continued [-- or -, no data available; 00 in date columns indicates unknown]

\begin{tabular}{|c|c|c|c|c|c|c|c|c|c|c|c|}
\hline \multicolumn{2}{|c|}{$\begin{array}{l}\text { STATION } \\
\text { NUMBER }\end{array}$} & $\begin{array}{l}\text { TOP OF } \\
\text { OPEN } \\
\text { INTERVAL } \\
\text { (FEET) }\end{array}$ & $\begin{array}{l}\text { BOTTOM OF } \\
\text { OPEN } \\
\text { INTERVAL } \\
\text { (FEET) }\end{array}$ & $\begin{array}{l}\text { WATER } \\
\text { LEVEL } \\
\text { (FEET) }\end{array}$ & $\begin{array}{c}\text { DATE } \\
\text { WATER } \\
\text { LEVEL } \\
\text { MEASURED }\end{array}$ & $\begin{array}{l}\text { METHOD } \\
\text { WATER } \\
\text { LEVEL } \\
\text { MEASURED }\end{array}$ & $\begin{array}{c}\text { PRIMARY } \\
\text { USE } \\
\text { OF } \\
\text { SITE }\end{array}$ & $\begin{array}{c}\text { PRIMARY } \\
\text { USE } \\
\text { OF } \\
\text { WATER }\end{array}$ & $\begin{array}{c}\text { DISCHARGE } \\
\text { (GPM) }\end{array}$ & $\begin{array}{l}\text { DRAWDOWN } \\
\text { (FEET) }\end{array}$ & $\begin{array}{c}\text { TYPE } \\
\text { OF LOG } \\
\text { AVAILABLE }\end{array}$ \\
\hline NHW & 505 & 231 & 234 & 138 & $05-08-79$ & $\mathbf{R}$ & $W$ & H & 40 & $\cdots$ & D \\
\hline & & $\cdots$ & -- & & & & & & 10 & 12 & - \\
\hline NHW & 506 & 204 & 207 & 130 & $05-10-79$ & $\mathbf{R}$ & $W$ & H & 10 & 20 & D \\
\hline NHW & 508 & 140 & 143 & 113 & $07-10-69$ & $\mathbf{R}$ & $W$ & H & 28 & 5 & $D$ \\
\hline NHW & 509 & 211 & 214 & 100 & $09 \cdot 22 \cdot 81$ & $\mathbf{R}$ & $W$ & $H$ & 95 & $\cdots$ & $D$ \\
\hline NHW & 511 & 49 & 51 & 10 & $05-26-82$ & $\mathbf{R}$ & $z$ & $\mathbf{U}$ & 40 & $\cdots$ & $D$ \\
\hline NHW & 512 & 242 & 245 & 120 & $09 \cdot 22 \cdot 86$ & $\mathbf{R}$ & $W$ & H & 50 & $\cdots$ & $D$ \\
\hline NHW & 514 & 146 & 150 & 34 & $06-06 \cdot 67$ & $\mathbf{R}$ & $W$ & H & 35 & 12 & D \\
\hline NHW & 515 & 146 & 149 & 50 & $08 \cdot 06-85$ & $\mathbf{R}$ & $W$ & H & 70 & $\cdots$ & $D$ \\
\hline NHW & 516 & 50 & 54 & $\cdots$ & $\cdots$ & $\cdot$ & $W$ & H & 4 & $\cdots$ & $D$ \\
\hline NHW & 517 & 124 & 127 & 58 & $07-17-81$ & $\mathbf{R}$ & $W$ & $P$ & 100 & -. & D \\
\hline NHW & 519 & $\cdots$ & - & 30 & $08-00-87$ & $\mathbf{R}$ & $W$ & H & 35 & $\cdots$ & $\cdot$ \\
\hline NHW & 520 & 100 & 103 & 68 & $09-11 \cdot 69$ & $\mathbf{R}$ & $W$ & H & 6 & $\cdots$ & $D$ \\
\hline NHW & 521 & 121 & 124 & 72 & $09-04-80$ & $\mathbf{R}$ & $W$ & H & 40 & $\cdots$ & D \\
\hline NHW & 522 & 76 & 79 & 22 & $06-20-74$ & $\mathbf{R}$ & $w$ & H & 40 & $\cdots$ & D \\
\hline NHW & 523 & 81 & 84 & 38 & $09-04-79$ & $\mathbf{R}$ & $w$ & H & 37 & $\cdots$ & D \\
\hline & & $\cdots$ & $\cdots$ & & & & & & 8 & 7 & - \\
\hline NHW & 524 & 190 & 193 & 96 & $05-20-68$ & $\mathbf{R}$ & $w$ & H & 35 & 1 & $D$ \\
\hline NHW & 526 & 218 & 219 & 111 & $07-02-81$ & $\mathbf{R}$ & $W$ & H & 10 & 39 & D \\
\hline NHW & 527 & 181 & 186 & 85 & $09-14 \cdot 70$ & $\mathbf{R}$ & $W$ & H & 14 & 45 & $D$ \\
\hline & & $\cdots$ & $\cdots$ & & & & & & $\cdots$ & $\cdots$ & $\cdot$ \\
\hline NHW & 528 & 188 & 190 & $\cdots$ & $\cdots$ & $\cdot$ & $W$ & H & 20 & $\cdots$ & $D$ \\
\hline NHW & 529 & 141 & 144 & 121 & $08-05-80$ & $\mathbf{R}$ & $W$ & H & 20 & 19 & $D$ \\
\hline NHW & 530 & 161 & 164 & $\cdots$ & -- & $\cdot$ & $W$ & H & 45 & 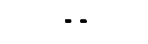 & $D$ \\
\hline NHW & 531 & $\cdots$ & $\cdots$ & 108 & $11 \cdot 03 \cdot 70$ & $\mathbf{R}$ & U & $U$ & 7 & 32 & $\cdot$ \\
\hline & & $\cdots$ & $\cdots$ & & & & & & 6 & $\cdots$ & - \\
\hline NHW & 532 & 107 & 111 & 85 & $06-11 \cdot 71$ & $\mathbf{R}$ & $w$ & H & 35 & 15 & $D$ \\
\hline NHW & 533 & 152 & 155 & 105 & $05-31-73$ & $\mathbf{R}$ & $W$ & H & 14 & $\cdots$ & D \\
\hline NHW & 534 & 126 & 128 & 94 & $05-23-75$ & $\mathbf{R}$ & $W$ & $H$ & 5 & $-\cdot$ & $D$ \\
\hline NHW & 535 & 159 & 163 & 123 & $08-07-68$ & $\mathbf{R}$ & $W$ & H & 15 & 10 & $D$ \\
\hline NHW & 537 & - & $-\cdot$ & 151 & $08 \cdot 20-87$ & $\mathbf{R}$ & $W$ & H & 20 & $\cdots$ & - \\
\hline NHW & 538 & 117 & 120 & 99 & $07-27-72$ & $\mathbf{R}$ & $W$ & H & 16 & 21 & $D$ \\
\hline NHW & 539 & 48 & 52 & 9 & $06-16-67$ & $\mathbf{R}$ & $W$ & H & 10 & 43 & D \\
\hline NHW & 540 & 228 & 231 & 165 & $08-20 \cdot 79$ & $\mathbf{R}$ & $W$ & H & 22 & $\cdots$ & $D$ \\
\hline & & $\cdots$ & $\cdots$ & & & & & & 8 & 15 & $\cdot$ \\
\hline NHW & 541 & 79 & 83 & 55 & $04-15-66$ & $\mathbf{R}$ & $w$ & H & 30 & -. & $D$ \\
\hline NHW & 542 & $\cdots$ & $-\cdot$ & 152 & $10-15-71$ & $\mathbf{R}$ & $W$ & H & 30 & $\cdots$ & $D$ \\
\hline NHW & 543 & 222 & 225 & 150 & $06-04-85$ & $\mathbf{R}$ & $W$ & H & 30 & $\cdots$ & $D$ \\
\hline NHW & 544 & 90 & 93 & 68 & $09-09-66$ & $\mathbf{R}$ & $W$ & H & 30 & 11 & $D$ \\
\hline NHW & 545 & 142 & 145 & -. & $\cdots$ & - & $W$ & H & 25 & $\cdots$ & $D$ \\
\hline
\end{tabular}


Table 1.--Description of selected wells, springs, and test borings--Continued

$$
\text { [ } \cdots \text { or } \cdot \text {, no data available; } 00 \text { in date columns indicates date unknown] }
$$

\begin{tabular}{|c|c|c|c|c|c|c|c|c|c|c|c|}
\hline & & & & ALTITUDE & & & & & & DIAMETER & \\
\hline & & & & OF LAND & METHOD & & METHOD & DEPTH & DEPTH & & TYPE \\
\hline STA & & & & SURFACE & ALTITUDE & DATE OF & CONST - & DRILLED & OF WELL & CASING & OF \\
\hline NHW & 546 & 410859 & 0713430 & 95 & $M$ & $05 \cdot 27-86$ & A & 143 & 143 & 6 & s \\
\hline NHW & 547 & 410906 & 0713427 & 143 & $M$ & $09 \cdot 17 \cdot 79$ & A & 224 & 223 & 6 & $\mathbf{s}$ \\
\hline NHW & 548 & 410902 & 0713414 & 121.12 & $\mathrm{~L}$ & $07 \cdot 27 \cdot 77$ & C & 161 & 158 & 6 & s \\
\hline NHW & 549 & 410902 & 0713413 & 130.13 & $L$ & $05 \cdot 07 \cdot 80$ & A & 130 & 130 & 6 & $\mathbf{s}$ \\
\hline NHW & 550 & 410905 & 0713414 & 115 & M & $09 \cdot 16-65$ & C & 96 & 96 & 6 & s \\
\hline NHW & 551 & 410904 & 0713407 & 135 & $M$ & $09-04-74$ & C & 104 & 104 & 6 & s \\
\hline NHW & 552 & 410901 & 0713358 & 147 & A & $10-11-66$ & C & 194 & 194 & 6 & s \\
\hline NHW & 553 & 410906 & 0713358 & 155 & M & $10-18-69$ & C & 131 & 131 & 6 & s \\
\hline NHW & 554 & 410906 & 0713400 & $1_{152.49}$ & $L$ & $05-14-80$ & A & 200 & 199 & 6 & s \\
\hline & & & & & & $\cdots$ & $\cdot$ & & & $\cdots$ & $\cdot$ \\
\hline NHW & 555 & 410911 & 0713400 & 150 & A & $05-20 \cdot 70$ & C & 145 & 145 & 6 & s \\
\hline NHW & 556 & 410910 & 0713358 & 167.82 & L & $09 \cdot 09 \cdot 83$ & A & 222 & 222 & 6 & s \\
\hline NHW & 557 & 410917 & 0713356 & 171.62 & $L$ & $06-30-70$ & C & 260 & 260 & 5 & s \\
\hline NHW & 558 & 410909 & 0713353 & 177 & $M$ & $08-23-67$ & C & 206 & 206 & 6 & s \\
\hline NHW & 559 & 410909 & 0713347 & 160 & $M$ & $05 \cdot 31 \cdot 74$ & c & 217 & 217 & 6 & s \\
\hline & & & & & & $\cdots$ & $\cdot$ & & & $\cdots$ & $\cdot$ \\
\hline NHW & 560 & 410913 & 0713349 & 175 & $M$ & $07-12 \cdot 72$ & C & 248 & 248 & 6 & 0 \\
\hline NHW & 561 & 410913 & 0713353 & 179 & $M$ & $06 \cdot 13 \cdot 73$ & C & 240 & 240 & 6 & s \\
\hline NHW & 562 & 410915 & 0713355 & 160 & $M$ & $07-22-80$ & A & 201 & 200 & 6 & s \\
\hline NHW & 563 & 410917 & 0713345 & 175 & M & $08-31-67$ & C & 206 & 206 & 6 & $\mathbf{s}$ \\
\hline NHW & 564 & 410924 & 0713356 & 150.14 & $L$ & $09 \cdot 14 \cdot 82$ & A & 114 & 114 & 6 & s \\
\hline & & & & & & $\cdots$ & - & & & $\cdots$ & $\cdot$ \\
\hline NHW & 565 & 410928 & 0713353 & 163 & $M$ & $05-15 \cdot 69$ & C & 108 & 104 & 6 & s \\
\hline NHW & 566 & 410929 & 0713354 & 151 & A & $09-15-82$ & A & 114 & 114 & 6 & $\mathbf{s}$ \\
\hline NHW & 567 & 410937 & 0713346 & 135 & $M$ & $07-13-82$ & A & 164 & 164 & 6 & $\mathbf{s}$ \\
\hline & & & & & & -. & - & & & $\cdots$ & - \\
\hline NHW & 569 & 410932 & 0713341 & 162 & $M$ & $05-17-79$ & A & 162 & 162 & 6 & s \\
\hline NHW & 570 & 410931 & 0713344 & 165 & $M$ & $10 \cdot 08 \cdot 71$ & C & 165 & 164 & 6 & $\mathbf{s}$ \\
\hline NHW & 571 & 410925 & 0713334 & 172 & $M$ & $05-29-86$ & A & 267 & 267 & 6 & s \\
\hline NHW & 572 & 410950 & 0713347 & 110 & $M$ & $11 \cdot 13-72$ & C & 146 & 146 & 6 & $\mathbf{s}$ \\
\hline & & & & & & $-\cdot$ & - & & & $\cdots$ & - \\
\hline NHW & 573 & 410951 & 0713348 & 118 & $M$ & $06-30-82$ & A & 190 & 190 & 6 & s \\
\hline NHW & 574 & 410950 & 0713352 & 1115 & $M$ & $09-07-83$ & A & 194 & 194 & 6 & s \\
\hline NHW & 575 & 410905 & 0713353 & 150 & $M$ & $06-08 \cdot 76$ & C & 195 & 195 & 6 & $\mathbf{s}$ \\
\hline NHW & 576 & 411318 & 0713354 & 25 & $M$ & $07-10-75$ & C & 51 & 51 & 6 & $\mathbf{s}$ \\
\hline NHW & 577 & 410932 & 0713430 & 130.61 & L & $08 \cdot 21 \cdot 68$ & C & 93 & 93 & $\cdots$ & s \\
\hline NHW & 578 & 411023 & 0713536 & 170 & $M$ & $05 \cdot 11 \cdot 89$ & $\cdot$ & 270 & 270 & $\cdots$ & s \\
\hline & & & & & & $\cdots$ & $\cdot$ & & & $\cdots$ & $\cdot$ \\
\hline NHW & 581 & 411125 & 0713516 & 5 & $M$ & $00-00 \cdot 87$ & D & 10 & 10 & 36 & 0 \\
\hline
\end{tabular}


Table 1.--Description of selected wells, springs, and test borings--Continued [-. or -, no data available; 00 in date columns indicates unknown]

\begin{tabular}{|c|c|c|c|c|c|c|c|c|c|c|c|}
\hline & & $\begin{array}{l}\text { TOP OF } \\
\text { OPEN }\end{array}$ & $\begin{array}{l}\text { BOTTOM OF } \\
\text { OPEN }\end{array}$ & WATER & $\begin{array}{r}\text { DATE } \\
\text { WATER }\end{array}$ & $\begin{array}{l}\text { METHOD } \\
\text { WATER }\end{array}$ & $\begin{array}{l}\text { PRIMARY } \\
\text { USE }\end{array}$ & $\begin{array}{l}\text { PRIMARY } \\
\text { USE }\end{array}$ & & & TYPE \\
\hline STA & IION & INTERVAL & INTERVAL & LEVEL & LEVEL & LEVEL & OF & OF & DISCHARGE & DRAWDOWN & OF LOG \\
\hline NUME & BER & (FEET) & (FEET) & (FEET) & MEASURED & MEASURED & SITE & WATER & (GPM) & (FEET) & AVAILABLE \\
\hline NHW & 546 & 141 & 143 & 120 & $05-27 \cdot 86$ & $\mathbf{R}$ & $\mathbf{W}$ & $H$ & 12 & $\cdots$ & D \\
\hline NHW & 547 & 220 & 223 & 168 & $09-17-79$ & $R$ & $W$ & $H$ & 40 & $\cdots$ & D \\
\hline NHW & 548 & 155 & 158 & 120 & $07-27-77$ & $R$ & $W$ & $H$ & 6 & $\cdots$ & D \\
\hline NHW & 549 & 127 & 130 & 75 & $05-07-80$ & $\mathbf{R}$ & $W$ & $H$ & 15 & $\cdots$ & D \\
\hline NHW & 550 & 92 & 96 & 58 & $06-16-65$ & $\mathbf{R}$ & $W$ & $H$ & 35 & $-\cdot$ & D \\
\hline NHW & 551 & 101 & 104 & 60 & $09 \cdot 04 \cdot 74$ & $\mathbf{R}$ & $W$ & $H$ & 12 & 20 & D \\
\hline NHW & 552 & 190 & 194 & 130 & $10-11-66$ & $\mathbf{R}$ & $W$ & H & 8 & 40 & D \\
\hline NHW & 553 & 128 & 131 & 104 & $10-18-69$ & $\mathbf{R}$ & $W$ & H & 20 & 11 & D \\
\hline NHW & 554 & 196 & 199 & 150 & $05-14-80$ & $\mathbf{R}$ & $W$ & $H$ & 25 & 49 & D \\
\hline & & $\cdots$ & $\cdots$ & & & & & & 7 & 20 & $\cdot$ \\
\hline NHW & 555 & 142 & 145 & 117 & $05-20 \cdot 70$ & $\mathbf{R}$ & $W$ & $H$ & 12 & 9 & D \\
\hline NHW & 556 & 219 & 222 & 170 & $09 \cdot 09 \cdot 83$ & $\mathbf{R}$ & $W$ & $H$ & 40 & $\cdots$ & D \\
\hline NHW & 557 & 256 & 260 & 170 & $06-30 \cdot 70$ & $R$ & $W$ & $H$ & 18 & 2 & $D$ \\
\hline NHW & 558 & 204 & 206 & 177 & $08-23-67$ & $\mathbf{R}$ & $W$ & $H$ & 18 & $\cdots$ & $D$ \\
\hline NHW & 559 & 214 & 217 & 160 & $05 \cdot 31-74$ & $R$ & $W$ & $H$ & 15 & 57 & $D$ \\
\hline & & $\cdots$ & $\cdots$ & & & & & & 7 & 20 & $\cdot$ \\
\hline NHW & 560 & 248 & $\cdots$ & 177 & $07-12-72$ & $\mathbf{R}$ & $W$ & $H$ & 15 & $-\cdot$ & $D$ \\
\hline NHW & 561 & 238 & 240 & 179 & $06-13 \cdot 73$ & $\mathbf{R}$ & $W$ & $H$ & 8 & 19 & D \\
\hline NHW & 562 & 196 & 200 & 168 & $07-22-80$ & $\mathbf{R}$ & $W$ & $H$ & 12 & $\cdots$ & D \\
\hline NHW & 563 & 202 & 206 & 164 & $08-31-67$ & $\mathbf{R}$ & $W$ & - & 9 & 16 & D \\
\hline NHW & 564 & 111 & 114 & 58 & $09 \cdot 14 \cdot 82$ & $\mathbf{R}$ & $W$ & $H$ & 40 & -. & D \\
\hline & & $\cdots$ & $\cdots$ & & & & & & 12 & 22 & - \\
\hline NHW & 565 & 101 & 104 & 78 & $05 \cdot 15 \cdot 69$ & $\mathbf{R}$ & W & $H$ & 8 & $\cdots$ & D \\
\hline NHW & 566 & 111 & 114 & 60 & $09 \cdot 15 \cdot 82$ & $\mathbf{R}$ & $w$ & H & 29 & $\cdots$ & $D$ \\
\hline NHW & 567 & 161 & 164 & 100 & $07-13-82$ & $R$ & $W$ & H & 75 & $\cdots$ & D \\
\hline & & $\cdots$ & $\cdots$ & & & & & & 5 & 10 & - \\
\hline NHW & 569 & 158 & 162 & 130 & $05-17-79$ & $\mathbf{R}$ & $W$ & $H$ & 50 & $\cdots$ & D \\
\hline NHW & 570 & 162 & 164 & 136 & $10-08-71$ & $\mathbf{R}$ & W & $H$ & 5 & -. & $D$ \\
\hline NHW & 571 & 240 & 267 & 162 & $05-29-86$ & $\mathbf{R}$ & $W$ & $H$ & 40 & $\cdots$ & $\cdot$ \\
\hline NHW & 572 & 143 & 146 & 70 & $11-13-72$ & $\mathbf{R}$ & $W$ & $H$ & 20 & $\cdots$ & D \\
\hline & & $\cdots$ & $\cdots$ & & & & & & 12 & 20 & - \\
\hline NHW & 573 & 187 & 190 & 115 & $06-30-82$ & $\mathbf{R}$ & $W$ & $H$ & 50 & $\cdots$ & D \\
\hline NHW & 574 & 190 & 194 & 115 & $09-07-83$ & $\mathbf{R}$ & $W$ & $H$ & 50 & $\cdots$ & D \\
\hline NHW & 575 & 192 & 195 & 165 & $06 \cdot 08 \cdot 76$ & $R$ & $w$ & $H$ & 5 & $\cdots$ & D \\
\hline NHW & 576 & 48 & 51 & 20 & $07-10 \cdot 75$ & $\mathbf{R}$ & $w$ & $H$ & 45 & - & D \\
\hline NHW & 577 & 89 & 93 & 56 & $08 \cdot 21 \cdot 68$ & $\mathbf{R}$ & $w$ & $H$ & 10 & $\cdots$ & D \\
\hline NHW & 578 & 266 & 270 & $\cdots$ & $\cdots$ & $\cdot$ & $W$ & $H$ & $\cdots$ & $\cdots$ & $D$ \\
\hline & & $\cdots$ & $\cdots$ & & & & & & $\cdots$ & $-\cdot$ & $D$ \\
\hline NHW & 581 & $\cdots$ & $\cdots$ & $\cdots$ & $\therefore$ & - & $w$ & $H$ & 2 & $\cdots$ & $\cdot$ \\
\hline
\end{tabular}


Table 1.-Description of selected wells, springs, and test borings--Continued

[-. or -, no data available; 00 in date columns indicates date unknown]

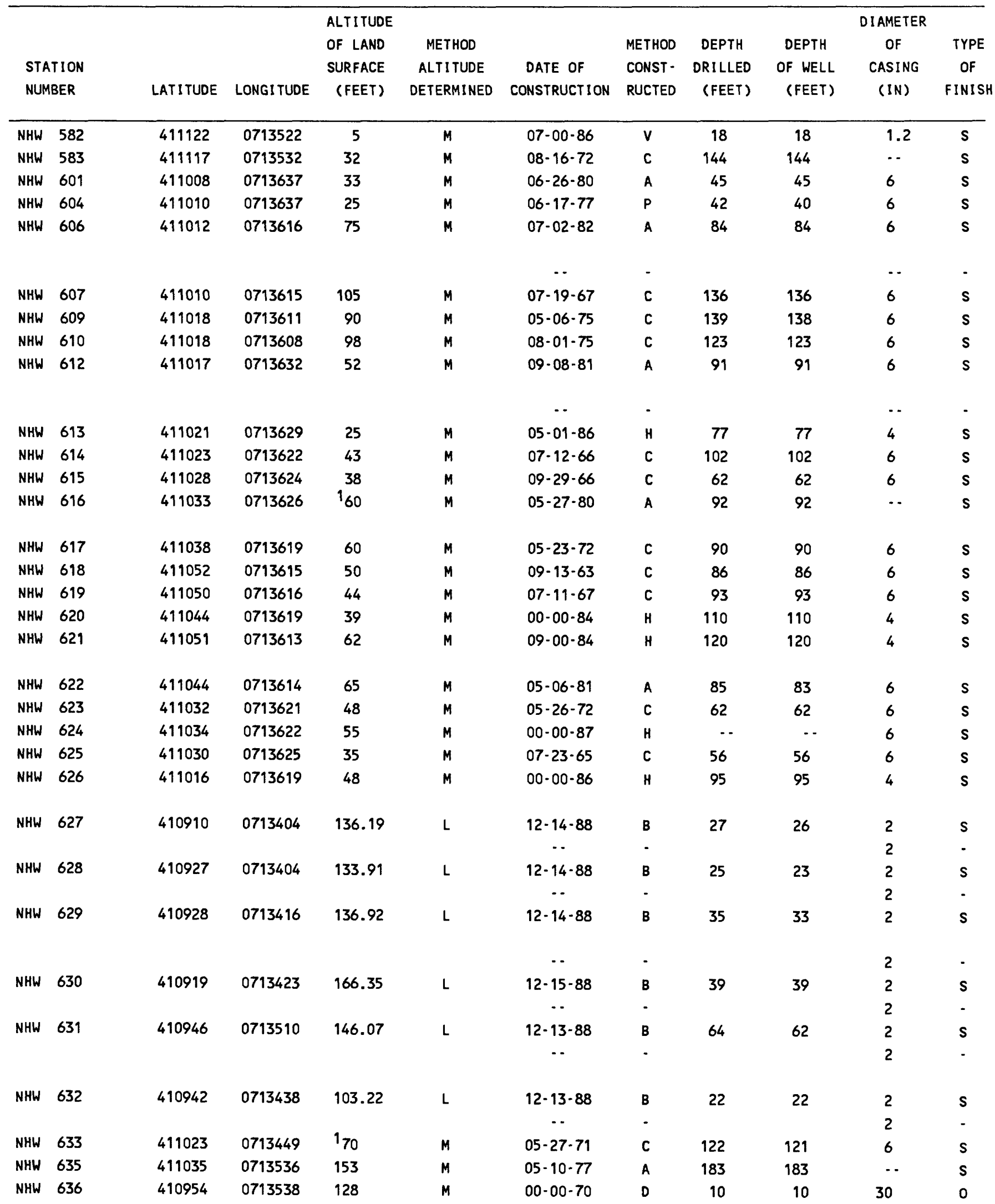


Table 1.--Description of selected wells, springs, and test borings--Continued

$$
\text { [-- or -, no data available; } 00 \text { in date columns indicates unknown] }
$$

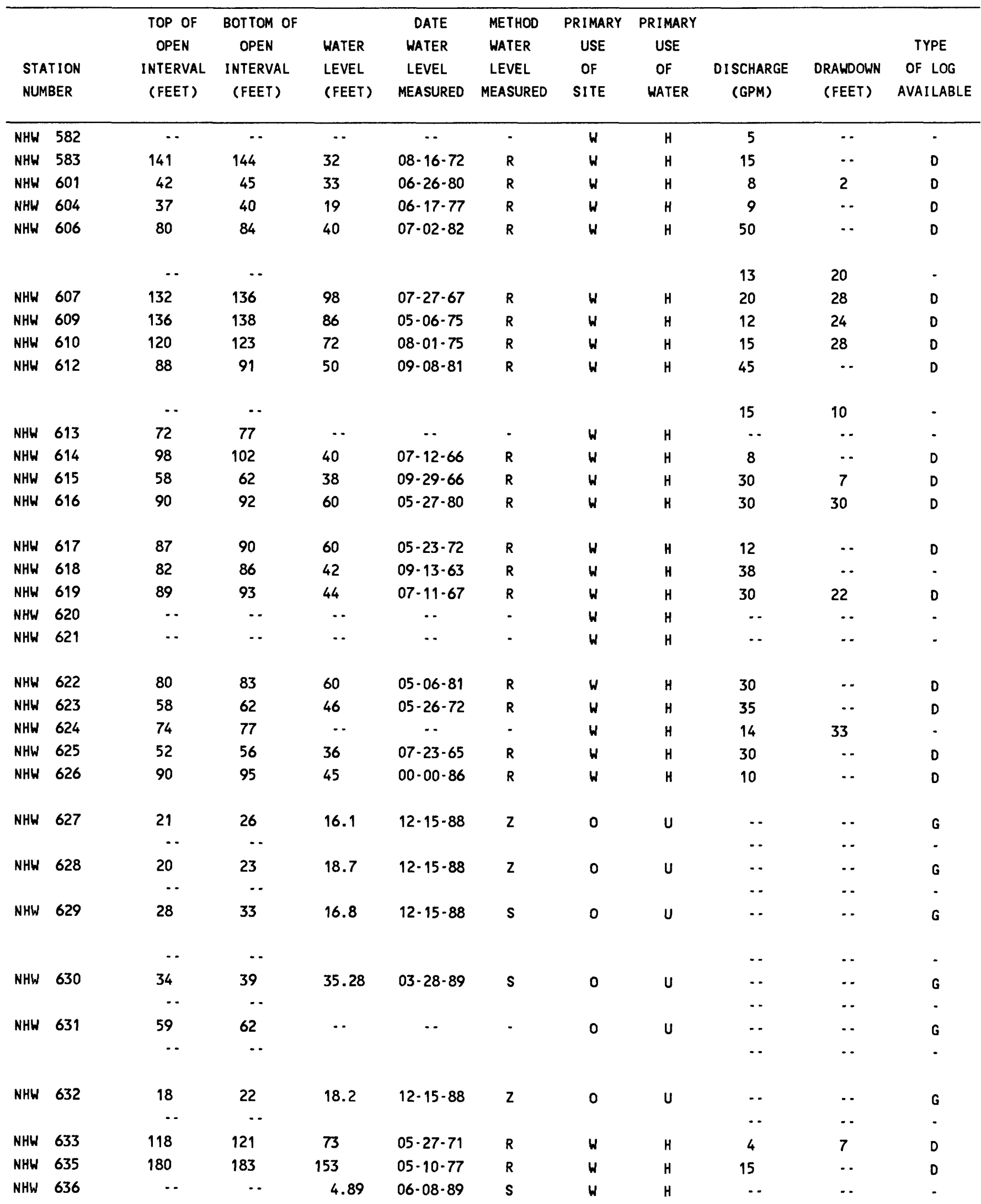


Table 1.--Description of selected wells, springs, and test borings--Cont inued

$$
\text { [- or -, no data available; } 00 \text { in date columns indicates date unknown] }
$$

\begin{tabular}{|c|c|c|c|c|c|c|c|c|c|c|c|}
\hline & & & & ALTITUDE & & & & & & DIAMETER & \\
\hline & & & & OF LAND & METHOD & & METHOD & DEPTH & DEPTH & & TYPE \\
\hline STA & TION & & & SURFACE & ALTITUDE & DATE OF & CONST - & DRILLED & OF WELL & CASING & OF \\
\hline NUM & BER & LAT I TUDE & LONGITUDE & (FEET) & DETERMINED & CONSTRUCTION & RUCTED & (FEET) & (FEET) & (IN) & FINISH \\
\hline NHW & 640 & 410927 & 0713643 & 160 & $M$ & $00-00-89$ & $\mathrm{H}$ & 63 & 63 & $\cdots$ & $\mathrm{s}$ \\
\hline NHW & 650 & 411304 & 0713350 & 60 & M & $07-16 \cdot 68$ & c & 134 & 133 & 6 & $\mathbf{s}$ \\
\hline NHW & 651 & 411257 & 0713404 & $1_{88}$ & M & $06-27 \cdot 77$ & c & 108 & 108 & 6 & $s$ \\
\hline NHW & 652 & 411240 & 0713407 & 80 & M & $08 \cdot 19 \cdot 71$ & C & 110 & 109 & 6 & $s$ \\
\hline NHW & 654 & 411234 & 0713410 & 62 & M & $06-26-75$ & c & 124 & 124 & 6 & $s$ \\
\hline & & & & & & -. & - & & & - & - \\
\hline NHW & 655 & 411239 & 0713406 & 86 & $M$ & $04-22-69$ & C & 110 & 110 & 6 & s \\
\hline NHW & 656 & 411232 & 0713410 & 55 & M & $05-26-83$ & A & 85 & 85 & 6 & $s$ \\
\hline NHW & 657 & 411228 & 0713404 & 53 & M & $08-31-76$ & C & 84 & 84 & 6 & s \\
\hline NHW & 658 & 411159 & 0713424 & 8 & M & $09 \cdot 27 \cdot 77$ & c & 32 & 32 & 6 & $s$ \\
\hline NHW & 659 & 411129 & 0713413 & 17 & M & $07-11-64$ & C & 65 & 55 & 6 & s \\
\hline NHW & 660 & 411104 & 0713412 & 14 & A & $06-26-84$ & A & 111 & 111 & 6 & s \\
\hline NHW & 661 & 411028 & 0713352 & 5 & M & $06-15 \cdot 83$ & $z$ & 96 & 96 & 6 & s \\
\hline NHW & 662 & 411034 & 0713407 & 29 & $M$ & $10-07-69$ & C & 58 & 57 & 6 & $s$ \\
\hline NHW & 664 & 411050 & 0713427 & 5.66 & $\mathbf{L}$ & $06 \cdot 05 \cdot 86$ & $z$ & 139 & 139 & 6 & $s$ \\
\hline NHW & 665 & 411048 & 0713446 & 20 & $M$ & $04-25-68$ & c & 53 & 52 & 6 & $\mathbf{S}$ \\
\hline NHW & 667 & 411101 & 0713418 & $1_{40}$ & $M$ & $07-17-84$ & $z$ & 117 & 117 & 6 & $s$ \\
\hline NHW & 668 & 411216 & 0713356 & 20 & $M$ & $10-15-68$ & C & 111 & 111 & $\cdots$ & $s$ \\
\hline NHW & 669 & 411024 & 0713401 & $1_{30}$ & $M$ & $08 \cdot 25 \cdot 79$ & C & 100 & 100 & 6 & $s$ \\
\hline NHW & 670 & 410940 & 0713638 & 70 & M & $08-11-88$ & H & 110 & 110 & 4 & s \\
\hline NHW & 671 & 410949 & 0713535 & 165 & $M$ & $\cdots$ & - & 188 & 186 & -. & - \\
\hline NHW & 672 & 411056 & 0713609 & 25 & M & $07-29-71$ & C & 50 & 50 & 6 & s \\
\hline NHW & 673 & 411202 & 0713425 & 15 & M & $06-13-79$ & A & 46 & 45 & 6 & s \\
\hline NHW & 674 & 411224 & 0713416 & 38 & M & $09-13-78$ & C & 64 & 64 & 6 & s \\
\hline NHW & 675 & 411155 & 0713353 & 30 & M & $08-00-87$ & A & 64 & 64 & 6 & s \\
\hline NHW & 676 & 410942 & 0713609 & 80 & $M$ & $09 \cdot 02 \cdot 80$ & A & 185 & 184 & 6 & $s$ \\
\hline & & & & & & $\cdots$ & $\cdot$ & & & $\cdots$ & $\cdot$ \\
\hline NHW & 677 & 410945 & 0713608 & 50 & $M$ & $08-21-80$ & A & 124 & 124 & 6 & $s$ \\
\hline & & & & & & $\cdots$ & - & & & $\cdots$ & $\cdot$ \\
\hline NHW & 678 & 410939 & 0713604 & 95 & $M$ & $05-09-74$ & c & 131 & 131 & 6 & $s$ \\
\hline NHW & 679 & 410934 & 0713550 & 125 & $M$ & $09-26-78$ & C & 195 & 195 & 6 & s \\
\hline & & & & & & $\cdots$ & - & & & $\cdots$ & $\cdot$ \\
\hline NHW & 680 & 411029 & 0713603 & 65 & $M$ & $06-01-71$ & C & 106 & 106 & 6 & s \\
\hline NHW & 682 & 410952 & 0713507 & 159 & A & $05-08-80$ & A & 237 & 237 & 6 & $s$ \\
\hline NHW & 684 & 410858 & 0713458 & 110 & $M$ & $06-24-69$ & c & 168 & 168 & 6 & $\mathbf{s}$ \\
\hline NHW & 685 & 411158 & 0713403 & 38 & $M$ & $05-20 \cdot 75$ & C & 69 & 68 & $\cdots$ & $s$ \\
\hline NHW & 686 & 410912 & 0713442 & 116 & A & $09-16-69$ & c & 184 & 184 & 6 & $s$ \\
\hline & & & & & & $\cdots$ & $\cdot$ & & & $\cdots$ & $\cdot$ \\
\hline NHW & 687 & 411025 & 0713403 & 19.85 & $\mathbf{L}$ & $05-07-82$ & A & 135 & 135 & 6 & $\mathrm{~s}$ \\
\hline & & & & & & -. & - & & & .. & - \\
\hline
\end{tabular}


Table 1.--Description of selected wells, springs, and test borings--Continued

$$
\text { [.- or -, no data available; } 00 \text { in date columns indicates unknown] }
$$

\begin{tabular}{|c|c|c|c|c|c|c|c|c|c|c|c|}
\hline \multicolumn{2}{|c|}{$\begin{array}{l}\text { STATION } \\
\text { NUMBER }\end{array}$} & \multirow{2}{*}{$\begin{array}{c}\text { TOP OF } \\
\text { OPEN } \\
\text { INTERVAL } \\
\text { (FEET) }\end{array}$} & $\begin{array}{l}\text { BOTTOM OF } \\
\text { OPEN } \\
\text { INTERVAL } \\
\text { (FEET) }\end{array}$ & $\begin{array}{l}\text { WATER } \\
\text { LEVEL } \\
\text { (FEET) }\end{array}$ & $\begin{array}{l}\text { DATE } \\
\text { WATER } \\
\text { LEVEL } \\
\text { MEASURED }\end{array}$ & $\begin{array}{l}\text { METHOD } \\
\text { WATER } \\
\text { LEVEL } \\
\text { MEASURED }\end{array}$ & $\begin{array}{l}\text { PRIMARY } \\
\text { USE } \\
\text { OF } \\
\text { SITE }\end{array}$ & $\begin{array}{l}\text { PRIMARY } \\
\text { USE } \\
\text { OF } \\
\text { WATER }\end{array}$ & $\begin{array}{l}\text { DISCHARGE } \\
\text { (GPM) }\end{array}$ & $\begin{array}{l}\text { DRAWDOWN } \\
\text { (FEET) }\end{array}$ & \multirow{2}{*}{$\begin{array}{c}\text { TYPE } \\
\text { OF LOG } \\
\text { AVAILABLE } \\
\text { D }\end{array}$} \\
\hline NHW & 640 & & 63 & 56 & $00 \cdot 00 \cdot 89$ & $\mathbf{R}$ & $\mathbf{W}$ & H & 5 & $\cdots$ & \\
\hline NHW & 650 & 130 & 133 & 60 & $07-16-68$ & $\mathbf{R}$ & $w$ & H & 8 & .. & $D$ \\
\hline NHW & 651 & 106 & 108 & 86 & $06-27-77$ & $R$ & $W$ & H & 30 & $-\cdot$ & $D$ \\
\hline NHW & 652 & 106 & 109 & 78 & $08-19-71$ & $\mathbf{R}$ & $W$ & H & 9 & $\cdots$ & $D$ \\
\hline \multirow[t]{2}{*}{ NHW } & 654 & 121 & 124 & 63 & $06-26-75$ & $\mathbf{R}$ & $W$ & H & 7 & $\cdots$ & D \\
\hline & & $\cdots$ & -. & & & & & & 5 & 17 & $\cdot$ \\
\hline NHW & 655 & 106 & 110 & 88 & $04-22-69$ & $\mathbf{R}$ & $W$ & $H$ & 9 & $\cdots$ & D \\
\hline NHW & 656 & 82 & 85 & 55 & $05-26 \cdot 83$ & $\mathbf{R}$ & $W$ & H & 65 & $\cdots$ & $D$ \\
\hline NHW & 657 & 80 & 84 & 53 & $08-31-76$ & $\mathbf{R}$ & $W$ & H & 25 & $\cdots$ & D \\
\hline NHW & 658 & 28 & 32 & 6 & $09 \cdot 27 \cdot 77$ & $\mathbf{R}$ & $W$ & H & 3 & $\cdots$ & D \\
\hline NHW & 659 & 51 & 55 & 17 & $07 \cdot 11 \cdot 64$ & $\mathbf{R}$ & $w$ & H & 10 & $\cdots$ & $\cdot$ \\
\hline NHW & 660 & 108 & 111 & 15 & $06 \cdot 26 \cdot 84$ & $\mathbf{R}$ & $w$ & $\mathrm{H}$ & 6 & 30 & $D$ \\
\hline NHW & 661 & 93 & 96 & 4 & $06 \cdot 15 \cdot 83$ & $\mathbf{R}$ & $w$ & H & 3 & .. & D \\
\hline NHW & 662 & 53 & 57 & 28 & $10 \cdot 07 \cdot 69$ & $\mathbf{R}$ & $W$ & $\mathrm{H}$ & 38 & $\cdots$ & $D$ \\
\hline NHW & 664 & $\cdots$ & $\cdots$ & 3 & $06-05-86$ & $\mathbf{R}$ & $W$ & $c$ & 35 & $\cdots$ & $D$ \\
\hline NHW & 665 & 48 & 52 & 12 & $04-25-68$ & $\mathbf{R}$ & $w$ & 2 & 10 & -. & D \\
\hline NHW & 667 & 114 & 117 & 40 & $07 \cdot 17 \cdot 84$ & $\mathbf{R}$ & $W$ & $H$ & 6 & $\cdots$ & D \\
\hline NHW & 668 & 108 & 111 & 31 & $09 \cdot 24 \cdot 70$ & $R$ & $W$ & $H$ & 18 & 29 & D \\
\hline NHW & 669 & 96 & 100 & 30 & $08 \cdot 25 \cdot 79$ & $\mathbf{R}$ & $W$ & C & 7 & 60 & D \\
\hline NHW & 670 & 105 & 110 & 72.2 & $08-12-88$ & $\mathbf{s}$ & $W$ & $H$ & 60 & $\cdots$ & $D$ \\
\hline NHW & 671 & $\cdots$ & $\cdots$ & 83 & $08 \cdot 18 \cdot 88$ & $\mathbf{R}$ & $w$ & $H$ & $\cdots$ & .. & - \\
\hline NHW & 672 & 48 & 50 & 24 & $07-29-71$ & $\mathbf{R}$ & $w$ & H & 25 & 4 & $\cdot$ \\
\hline NHW & 673 & 42.3 & 45 & 15 & $06-13-79$ & $\mathbf{R}$ & $w$ & H & 50 & 30 & D \\
\hline NHW & 674 & 60.5 & 64 & 38 & $09-13-78$ & $\mathbf{R}$ & $W$ & H & 50 & $\cdots$ & $D$ \\
\hline NHW & 675 & 60.7 & 64 & $\cdots$ & $\cdots$ & $\cdot$ & $W$ & H & 80 & $\cdots$ & D \\
\hline \multirow[t]{2}{*}{ NHW } & 676 & 181 & 184 & 80 & $09 \cdot 02 \cdot 80$ & $\mathbf{R}$ & $w$ & H & 50 & $\cdots$ & D \\
\hline & & $\cdots$ & $\cdots$ & & & & & & 11 & 10 & $\cdot$ \\
\hline \multirow[t]{2}{*}{ NHW } & 677 & 121 & 124 & 45 & $08-21-80$ & $\mathbf{R}$ & $W$ & H & 50 & -. & $D$ \\
\hline & & $\cdots$ & $\cdots$ & & & & & & 12 & 40 & $\cdot$ \\
\hline NHW & 678 & 128 & 131 & 75 & $05 \cdot 09-74$ & $\mathbf{R}$ & $W$ & H & 15 & 15 & $D$ \\
\hline \multirow[t]{2}{*}{ NHW } & 679 & 192 & 195 & 120 & $09-26-78$ & $R$ & $w$ & H & 30 & 75 & $D$ \\
\hline & & $\cdots$ & - & & & & & & 8 & 10 & $\cdot$ \\
\hline NHW & 680 & 103 & 106 & 63 & $06-01-71$ & $\mathbf{R}$ & $W$ & H & 7 & 7 & D \\
\hline NHW & 682 & 234 & 237 & 138 & $05-08 \cdot 80$ & $\mathbf{R}$ & $w$ & H & 12 & 99 & D \\
\hline NHW & 684 & 167 & 168 & 70 & $06 \cdot 16 \cdot 70$ & $\mathbf{R}$ & $w$ & $H$ & 10 & 40 & $D$ \\
\hline NHW & 685 & 64 & 68 & 38 & $05 \cdot 20 \cdot 75$ & $\mathbf{R}$ & $w$ & $H$ & 45 & $\cdots$ & $D$ \\
\hline \multirow[t]{2}{*}{ NHW } & 686 & 181 & 184 & 115 & $09-16-69$ & $\mathbf{R}$ & $w$ & $H$ & 18 & $\cdots$ & $D$ \\
\hline & & - & - & & & & & & 8 & 15 & - \\
\hline \multirow[t]{2}{*}{ NHW } & 687 & 132 & 135 & 17 & $05 \cdot 07 \cdot 82$ & $\mathbf{R}$ & $W$ & $H$ & 50 & $\cdots$ & D \\
\hline & & $\cdots$ & $\cdots$ & & & & & & 5 & 5 & $\cdot$ \\
\hline
\end{tabular}


Table 1.--Description of selected wells, springs, and test borings--Continued

[-- or -, no data available; 00 in date columns indicates date unknown]

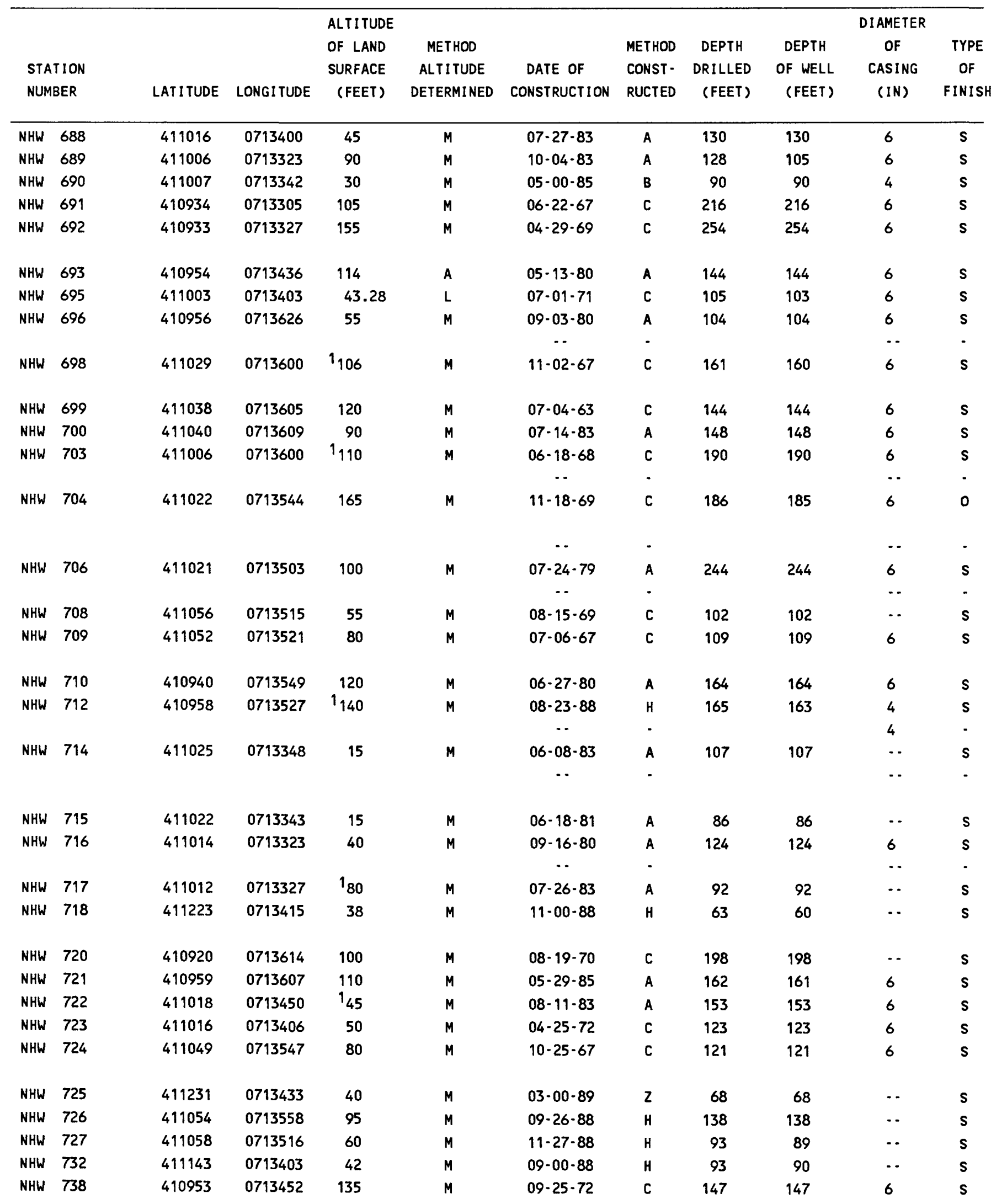


Table 1.--Description of selected wells, springs, and test borings--Continued

[-- or -, no data available; 00 in date columns indicates unknown]

\begin{tabular}{|c|c|c|c|c|c|c|c|c|c|c|c|}
\hline \multicolumn{2}{|c|}{$\begin{array}{l}\text { STATION } \\
\text { NUMBER }\end{array}$} & $\begin{array}{l}\text { TOP OF } \\
\text { OPEN } \\
\text { INTERVAL } \\
\text { (FEET) }\end{array}$ & $\begin{array}{l}\text { BOTTOM OF } \\
\text { OPEN } \\
\text { INTERVAL } \\
\text { (FEET) }\end{array}$ & $\begin{array}{l}\text { WATER } \\
\text { LEVEL } \\
\text { (FEET) }\end{array}$ & $\begin{array}{l}\text { DATE } \\
\text { WATER } \\
\text { LEVEL } \\
\text { MEASURED }\end{array}$ & $\begin{array}{l}\text { METHOD } \\
\text { WATER } \\
\text { LEVEL } \\
\text { MEASURED }\end{array}$ & $\begin{array}{c}\text { PRIMARY } \\
\text { USE } \\
\text { OF } \\
\text { SITE }\end{array}$ & $\begin{array}{l}\text { PRIMARY } \\
\text { USE } \\
\text { OF } \\
\text { WATER }\end{array}$ & $\begin{array}{c}\text { DISCHARGE } \\
\text { (GPM) }\end{array}$ & $\begin{array}{l}\text { DRAWDOWN } \\
\text { (FEET) }\end{array}$ & $\begin{array}{c}\text { TYPE } \\
\text { OF LOG } \\
\text { AVAILABLE }\end{array}$ \\
\hline NHW & 688 & 127 & 130 & $\cdots$ & $\therefore$ & - & $w$ & $\mathrm{H}$ & 100 & $\cdots$ & $D$ \\
\hline NHW & 689 & 102 & 105 & 65 & $10-04-83$ & $R$ & $W$ & $H$ & 50 & .. & D \\
\hline NHW & 690 & $\cdots$ & $\cdots$ & -. & $\cdots$ & - & $W$ & $H$ & 60 & $\cdots$ & - \\
\hline NHW & 691 & 213 & 216 & 100 & $06-22 \cdot 67$ & $\mathbf{R}$ & $W$ & H & 35 & 7 & D \\
\hline NHW & 692 & 252 & 254 & 154 & $04-29-69$ & $\mathbf{R}$ & $W$ & $H$ & 20 & 0 & D \\
\hline NHW & 693 & 141 & 144 & 80 & $05-13-80$ & $\mathbf{R}$ & $W$ & $H$ & 12 & $\cdots$ & $D$ \\
\hline NHW & 695 & 100 & 103 & 22 & $07-01-71$ & $\mathbf{R}$ & $W$ & $H$ & 18 & 13 & D \\
\hline \multirow[t]{2}{*}{ NHW } & 696 & 100 & 104 & 40 & $09-03-80$ & $\mathbf{R}$ & $W$ & H & 50 & - & D \\
\hline & & $\cdots$ & $\cdots$ & & & & & & 10 & 10 & - \\
\hline NHW & 698 & 158 & 160 & 106 & $11-02 \cdot 67$ & $\mathbf{R}$ & $W$ & $H$ & 12 & 24 & $D$ \\
\hline NHW & 699 & 140 & 144 & 120 & $07-04 \cdot 63$ & $\mathbf{R}$ & $w$ & $H$ & 7 & 7 & D \\
\hline NHW & 700 & 145 & 148 & -. & $\cdots$ & $\cdot$ & $w$ & H & 50 & -. & D \\
\hline \multirow[t]{2}{*}{ NHW } & 703 & 187 & 190 & 90 & $06 \cdot 18-68$ & $\mathbf{R}$ & $W$ & $H$ & 7 & 100 & D \\
\hline & & $\cdots$ & $\cdots$ & & & & & & 4 & 50 & $\cdot$ \\
\hline \multirow[t]{2}{*}{ NHW } & 704 & $\cdots$ & $\cdots$ & 152 & $11-18-69$ & $\mathbf{R}$ & $W$ & $H$ & 15 & 33 & D \\
\hline & & $\cdots$ & - & & & & & & 7 & 18 & - \\
\hline \multirow[t]{2}{*}{ NHW } & 706 & 241 & 244 & 91 & $07-24-79$ & $\mathbf{R}$ & $w$ & H & 60 & 153 & D \\
\hline & & $\cdots$ & $\cdots$ & & & & & & 15 & 49 & $\cdot$ \\
\hline NHW & 708 & 99 & 102 & 72 & $08 \cdot 15 \cdot 69$ & $\mathbf{R}$ & W & $H$ & 35 & $\cdots$ & D \\
\hline NHW & 709 & 105 & 109 & 78 & $07-06-67$ & $\mathbf{R}$ & $W$ & H & 35 & 2 & $D$ \\
\hline NHW & 710 & 161 & 164 & 75 & $06-27-80$ & $\mathbf{R}$ & $W$ & H & 100 & $\cdots$ & D \\
\hline \multirow[t]{2}{*}{ NHW } & 712 & 138 & 143 & 141 & $08-24-88$ & S & $W$ & $H$ & - & $\cdots$ & D \\
\hline & & $\cdots$ & $\cdots$ & & & & & & $\cdots$ & $\cdots$ & - \\
\hline \multirow[t]{2}{*}{ NHW } & 714 & 104 & 107 & 14 & $06-08-83$ & $\mathbf{R}$ & $W$ & c & 50 & -. & D \\
\hline & & $\cdots$ & $\cdots$ & & & & & & 25 & 26 & - \\
\hline NHW & 715 & 83 & 86 & 16 & $06-18-81$ & $R$ & u & $u$ & 100 & $\cdots$ & D \\
\hline \multirow[t]{2}{*}{ NHW } & 716 & 121 & 124 & 38 & $09 \cdot 16 \cdot 80$ & $\mathbf{R}$ & $W$ & $\mathbf{T}$ & 100 & -. & D \\
\hline & & $\cdots$ & $\cdots$ & & & & & & 158 & $\cdots$ & $\cdot$ \\
\hline NHW & 717 & 89 & 92 & 8 & $07-26-83$ & $\mathbf{R}$ & $W$ & $H$ & 40 & $\cdots$ & D \\
\hline NHW & 718 & 55 & 60 & 40 & $11-00-88$ & $\mathbf{R}$ & $W$ & $H$ & - & $\cdots$ & $D$ \\
\hline NHW & 720 & 194 & 198 & 98 & $08-19-70$ & $\mathbf{R}$ & $W$ & H & 30 & 3 & D \\
\hline NHW & 721 & 158 & 161 & 80 & $05-29-85$ & $\mathbf{R}$ & $W$ & $H$ & 50 & $\cdots$ & D \\
\hline NHW & 722 & 150 & 153 & 75 & $08-11-83$ & $\mathbf{R}$ & $W$ & $H$ & 50 & $\cdots$ & D \\
\hline NHW & 723 & 120 & 123 & 50 & $04-25-72$ & $\mathbf{R}$ & $W$ & $H$ & 35 & 5 & D \\
\hline NHW & 724 & 118 & 121 & 89 & $10-25-67$ & $\mathbf{R}$ & $W$ & $H$ & 35 & 7 & $D$ \\
\hline NHW & 725 & 58 & 68 & 35 & $03 \cdot 00-89$ & $R$ & $W$ & $P$ & 75 & $-\cdot$ & D \\
\hline NHW & 726 & $-\cdot$ & $\cdots$ & 88 & $09-26-88$ & $\mathbf{R}$ & $W$ & $\mathrm{H}$ & 60 & $\cdots$ & D \\
\hline NHW & 727 & $\cdots$ & $\cdots$ & 77 & $11-27-88$ & $R$ & $W$ & $H$ & 10 & $\cdots$ & D \\
\hline NHW & 732 & 65 & 70 & 33 & $09-00-88$ & $R$ & $W$ & $H$ & -. & $\cdots$ & $D$ \\
\hline NHW & 738 & 144 & 147 & 95 & $09 \cdot 25 \cdot 72$ & $\mathbf{R}$ & $W$ & $H$ & 10 & $\cdots$ & D \\
\hline
\end{tabular}


Table 1.--Description of selected wells, springs, and test borings--Continued

$$
\text { [-- or -, no data available; } 00 \text { in date columns indicates date unknown] }
$$

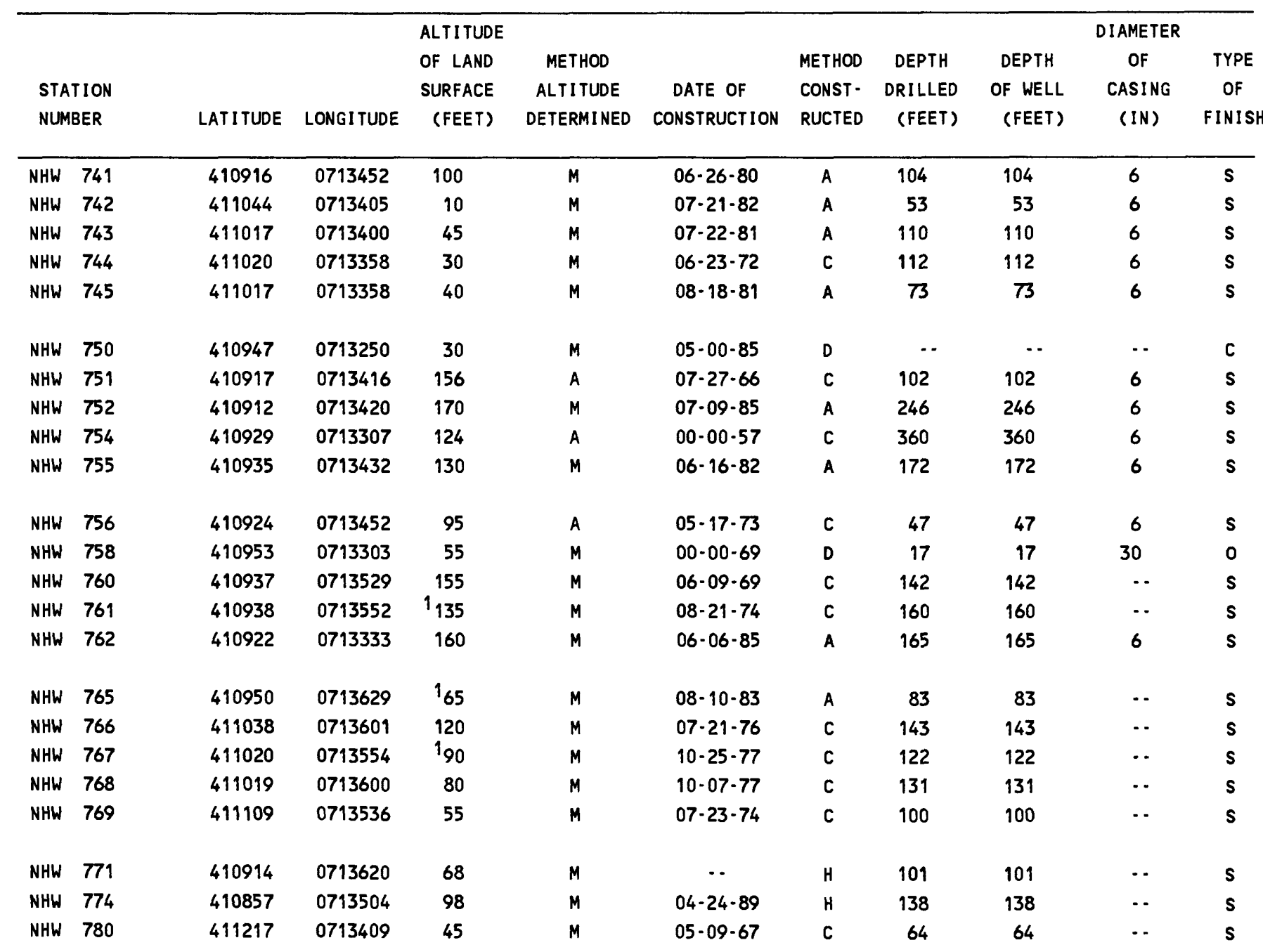


Table 1.-Description of selected wells, springs, and test borings--Continued

[-. or -, no data available; 00 in date columns indicates unknown]

\begin{tabular}{|c|c|c|c|c|c|c|c|c|c|c|c|}
\hline \multicolumn{2}{|c|}{$\begin{array}{l}\text { STATION } \\
\text { NUMBER }\end{array}$} & $\begin{array}{l}\text { TOP OF } \\
\text { OPEN } \\
\text { INTERVAL } \\
\text { (FEET) }\end{array}$ & $\begin{array}{l}\text { BOTTOM OF } \\
\text { OPEN } \\
\text { INTERVAL } \\
\text { (FEET) }\end{array}$ & $\begin{array}{l}\text { WATER } \\
\text { LEVEL } \\
\text { (FEET) }\end{array}$ & $\begin{array}{l}\text { DATE } \\
\text { WATER } \\
\text { LEVEL } \\
\text { MEASURED }\end{array}$ & $\begin{array}{l}\text { METHOD } \\
\text { WATER } \\
\text { LEVEL } \\
\text { MEASURED }\end{array}$ & $\begin{array}{l}\text { PRIMARY } \\
\text { USE } \\
\text { OF } \\
\text { SITE }\end{array}$ & $\begin{array}{l}\text { PRIMARY } \\
\text { USE } \\
\text { OF } \\
\text { WATER }\end{array}$ & $\begin{array}{l}\text { DISCHARGE } \\
\text { (GPM) }\end{array}$ & $\begin{array}{l}\text { DRAWDOWN } \\
\text { (FEET) }\end{array}$ & $\begin{array}{c}\text { TYPE } \\
\text { OF LOG } \\
\text { AVAILABLE }\end{array}$ \\
\hline NHW & 741 & 101 & 104 & 62 & $06-26-80$ & $\mathrm{R}$ & $w$ & H & 45 & $\cdots$ & D \\
\hline NHW & 742 & $\cdots$ & $\cdots$ & 4 & $07-21-82$ & $R$ & $w$ & H & 30 & $\cdots$ & D \\
\hline NHW & 743 & 107 & 110 & 50 & $07-22 \cdot 81$ & $R$ & $w$ & H & 10 & 50 & D \\
\hline NHW & 744 & 108 & 112 & 26 & $06-23 \cdot 72$ & $R$ & W & H & 35 & 9 & D \\
\hline NHW & 745 & 70 & 73 & 20 & $08-18-81$ & $\mathbf{R}$ & $w$ & H & 10 & $\cdots$ & D \\
\hline NHW & 750 & $\cdots$ & $\cdots$ & 9.86 & $06-07-89$ & s & W & H & .. & .. & - \\
\hline NHW & 751 & 98 & 102 & -. & -. & - & W & H & 20 & -. & D \\
\hline NHW & 752 & 243 & 246 & 145 & $07-09-85$ & $R$ & $w$ & H & 50 & .. & D \\
\hline NHW & 754 & 350 & 360 & .. & .. & - & $w$ & H & 200 & .. & - \\
\hline NHW & 755 & $\cdots$ & $-\cdot$ & 56 & $06-16-82$ & $R$ & $w$ & $H$ & 3 & -. & - \\
\hline NHW & 756 & 44 & 47 & 8 & $05-17-73$ & $\mathbf{R}$ & $w$ & $H$ & 15 & .. & D \\
\hline NHW & 758 & -. & $\cdots$ & 0.81 & $06-15-89$ & s & $w$ & $H$ & 2 & .. & - \\
\hline NHW & 760 & 139 & 142 & 90 & $06.09 \cdot 69$ & $R$ & $w$ & H & 25 & 10 & D \\
\hline NHW & 761 & 157 & 160 & 90 & $08-21 \cdot 74$ & $R$ & $w$ & $H$ & 35 & 30 & D \\
\hline NHW & 762 & 162 & 165 & 135 & $06-06-85$ & $\mathrm{R}$ & $w$ & H & 6 & .. & D \\
\hline NHW & 765 & 80 & 83 & 30 & $08-10-83$ & $R$ & $w$ & H & 100 & .. & D \\
\hline NHW & 766 & 140 & 143 & 114 & $07-21 \cdot 76$ & $R$ & w & H & 30 & .. & D \\
\hline NHW & 767 & 118 & 122 & 58 & $10 \cdot 25 \cdot 77$ & $\mathbf{R}$ & W & H & 8 & 32 & D \\
\hline NHW & 768 & 128 & 131 & 76 & $10 \cdot 07 \cdot 77$ & $\mathbf{R}$ & W & $H$ & 8 & 34 & D \\
\hline NHW & 769 & 97 & 100 & 45 & $07 \cdot 23 \cdot 74$ & $R$ & $w$ & H & 18 & 25 & D \\
\hline NHW & 771 & 96 & 101 & .. & .. & . & $w$ & H & .. & .. & D \\
\hline NHW & 774 & 133 & 138 & 98 & $04-24-89$ & R & $W$ & $H$ & .. & $\cdots$ & D \\
\hline NHW & 780 & 61 & 64 & 38 & $06-09-67$ & $R$ & $w$ & H & 40 & 4 & D \\
\hline
\end{tabular}

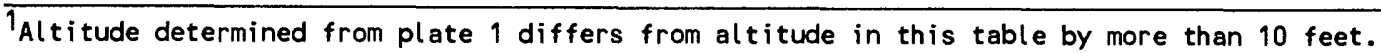


Table 2.--Lithologic logs of selected wells and test borings

[NHB, boring; NHW, we11]

\begin{tabular}{l} 
Depth below $\begin{array}{l}\text { Description of } \\
\text { land surface the sediment } \\
\text { (in feet) }\end{array}$ \\
\hline
\end{tabular}

\begin{tabular}{|c|c|}
\hline $0-37$ & $\begin{array}{l}\text { NHW } 1 \\
\text { sand and clay }\end{array}$ \\
\hline $0-34$ & $\begin{array}{l}\text { NHW } 2 \\
\text { sand and clay }\end{array}$ \\
\hline $0-37$ & $\begin{array}{l}\text { NHW } 3 \\
\text { clay and sand }\end{array}$ \\
\hline $0-63$ & $\begin{array}{l}\text { NHW } 11 \\
\text { grave } 1\end{array}$ \\
\hline $\begin{array}{l}0-52 \\
52\end{array}$ & $\begin{array}{l}\text { NHW } 12 \\
\text { sand } \\
\text { clay, blue }\end{array}$ \\
\hline $0-89.8$ & $\begin{array}{l}\text { NHW } 15 \\
\text { clay }\end{array}$ \\
\hline $\begin{array}{r}1-25 \\
25-38 \\
38-43 \\
43-75 \\
75-145 \\
145-155\end{array}$ & $\begin{array}{l}\text { NHW } 31 \\
\text { sand and gravel } \\
\text { till } \\
\text { boulders } \\
\text { till } \\
\text { clay, white-gray } \\
\text { sand and gravel }\end{array}$ \\
\hline $\begin{array}{r}0-10 \\
10-38 \\
38-78 \\
78-91 \\
91-118 \\
118-124 \\
124-132 \\
132-160\end{array}$ & $\begin{array}{l}\text { NHW } 33 \\
\text { gravel and clay } \\
\text { clay } \\
\text { gravel } \\
\text { clay, white } \\
\text { gravel and sand } \\
\text { clay } \\
\text { grave1, coarse } \\
\text { clay }\end{array}$ \\
\hline $\begin{array}{l}0-11 \\
11-15 \\
15-26 \\
26-57 \\
57-59 \\
59-68 \\
68-75\end{array}$ & $\begin{array}{l}\text { NHW } 35 \\
\text { sand, fine, and silt } \\
\text { sand and silt } \\
\text { sand, gravelly } \\
\text { clay } \\
\text { sand and gravel } \\
\text { clay } \\
\text { sand gravel }\end{array}$ \\
\hline
\end{tabular}

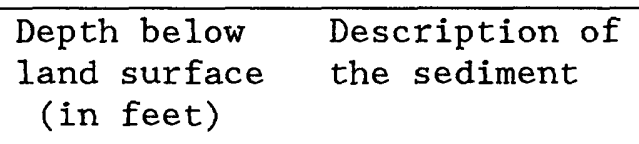

\begin{tabular}{cl}
\hline Depth below & Description of \\
land surface & the sediment \\
(in feet) & \\
\hline
\end{tabular}

$\begin{aligned} 0-41 & \text { sand, clay, sandy clay } \\ 41-49 & \text { sand and grave1 } \\ 0-10 & \text { NHW } 38 \\ 10-24 & \text { sand } \\ 24-30.2 & \text { gray } \\ & \text { NHW } 39 \\ 0-30 & \text { sand } \\ 30-150 & \text { till and clay } \\ 150-155 & \text { grave1 } \\ & \text { NHW 40 } \\ 0-52 & \text { sand and clay } \\ 52-61 & \text { grave1 } \\ & \text { NHW 41 } \\ 0-15 & \text { sand and gravel } \\ 15-59 & \text { clay and hardpan } \\ 59-69 & \text { grave1 (no water of any } \\ 69-101 & \text { amount) } \\ 101-109 & \text { gray and hardpan } \\ & \text { gravel; water }\end{aligned}$

$101-109$

$0-86$

$86-96$

$0-101$

$101-105$

$0-133$

$133-135$

135-139

$0-100$

100

$100-138$

$138-143$

\section{NHW 37}

sand, clay, sandy clay

NHW 38

sand

clay

grave 1

NHW 39

till and clay

NHW 42

unknown

sand and gravel

NHW 43

unknown

grave1

NHW 44

unknown

grave1

unknown

NHW 45

unknown

thin layer of gravel

till

sand and grave1 
Table 2.--Lithologic logs of selected wells and test borings--Continued

\begin{tabular}{ll}
\hline Depth below & Description of \\
land surface & the sediment \\
(in feet) & \\
\hline
\end{tabular}

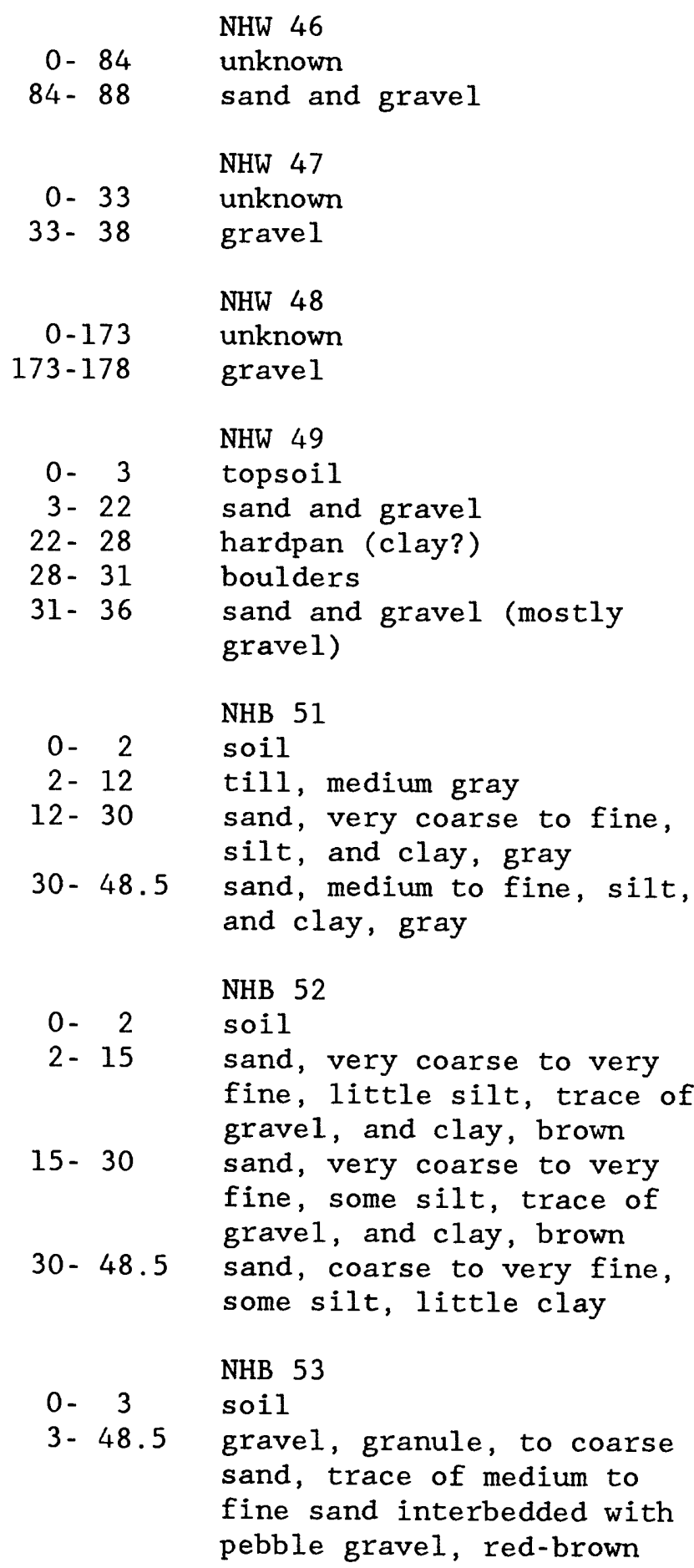

\begin{tabular}{cl}
\hline $\begin{array}{c}\text { Depth below } \\
\text { land surface } \\
\text { (in feet) }\end{array}$ & $\begin{array}{l}\text { Description of } \\
\text { the sediment }\end{array}$ \\
\hline
\end{tabular}

$\begin{array}{ll}0- & 2 \\ 2- & 7 \\ 7-\quad 8.5\end{array}$

NHB 54

8.5- $32 \quad$ gravel, gray 2 pebble, very coarse

to very fine sand, silt, trace of clay, gray (till)

32- 37 gravel, granule, well

rounded, little silt, traces

of pebble gravel, very

coarse to fine sand, gray

(washed till)

37- 38.5 gravel, granule, to very coarse sand, trace of silt, gray-brown (outwash)

NHB 55

\begin{tabular}{|c|c|}
\hline $0-$ & soil \\
\hline $1-$ & $\begin{array}{l}\text { sand, very fine, to silt, } \\
\text { trace of clay, white to buff }\end{array}$ \\
\hline $6-7$ & $\begin{array}{l}\text { sand, very fine, to silt, } \\
\text { trace of fine sand, medium } \\
\text { sand, coarse sand, very } \\
\text { coarse sand, white, buff, } \\
\text { gray }\end{array}$ \\
\hline $7-9$ & $\begin{array}{l}\text { silt, very fine sand and } \\
\text { clay, trace of coarse sand } \\
\text { and very coarse sand, white } \\
\text { and gray }\end{array}$ \\
\hline $9-28$ & clay, some silt, white \\
\hline $3-32$ & $\begin{array}{l}\text { silt, some clay and medium } \\
\text { sand, little fine sand, } \\
\text { trace coarse sand, white to } \\
\text { light gray }\end{array}$ \\
\hline-38.5 & $\begin{array}{l}\text { clay, silt and fine sand, } \\
\text { white; becomes pink near } \\
\text { bottom }\end{array}$ \\
\hline
\end{tabular}


Table 2.--Lithologic logs of selected wells and test borings--Continued

\begin{tabular}{ll}
\hline Depth below & Description of \\
land surface & the sediment \\
(in feet) & \\
\hline
\end{tabular}

\section{NHB 56}

\section{$0-3$}

topsoil

$3-5$

gravel, pebble to granule, little fine sand, very fine sand and silt, trace medium sand, coarse sand, very

coarse sand, gray (till)

5- 7 sand, coarse and medium, little fine sand, very fine sand, trace silt and granule grave1, brown

7- 10 gravel, pebble to granule, little fine sand, very fine sand and silt, trace medium sand, coarse sand, very coarse sand, gray (till)

10- 13 sand, very coarse to very fine, little granule gravel, trace pebble gravel, brown

13- 14 gravel, pebble to granule, brown

14- 17 sand, medium, some fine sand, little very fine sand and silt, brown; interbedded with gravel

17- 32 sand, medium, some fine sand, little very fine sand and silt, brown

32-43.5 sand, medium and fine, little very fine sand and silt, brown

$\begin{array}{cl}\text { Depth below } & \text { Description of } \\ \text { land surface } & \text { the sediment } \\ \text { (in feet) } & \end{array}$

$\begin{array}{lll}0- & 1 \\ 1- & 5\end{array}$

NHB 57

5- 17 silt and very fine sand,

sand, very coarse to very

fine, little granule and pebble gravel, and silt, little clay and fine sand, gray

17- 27 sand, medium to very fine, some silt, little coarse and very coarse sand, brown

27- 60 gravel, granule to coarse sand, little medium to very fine sand, little pebble gravel, sand, brown

60- 63.5 sand, very coarse to coarse, little medium to very fine sand, brown

NHB 58

$0-5$ sand, very coarse to very fine, trace of pebble gravel, granule gravel, silt, brown

5- 6 sand, fine and very fine, some silt, gray

6- 20 sand, fine and very fine, some silt, little granule grave1 and very coarse sand, brown

20-50 silt and clay, little very fine sand, trace of coarse sand, medium sand, fine sand, gray

50- 55 grave1, granule, some very coarse sand, little coarse sand, medium sand, fine sand, very fine sand, silt and clay; gray

55- 63.5 grave1, pebble to granule, some very coarse sand, little coarse sand, trace medium sand, fine sand, very fine sand, silt and clay, brown (not till) 
Table 2.--Lithologic logs of selected wells and test borings--Continued

\begin{tabular}{ll}
\hline Depth below & Description of \\
land surface & the sediment \\
(in feet) & \\
\hline
\end{tabular}

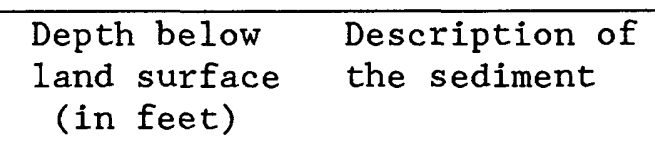

\section{NHB 59}

0 - 5 sand, very coarse to very fine, trace pebble gravel, granule gravel, silt, dark gray

5- 6 peat and silt, dark brown

6- 35 silt and very fine sand, little coarse sand, trace medium sand, fine sand, dark gray to dark brown

35- 40 sand, very coarse to medium, little granule gravel, silt, and very fine sand, trace fine sand, dark brown

40-63.5 sand, very coarse to medium, little granule gravel, trace fine sand, very fine sand, and silt, brown

NHB 60

0- 22 fine sand, little very fine sand, trace coarse sand and silt, micaceous, magnetite, dark gray

22- 40 very fine sand, some fine sand, little silt, micaceous, magnetite, dark gray to olive gray

40- 48 silt, some clay, trace very coarse sand to granule gravel, olive gray; also containing lenses of granule and pebble gravel
NHB 61

0- 11 sand, medium and fine, brown

11- 15 sand, medium and fine, little coarse sand and very fine sand, trace silt, dark brown

15- 25 sand, medium, little coarse sand, trace fine sand, very fine sand, silt, micaceous, gray

25-43.5 sand, fine and medium, little very fine sand and silt, trace coarse sand, micaceous, gray; possibly a few layers of gravel

NHW 62

0- 25

$25-40$

$40-80$

$80-140$

$140-175$

$175-310$

$310-316$

$0-50$

$50-60$

$60-80$

$0-50$

$50-60$

$60-65$

$0-20$

$20-40$

$40-60$

$60-85$

0- 1

$1-30$

$30-60$

$60-70$

$70-80$ sand, gravel

till

sand, gravel

till

clay, white

clay, red

sand, gravel

NHW 63

sand and gravel

till

sand and gravel

NHW 64

sand and gravel

till

sand and gravel

NHW 65

sand and gravel

hardpan

clay, gray and white

sand and gravel

NHW 66

topsoil

sand and gravel

hardpan

clay, gray-white

sand and gravel 
Table 2.--Lithologic logs of selected wells and test borings--Continued

\begin{tabular}{ll}
\hline Depth below & Description of \\
land surface & the sediment \\
(in feet) &
\end{tabular}

NHW 67

0 - 1 topsoil

$1-30$

$30-80$

$80-120$

$120-140$

$140-235$

235-240

$0-1$

$1-25$

$25-65$

$65-100$

$100-125$

$125-145$

$0-1$

$1-30$

$30-60$

$60-90$

$90-100$

$0-1$

$1-25$

$25-45$

$45-50$

$0-25$

25- 80

$80-104$

$0-10$

$10-40$

$40-75$

$75-105$

sand and gravel

hardpan

clay, grayish white

sand, fine

hardpan

sand and gravel

NHW 68

topsoil

sand and gravel

till

clay, grayish-white

sand, fine

sand and gravel

NHW 69

topsoil

sand, very fine, and cobbles

hardpan

clay, gray-white

sand and grave1

NHW 70

soil

sand and gravel

till

sand and gravel

NHW 71

sand and gravel

hardpan

sand and gravel

NHW 75

$0-17.2$ sand and gravel

NHW 76

$0-16.0$ sand, gravel

NHW 81

old cistern

sand and gravel

hardpan

sand and gravel

$\begin{array}{cl}\text { Depth below } & \text { Description of } \\ \text { land surface } & \text { the sediment } \\ \text { (in feet) } & \end{array}$

\begin{tabular}{|c|c|}
\hline $0-23.2$ & $\begin{array}{l}\text { NHW } 91 \\
\text { ti11, sandy }\end{array}$ \\
\hline $0-\quad 3.8$ & $\begin{array}{l}\text { NHW } 96 \\
\text { sand }\end{array}$ \\
\hline $\begin{aligned} 0 & -15 \\
15 & -19.7\end{aligned}$ & $\begin{array}{l}\text { NHW } 97 \\
\text { til1 } \\
\text { sand }\end{array}$ \\
\hline $0-\quad 5.3$ & $\begin{array}{l}\text { NHW } 101 \\
\text { silt and clay }\end{array}$ \\
\hline $\begin{array}{lc}0- & 5 \\
5- & 16.9\end{array}$ & $\begin{array}{l}\text { NHW } 102 \\
\text { till } \\
\text { silt and clay }\end{array}$ \\
\hline $0-12.9$ & $\begin{array}{l}\text { NHW } 108 \\
\text { sand and silt }\end{array}$ \\
\hline $0-15.2$ & $\begin{array}{l}\text { NHW } 119 \\
\text { till }\end{array}$ \\
\hline $0-15.5$ & $\begin{array}{l}\text { NSH } 120 \\
\text { ti11 }\end{array}$ \\
\hline $\begin{aligned} 0 & -10 \\
10- & 12 \\
12- & 18.6\end{aligned}$ & $\begin{array}{l}\text { NHW } 148 \\
\text { sand and gravel } \\
\text { silt } \\
\text { sand and grave1 }\end{array}$ \\
\hline $0-26.2$ & $\begin{array}{l}\text { NHW } 152 \\
\text { sand and clay }\end{array}$ \\
\hline $\begin{array}{rr}0- & 8 \\
8- & 10 \\
10- & 21\end{array}$ & $\begin{array}{l}\text { NHW } 154 \\
\text { till } \\
\text { clay } \\
\text { clay and sand, interbedded }\end{array}$ \\
\hline $\begin{array}{ll}0- & 5 \\
5- & 7 \\
7- & 9\end{array}$ & $\begin{array}{l}\text { NHW } 162 \\
\text { sand, gravel } \\
\text { clay } \\
\text { sand, gravel }\end{array}$ \\
\hline $0-12.7$ & $\begin{array}{l}\text { NHW } 163 \\
\text { silt, sand }\end{array}$ \\
\hline
\end{tabular}


Table 2.--Lithologic logs of selected wells and test borings--Continued

\begin{tabular}{ll}
\hline Depth below & Description of \\
land surface & the sediment \\
(in feet) & \\
\hline
\end{tabular}

\section{NHW 172}

\section{$0-7.2$ sand}

NHW 175

$0-4$ sand and grave1

4- 12 til1

12- 13.0 sand and gravel

NHW 194

$0-10.5$ sand

NHW 214

$\begin{aligned} 0-20 & \text { till } \\ 20-21 & \text { sand and gravel }\end{aligned}$

NHW 220
silt

NHW 227

0 - 38 unknown

$38-75$

$75-110$

$110-151$

$0-18$

$18-21$

$21-23$

0- 10

$10-42$

$42-56$

$56-62$

$62-73$

$73-78$ sand and gravel

hardpan

sand and gravel

NHW 229

clay, predominantly

sand and grave1

sand

NHW 237

sand, loamy

sand and gravel, brown

grave1, clayey

hardpan

sand, brown and white

sand and gravel,

clean

\begin{tabular}{cl}
\hline Depth below & Description of \\
land surface & the sediment \\
(in feet) & \\
\hline
\end{tabular}

$\begin{array}{rr}0- & 1 \\ 1- & 3 \\ 3- & 11 \\ 11- & 36 \\ 36-68 \\ 68-82 \\ 82-88 \\ 88-103\end{array}$

$1-7$

7- 11

$11-27$

$27-36$

$36-42$

$42-49$

$49-56$

$56-66$

$66-78$

$78-95$

$95-104$

$104-127$

$0-10$

$10-15$

$15-32$

$32-36$

$36-44$

$44-52$

$52-64$

$64-78$

$78-88$

$88-98$

$98-102$

$102-106$
NHW 238

soil

sand and gravel, loamy

gravel, coarse, and cobbles

sand, clayey

sand, medium, brown

clay, blue-white

sand, beach, brown

sand and grave1, clean

NHW 239

sand, loamy, clayey

gravel, clayey

sand, fine, brownish gray

clay, gray

clay, brown

grave1, clayey

gravel, sandy, brown

gravel and sand

clay hardpan, gray-brown

clay hardpan, gray

grave1, sandy

hardpan, clayey

NHW 241

clay, brown

clay, blue-gray

clay, black (few pebbles)

sand, clayey, brownish-

yellow

sand, coarse, brown

hardpan, gray

gravel, sandy, brown

sand, brown

grave1, sandy, brown

sand and gravel, white

quartz; 1ittle brown clayey

gravel

clay, white

sand, very fine, clayey,

white 
Table 2.--Lithologic logs of selected wells and test borings--Continued

\begin{tabular}{ll}
\hline Depth below & Description of \\
land surface & the sediment \\
(in feet) & \\
\hline
\end{tabular}

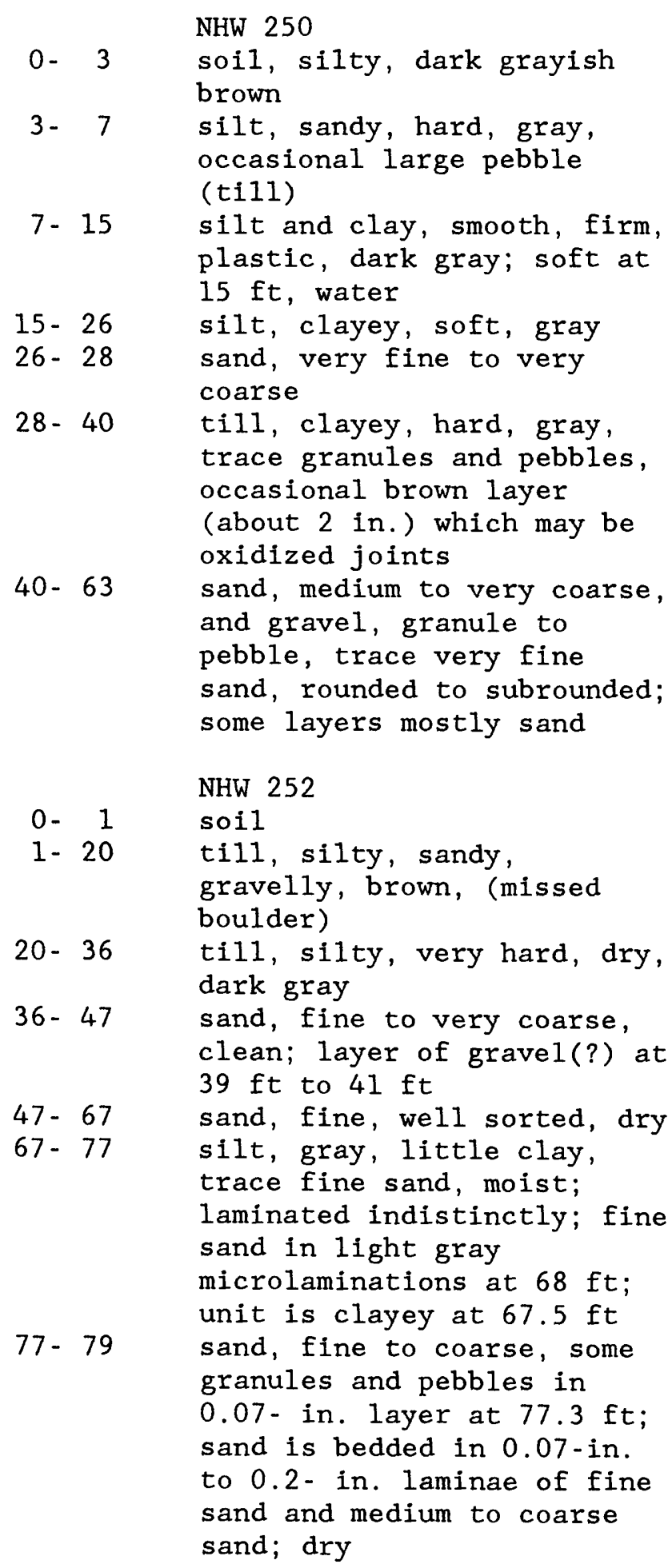

$\begin{array}{ll}\text { Depth below } & \text { Description of } \\ \text { land surface the sediment } \\ \text { (in feet) }\end{array}$

\begin{tabular}{|c|c|}
\hline $1-4$ & $\begin{array}{l}\text { NHW } 254 \\
\text { sand, medium to coarse, }\end{array}$ \\
\hline $4-5$ & $\begin{array}{l}\text { grave1, small pebbles ( } 1 \\
\text { in. to } 2 \text { in. diameter.) }\end{array}$ \\
\hline $5-20$ & $\begin{array}{l}\text { sand, medium to coarse, } \\
\text { brown, thin beds of very } \\
\text { coarse sand, granules, thin } \\
\text { silty clay }\end{array}$ \\
\hline $20-21$ & sand, silty, stiff (?) \\
\hline $21-40$ & $\begin{array}{l}\text { silt, gray and very fine } \\
\text { micaceous sand, laminated } \\
0.04-\text { in. to } 0.07-\text { in. layers, } \\
\text { sharp boundaries; some } \\
\text { laminae are oxidized; clayey } \\
\text { at } 39 \mathrm{ft} \text { to } 39.5 \mathrm{ft}\end{array}$ \\
\hline
\end{tabular}

$$
\begin{array}{rr}
0- & 1 \\
1- & 10 \\
10- & 13
\end{array}
$$

NHW 255

\section{soil}

till, clayey, silty, brown, some pebbles to cobbles sand, medium to very coarse, and gravel, granules to small cobbles (layer of cobbles at $13 \mathrm{ft}$ to $16 \mathrm{ft}$ ) till, silty, sandy, grayish brown, many cobbles 
Table 2.--Lithologic logs of selected wells and test borings--Continued

\begin{tabular}{ll}
\hline Depth below & Description of \\
land surface & the sediment \\
(in feet) & \\
\hline
\end{tabular}

\begin{tabular}{cl}
\hline Depth below & Description of \\
land surface & the sediment \\
(in feet) & \\
\hline
\end{tabular}

NHW $256,257,258$

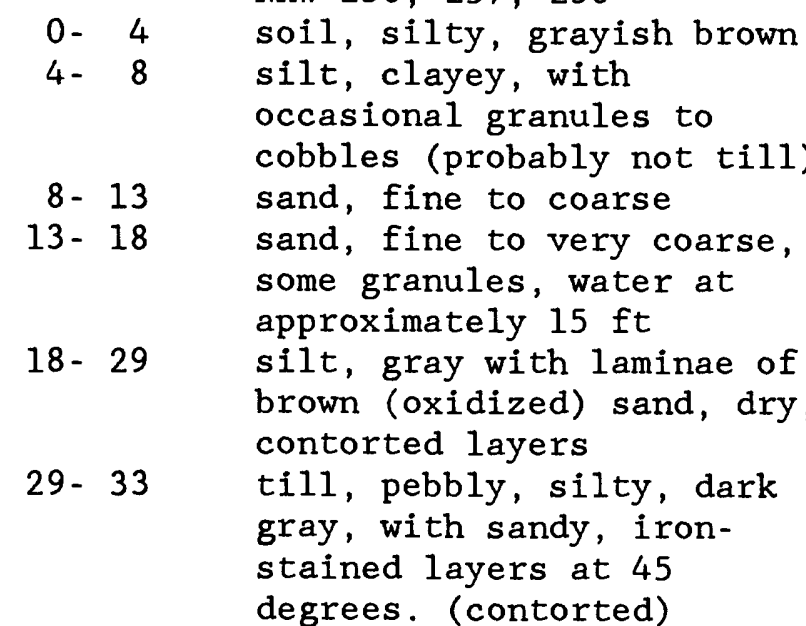

33- 37 sand, very fine to very

coarse and granule to pebble gravel

37- 41 till, pebbly

41- 43 sand, fine to very coarse, clean, saturated

43-57 till, silty, sandy, pebbly, brownish gray, bedded with sandier layers

57-84 clay, firm, dry, dark gray, with occasional pebble

84-87 sand, very fine to medium, some silt and very coarse sand, trace pebbles

NHW 259

0- 5 sand, fine to coarse, few pebbles

5- 9 till(?), sandy, red, with cobbles (may be ironcemented conglomerate)

9- 15 til1, stony, sandy

15- 37 sand (static water level 10 ft from $15 \mathrm{ft}$ to $17 \mathrm{ft}$ )

$37-47$

47- 64

$64-72$ sand, fine, white clay, red marbled with gray sand (heaving)
NHW 262

0 - 5 gravel, cobble to granule

5- 32 gravel, granule to cobble, and medium to very coarse sand

32- 63.5 sand, medium to very coarse, and granule to large pebble gravel

\section{$0-1$ \\ soil}

NHW 263

$1-20$

$20-43$

$43-58$

silt, some very fine sand

silt and very fine sand,

finely laminated, gray-brown and medium gray

sand, fine to very coarse, and granule to pebble gravel, trace very fine sand, iron-stained, dark reddish brown

58- 59 silt, gray, laminated, with interbedded 1 -in. layers of medium to very coarse sand

NHW 264

0 - 2 soil, sandy

2- 4 till, sandy and silty, gray

4- 15 till, silty, gray, occasional pebble to cobble

15- 21 sand, medium to very coarse, trace of fine sand

$21-31$

$31-33$

till, sandy, gray

sand, fine to very coarse,

trace of very fine sand

NHW 302

0 - 3 sand

3- 12 clay silt, brown

12- 15 clay, gray

15- 33 gravel hardpan

33- 55 gravel and sand

55- 72 clay hardpan

72 boulder

72- 82 cobbles, gravel

82-124 gravel, coarse 
Table 2.--Lithologic logs of selected wells and test borings--Continued

\begin{tabular}{ll}
\hline Depth below & Description of \\
land surface & the sediment \\
(in feet) & \\
\hline
\end{tabular}

NHW 303

$0-18$ grave 1

18- 60 sand, coarse

60- 67 sand, cobbly

67- 80 drill and lime

$80-90$

$98-131$

$0-7$
$7-16$
$16-63$
$63-70$
$70-90$
$90-126$

$0-1$

2- 7

$8-24$

$25-60$

$61-130$

$131-140$

$141-144$

$$
0-1
$$

$1-26$

$26-32$

$32-45$

$45-60$

$60-82$

$82-92$

$92-98$

$98-105$

$105-137$

$0-47$

$47-57$

$57-68$

$68-137$ gravel

gravel and sand, water

NHW 304

hardpan, cobbles

sand and gravel

hardpan, brown

sand and gravel

sand, finer

NHW 305

topsoil

loam sand, dark

sand and gravel, orange

sand and fine gravel

sand and fine quartz

sand, coarse

sand and gravel

NHW 307

topsoil, loam

sand

sand and gravel, some

cobbles

s and

sand and gravel, hardpan

seams

grave1, medium

hardpan, gravel

clay sand, brown

clay sand, orange

sand and gravel

NHW 308

sand

grave1 till

grave1

clay, brown

$\begin{array}{ll}\text { Depth below } & \text { Description of } \\ \text { land surface } & \text { the sediment } \\ \text { (in feet) } & \end{array}$

$0-1$

NHW 309

$1-5$

topsoil

7- 8

loam, sandy

9- 24

$24-25$

$26-32$

$32-33$

$34-38$

$39-43$

$43-93$

$0-12$

12- 19

19- 27

$27-30$

$30-40$

$40-42$

$42-44$

$44-56$

$56-58$

$58-60$

$60-103$

$0-10$

12

$10-50$

$50-53$

boulder

sand and small gravel

boulder

sand

boulder

sand and gravel

clay sand, brown

sand and gravel

NHW 313

brown gravelly

hardpan, gravelly

sand, clayey, brown

grave1, cobbly-boulder

grave1, brown

gravel, coarse

grave1, medium, brown

gravel, coarse

grave1, cobbly

boulder-gravel

gravel and sand

NHW 315

hardpan

boulder

gravel and sand, brown

sand, brown, little water

NHW 316

$0-1$

fill

$2-4$

$5-13$

$14-16$

$17-41$

$41-46$

topsoil and sand

sand and large gravel

cobbles

grave1, large, and cobbles

boulder

$0-48$

NHW 317

$48-74$ grave1

sand, medium coarse 
Table 2.--Lithologic logs of selected wells and test borings--Continued

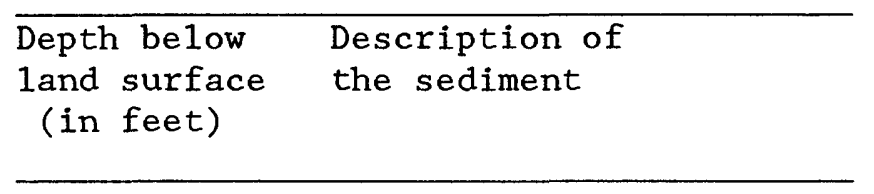

NHW 320

\begin{tabular}{|c|c|}
\hline & (n) \\
\hline $0-15$ & sand \\
\hline $15-17$ & sand, little gray clay \\
\hline $17-23$ & clay and sand \\
\hline $23-29$ & rock (boulder) \\
\hline $29-31$ & sand \\
\hline $31-35$ & sand and gray clay \\
\hline $35-40$ & clay and sand \\
\hline $40-57$ & clay and little sand \\
\hline $57-85$ & clay and sand \\
\hline $85-123$ & sand, coarse \\
\hline $123-143$ & $\begin{array}{l}\text { sand, fine and coarse and } \\
\text { clay }\end{array}$ \\
\hline $143-153$ & sand, fine and gray clay \\
\hline $153-161$ & $\begin{array}{l}\text { sand, coarse, sharp, fine } \\
\text { sand and gray clay }\end{array}$ \\
\hline $161-162$ & clay, soft \\
\hline $162-203$ & clay and sand \\
\hline $203-213$ & sand, fine, little clay \\
\hline $213-223$ & sand, sharp \\
\hline $223-228$ & sand, medium and very coarse \\
\hline $228-245$ & sand, coarse to very coarse \\
\hline & NHW 321 \\
\hline $0-30$ & sand \\
\hline $30-35$ & clay \\
\hline $35-110$ & sand \\
\hline 110 & c1ay \\
\hline & NHW 322 \\
\hline $0-20$ & sand, fine \\
\hline $20-31$ & clay and sand \\
\hline $31-78$ & grave1, fining to silt \\
\hline $78-149$ & sand \\
\hline $149-164$ & clay \\
\hline $164-180$ & gravel \\
\hline
\end{tabular}

\begin{tabular}{ll}
\hline Depth below & Description of \\
land surface & the sediment \\
(in feet) & \\
\hline
\end{tabular}

NHW 323

0 - 10 loam and boulders

10- 25 sand and grave 1

25- 32 clay and sand

32- 50 clay and grave1, little

water

50- 58 grave1, coarse, hard

58- 65 clay and grave1

65- 77 clay, b1ue

77- 88 grave1, coarse

88-102 clay, blue-black

102-119 gravel and brown sand

NHW 324

0-185 sand and coarse gravel

(whole way)

NHW 325

0- 1 topsoil

1- 4 sand, ye11ow brown

4- 8 sand, white

8- 26 clay, gray, gravel seams

$26-27$

$27-33$

$33-44$

$44-46$

$46-60$

$60-63$

$63-64$

$64-78$

$79-120$

$121-140$

$141-145$

$146-157$

$157-158$

$160-165$

$0-20$
$20-200$ boulder

grave1, hardpan, white sandy clay

sand, white

clay hardpan cobble

hardpan

hardpan gravel

clay

hardpan, gravel seams

hardpan, hard pack sand

hardpan, brown sand

clay, light brown, sand

hardpan, grave1

boulder

hardpan and grave1

cobbles and sand

clay, blue gray
NHW 328 
Table 2.--Iithologic logs of selected wells and test borings--Continued

\begin{tabular}{ll}
\hline Depth below & Description of \\
land surface & the sediment \\
(in feet) & \\
\hline
\end{tabular}

NHW 329

$\begin{aligned} 0-34 & \text { sand, clayey } \\ 34-43 & \text { hardpan } \\ 43-49 & \text { cobbles and boulders } \\ 49-54 & \text { hardpan, brown } \\ 54-65 & \text { grave1, coarse } \\ 65-87 & \text { sand and grave1 } \\ 87-90 & \text { sand, clayey } \\ 90-102 & \text { clay, gray } \\ 102-146 & \text { clay, brown } \\ 146-168 & \text { clay, sandy, brown } \\ 168-172 & \text { gravel and sand }\end{aligned}$

$\begin{aligned} 0-18 & \text { sand, fine } \\ 18-30 & \text { soil, clayey } \\ 30-50 & \text { clay } \\ 50-88 & \text { cobbles and brown gravel } \\ 88-130 & \text { clay, sandy, gray } \\ 130-140 & \text { clay } \\ 140-228 & \text { hardpan } \\ 228-232 & \text { gravel, sand } \\ 233 & \text { hardpan, clay } \\ & \text { NHW } 332 \\ 0-60 & \text { sand } \\ 60-280 & \text { hardpan, some solid layers } \\ & \text { of clay }\end{aligned}$

$\begin{array}{ll}\text { Depth below } & \text { Description of } \\ \text { land surface } & \text { the sediment } \\ \text { (in feet) } & \end{array}$

NHW 333

0- 15 silt-clay, 1ittle grave1, silty gravel

15- 18 sand, little gravel, silty sand

18- 25 gravel, little clay

$25-28$

$28-33$

$33-40$

$40-48$

$48-78$

$78-83$

$83-90$

90-103

$103-105$

105-108

$108-113$

$113-120$

$120-123$

$123-126$

$126-130$

$130-138$

$138-143$

$143-160$

$160-178$

$178-180$

$180-183$

183-192

192-198

$198-201$

201-231 gravel with clay

sandy

clay with gravel

clay, silt, some gravel

(drilling fast)

grave 1

silt and sand, fine

clay

clay, gray

clay, gray with some fines

clay, gray

clay, gray, with some gravel gravel

hard drilling

grave 1

gravel, silty, with clay

hard slow drilling

fines with some gravel, steady drilling

sand, coarse

sand, coarse to medium

gravel with clay

sand, fine

sand, very fine to medium clay

clay with hard fine sand gravel, hard slow drilling 
Table 2.--Lithologic logs of selected wells and test borings--Continued

\begin{tabular}{ll}
\hline Depth below & Description of \\
land surface & the sediment \\
(in feet) & \\
\hline
\end{tabular}

NHW 334

0 - 3 unknown

3- 6 sand, coarse

6- 12 sand with silt

12- 16 sand with gravel

16- 18 silt

$18-27$

$27-34$

$34-41$

$41-48$

$48-60$

$60-64$

$64-78$

$78-91$

$91-103$

$103-113$

$113-123$

$123-130$

$130-134$

$134-138$

$138-144$

$144-152$

$152-160$

$160-165$

$165-168$

$168-175$

$175-192$

$192-198$

$198-213$

213 sand with gravel

sand, coarse with gravel

sand, coarse

sand, medium to coarse

sand, very coarse

sand, coarse

sand, fine

clay, silty

sand, coarse, some clay

sand, medium

sand, medium to coarse

sand, coarse and gravel

grave 1

sand, fine to medium

sand, coarse

sand, medium to coarse

clay with gravel

clay

clay with some gravel and coarse sand

sand with clay

clay with gravel

sand, medium

sand, fine to medium, some

clay

clay, dark gray

$\begin{array}{ll}\text { Depth below } & \text { Description of } \\ \text { land surface } & \text { the sediment } \\ \text { (in feet) } & \end{array}$

NHW 335

$0-20$

20- 25

$25-32$

$32-40$

$40-52$

$52-55$

$55-58$

$58-60$

$60-64$

$64-70$

$70-73$

$73-103$

103-105

105-108

$108-115$

$115-133$

$133-136$

$136-148$

$148-153$

153-160

$160-170$

$0-41$

$41-57$

$57-64$

$0-49$

49- 63

$63-65$

$65-73$

73-105

$105-123$

gravel with clay

grave 1

gravel with fine sand

clay with gravel

clay, soft, easy drilling

clay, gray

clay with gravel

gravel with clay

gravel

sand, very coarse

sand, very coarse, some

gravel

sand, very coarse

sand, coarse

sand, fine

sand

sand, coarse

sand, fine with silt

clay with coarse sand

clay

sand, fine with clay

sand, coarse

NHW 336

cobbles, gravel, brown and green sand, gray sand

clay and sand

sand and grave1

NHW 337

cobbles and gravel

clay sand

clay

hardpan

clay

gravel

NHW 339

$0-7.5$ clay

$7.5-15.5$

15. 5-20

sand, coarse

sand, fine 
Table 2.--Lithologic logs of selected wells and test borings--Continued

\begin{tabular}{ll}
\hline Depth below & Description of \\
land surface & the sediment \\
(in feet) & \\
\hline
\end{tabular}

Depth below
land surface
(in feet)

NHW 340

$0-0.5$ topsoil

$7-60$

$60-69$

$69-70.2$

$70.2-75$

$75-90$

90-115

$115-136$

$136-162$

$162-168$

0- 11

$11-38$

$38-50$

$50-70$

$70-79$

$84-88$

$88-105$

105- 113

$113-148$

148-152

152

$$
\begin{array}{rr}
0- & 1 \\
2- & 5 \\
5- & 30 \\
31-64 \\
65-90 \\
91-97 \\
98-122 \\
122-134 \\
135-149 \\
150-153 \\
153-155 \\
155-163 \\
164-168
\end{array}
$$

cobbles

NHW 341

NHW 342

topsoil

grave 1 sandy loam, gray brown

Block Island sand and gravel

gravel and black sand layers

sand and gravel

sand, smal1 gravel

sand and gravel, medium

grave1, coarse

sand and gravel

hardpan, sandy, white

hardpan clay, gravel

gravel, sandy

sand, coarse

sand, fine, dark brown

sand, fine, dark

hardpan, brown

gravel, dirty, brown

gravel, coarse

sand, quartz, white

sand, quartz, gray

sand, gray white

sand, yellow, cobbles

sand and gravel

hardpan, sandy, brown

sand and gravel

grave 1, large, hard

clay sand, white

sand, quartz

sand, white (clay)

sand, quartz

clay, white

sand, quartz
NHW 343

0- 1 topsoil

2 - 4 loam sand

$5-10$

$11-15$

$16-18$

$18-24$

$25-37$

$38-42$

$42-46$

$47-49$

$49-55$

$56-66$

$67-70$

$70-75$

$76-82$

$83-86$

$87-93$

$93-100$

$100-129$

$129-139$

sand, 1ight (ye11ow)

clay sand, yellow

hardpan, gray

sand, white yellow

sand and fine gravel

hardpan sand, yellow

sand, light brown

hardpan, gray

hardpan gravel, gray

clay hardpan, gray

gravel, hard

hardpan gravel

gravel, sandy

gravel

sand hardpan, brown

clay, gray

clay sand, gray

sand and gravel

NHW 344

0 - 1 loam

2- 7 sand and cobbles

$7-21$

$22-23$

$24-37$

$38-44$

$44-45$

$46-70$

$71-85$

sand and gravel

boulder

grave1, coarse

sand

cobbles

gravel, coarse

sand and gravel

NHW 345

$0-7$ sand and gravel

7- 21 hardpan

21- 68 gravel 
Table 2.--Lithologic logs of selected wells and test borings--Continued

\begin{tabular}{ll}
\hline Depth below & Description of \\
land surface & the sediment \\
(in feet) & \\
\hline
\end{tabular}

\begin{tabular}{cl}
\hline Depth below & Description of \\
land surface & the sediment \\
(in feet) &
\end{tabular}

NHW 346

0- 10

10- 18

$18-38$

$38-53$

53- 70

70- 76

$76-85$

85- 96

96- 97

0-107

107-154

0- 21

$21-43$

43- 64

64- 80

80- 91

$91-96$

96-104

104-114

\section{0- 5}

5- 15

15- 20

$20-45$

$45-80$

$82-84$

84- 98

98-100

100-104

0- 10

10- 21

$21-84$

84- 87

$87-90$

90-102

$102-112$

kaolin

NHW 347

unknown

NHW 348

gravel

grave 1

grave 1

NHW 349

grave1

seams

boulder

cobbles

NHW 350 gravel, coarse

sand, coarse

sand, fine

sand, medium, brown

sand, dark brown

gravel, coarse

hardpan, gray

sand, gravelly

clay, red - kaolin seams

gravel, coarse

gravel - sand

grave1, sandy

clay sand

hardpan, clayey

topsoil - dirty sand

gravel, medium

sand and grave 1

clay sand, gray, with gravel

sand and small gravel

gravel and sand, medium

loam, sandy

gravel cobbles

gravel and hardpan

lens sand

hardpan, sandy

sand, fine, brown

grave1, sandy, water
$0-17$

$17-28$

$28-42$

$42-60$

NHW 353

$0-1$ loam

2 - 5 sand

6- 12 sand and grave1

13- 16 sand

17- 18 boulder

19- 30 hardpan, grave1

31- 33 boulder

34- 45 grave1, medium

46- 84 sand and gravel, hardpan

seams

NHW 354

gravel and sand

boulders and cobbles

gravel, coarse

sand and grave 1

NHW 355

0 - 7 hardpan

7- 30 gravel, coarse

$30-33$

33- 64

64

hardpan, gray

clay sand, gray

gravel and sand

NHW 356

$0-15$

$15-30$

$30-65$

clay, brown

clay, gray and sand

sand

NHW 358

$0-47$

47- 55

0- 11

$11-22$

$22-38$

$38-46$

46- 74

$0-15$

$15-35$

$35-55$

55- 64

\section{sand and clay}

sand

NHW 360

sand, silty

hardpan and sand

gravel, cobbly

grave 1

grave1, sandy

NHW 361

loam, sandy

gravel

sand

gravel, coarse 
Table 2.--Lithologic logs of selected wells and test borings--Continued

\begin{tabular}{ll}
\hline Depth below & Description of \\
land surface & the sediment \\
(in feet) & \\
\hline
\end{tabular}

\begin{tabular}{cl}
\hline Depth below & Description of \\
land surface & the sediment \\
(in feet) & \\
\hline
\end{tabular}

\section{NHW 362}

$\begin{aligned} & 0- 55 \\ & 55-62 \\ & 62-73 \\ & \\ & 0-13 \\ & 13-20 \\ & 20 \\ & 20-29 \\ & 29-63 \\ & 52\end{aligned}$

0.3

$3-6$

7. 10

10. 13

$13-20$

$21-23$

$24-27$

$28-35$

$35-36$

$37-45$

$45-47$

$48-51$

$51-54$

$54-65$

0- 15

$15-27$

$27-29$

$29-38$

$38-41$

$41-64$

0. 16

$16-22$

$22-66$

$0-41$

$41-53$

NHW 363

boulder

NHW 364

topsoil

boulder

boulder

gravel

NHW 365

gravel

cobbles

gravel

cobbles

gravel.

\section{NHW 366}

NHW 367

sand sand, fine, silty, brown

hardpan and sand, gray

sand and gravel

sand and gravel

gravel, coarse

gravel, coarse

sand and gravel

water and gravel

sand, yellow brown

gravel and sand

clay hardpan, gray

sand and gravel, brown

clay sand and stones

gravel and sand

gravel, large

gravel, large to medium with

hardpan seams

gravel, large

gravel and cobbles

clay sand

gravel, loamy

gravel, coarse

sand and gravel

sand, coarse, brown, water

NHW 370

$0-12$
$12-28$
$28-50$
$50-87$
$87-95$

$0-10$

$10-50$

$50-58$

$0-11$

$11-31$

$31-36$

$36-38$

$38-54$

54

$0-65$

$65-75$

75

$0-40$

$40-86$

$86-95$

95- 109

$109-116$

$116-139$

$139-148$

$148-155$

$155-161$

$0-1$

$1-6$

6- 20

$21-55$

$57-60$ gravel

gravel and cobbles

sand and gravel

sand, fine

sand, fine and gravel; fine

gravel and sand

NHW 372

sand and gravel, silty

hardpan, sandy, gray

sand, gravelly

NHW 373

gravel

sand, silty, black

sand, brown

cobbles

sand, clayey, black

sand and gravel, brown

NHW 374

sand and gravel

gravel and sand, brown

hardpan, gray

NHW 376

open hole - dug well

clay hardpan

gravel, clayey

gravel, clayey, soft

gravel and clay, some water

hardpan, clayey

hardpan, soft, clayey

sand, gray, water (set screen)

sand and gravel, water

NHW 377

topsoil

no record

sand, fine, yellow

sand and gravel

hardpan and gravel seam 
Table 2.--Lithologic logs of selected wells and test borings--Continued

Depth below
land surface the sediption of
(in feet)

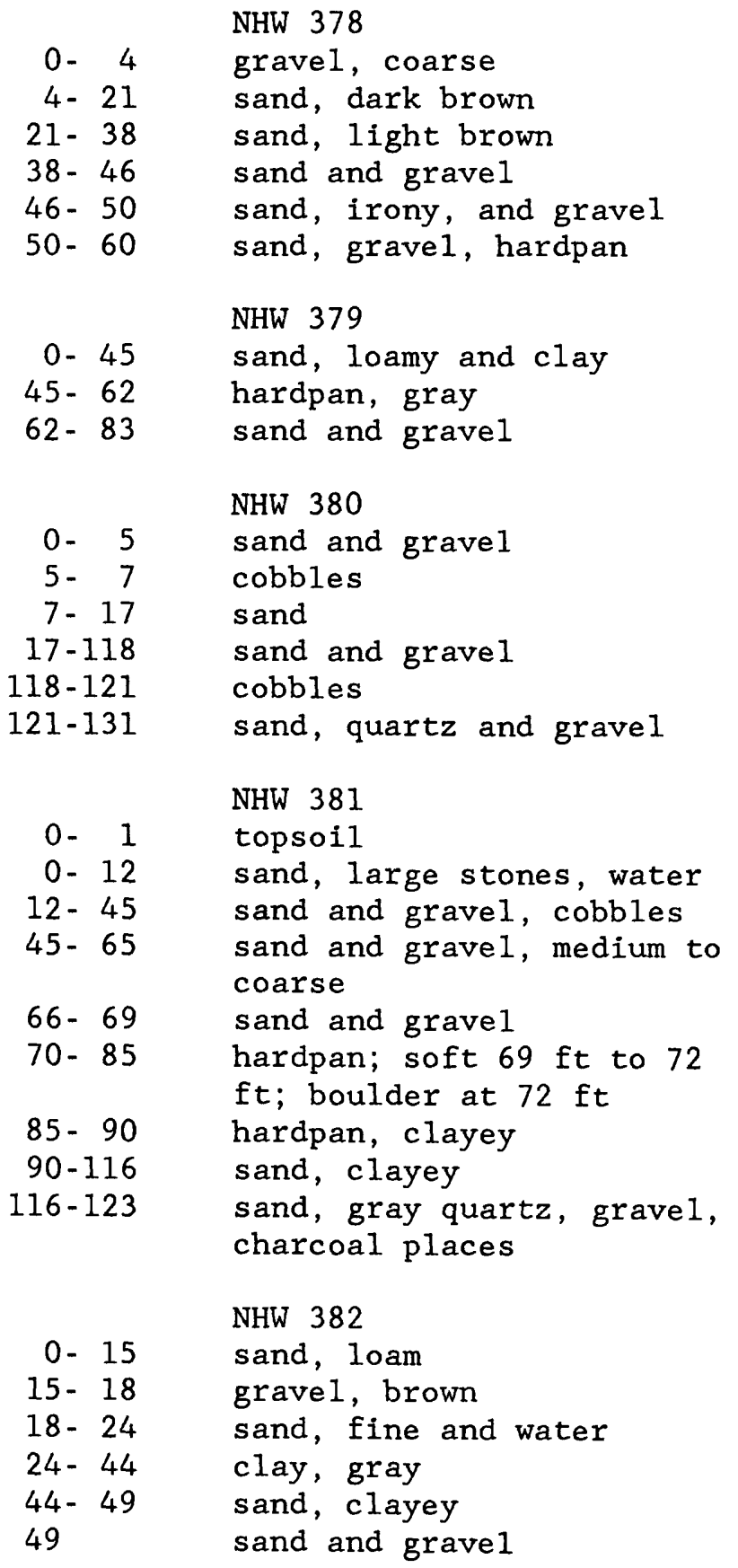

$\begin{array}{ll}\text { Depth below } & \text { Description of } \\ \text { land surface the sediment } \\ \text { (in feet) }\end{array}$

NHW 383

0- 28 gravel, coarse, cobbles (9

ft boulder, 18 ft boulder,

22 ft boulder)

28- 35 sand and gravel

35- 50 sand

$50-63$ grave 1

63- 80 hardpan, gray clay

80- 88 hardpan, sandy, gray

88- 92 sand, clayey, gray

$92-110$

$110-123$

clay, gray

grave1 and sand

NHW 384

0 - 3 fill, gravel, boulders

4- 16 gravel, brown

$16-32$

$32-34$

$35-53$

$54-59$

$59-64$

$65-67$

$67-69$

$70-79$

$79-86$

$0-25$

$25-54$

$54-60$

$60-65$

$65-70$

sand and gravel, gray

hardpan, gray clay

sand and gravel.

clay, gray and hardpan

sand and gravel

clay

boulder

clay hardpan

sand and gravel, brownyellow

NHW 385

grave1, boulder

sand, clayey

sand

clay sand

sand and gravel

NHW 386

0 - 5 sand, gray

5- 19 clay

19- 32 grave1

32- 38 clay sand

38- 61 sand and grave1

61- 69 clay sand

69-105 gravel and sand

$105-115$

$115-119$ sand

gravel and sand 
Table 2.--Lithologic logs of selected wells and test borings--Continued

\begin{tabular}{ll}
\hline Depth below & Description of \\
land surface & the sediment \\
(in feet) & \\
\hline
\end{tabular}

NHW 387

0 - 48 sand, fine, clayey, brown

$48-55$

$55-83$

$0-50$

50- 75

$75-100$

0. 6

6- 18

$18-34$

34- 54

54- 64

$0-19$

$19-40$

1. 8

8- 23

$23-42$

0 - 1

2- 4

4- 6

$7-30$

$31-48$

$48-63$

$63-77$

$78-90$

$90-106$

$106-112$

$112-118$

$118-128$

0- 19

19- 24

$24-43$

$43-60$ clay, blue-gray

gravel, coarse

NHW 389

clay

sand

clay

NHW 391

gravel

sand, cobble, boulder

sand, gravel

hardpan

sand, gravel

NHW 392

soil, clayey, brown

gravel, sandy

NHW 395

soil sand

gravel sand

sand

NHW 396

topsoil

sand, fine, brown

boulders

sand, coarse

sand, dark brown and water

muck sand, green

clay, pink

hardpan, gray, and sand

sand, gray

sand, gray white

sand, fine, white

sand, fine and coarse,

quartz, white; water

NHW 397

sand and gravel

sand, brown

sand

sand and gravel

$\begin{array}{ll}\text { Depth below } & \text { Description of } \\ \text { land surface } & \text { the sediment } \\ \text { (in feet) } & \end{array}$

NHW 400

$0-18$

$18-72$

$72-78$

$78-83$

grave 1

sand, dirty

hardpan

gravel, coarse

NHW 401

$0-8$

$8-18$

$18-26$

$26-42$

$42-49$

$49-63$

$63-105$

$105-112$

cobbles, gravel

grave 1, sand

hardpan, sandy, gray

sand, clayey

gravel, gray

hardpan, gray

sand and clay, gray

sand, clayey, gray, and

water

NHW 402

$0-7$

$7-18$

$18-24$

$24-56$

$56-70$

$70-82$

$82-101$

$101-110$

$110-112$

$112-140$

$140-145$

$0-42$

$42-45$

$45-52$

$52-53$

$54-64$

$64-66$

$66-74$

$74-76$

$76-85$

$85-98$

$98-103$

$103-146$

$146-152$

$152-157$ hardpan, gravelly

gravel

hardpan, gray

hardpan, sandy, gray

hardpan, gray

clay, sand

sand, fine, gray

hardpan

sand and gravel, green

clay, hard, blue

hardpan, gravel

NHW 403

silt, clayey

cobbles and clay

grave1, sand

sand, water

clay

gravel

clay

grave 1

clay

clay sand, fine

clay

hardpan

gravel and hardpan

gravel and sand 
Table 2.--Lithologic logs of selected wells and test borings--Continued

\begin{tabular}{ll}
\hline Depth below & Description of \\
land surface & the sediment \\
(in feet) & \\
\hline
\end{tabular}

$0-17$
$17-22$
$22-46$
$46-84$
$84-94$
$94-102$
$102-115$
$115-128$
$128-133.8$

\section{NHW 404}

sand, clayey, brown

hardpan, clayey, gray

hardpan

hardpan, sandy, clayey

gravel, coarse, brown

sand and gravel

sand, medium

sand, very fine brown

sand and gravel, water

NHW 405

0 - 0.5 topsoil

$0.5-4$

$5-15$

$15-80$

$80-124$

$124-126$

$126-143$

$0-42$

$42-143$

$143-150$

$150-158$

$158-160$

$160-178$

$0-5$

5- 10

$10-18$

$18-48$

$48-64$

$64-85$

$85-90$

$0-10$

$10-16$

$16-26$

$26-54$

$54-60$

$60-78$

$78-84$

sand and gravel

sand, gravel, cobbles

sand

clay, light gray

NHW 406

grave1, sandy

gravel, coarse; cobbles at

$90 \mathrm{ft}$

gravel, sandy

gravel, fine, sandy

layer hardpan

gravel and sand

NHW 407

loam and clay, gray

clay sand, brown

hardpan, gray

sand, medium coarse

sand, fine, gray and brown

clay, gray, and hardpan

sand, fine, brown

NHW 410

boulders, cobbles

grave1, cobbles

gravel, sandy

hardpan; clayey sand

hardpan, gravelly

hardpan, clayey

sand, brown

$\begin{array}{cl}\text { Depth below } & \text { Description of } \\ \text { land surface } & \text { the sediment } \\ \text { (in feet) } & \end{array}$

NHW 411

0- 15 gravel

15- 23 clay

23- 35 hardpan, clayey

35- 62 grave1, sandy, clayey

62- 71 gravel

$0-18$

NHW 415

$18-24$

sand

$24-30$

$30-42$

$42-52$

$52-68$

$0-18$

18- 23

sand, gravel (hardpan seams) 23- 45

sand, brown-black

clay sand, brown

sand, brown

hardpan

grave1

NHW 416

\section{sand, silty brown}

gravel

gravel and sand 
Table 2.--Lithologic logs of selected wells and test borings--Continued

\begin{tabular}{ll}
\hline Depth below & Description of \\
land surface & the sediment \\
(in feet) &
\end{tabular}

$0-1$
$1-\quad 4$
$4-9$
$9-10$
$11-14$
$14-30$
$30-34$
$35-37$
$37-40$
$40-56$
$57-58$
$58-59$
$60-67$
$68-75$
$75-80$
$83-86$
$86-91$
$91-105$
$105-110$
$110-141$
$142-150$
$151-153$
$154-164$
$164-190$
$191-197$
$197-207$
$208-216$
$216-218$
$219-227$
$228-247$

\section{NHW 417}

topsoil

sand

grave1, medium, orange

sand, light brown

clay, brown

sand and gravel

boulders and gravel

boulder

cobbles and grave1

sand and gravel

sand, clayey, gray

boulder

sand, clayey, gray

gravel and hardpan

gravel, medium large

sand, clayey, dark gray

sand

clay, gray

hardpan, clayey, gray

hardpan, clayey, red

sand and gravel

hardpan, clayey, red

hardpan, clayey, gray

sand, clayey, blue-gray

clay, gray

clay, blue-gray

sand and gravel

cobbles

sand and fine gravel

gravel, quartz and quartz

sand

\section{NHW 419}

0 - 9 sand, very coarse, and cobbles

9. 19

19. 28

$28-30$

$30-40$

$40-53$ grave 1, sandy

gravel and cobbles

cobbles

silt, brownish gray,

brownish gray sand

and brownish gray gravel

sand, fine to very coarse

\begin{tabular}{ll}
\hline Depth below & Description of \\
land surface & the sediment \\
(in feet) &
\end{tabular}

$0-5$

$5-31$

$31-48$

$48-70$

$70-93$

93-108

$108-213$

$213-239$

0 - 8

8- 18

$18-47$

62

$65-95$

95-106

$106-130$

$0-11$

$11-21$

$21-34$

$34-44$

36

$44-58$

$58-66$

$66-96$

$96-100$

100

$100-111$

$111-144$

$144-172$

$172-184$

$184-230$

$230-234$

$234-240$

$240-246$
NHW 422

clay

sand, medium (rock $16 \mathrm{ft}$ to

$17.5 \mathrm{ft}$ )

clay and sand

sand, coarse to medium

clay, blue-gray

clay, blue-gray, with fine

s and

clay, light gray, specks of

sand

sand

NHW 424

sand and loam

sand, clayey

hardpan, brown, clayey

boulder

silt, brown and water

hardpan, gray

gravel, clayey and clayey

sand

NHW 425

gravel and sand

s and

till

grave1

boulder

gravel and sand

sand, gray and gray clay

clay, gray

sand, clayey

water

sand and irony water

hardpan, gray and clay

sand and clay

sand

hardpan, clayey

sand, quartz, white

gravel, coarse, quartz

sand, fine, quartz and brown

water 
Table 2.--Lithologic logs of selected wells and test borings--Continued

Depth below
land surface the sediment
(in feet)

$\begin{array}{cl}\text { Depth below } & \text { Description of } \\ \text { land surface the sediment } \\ \text { (in feet) }\end{array}$

\section{NHW 426}

$0-11$
$11-16$
$16-23$
$23-41$
$41-68$
$68-76$
$76-78$
$78-96$
$96-104$
$104-128$
$128-131$
$131-137$

\section{grave 1}

sand and grave1

clay

gravel, clayey, brown

hardpan, gray

hardpan, gravelly

sand, gray, little water

hardpan, sandy

sand, clay and gravel

hardpan and clay

clay

sand and gravel, gray

NHW 428

\begin{tabular}{|c|c|}
\hline $\begin{array}{l}0-42 \\
40 \\
42-45 \\
45-62\end{array}$ & $\begin{array}{l}\text { gravel and sand } \\
\text { boulder } \\
\text { cobbles and sand } \\
\text { sand and gravel }\end{array}$ \\
\hline & NHW 431 \\
\hline $0-14$ & clay \\
\hline $14-15$ & sand \\
\hline $15-70$ & clay \\
\hline $70-77$ & $\begin{array}{l}\text { sand (confined with lots of } \\
\text { water) }\end{array}$ \\
\hline $77-78$ & clay \\
\hline
\end{tabular}

NHW 432

0 - 5 hardpan

5- 15 gravel, sandy

15- 35 hardpan, gray

35- 55 sand, clayey

63- 71 sand (set screen)

$71-82$

$82-112$

$112-123$

$\begin{aligned} 0-9 & \text { NHW } 433 \\ 9-18 & \text { grave1 } \\ 18-56 & \text { grave1, clayey } \\ 56-84 & \text { sand, fine, brown } \\ 84-107 & \text { clay, blue, cobbles } \\ 107-112 & \text { sand and gravel }\end{aligned}$

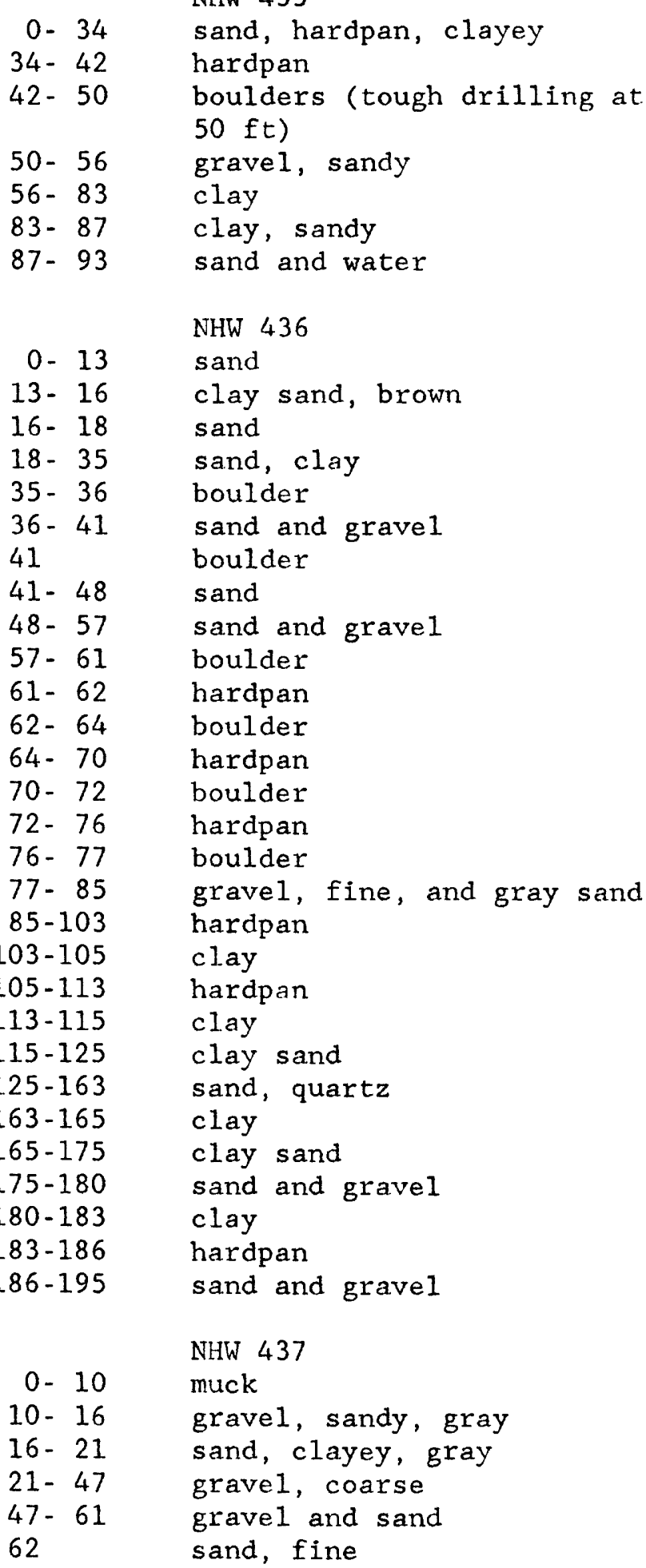

sand, hardpan, clayey

hardpan

boulders (tough drilling at $50 \mathrm{ft}$ )

clay

clay, sandy

NHW 436

sand

sand, clay

boulder

sand and gravel

boulder

s and

sand and gravel

boulder

hardpan

boulder

hardpan

boulder

gravel, fine, and gray sand

clay

hasdpan

clay

clay sand

sand, fine
NHW 435

clay, blue

clay

sand and gravel 
Table 2.--Lithologic logs of selected wells and test borings--Continued

Depth below Description of
land surface the sediment
(in feet)

NHW 438

$0-7$ sand

7 - 8 boulder

8- 14 sand and gravel

$14-17$

$17-31$

$31-36$

$36-43$

$43-48$

$48-54$

$54-61$

$61-62$

$62-67$

$67-68$

$68-88$

$88-89$

$89-108$
0. 6
$6-14$
$14-68$
$68-73$

$0-1$

$1-9$

9- 72

$72-88$

$88-105$

$106-120$

$121-135$

$136-150$

$150-160$

$160-178$

$178-185$

$185-195$

195-202 cobbles

sand and gravel

sand

clay sand

gravel and sand, fine

clay sand, brown

grave1, coarse

boulder

sand and gravel

boulder

sand and gravel

boulder

sand and gravel

NHW 440

topsoil

sand, fine

sand and gravel

NHW 443

topsoil

clay sand, gray

clay sands, gray and brown

boulders and gravel, clay

hardpan seams

clay sand and cobbles

hardpan gravel and sand

layers

clay, sandy

hardpan and gravel

hardpan and clay

clay, gray

sand, gray

hardpan gravel

sand and gravel hardpan sand, hard, gray

$\begin{array}{ll}\text { Depth below } & \text { Description of } \\ \text { land surface } & \text { the sedinent } \\ \text { (in feet) } & \end{array}$

$\begin{array}{rr}0- & 4 \\ 4- & 5 \\ 5- & 6 \\ 6- & 7 \\ 7- & 9 \\ 9- & 10 \\ 10- & 13 \\ 13- & 14 \\ 14- & 15 \\ 15- & 16 \\ 16- & 17 \\ 18- & 19 \\ 19- & 22 \\ 22- & 24 \\ 24- & 28 \\ 28-58 \\ 58-60 \\ 60-63 \\ 63-65 \\ 65-67 \\ 67-69 \\ 69-72 \\ 72\end{array}$

$0-48$

$48-52$

$52-65$

$65-72$

$72-88$

$88-126$

$126-136$

$136-140$
NHW 449

s and

boulder

sand and gravel

boulder

cobbles and gravel

boulder

sand and grave 1

boulder

sand and grave1

boulder

grave1, sandy

sand and gravel, gray

clay, gray

hardpan

clay sand

sand and gravel

boulder

sand and grave 1

boulder

sand and gravel

boulder

sand and gravel

boulder

NHW 450

grave1, coarse

sand, clayey

clay

hardpan

gravel, coarse, brown

sand, brown

sand, fine, clayey

sand, quartz, gray 
Table 2.--Lithologic logs of selected wells and test borings--Continued

Depth below Description of
land surface the sediment
(in feet)

\begin{tabular}{ll}
\hline Depth below & Description of \\
land surface & the sediment \\
(in feet) & \\
\hline
\end{tabular}

\section{NHW 451}

$0-31$
$31-38$
$38-46$
$46-55$
$55-62$
$62-88$
$88-100$
$100-114$
$114-132$
$132-149$
$149-161$
$161-173$
$173-210$
$210-213$
$213-220$
$220-220+$

$0-68$

$68-118$

$118-135$

$135-165$

$165-168$

$168-202$

$202-210$

$210-232$

$232-235$

$$
\begin{array}{r}
0-18 \\
18-24 \\
24-39 \\
39-65 \\
65-69 \\
69-106 \\
106
\end{array}
$$

gravel

NHW 452

sand

grave 1

hardpan

hardpan

NHW 453 silt, loam, little clay

hardpan, gray

gravel, coarse, brown

gravel, high iron

drill \& drive

grave1, brown

sand, quartz

sand, fine, brown

sand, fine, black

sand, fine, gray

sand, gray, hardpan

clay, gray

hardpan, gray

sand and gravel, fine

clay, silty, brown black

sand, brown

clay sand, fine

clay and hardpan

hardpan, cobbles

sand and water

grave 1, sandy

clay, gray

hardpan, gray

clay, sandy

hardpan, gray

sand and gravel, brown

sand and gravel - water
$0-15$
$15-37$
$37-42$
$42-65$
$65-93$
$95-110$
$110-122$
$122-129$
$129-151$
$151-154$
$154-162$

$0-16$

$16-21$

$21-42$

$42-49$

$49-65$

$65-70$

$70-73$

$0-1$

$1-2$

$2-15$

15- 18

$18-33$

$33-48$

$48-53$

$53-63$

$0-46$

$46-62$

$62-74$

$74-89$

$0-11$

$11-26$

$26-45$

$45-53$

$53-60$
NHW 455

gravel

sand and grave1

hardpan

gravel, coarse

sand, clayey

clay, brown

sand, fine, brown

clay sand, gray

clay sand, brown

hardpan, brown

gravel and water

NHW 461

gravel and loam

cobbles and gravel

grave1, coarse

hardpan, sandy, gray, clay

clay, hardpan

gravel, cobbles

cobbles, gray sand

NHW 463

dirt, black

dirt, brown

clay and sand

sand, quartz, heavy - spots of clay

sand, white (rock or cobbles at $28 \mathrm{ft}$ to $30 \mathrm{ft}$ )

sand, fine

sand and gravel, "heavy

mix", lots of quartz grains

sand and gravel

NHW 465

hardpan

sand

hardpan

sand gravel

NHW 466

grave 1 and sand

hardpan

hardpan and sand

sand, fine, brown-white

sand, medium coarse and fine 
Table 2.--Lithologic logs of selected wells and test borings--Continued

\begin{tabular}{ll}
\hline Depth below & Description of \\
land surface & the sediment \\
(in feet) & \\
\hline
\end{tabular}

\begin{tabular}{cl}
\hline Depth below & Description of \\
land surface & the sediment \\
(in feet) & \\
\hline
\end{tabular}

NHW 503

0 - 3 till (possibly fill)

3- 9 sand, light brown

9- 18 sand, fine and grave1

boulder

sand and gravel, fine

sand, gray, fine

sand, brown and gray, fine

sand and fine gravel

sand and gravel

NHW 502

$0-1$ topsoil

1- 8 loam grave1

$8-12$

$12-30$

$30-50$

$50-75$

$75-96$

$96-101$

$102-104$

$105-112$

$113-116$

$116-124$ clay, sandy, gray

sand and gravel

gravel, medium

sand and gravel

sand, medium

hardpan and clay

sand and gravel

clay

hardpan, sandy

grave1, medium
$18-30$

$31-53$

$53-54$

$54-61$

$62-63$

$63-76$

$76-79$

$79-87$

$87-91$

$91-94$

0. 11

$11-16$

$17-23$

24

$25-45$

$46-64$

$65-67$

$68-76$

$76-82$

gravel and sandy gray clay

sand and grave 1

cobbles

hardpan, gray and gravel

cobbles

gravel seams - gray clay and

brown hardpan

clay sand, brown

gravel and sand (lost mud)

sand and gravel, fine, gray

sand and gravel

NHW 504

hardpan, sandy, gray;

cobbles at $3 \mathrm{ft}$

clay sand, gray

sand and grave1

cobbles

sand, gravel, some cobble

sand, fine and medium

clay sand, gray

sand

sand and gravel

NHW 505

$0-20$

20- 26

26

$26-28$

$28-163$

$163-164$

$164-169$

$169-189$

$189-206$

$206-225$

$225-234$ gravel and lens clay

hardpan

boulder - cobbles

clay, hardpan, boulders

gravel, sandy

kaolin

hardpan clay, gray

clay, gray

gravel clay

clay, blue gray

sand, quartz 
Table 2.--Lithologic logs of selected wells and test borings--Continued

Depth below
land surface the sediment
(in feet)

NHW 506

$0-4$ sand

4- 6

$6-8$

$8-11$

$11-13$

14- 15

15- 18

18

$19-23$

24

25- 30

30

$31-38$

$38-40$

$40-44$

44- 54

$54-93$

$94-110$

111-145

$145-160$

$160-168$

$169-177$

$178-180$

195

199-207

0- 18

$18-28$

$28-42$

$42-60$

$60-73$

$73-77$

$77-82$

$82-90$

$90-96$

$96-144$ sand and gravel

boulder

gravel and hardpan

sand and gravel

cobbles

sand and gravel

cobbles

sand, coarse, hardpan

cobbles

hardpan, gravel

boulder

hardpan, boulder

boulder and cobbles

grave1, coarse

grave1 and cobbles, coarse

gravel, coarse, large

grave1, large

grave1, hardpan seams

grave1, gray

hardpan and clay

grave1

clay sand, gray

cobbles and clay

grave 1

NHW 508

grave 1

grave1 and sand

grave1, sandy

sand

sand, gray

clay

sand, clayey

clay sand, brown

grave1 and sand, brown

sand
Depth below
land surface
Description of

(in feet)

$$
\begin{array}{rr}
0- & 6 \\
6-11 \\
11-15 \\
15-20 \\
21-45 \\
46-54 \\
55-57 \\
57-64 \\
65-68 \\
69-71 \\
72-91 \\
98-103 \\
104-107 \\
108-115 \\
115-151 \\
151-155 \\
156-175 \\
176-187 \\
187-196 \\
197-204 \\
205-215
\end{array}
$$

$0-5$

$5-9$

9- 15

$15-26$

26- 41

$41-43$

$43-45$

45

0- 18

$18-30$

$30-32$

$32-35$

$35-42$

$42-129$

129-227

$227-228$

$228-233$

$233-245$
NHW 509

topsoil

sand, light brown

cobbles

clay sand, yellow

sand and gravel

hardpan gravel

boulder

hardpan gravel

clay hardpan

sand and gravel

clay sand

sand and small gravel

clay sand, green clay

hardpan, sandy, brown

sand - hardpan gravel seams

clay sand, fine, brown

clay sand

clay

clay hardpan

clay, sandy

sand and gravel

NHW 511

sand and grave 1

grave1, black, and oil

sand, black muck

clay and sand, blue

clay, hard, black

sand, clayey, black

clay, gravelly, w/water

gravel and quartz, gray

NHW 512

hardpan

clay sand

clay

gravel

cobbles and sand

grave 1

clay sand and clay

boulder

clay

sand and gravel 
Table 2.--Lithologic logs of selected wells and test borings--Continued

Depth below Description of
land surface the sediment
(in feet)

NHW 514

$\begin{aligned} 0-2 & \text { loam } \\ 2-12 & \text { grave1, sandy } \\ 12-34 & \text { sand, clayey } \\ 34-44 & \text { grave1ly } \\ 44-60 & \text { hardpan, sandy } \\ 60-136 & \text { hardpan, clayey } \\ 136-142 & \text { hardpan, sandy } \\ 142-150 & \text { sand, fine and water }\end{aligned}$

\section{NHW 515}

$0-5$

$5-8$

$8-13$

$13-16$

$16-20$

$21-25$

$25-41$

$41-51$

$51-71$

$71-73$

$73-77$

$77-141$

141-149

$0-14$

14- 25

25

$25-32$

$32-45$

$45-54$

$0-7$

$8-25$

$25-27$

$28-36$

$36-55$

$55-60$

$61-64$

65- 76

$77-127$ hardpan, brown

cobbles, large

sand and gravel

clay, gray

hardpan, gravelly

boulder

sand and gravel

gravel, hardpan seams

clay, gray

hardpan, gray

sand, gray

clay and hardpan, gray

gravel and sand

\section{NHW 516}

cobbles and hardpan

hardpan, gray

boulders, hardpan

hardpan, brown

clay sand, gray

clay, grave1ly

\section{NHW 517}

fill, sand, and loam

sand and dirty gravel

clay hardpan, gray

hardpan, stones and gravel

sand and gravel

gravel

grave1, large

sand and small gravel

sand and gravel

\author{
Depth below \\ Description of \\ land surface \\ the sediment \\ (in feet)
}

$0-11$
$11-55$
$55-65$
$65-74$
$74-82$
$82-98$
$98-103$

0- 1

1- 9

$11-13$

$13-33$

$35-37$

$37-40$

$40-79$

$79-81$

$82-124$

$0-4$

4- 8

$8-22$

$22-40$

$40-49$

49- 62

$62-73$

73- 79

$0-2$

3- 20

21- 26

27- 35

$36-44$

$45-61$

$62-84$
NHW 520

sand, loamy, and grave 1

sand, clayey

cobbles, gravel, brown

gravel

sand

sand, fine

sand

NHW 521

topsoil

sand and gravel, gray

boulder

clay hardpan, gray

sand and gravel

clay sand, light brown

grave1 and hardpan seams

clay, gray and brown

sand and gravel

NHW 522

cobbles, gravel

gravel

hardpan, gravelly

grave1, sandy

sand, fine, gray

sand hardpan, gray

hardpan

sand and gravel

NHW 523

topsoil, dark brown

clay, sandy, gray

grave1, gray

clay and cobbles

clay, gray

gravel, medium and small

sand and gravel 
Table 2.--Lithologic logs of selected wells and test borings--Continued

\begin{tabular}{l} 
Depth below $\begin{array}{l}\text { Description of } \\
\text { land surface the sediment } \\
\text { (in feet) }\end{array}$ \\
\hline
\end{tabular}

Depth below Description of
land surface the sediment
(in feet)

\begin{tabular}{|c|c|c|c|}
\hline $0-3$ & $\begin{array}{l}\text { NHW } 524 \\
\text { soil boulder }\end{array}$ & & NHW 529 \\
\hline $3-14$ & hardpan, gray & $1-4$ & $\begin{array}{l}\text { sopsoli } \\
\text { sand, light brown }\end{array}$ \\
\hline $14-26$ & grave1, sandy & $4-9$ & gravel and sand, brown \\
\hline $26-74$ & grave1, coarse & $9-30$ & gravel and hardpan \\
\hline $74-90$ & sand and gravel, gray & $30-33$ & clay \\
\hline $90-101$ & sand, fine, gray, white & $34-75$ & sand and gravel hardpan \\
\hline $101-129$ & sand, fine, dirty, gray & $76-79$ & sand \\
\hline $129-150$ & clay, gray & $80-87$ & sand and grave 1 \\
\hline $150-158$ & clay, sandy, gray & $88-94$ & sand, dark \\
\hline $158-188$ & clay, gray & $95-101$ & clay \\
\hline $188-193$ & $\begin{array}{l}\text { sand and gravel, quartz - } \\
\text { water }\end{array}$ & $\begin{array}{l}101-106 \\
107-130 \\
130-133\end{array}$ & $\begin{array}{l}\text { gravel sand and clay seams } \\
\text { sand and gravel } \\
\text { clay sand }\end{array}$ \\
\hline $0-1$ & $\begin{array}{l}\text { NHW } 526 \\
\text { topsoil }\end{array}$ & $134-144$ & sand grave 1 \\
\hline $1-9$ & sand and gravel, brown & & NHW 530 \\
\hline $9-54$ & sand and gravel, orange & $0-4$ & grave1, coarse \\
\hline $55-104$ & cobbles and grave1 & 4- 16 & sand \\
\hline $105-114$ & clay sand & $16-44$ & hardpan, light \\
\hline $114-130$ & s and & $44-99$ & grave1, medium \\
\hline $130-148$ & sand, brown and gray & $99-105$ & grave1, coarse \\
\hline $148-215$ & clay & $\begin{array}{l}105-128 \\
128-132\end{array}$ & clay sand, hard \\
\hline & NHW 527 & $132-134$ & gravel, brown \\
\hline $0-6$ & sand & 134 & grave1, coarse \\
\hline $6-18$ & sand, clayey & & \\
\hline $18-37$ & clay, blue & & NHW 532 \\
\hline $37-45$ & hardpan, brown & $0-9$ & loam and cobbles, clayey \\
\hline $45-60$ & gravel, brown & $9-24$ & clay hardpan \\
\hline $60-66$ & sand, brown & $24-43$ & gravel, brown \\
\hline $66-90$ & hardpan, gray & $58-62$ & hardpan, boulder \\
\hline $90-175$ & clay, gray (hard driving) & $62-68$ & hardpan, brown \\
\hline 175 & sand, quartz & 68 & grave1, brown \\
\hline & $\begin{array}{l}\text { NHW } 528 \\
\text { sand }\end{array}$ & & NHW 533 \\
\hline $\begin{array}{r}0-22 \\
22-41\end{array}$ & $\begin{array}{l}\text { sand } \\
\text { clay and hardnan }\end{array}$ & $0-46$ & silt and sand \\
\hline $41-121$ & $\begin{array}{l}\text { clay and hardpan } \\
\text { graye }\end{array}$ & $46-56$ & clay, gray \\
\hline $121-143$ & gravel & $56-137$ & gravel and brown sand \\
\hline $121-143$ & clay and sand & $137-146$ & sand, finer, brown \\
\hline $143-153$ & no record & $146-155$ & sand, water \\
\hline $153-173$ & clay & & \\
\hline $173-190$ & gravel & & \\
\hline
\end{tabular}


Table 2.--Lithologic logs of selected wells and test borings--Continued

\begin{tabular}{cl}
\hline $\begin{array}{c}\text { Depth below } \\
\text { land surface } \\
\text { (in feet) }\end{array}$ & $\begin{array}{l}\text { Description of } \\
\text { the sediment }\end{array}$ \\
\hline
\end{tabular}

\begin{tabular}{cl}
\hline Depth below & Description of \\
land surface & the sediment \\
(in feet) & \\
\hline
\end{tabular}

NHW 534

$\begin{array}{rr}0- & 4 \\ 4- & 18 \\ 18- & 27 \\ 27- & 35 \\ 35- & 76 \\ 76-85 & \\ 85 & \end{array}$

$0-26$

$26-48$

$48-63$

$63-80$

$80-83$

$83-90$

$90-112$

$112-134$

$134-150$

150

$150-163$

$0-20$

20- 45

$45-65$

$65-80$

$80-108$

$108-120$

$0-8$

8- 26

$26-48$

$48-53$

$0-120$

NHW 540

NHW 535

loam, silty

clay, silty, gray

hardpan, clayey, gray

gravel, clayey, brown

gravel, sandy, brown

sand, brown

sand, fine, brown

sand and gravel, loamy

sand and gravel

sand, brown

hardpan and sand, gray

sand, clayey, gray

sand, clayey, gray brown

sand, clayey, little water

clay sand, brown

sand, brown and gray

little water

sand and gravel, fine

NHW 538

boulders

gravel

gravel, sandy

grave1, coarse

stones and gravel

gravel, coarse

NHW 539

gravel

sand and gravel

sand

coarse ${ }^{1}$

clay, sand, boulder

NHW 541

$0-6$

6- 14

$14-38$

$38-66$

$66-83$ gravelly

clay and boulders

clay and hardpan

sand, gravelly

sand - water

$$
\begin{array}{rr}
0- & 8 \\
8- & 24 \\
24-62 \\
62-74 \\
74-97 \\
97-106 \\
106-144 \\
144-178 \\
178-181 \\
181-196 \\
196-213 \\
213-216
\end{array}
$$

0- 4

4- 8

$8-54$

$54-60$

$60-63$

$63-65$

$65-79$

$79-81$

$81-82$

$82-83$

$83-86$

$86-87$

$87-89$

$89-90$

$90-94$

$94-128$

$128-155$

$155-198$

$198-224$

$0-32$

$32-45$

$45-70$

70
NHW 542

sand, loamy

sand, gray, little clay

sand, brown

gravel and sand

clay, gray

sand, clayey, gray

clay, gray

clay, little sand

gravel, gray

hardpan, gravelly

clay and hardpan, sandy

sand and gravel, clayey

NHW 543

sand and gravel

clay

sand and gravel

clay

sand and grave1

boulder

hardpan

boulder

sand

boulder

sand and gravel

boulder

sand, brown

boulder

sand and gravel, brown

sand and gravel

sand and gravel, gray

hardpan

grave1

NHW 544

clay and gravel, sandy

clay, blue

sand, clayey

gravel, sandy

\section{material}

$1_{\text {Driller's }}$ log did not identify 
Table 2.--Lithologic logs of selected wells and test borings--Continued

\begin{tabular}{ll}
\hline Depth below & Description of \\
land surface & the sediment \\
(in feet) &
\end{tabular}

NHW 545

0 - 6 hardpan

6 boulder

6- 18 clay

$18-34$

$34-38$

$38-42$

$42-89$

89- 117

$117-121$

$121-126$

$126-141$

$141-146$

$0-13$

$13-28$

$28-32$

$32-36$

$36-38$

$38-70$

$70-78$

$78-96$

$96-101$

101-109

109-111

$111-135$

$135-138$

$138-142$

$142-143$

\section{clay and stones}

sand, brown

clay hardpan, brown

clay, gray

sand, fine, brown

sand, fine, gray

hardpan

sand, gray

sand, coarse, gray, water

NHW 546

gravel, fine

cobbles and gravel

boulders, small

gravel, coarse

boulders

gravel and sand, fine

hardpan, gray

gravel lens sand

cobbles, gravel

gravel, fine

cobbles, gravel

grave1, fine

gravel seams, clay sand

grave1, coarse

clay sand, gray

\section{NHW 547}

clay and hardpan

dry seam, 2 boulders

water and gravel, brown sand

Depth below Description of
land surface the sediment

(in feet)

$\begin{array}{rr}0- & 9 \\ 10 & -14 \\ 9-14 \\ 14-41 \\ 41-68 \\ 68-86 \\ 86-93 \\ 93-105 \\ 105-118 \\ 118-135 \\ 135-150 \\ 150-155\end{array}$

$\begin{array}{rr}0- & 4 \\ 4- & 9 \\ 10- & 16 \\ 16- & 30 \\ 31- & 45 \\ 46- & 65 \\ 66-72 \\ 73-83 \\ 84-96 \\ 96-100 \\ 101-127 \\ 127-130\end{array}$

0. 8

$8-20$

$20-34$

$34-66$

$66-72$

$72-84$

84- 96

$0-18$

18- 24

$24-46$

$46-49$

$49-60$

$60-65$

$65-80$

$80-104$
NHW 548

sand and gravel

boulder

hardpan

sand and gravel

sand, fine

sand, fine gray

clay, brown

gravel, coarse

gravel and sand

sand, fine, brown

silt sand, fine, gray

sand and gravel

NHW 549

loam, sand

sand, brown

hardpan, gray

grave1, dirty

grave1, medium with hardpan

gravel and sand with hardpan

clay, brown

grave1, hard mud

sand and gravel, hard

clay sand, gray

sand and grave 1

gravel, medium

NHW 550

sand and grave1

hardpan, gray

clay and sand

hardpan, clayey

hardpan, gravelly

sand and clay, gravelly

sand

NHW 551

gravel, cobbles

sand

clay, gray

cobbles

hardpan, gray

gravel, gray - cobbles

sand and gravel, gray

sand and gravel, brown 
Table 2.--Lithologic logs of selected wells and test borings--Continued

\begin{tabular}{ll}
\hline Depth below & Description of \\
land surface & the sediment \\
(in feet) &
\end{tabular}

NHW 552

$0-12$

$12-45$

$45-59$

$59-62$

$62-75$

$75-105$

$105-115$

$115-124$

$124-127$

$127-135$

$135-158$

167

170

$172-191$

191-194

\section{$0-6$}

$6-40$

$40-118$

$118-122$

$122-131$ grave 1

sand, brown

sand, gray

sand and gravel, brown

hardpan, gray

clay, fine

grave1

hardpan, gray

gravel, sandy

hardpan, gray

clay, blue

sand and water

set screen - no water

sand, clayey

gravel

NHW 553

gravel

hardpan

sand, fine, brown

sand, fine, silty, brown

sand, fine, quartz, clayey

\begin{tabular}{cl}
\hline Depth below & Description of \\
land surface & the sediment \\
(in feet) &
\end{tabular}

NHW 554

0 - 1 loam

1- 4 sand, brown

4- 5 boulder

5- 7 sand

8- 10 boulder

$11-15$ sand

15- 16 boulder

$17-25$

$26-28$

$29-55$

$56-63$

$64-103$

104-106

$107-126$

$126-128$

$129-142$

$142-144$

$144-146$

$147-168$

$169-171$

$172-182$

$183-193$

$193-199$

$199-200$

$0-18$

$18-43$

$43-47$

$47-74$

$74-88$

$88-120$

$120-145$ cobbles and sand

clay

cobbles and gravel

sand and gray clay

sand and medium gravel

clay hardpan

sand and gravel

clay sand, brown

sand, fine gravel

clay sand

sand, hardpan, gray

sand and gravel

hardpan, gray

clay and gravel, brown, sand seams

clay, gray

gravel, coarse

hardpan

NHW 555

hardpan, sandy

sand, clayey gray

hardpan, clayey

clay, blue

gravel, coarse, brown

gravel, coarse, sandy

sand and gravel 
Table 2.--Lithologic logs of selected wells and test borings--Continued

\begin{tabular}{ll}
\hline Depth below & Description of \\
land surface & the sediment \\
(in feet) &
\end{tabular}

NHW 556

$0-1$ topsoil

1 - 4 sand, yellow

4- 6 sand and cobbles

6- 25 sand

$25-38$

$38-43$

$43-50$

$50-58$

$58-91$

$91-110$

$110-132$

$132-134$

$134-146$

$146-208$

$208-222$

$0-36$

$36-58$

$58-65$

$65-90$

$90-140$

$140-164$

$164-185$

$185-188$

$188-190$

$190-206$

$206-215$

$215-248$

$248-259.5$

$$
\begin{array}{rr}
0- & 4 \\
4- & 22 \\
22-95 \\
95-116 \\
116-130 \\
130-181 \\
181-210 \\
201
\end{array}
$$

sand and grave 1

gravel and cobbles

sand and grave 1

clay

sand and gravel

grave1, coarse

sand and gravel

sand and gravel, gray

clay sand, gray

clay, gray

sand and grave1

NHW 557

sand

clay, gray

hardpan

sand, brown

sand, fine, brown, water

sand, fine, brown

clay

hardpan, brown

grave1, medium

hardpan, gravelly

hardpan - coarse gravel

hardpan and dirty gravel

sand and gravel

NHW 558

sand

hardpan

grave 1 and sand

sand, fine, gray

sand, clayey, gray

clay, blue gray

hardpan, gravelly

grave1, water

$\begin{array}{cl}\text { Depth below } & \text { Description of } \\ \text { land surface } & \text { the sediment } \\ \text { (in feet) } & \end{array}$

NHW 559

0- 7

$7-40$

$40-56$

$56-63$

$63-68$

$68-78$

$78-102$

$102-112$

$112-138$

$138-150$

$150-184$

$184-190$

190-201

201-214

214-217
$0-6$
$6-8$
$8-26$

$26-34$

$34-45$

$45-60$

$60-100$

$100-177$

$177-220$

$220-231$

$231-238$

$238-243$

$243-247.8$

grave 1, coarse

gravel and hardpan, sandy

sand, clayey, brown

sand, clayey, gray

grave1, sandy

sand, gray

grave1, sandy, brown

clay, gray

hardpan, gray

hardpan, sandy

clay

clay sand

sand, fine gray

sand and gravel, fine, silty

sand and grave 1

NHW 560

grave 1

boulders

grave1, cobbles

gravel, sandy

sand, clayey, gray

hardpan

clay, sandy, gray

clay, gray

clay hardpan

sand and gravel

hardpan, sandy

hardpan, sandy, gray

(driving very hard)

gravel, sandy

$0-89$

89- 109

$109-198$

198-202

$202-215$

$215-238$

$238-240$
NHW 561

grave 1

clay, sandy

clay

clay and hardpan

hardpan, coarse

gravel and hardpan

sand and gravel 
Table 2.--Lithologic logs of selected wells and test borings--Continued

Depth below
land surface
(in feet)

\begin{tabular}{ll}
\hline Depth below & Description of \\
land surface & the sediment \\
(in feet) & \\
\hline
\end{tabular}

NHW 562

$\begin{array}{rr}0- & 1 \\ 1- & 3 \\ 3- & 5 \\ 5- & 7 \\ 7- & 12 \\ 12- & 15 \\ 16- & 26 \\ 26-75 \\ 75-85 \\ 85-147 \\ 147-150 \\ 153\end{array}$

153-154

154-157

$158-183$

$183-186$

187-196

196-201

$$
0-6
$$

6- 12

12- 22

$22-27$

27- 60

$60-91$

$91-130$

$130-156$

$156-165$

$165-172$

$172-190$

190-194

194-206

$0-3$

3- 9

10- 26

26- 28

$28-60$

$60-70$

$70-75$

$75-87$

$87-99$

$100-114$

fil1

boulder

clay

sand

sand

NHW 563

gravel

hardpan

boulder

NHW 564

boulder topsoil and sand

sand, brown

cobbles and sand

sand and gravel

sand and gravel

hardpan and sand

gravel and sand, fine

lost circulation

grave1, medium

clay and clay sand

gravel, large

clay, hardpan

gravel and water

soil and loam

grave1, coarse

hardpan - cobbles

hardpan

sand, clayey, brown

clay, gray

sand, clayey, gray

sand, fine, brown

sand, fine, gray, water

clay, gray

loam - topsoil

loam, sand and stone

hardpan gravel

sand and gravel, medium

sand and gravel, finer

clay sand, gray brown

clay sand, gray

sand, fine, hard, brown

gravel and sand, medium
NHW 565

$0-6$

6- 14

$14-30$

$30-45$

$45-50$

$50-65$

$65-108$

108

$0-1$

2- 9

$10-45$

$46-50$

$50-67$

$67-70$

$71-85$

$85-105$

$106-114$

0- 1

$1-9$

9- 11

$11-18$

$18-19$

$19-27$

$27-28$

$29-60$

$60-75$

$75-93$

$93-98$

$99-125$

$125-128$

$129-140$

$140-164$

grave 1

sand

clay

topsoil small boulders

sand, gray hardpan

clay, gray

gravel, brown

sand, fine brown

NHW 566

sand hardpan, brown

sand and gravel, brown

gravel and sand, medium

sand and grave1, coarse

boulders and cobbles

grave1, coarse

sand and gravel, medium

gravel and sand, medium to

NHW 567

topsoil

loam sand

boulders

hardpan sand

boulders

hardpan sand, gravel seams

boulder

gravel, cobbles, hardpacked

(lost mud)

sand and gravel

sand, dark brown

sand and gravel (lost mud)

clay, gray

gravel, small, sandy

clay hardpan

sand and gravel 


Depth below
land surface the sediment
(in feet)

\begin{tabular}{ll}
\hline Depth below & Description of \\
land surface the sediment \\
(in feet)
\end{tabular}

NHW 569

$\begin{aligned} 0-9 & \text { sand and gravel } \\ 10-17 & \text { hardpan } \\ 17-24 & \text { gravel } \\ 24-25 & \text { boulder } \\ 26-30 & \text { hardpan } \\ 30-45 & \text { clay } \\ 45-47 & \text { gravel } \\ 47-57 & \text { clay } \\ 57-59 & \text { gravel, medium } \\ 59-64 & \text { clay } \\ 65-90 & \text { gravel, medium } \\ 90-92 & \text { gravel } \\ 92-105 & \text { gravel, sandy } \\ 105-109 & \text { gravel, medium } \\ 110-123 & \text { hardpan, gravel } \\ 124-133 & \text { sand, fine, hard, gray } \\ 133-144 & \text { sand, brown } \\ 145-147 & \text { gravel, medium } \\ 148-150 & \text { clay } \\ 150-161 & \text { sand and gravel }\end{aligned}$

NHW 570

$0-11$

$11-16$

16

$16-28$

$28-42$

$42-60$

$60-83$

83- 92

$92-96$

$96-135$

$135-165$

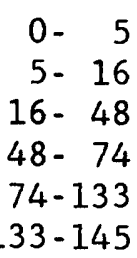

sand, silty, gray

gravel

cobbles, boulder

cobbles, hardpan

cobbles and gravel

sand, fine, brown

sand, dark brown

clay, gray

gravel and hardpan

sand and gravel

sand, fine, brown

NHW 572

sand

loam, sandy

sand, clayey

sand, gravelly

clay and hardpan

sand, gray, clayey
NHW 573

0 - 0.5 topsoil

0.5- 9 sand, yellow

9- 18 sand, brown

19- 32 clay sand, gray

32- 42 sand, gray brown

42- 46 gravel, medium, orange

47- 56 cobbles and boulders

57- 60 sand and gravel

60- 76 hardpan gravel, clay seams

76- 83 clay hardpan, sandy, gray

83- 90 clay seams - gray hardpan

91- 94 hardpan gravel, gray

94-106 clay hardpan, gray

106-120 clay seams, gray

$120-131$

$131-136$

$137-140$

$141-150$

$150-152$

$153-160$

$161-170$

$170-172$

$173-178$

$178-180$

$180-190$

$0-15$

15- 60

$61-78$

$78-81$

$82-84$

$84-93$

$94-125$

$126-136$

$138-148$

$150-182$

183-184

$184-194$ clay hardpan

clay and gravel seams

sand

sand, grave1, hardpan

sand, white

clay hardpan

clay and clay sand

gravel, sandy

clay

boulder

sand and gravel

NHW 574

sand, yellow

sand, brown

sand and gravel

hardpan sand

boulder

sand and gravel, water

sand

clay sand

sand and gravel, quartz

hardpan, sandy

boulder

sand and gravel 
Table 2.--Lithologic logs of selected wells and test borings--Continued

\begin{tabular}{ll}
\hline Depth below & Description of \\
land surface & the sediment \\
(in feet) & \\
\hline
\end{tabular}

\begin{tabular}{ll}
\hline Depth below & Description of \\
land surface the sediment \\
(in feet)
\end{tabular}

\begin{tabular}{|c|c|}
\hline & NHW 575 \\
\hline $0-5$ & sand, brown \\
\hline $5-8$ & sand, gray \\
\hline $8-21$ & hardpan, gray \\
\hline $21-27$ & gravel and hardpan, brown \\
\hline $27-32$ & clay, sandy \\
\hline $32-54$ & sand, brown \\
\hline $54-63$ & hardpan, sandy, gray \\
\hline $63-110$ & gravel, sandy, brown \\
\hline $110-124$ & hardpan, sandy, gray \\
\hline $124-134$ & sand, fine, silty, brown \\
\hline $134-140$ & clay, blue \\
\hline $140-144$ & sand, clayey \\
\hline $144-160$ & clay \\
\hline $160-176$ & hardpan \\
\hline $176-194$ & hardpan, sand and gravel \\
\hline & NHW 576 \\
\hline $0-7$ & silt, loamy \\
\hline $7-18$ & clay \\
\hline $18-23$ & hardpan \\
\hline $23-34$ & sand, fine, brown \\
\hline & $\begin{array}{l}\text { sand and gravel, brown, } \\
\text { water }\end{array}$ \\
\hline & NHW 577 \\
\hline $0-16$ & hardpan, gravelly \\
\hline & boulder \\
\hline $16-37$ & grave1 and sand \\
\hline 17 & boulder \\
\hline 24 & boulder \\
\hline $37-46$ & sand, packed \\
\hline $46-51$ & hardpan, brown \\
\hline 51 & boulder \\
\hline $51-60$ & cobbles and gravel \\
\hline $60-71$ & sand, hardpan \\
\hline $71-93$ & sand and gravel, dirty \\
\hline & NHW 578 \\
\hline $150-170$ & $\begin{array}{l}\text { hardpan, some light gray- } \\
\text { white sandy clay }\end{array}$ \\
\hline $220-230$ & $\begin{array}{l}\text { clay, sandy, light gray, } \\
\text { with soft black organic } \\
\text { grains, } \\
\text { occasional cobble }\end{array}$ \\
\hline
\end{tabular}

NHW 583

$0-38$

$38-87$

$87-105$

$105-127$

$127-144$

$0-4$

4- 32

$32-45$

$0-9$

$9-36$

$36-42$

$0-2$

$2-5$

$5-15$

$15-20$

$20-21$

$21-27$

$27-29$

$29-34$

$34-35$

$55-39$

$39-40$

$40-61$

$61-73$

$73-84$

0- 11

$11-60$

$60-70$

$70-78$

$78-93$

$93-96$

$96-136$ gravel

sand, fine, brown

clay, gray

gravel hardpan and sand, gray

grave1, clayey

NHW 601

loam

sand and grave 1

gravel, sandy

NHW 604

sand, silty

sand and silty clay

sand and gravel

NHW 606

topsoil

loam sand

clay sand

hardpan gravel, sandy

boulder

hardpan gravel

boulder

sand and gravel

boulder

sand and gravel

clay, yellow

gravel, hard (mud loss)

gravel, hard, sand and

grave1

sand and gravel

NHW 607

grave 1

sand, brown (boulders at 8

$\mathrm{ft}$ and $34 \mathrm{ft}$ )

sand, caving

sand, clayey

gravel

cobbles, large

sand 
Table 2.--Lithologic logs of selected wells and test borings--Continued

\begin{tabular}{ll}
\hline Depth below & Description of \\
land surface & the sediment \\
(in feet) & \\
\hline
\end{tabular}

\begin{tabular}{cl}
\hline Depth below & Description of \\
land surface & the sediment \\
(in feet) &
\end{tabular}

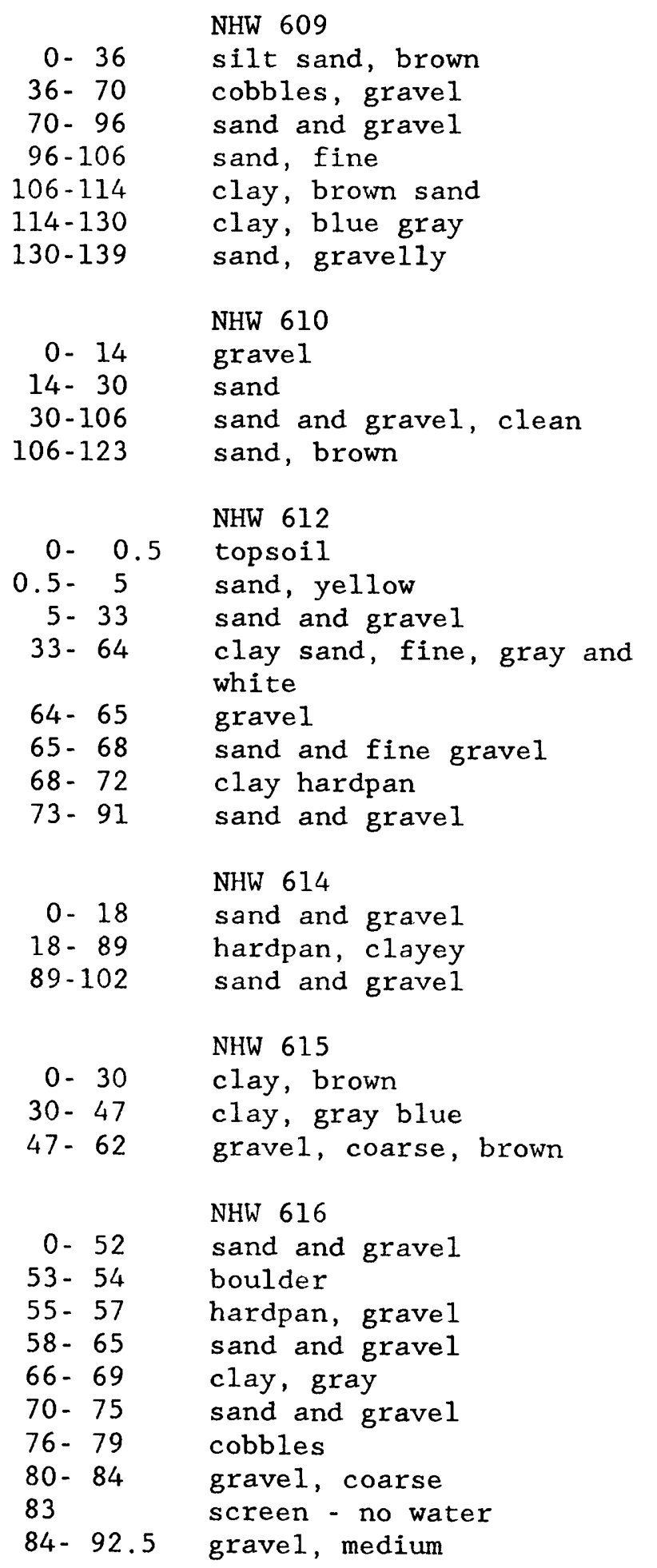

$0-18$
$18-19$
$19-21$
$21-35$
$35-64$
$64-78$
$78-82$
$82-90$

$0-7$

$7-44$

44- 78

$78-93$

0- 1

1- 4

5- 15

$15-48$

$48-67$

$67-68$

$68-82$

$82-83$

$83-85$
$0-4$
4- 9
9- 23
$23-43$
$43-53$
53

$0-22$

$22-42$

$42-51$

$51-56$

0- 30

$30-75$

$75-95$

\section{NHW 617}

grave1, coarse

grave1, finer

cobbles - boulder

grave1, sandy

sand, dirty, brown

clay

sand and gravel

water in gravel

NHW 619

sand

grave1, brown

sand and gravel

sand, gravel, water

NHW 622

topsoil, loam

gravel and clay

sand and gravel

hardpan and clay, sand seams

gravel, coarse, hard

boulder

sand and gravel

sand

clay sand, gray

NHW 623

loam, sandy

grave 1

sand, dirty

gravel, fine

hardpan

gravel, coarse

NHW 625

sand and gravel

clay, brown

grave1, sand

gravel, coarse

NHW 626

sand

clay

sand 
Table 2.--Iithologic logs of selected wells and test borings--Continued

Depth below Description of
land surface the sediment
(in feet)

\begin{tabular}{ll}
\hline Depth below & Description of \\
land surface & the sediment \\
(in feet) &
\end{tabular}

NHW 627

$\begin{array}{cl}0-2 & \begin{array}{l}\text { soil, black } \\ \text { till, silty, sandy, dry, } \\ \text { grayish brown } \\ \text { till, silty, sandy, dry, } \\ \text { brownish gray }\end{array} \\ 16-23.5 \quad \begin{array}{l}\text { till, sandy, silty, wet, } \\ \text { gray } \\ \text { gravel and fine to very } \\ \text { coarse sand, trace very fine } \\ \text { sand }\end{array}\end{array}$

NHW 628

0- 2 sand, silt and gravel

2- 25 till, silty, sandy, gravelly, medium gray, with several layers or zones of brownish gray

NHW 629

$\begin{array}{lr}0- & 2 \\ 2- & 4 \\ 4- & 35\end{array}$

soil, organic

silt

till, silty, sandy, hard, medium gray; more gravelly at depth with some light brown to light gray and dark gray

NHW 630

$\begin{aligned} & 0-1 \begin{array}{l}\text { soil, organic, and brown } \\ \text { silt } \\ \text { silt, firm, light brown, } \\ \text { probably not layered }\end{array} \\ & \text { silt, sandy, with occasional } \\ & \text { pebbles }\end{aligned}$

\section{$0-6$ \\ $6-26$ \\ $26-39$ \\ $39-55$ \\ $55-63$}

$63-64$

0- 2

2- 9

9- 10

10- 13

13

$15-19$

19

$19-21.5$

$0-41$

41- 64

$64-75$

$75-118$

$118-122$

44- 54

$54-70$

$70-79$

$79-83$

$83-89$

$89-129$
$0-44$

NHW 631

sand, very fine to medium

sand, very fine, clean

sand, very fine to fine

till, stony

sand, very coarse to medium, and granules, rusty, clayey (not till)

sand, very fine and silt

\section{NHW 632}

\section{soil, light brown}

silt, grayish brown, very

fine to fine sand and subrounded pebble gravel

\section{cobble}

silt, pebbly, sandy

boulder; moved $5 \mathrm{ft}$ south; 0

$\mathrm{ft}$ to $15 \mathrm{ft}$ same

sand, very fine to very

coarse, silty, gravelly;

water at $16 \mathrm{ft}$

cobble

till, sandy, gravelly, gray

NHW 633

gravel

clay and sand, gray

sand, fine, brown

silt, clayey, brown

gravel, clayey, brown

NHW 635

sand and gravel

gravel - cobbles

sand and gravel

gravel, coarse, cobbles

gravel, coarse

cobbles and gravel

gravel, coarse

NHW 640

0- 1 soil

1- 3 clay

3 - 5 clay and sand

$5-54.4$ clay

55.4-63.4 sand 
Table 2.--Lithologic logs of selected wells and test borings--Continued

\begin{tabular}{ll}
\hline Depth below & Description of \\
land surface & the sediment \\
(in feet) & \\
\hline
\end{tabular}

0. 34

34- 51

$51-63$

$63-70$

$70-74$

$74-76$

$76-83$

$83-100$

$100-105$

105-107

107-110

110-112

$112-127$

$127-131$

$131-134$

0- 11

$11-28$

$28-36$

$36-46$

$46-57$

$57-65$

$65-68$

$68-87$

$87-92$

92

0- 30

$30-70$

$70-95$

$95-109$

110
NHW 650

sand, brown

sand, gray

clay, sandy, gray, brown

sand, gray brown

sand and grave1, brown

clay, red

gravel, dirty, red brown

hardpan, sandy, gray

hardpan, red-gray

hardpan, gray

hardpan, clayey, white and red

clay, white, little sand

sand, fine, white

sand and gravel, quartz, white

hardpan and sand, white

NHW 651

grave 1

sand

grave1

sand, brown

hardpan, gray

sand, brown

clay sand, brown

clay hardpan, gray

sand and grave1, brown

water bearing

NHW 652

grave1, sandy

clay, sandy

sand, fine, brown (bad

boulder at $82 \mathrm{ft}$ )

sand, white, and little

grave 1

clay, white

\begin{tabular}{cl}
\hline $\begin{array}{c}\text { Depth below } \\
\text { land surface } \\
\text { (in feet) }\end{array}$ & $\begin{array}{l}\text { Description of } \\
\text { the sediment }\end{array}$ \\
\hline
\end{tabular}

NHW 654

0 - 3 loam

3- 13 kaolin

13- 26 sand, white

26- 34 sand, gray

34- 48 hardpan

48- 55 gravelly

$55-70$ sand

70- 99 clay sand, silty, brown

99-103 sand, beach

$103-120$

$120-124$

clay hardpan, gray

gravel, water

NHW 655

0 - 9 gravel

9- 32 sand and grave1

$32-35$

$35-43$

$43-65$

$65-74$

$74-90$

$90-101$

$101-109$

109

gravel

sand

hardpan, sand

sand, brown

gravel

grave1, sand, water

sand, quartz, white

clay, white

NHW 656

0- 2 sand, 1oamy

2- 4 sand, white

4 boulder

4- 12 sand, brown

12- 14 kaolin

14- 28 sand, quartz, white

$28-30$

$30-34$

$34-35$

$35-38$

$38-56$

$56-58$

$58-70$

$70-85$ kaolin

sand, white

clay sand

clay and hardpan

gravel and sand

cobbles

sand and gravel

gravel, large, and sand 
Table 2.--Lithologic logs of selected wells and test borings--Continued

Depth below Description of
land surface the sediment
(in feet)

Depth below Description of
land surface the sediment
(in feet)

\section{NHW 657}

$\begin{array}{rr}0- & 4 \\ 4- & 10 \\ 10- & 12 \\ 12- & 16 \\ 16-22 \\ 22-27 \\ 27-39 \\ 39-48 \\ 48-55 \\ 55-66 \\ 66-78 \\ 78\end{array}$

NHW 658

hardpan

soil, clayey, brown

kaolin, white

sand and gravel, white

sand, brown

gravel

sand

grave1, coarse

hardpan and clay, gray

hardpan, soft, gray

sand, fine, brown

gravel and sand

muck

4- 11 sand

$11-20$

$20-23$

$23-26$

0- 4

4- 8

$8-22$

$22-30$

$30-42$

$42-55$

$55-60$

$60-78$

$78-108$

$108-111$

\section{$0-4$}

4- 6

$6-12$

$12-15$

$15-42$

$42-43$

$43-49$

$49-50$

$50-55$

$55-57$

$57-83$

$83-96$ clay sand, brown

cobbles and gravel, hardpan

grave1, water

\section{NHW 660}

sand and grave1, loamy

gravel, sand and cobbles

hardpan

clay, sandy

hardpan, sandy

hardpan, clayey

clay, gray

sand, very fine, gray

sand and clay

sand

NHW 661

sand, brown

boulder

sand and grave 1

cobbles

sand and gravel

boulder

sand and gravel

boulder

sand and gravel

cobbles

hardpan, sandy

clay, fine, sandy, with water
NHW 662

$0-20$
$20-28$
$28-46$
$46-58$

grave 1

sand

hardpan, clayey

gravel and sand; boulder at $49 \mathrm{ft}$

NHW 664

$0-7$

$7-11$

$11-14$

$14-18$

$18-25$

$25-31$

$31-105$

$105-132$

132

0- 11

$11-18$

$18-35$

$35-53$

$0-27$

$27-28$

$28-38$

$38-48$

$48-75$

94

95- 107

$107-112$

$112-117$

$0-19$

$19-22$

$22-28$

$28-34$

$34-49$

$49-59$

$59-63$

$63-85$

$85-100$

$100-111$ sand

grave1

mar1

gravel, fine

grave1, coarse, gray,

shells, and cobbles

grave1, coarse, brown, and shells

hardpan, gray

sand, clayey

gravel and fine sand

NHW 665

sand

grave 1, coarse

gravel, medium, brown

sand and gravel-water

NHW 667

sand, brown

boulder

sand and gravel

gravel and sand

sand, clayey

cobble, brown-no water

clay

sand, clayey and water

sand, clay, gravel

NHW 668

sand and gravel, dirty

sand and gravel

cobbles and gravel

hardpan, brown

sand and gravel

gravel and cobbles, coarse

sand, gray, gravel, quartz

grave 1

clay and sand

sand gravel 
Table 2.--Lithologic logs of selected wells and test borings--Continued

\begin{tabular}{ll}
\hline Depth below & Description of \\
land surface & the sediment \\
(in feet) & \\
\hline
\end{tabular}

NHW 669

0- 45 no record

45- 66 sand and hardpan

66- 95 sand and water

95-100 sand, brown, gray

$\begin{array}{cl} & \text { NHW } 670 \\ 0-2 & \text { soil } \\ 2-70 & \text { gravel, coarse, and sand } \\ 70-110.2 & \text { gravel and sand }\end{array}$

$\begin{array}{rrl}0- & \text { NHW } 673 \\ 1- & \text { topsoil } \\ 6-9 & \text { sand } \\ 9-16 & \text { grave1 } \\ 16-18 & \text { sand, clayey } \\ 18-30 & \text { boulder } \\ 30-40 & \text { grave1 - cobbles } \\ 41-44 & \text { cobbles and large gravel } \\ 45 & & \text { grave1 } \\ .5-46.5 & \text { boulder }\end{array}$

$5.5-46.5$ boulder

$\begin{array}{rll} & & \text { NHW } 674 \\ 0- & 5 & \text { grave1, dirty } \\ 5- & 13 & \text { sand, loamy } \\ 13-31 & \text { gravel, coarse } \\ 31-48 & \text { sand and grave1 } \\ 48-55 & \text { sand } \\ 55-64 & \text { grave1 and sand } \\ & \\ 0- & \text { NHW } 675 \\ 6-18 & \text { sand clay } \\ 18-36 & \text { boulders and grave1 } \\ & \end{array}$

$\begin{array}{ll}\text { Depth below } & \text { Description of } \\ \text { land surface } & \text { the sediment } \\ \text { (in feet) } & \end{array}$

$\begin{array}{rrl} & & \text { NHW } 676 \\ 0- & 4 & \text { sand } \\ 4- & 6 & \text { boulder } \\ 6- & 9 & \text { sand } \\ 9- & 10 & \text { boulder } \\ 10-15 & \text { sand and gravel }\end{array}$

15- 21 hardpan gravel, yellow

22- 25 cobbles

26- 81 hardpan, gravel

$82-83$ cobbles, gravel

83-110 hardpan, gravel

110-118 clay sand, brown

118-123 gravel, sandy

$123-136$

$136-147$

$147-150$

$151-157$

$157-159$

$160-185$

clay sand, hard, gray

clay, gray

sand

clay

clay sand

sand and gravel

NHW 677

0 - 2 topsoil

2- 8 sand

8- 15 sand and boulders

$15-55$

$55-57$

$58-62$

$62-85$

$85-100$

$100-108$

$108-124$

sand and gravel - cobbles

hardpan

sand, gravel

hardpan and gray clay

hardpan, gray

clay, gray

sand and gravel

NHW 678

$0-28$

$28-35$

grave1, silt

$33-40$

$40-79$

$79-110$

cobbles

gravel, coarse

grave1, sandy

grave1

$110-116$

$116-124$

$124-131$ gravel, very coarse

hardpan

sand, cobbles, gravel 
Table 2.--Lithologic logs of selected wells and test borings--Continued

\begin{tabular}{ll}
\hline Depth below & Description of \\
land surface & the sediment \\
(in feet) &
\end{tabular}

$\begin{array}{ll}\text { Depth below } & \text { Description of } \\ \text { land surface the sediment } \\ \text { (in feet) }\end{array}$

\begin{tabular}{|c|c|c|}
\hline & NHW 679 & \\
\hline $0-20$ & grave1, cobbles & $\begin{array}{r}0-26 \\
26-40\end{array}$ \\
\hline $20-32$ & gravel & $26-40$ \\
\hline $32-35$ & boulder, cobbles & $40-62$ \\
\hline $35-39$ & gravel, coarse & $62-76$ \\
\hline $39-56$ & grave1 & $76-92$ \\
\hline $56-72$ & gravel and sand & $92-109$ \\
\hline $72-86$ & sand, dirty & $109-122$ \\
\hline & boulder & \\
\hline $86-109$ & sand and gravel & \\
\hline $109-120$ & sand, coarse & $119-156$ \\
\hline $120-128$ & sand, clayey & $156-165$ \\
\hline $128-135$ & hardpan, clayey & $165-168$ \\
\hline $135-145$ & sand, clayey & \\
\hline $145-166$ & clay, gray & \\
\hline $166-167$ & clay hardpan, gray & \\
\hline $167-173$ & grave1 and sand & \\
\hline 174 & hardpan & 8 - \\
\hline $174-183$ & hardpan & $9-22$ \\
\hline $175-176$ & boulder & $22-28$ \\
\hline $183-186$ & hardpan, gravelly & $28-37$ \\
\hline $186-189$ & gravel, sandy & $37-46$ \\
\hline & & $46-$ \\
\hline & NHW 680 & \\
\hline $0-10$ & cobbles and boulders & \\
\hline $10-28$ & gravel, coarse & \\
\hline $28-38$ & grave1 & $8-17$ \\
\hline $38-78$ & sand, clayey & $17-3$ \\
\hline $78-90$ & clay, blue gray & $30-4$ \\
\hline $90-93$ & hardpan, cobbles & $43-5$ \\
\hline $93-106$ & gravel & $58-7$ \\
\hline & & $78-9$ \\
\hline & NHW 682 & $93-9$ \\
\hline $0-3$ & loam, sand & $95-10$ \\
\hline 4- 9 & hardpan, gravel & 103 \\
\hline $10-26$ & cobbles and gravel & $103-11$ \\
\hline $27-48$ & sand and gravel & $110-11$ \\
\hline $49-59$ & sand, fine, hard & $116-11$ \\
\hline $60-85$ & sand and gravel, fine & $118-12$ \\
\hline $86-90$ & clay sand & $126-13$ \\
\hline $91-160$ & sand, fine & 132 \\
\hline $161-190$ & clay sand & $132-15$ \\
\hline $191-211$ & gravel, medium & $150-17$ \\
\hline $212-232$ & sand and hardpan & $172-17$ \\
\hline & & 177 \\
\hline & & $177-1$ \\
\hline
\end{tabular}

\section{NHW 684}

hardpan, sandy, gray

grave1, coarse - hardpan

grave1, coarse, brown

hardpan, sandy, gray

sand and clay, black

clay, blue

gravel

deepened; log continued

below

gravel, coarse

sand and gravel

gravel and fine sand

16 in. gravel at bottom

NHW 685

silt and sand

gravel, dirty

hardpan, gray

sand, silty, brown

gravel, coarse, brown

sand, brown

sand and gravel

NHW 686

loamy and grave1

sand, clayey

clay, blue

sand, quartz, white

gravel, brown

sand and gravel, high iron

clay hardpan

sand, fine

hardpan

lens grave 1

sand, fine

hardpan

sand, fine, gray

sand, fine, brown

sand and gravel

hardpan lens

sand, fine, gray brown

hardpan and clay

hardpan

no water

gravel material 
Table 2.--Lithologic logs of selected wells and test borings--Continued

Depth below
land surface
(in feet)

\begin{tabular}{cl}
\hline Depth below & Description of \\
land surface & the sediment \\
(in feet) & \\
\hline
\end{tabular}

NHW 687

0. 52 sand, gravel, cobbles, boulders

$53-56$

$57-83$

boulder

$86-88$

$88-123$

$123-126$

$127-135$

sand and gravel

boulder

hardpan, sandy, gray

clay, gray

gravel and water

NHW 688

0 - 2 sand

2- 3 boulder

$3-4$ sand

4- 5 boulder

5- 7 cobbles

$7-12$

12- 13

$13-17$

$17-18$

$18-40$

40

gravel, sandy

boulder

gravel, sandy

boulder

gravel, sandy

boulder

$40-42$

$42-50$

$50-55$

$55-62$

$62-71$

$71-95$

95-106

$106-107$

$107-118$

$118-120$

$120-130$

$0-0.5$

$0.5-4$

4- 12

12- 24

$24-28$

29- 40

$41-55$

$56-105$

$105-120$

$121-128$ gravel, sandy

hardpan

sand and gravel, gray

hardpan

clay sand

clay sand and gravel

hardpan

boulder

hardpan

clay

sand and gravel

NHW 689

sand, yellow

hardpan gravel, dark

clay hardpan gravel

sand and gravel

sand, hardpacked

sand and gravel

sand, brown

clay sand, gray topsoil

sand and gravel
NHW 691

$0-14$

14- 20

$20-24$

$24-28$

$28-46$

$46-64$

$64-86$

$86-103$

$103-120$

$120-206$

206-216

0. 24

$24-40$

$40-54$

$54-62$

$62-70$

$70-74$

$74-88$

$88-102$

$102-140$

$140-164$

$164-180$

$180-184$

$184-248$

$248-254$

0 - 1

2- 16

$17-30$

$33-37$

$38-43$

44- 50

$50-68$

$68-74$

$75-92$

$93-108$

$110-140$

$141-143$

$144+$

sand

loam

clay soil and brown clay

sand and gravel

hardpan, gray

sand, gray

gravel, brown

hardpan, clayey

gravel and sand

silt, clayey

sand, clayey, gray

clay, gray

grave 1 and sand

\section{NHW 692}

boulders and gravel

sand, brown

sand, black

sand, brown

clay, brown

gravel and sand, brown

gravel, brown

gravel, coarse

sand, very fine, brown

sand, fine, gray

sand, gray

clay, gray

sand and gray gravel

NHW 693

clay sand, yellow

sand, gray brown

clay sand

grave1, medium

sand and grave 1

hardpan, gray, and gravel

gravel and hardpan

sand and gravel

sand and clay seams

gravel, fine

hardpan and sand 
Table 2.--Lithologic logs of selected wells and test borings--Continued

\begin{tabular}{ll}
\hline Depth below & Description of \\
land surface & the sediment \\
(in feet) & \\
\hline
\end{tabular}

$\begin{array}{cl}\text { Depth below } & \text { Description of } \\ \text { land surface } & \text { the sediment } \\ \text { (in feet) } & \end{array}$

NHW 695

$0-9$
$9-18$
18
$18-24$
24
$24-28$
$28-65$
$65-78$
$78-96$
$96-105$

$0-1$

$1-4$

5- 9

9- 15

$15-22$

$\begin{array}{rr}0- & 4 \\ 4- & 62 \\ 62-80 \\ 80-116 \\ 116-120 \\ 120-125 \\ 125-132 \\ 132-142 \\ 142-156 \\ 156-161\end{array}$

$0-60$

$60-84$

$84-123$

$123-143$

$$
\begin{array}{rr}
0- & 1 \\
1- & 4 \\
4- & 30 \\
30- & 45 \\
45- & 60
\end{array}
$$

boulder

cobbles

boulder

NHW 696

topsoil

NHW 698

fill

gravel

sand

NHW 699

coarse

NHW 700

topsoil soil, clayey

clay hardpan

grave1, coarse

sand, clayey, brown

hardpan, gray

clay, gray

sand and grave1, clayey

sand, yellow

gravel, medium

hardpan, gray, and gravel

gravel and cobbles

gravel, coarse

clay, very fine sandy

sand, clayey

clay, brown

sand, clayey, brown, water

sand, fine, clayey, brown

sand gravel, rusty

gravel and sand, medium

sand, brown

gravel, cobbly, some sand

sand, medium, brown

grave1, sandy, gray

sand and grave1

sand and grave1, medium

sand and gravel, medium,

cobbles
NHW 703

sand

grave1

silt, brown clayey

grave1, brown

boulder

grave1

hardpan

$48-67$
$67-85$

$85-98$

$98-136$

$136-170$

$170-178$

$178-188$

188

hardpan, gray sandy

sand, fine brown

clay, brown

sand, clayey, gray

gravel, clayey, gray

grave1, sand

NHW 704

$0-86$

$86-98$

$98-110$

$110-112$

112

$112-130$

$130-136$

$136-186$

185

$0-$

$122-155$

244

$0-21$

$21-32$

$32-80$

80

grave 1

hardpan

gravel and sand

cobbles

boulder trouble

gravel, coarse

cobbles

gravel and cobbles

water

NHW 706

boulders and cobbles

like rock

sand and gravel

NHW 708

grave1

clay, brown

sand and grave1

gravel

NHW 709

$0-18$

$18-54$

54-108 soil and gravel - coarse

gravel

sand and gravel 
Table 2.--Lithologic logs of selected wells and test borings--Continued

\begin{tabular}{ll}
\hline Depth below & Description of \\
land surface the sediment \\
(in feet)
\end{tabular}

$\begin{array}{ll}\text { Depth below } & \text { Description of } \\ \text { land surface } & \text { the sediment } \\ \text { (in feet) } & \end{array}$

\begin{tabular}{|c|c|}
\hline & NHW 710 \\
\hline $0-6$ & sand \\
\hline $6-35$ & sand and grave 1 \\
\hline $35-45$ & sand and small cobbles \\
\hline $\begin{array}{l}45-65 \\
65\end{array}$ & $\begin{array}{l}\text { sand, gravel, hardpan } \\
\text { boulder }\end{array}$ \\
\hline $66-98$ & $\begin{array}{l}\text { sand and grave1, hardpan } \\
\text { seams }\end{array}$ \\
\hline $\begin{array}{l}98-104 \\
104\end{array}$ & $\begin{array}{l}\text { grave } 1 \text { and cobbles, clean } \\
\text { boulder }\end{array}$ \\
\hline $106-115$ & sand and gravel \\
\hline $116-118$ & boulder \\
\hline $118-134$ & sand and cobbles \\
\hline $134-139$ & clay \\
\hline $139-141$ & sand and gravel \\
\hline $141-144$ & clay \\
\hline $145-164$ & sand and gravel \\
\hline & NHW 712 \\
\hline $0-100$ & grave1, coarse, and sand \\
\hline $100-153$ & sand, medium \\
\hline $153-154$ & clay, gray \\
\hline $154-165$ & sand, fine \\
\hline & NHW 714 \\
\hline $0-2$ & sand and gravel fill \\
\hline $2-\quad 2.5$ & concrete slab \\
\hline $3-5$ & sand loam, brown \\
\hline $5-9$ & grave1, dark brown \\
\hline $10-18$ & sand and gravel \\
\hline $18-22$ & boulder \\
\hline $23-35$ & $\begin{array}{l}\text { sand, light brown, and } \\
\text { grave1 }\end{array}$ \\
\hline $35-50$ & $\begin{array}{l}\text { sand and grave1, medium, and } \\
\text { clay seams }\end{array}$ \\
\hline $51-58$ & $\begin{array}{l}\text { sand and gravel, medium to } \\
\text { coarse, and clay seams }\end{array}$ \\
\hline $59-63$ & hardpan, gray \\
\hline $\begin{array}{l}63-65 \\
65-66\end{array}$ & $\begin{array}{l}\text { clay hardpan } \\
\text { boulder }\end{array}$ \\
\hline $66-70$ & $\begin{array}{l}\text { hardpan grave1, gray, and } \\
\text { clay seams }\end{array}$ \\
\hline $70-79$ & gravel and hardpan layers \\
\hline $79-82$ & clay sand \\
\hline $83-97$ & hardpan grave1, gray \\
\hline $98-105$ & sand and gravel \\
\hline $106-107$ & cobbles and gravel \\
\hline
\end{tabular}

$\begin{array}{rr}0- & 5 \\ 6- & 12 \\ 12- & 56 \\ 57- & 65 \\ 66-86\end{array}$

$0-5$

$5-18$

$18-23$

$23-42$

$43-46$

$46-91$

$91-100$

$101-124$

$$
\begin{array}{rr}
0- & 2 \\
2- & 25 \\
25- & 40 \\
40- & 41 \\
41- & 80 \\
80- & 83 \\
83- & 92
\end{array}
$$

$0-1$

$1-10$

$10-14$

$14-18$

$18-24$

$24-33$

$33-63$

$0-22$

$22-38$

$38-80$

$80-120$

$120-135$

$135-172$

$172-188$

188-193

193-198
NHW 715

grave1, fill

clay, brown

sand and gravel

clay hardpan

gravel and sand, quartz

NHW 716

fill - sand and cobbles

sand, dark brown

sand and hardpan seams

hardpan and gravel seams

clay, gray

sand, gray white

clay, dark gray

gravel, small to medium

\section{NHW 717}

topsoil

clay and hardpan

sand and gravel

boulder

sand and gravel

cobbles

sand and gravel

NHW 718

soil

clay, brown

sand, coarse

clay, gray (hardpan)

clay and sand

sand

sand, coarse

NHW 720

sand and silt

sand, clayey

grave 1

sand

sand, fine

clay sand, fine

hardpan, very hard, gray

grave1, clayey

grave 1 
Table 2.--Lithologic logs of selected wells and test borings--Continued

\begin{tabular}{ll}
\hline Depth below & Description of \\
land surface & the sediment \\
(in feet) &
\end{tabular}

\begin{tabular}{cl}
\hline Depth below & Description of \\
land surface & the sediment \\
(in feet) &
\end{tabular}

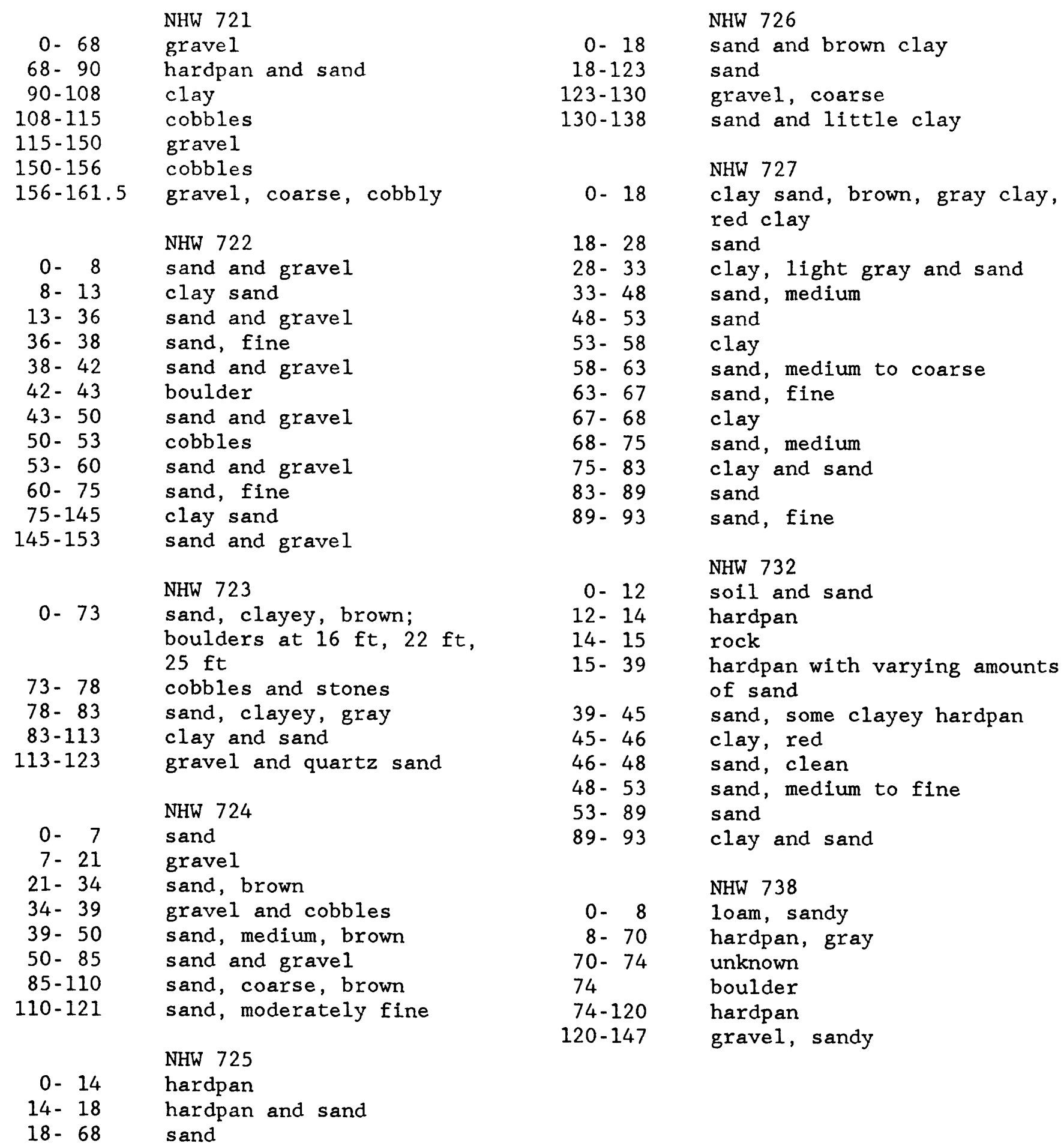


Table 2.--Lithologic logs of selected wells and test borings--Continued

\begin{tabular}{ll}
\hline Depth below & Description of \\
land surface & the sediment \\
(in feet) & \\
\hline
\end{tabular}

NHW 741

$0-3$

4- 5

5- 20

$21-26$

$26-31$

$34-36$

$36-45$

$45-70$

$70-76$

$76-86$

87-104

$0-9$

9- 23

$23-34$

$34-40$

$40+$

0- 7

7- 40

$40-42$

$42-50$

$50-61$

$61-68$

$68-78$

$78-100$

$100-103$

$103-110$

$0-5$

$5-16$

$16-34$

$34-56$

56- 102

102-112

$0-7$

$7-15$

$15-40$

$40-61$

$61-68$

$68-73$ sand, brown

gravel, fine

sand, dark brown

clay, brown sand

gravel, medium

boulder

gravel and clay seams

hardpan gravel, gray

clay sand, gray

hardpan, clay gravel seams

sand and gravel

NHW 742

gravel

gravel, sandy

sand, gray

hardpan - gray sand

fine sandy material, brown

NHW 743

soil, clayey

clay, gravelly, brown

gravelly

clay

sand, clayey

gravelly with hardpan

clay, sandy

clay

hardpan, clayey

gravelly

NHW 744

loam, black

cobbles and grave1

clay sand, silty

sand, clayey, brown

hardpan, clayey

sand and gravel, brown

NHW 745

sand, loamy

clay, brown sand

hardpan

clay hardpan

hardpan, gravelly

sand, gravelly

\begin{tabular}{ll}
\hline Depth below & Description of \\
land surface & the sediment \\
(in feet) &
\end{tabular}

NHW 751

0- 32

sand, silty

$32-36$

$36-45$

$45-60$

60

$60-78$

$78-88$

$88-98$

$98-102$

$0-5$

5- 19

20- 27

$28-30$

$31-38$

$40-60$

$61-90$

91- 104

$105-130$

$131-135$

$135-160$

$160-182$

$183-192$

192-209

209-212

$212-214$

214-216

$216-220$

$220-221$

$222-231$

$232-237$

$238-246$

0- 10

$10-47$ grave1, clayey

gravel, brown

gravel, sandy, brown

water

sand, brownish black

hardpan

hardpan, sandy

sand and gravel

NHW 752

clay sand, gray

sand, brown

clay, gray

hardpan, brown

hardpan - gravel seams

hardpan

gravel, hard

clay sand, gray

gravel, medium hard

hardpan, gray

clay sand, gray

clay, gray

hardpan

sand and gravel

clay

sand and grave 1

clay sand

cobble

hardpan and sand

sand, gray

sand and grave1, quartz, gray

NHW 756

soil, sandy

gravel sand and grave 1 
Table 2.--Lithologic logs of selected wells and test borings--Continued

\begin{tabular}{ll}
\hline Depth below & Description of \\
land surface & the sediment \\
(in feet) &
\end{tabular}

NHW 760

0- 14

14- 38

38

$38-46$

$46-58$

$58-80$

$80-142$

0- 32

$32-63$

$63-76$

$76-79$

$79-120$

120-160

0- 41

41- 51

$51-52$

$52-58$

$58-68$

$68-69$

$69-88$

$88-90$

90- 94

94- 96

$96-140$

$140-156$

$156-161$

$161-164$

$0-10$

10- 35

$35-50$

$50-75$

$75-79$

$79-83$

0 - 8

$8-53$

53- 70

$70-95$

95- 143

\section{gravel}

gravel and cobbles, coarse

bad boulder

cobbles - gravel

sand and gravel

gravel

sand and gravel

NHW 761

sand

sand and gravel

grave 1

boulder

grave1

sand and gravel

NHW 762

sand, brown

sand and gravel

boulder

gravel

hardpan

boulder

grave 1

clay sand

clay

clay sand

sand and gravel

sand, brown - water

sand, fine, gray

sand, brown - no water

NHW 765

sand and cobbles

gravel, coarse

gravel and sand, coarse

grave1, medium

sand

gravel

NHW 766

sand, loamy

sand and grave 1

grave1, coarse, cobbly

gravel

sand and gravel

$\begin{array}{ll}\text { Depth below } & \text { Description of } \\ \text { land surface } & \text { the sediment } \\ \text { (in feet) } & \end{array}$

NHW 767

0- 12

grave 1

$12-38$

$38-63$

$63-74$

$74-99$

$99-110$

110 -

sand, clayey

clay sand, silty, gray

clay, gray

sand, brown

hardpan, brown

grave1

NHW 768

0 - 7 grave 1

7- 36 clay silt, brown

$36-39$

$39-56$

$56-62$

$62-104$

$104-124$

$124-131$

clay, gray

clay silt, brown

clay, gray

clay sand, brown

clay, gray

grave1

NHW 769

$\begin{array}{rr}0- & 8 \\ 8- & 38 \\ 38-76 \\ 76-94 \\ 94-100\end{array}$

sand, silty

clay sand, brown

sand, fine, brown

clay, gray

grave1

NHW 771

$0-1$

$1-33$

soil

$33-43$

boulders

$43-57$

sand

unknown

$57-63$

$63-83$

clay and sand

sand and clay

83-101

sand 
Table 2.--Lithologic logs of selected wells and test borings--Continued




Table 3.- Total monthly precipitation at selected stations (801-811) from October 1988 to December 1990 [ $\cdots$, no data available; values are in inches]

\begin{tabular}{|c|c|c|c|c|c|c|c|c|c|c|}
\hline \multicolumn{11}{|l|}{ STATION } \\
\hline NUMBER & OCT 88 & NOV 88 & DEC 88 & JAN 89 & FEB 89 & MAR 89 & APR 89 & MAY 89 & JUN 89 & JUL 89 \\
\hline 801 & $\cdots$ & 10.19 & 1.84 & 1.77 & 2.41 & 3.66 & 5.30 & $\cdots$ & .. & $\cdots$ \\
\hline 802 & $-\cdot$ & 7.54 & 1.01 & 1.36 & 2.66 & 3.86 & 3.71 & 3.54 & 6.11 & 7.21 \\
\hline 803 & -- & 8.72 & 1.63 & 1.38 & 3.66 & 4.07 & 4.20 & -. & -. & $\cdots$ \\
\hline 804 & $\cdots$ & 7.87 & 1.65 & 1.72 & 2.54 & 4.25 & 4.27 & 3.88 & 5.40 & 6.88 \\
\hline 805 & $\cdots$ & 12.56 & 2.48 & 1.50 & 2.73 & 4.25 & 4.29 & 3.73 & 5.70 & 6.53 \\
\hline 806 & $-\cdot$ & -. & 1.53 & 1.63 & 2.28 & 5.76 & 4.21 & 4.06 & 6.26 & 8.71 \\
\hline 807 & 2.67 & 8.03 & 1.38 & 1.57 & 1.56 & 3.34 & 4.99 & 6.38 & 4.51 & 5.82 \\
\hline 808 & $\cdots$ & 7.58 & 2.36 & 1.88 & 2.86 & 5.07 & 5.07 & 4.39 & 5.33 & 7.12 \\
\hline $1^{809}$ & $\cdots$ & $-\cdot$ & -- & 1.34 & 2.40 & 4.45 & 4.47 & 4.52 & 5.19 & 7.35 \\
\hline${ }^{1} 810$ & 2.58 & 9.11 & 1.23 & 1.48 & $\therefore$ & -- & $-\cdot$ & 3.87 & 5.64 & 7.09 \\
\hline 811 & -- & 7.50 & 1.41 & 1.74 & 2.73 & 4.02 & 4.88 & 3.50 & 5.86 & 7.55 \\
\hline
\end{tabular}

\begin{tabular}{|c|c|c|c|c|c|c|c|c|c|c|}
\hline \multicolumn{11}{|l|}{ STATION } \\
\hline NUMBER & AUG 89 & SEP 89 & OCT 89 & NOV 89 & DEC 89 & JAN 90 & FEB 90 & MAR 90 & APR 90 & MAY 90 \\
\hline 801 & $\cdots$ & $\cdots$ & $\cdots$ & -- & $\cdots$ & -. & $\cdots$ & $\cdots$ & $\cdots$ & $\cdots$ \\
\hline 802 & 3.72 & $\cdots$ & $\cdots$ & -. & - & $\cdots$ & $\cdots$ & 3.92 & 4.89 & 2.77 \\
\hline 803 & -. & $\cdots$ & - & -. & $\cdots$ & .. & $\cdots$ & $\therefore$ & $-\cdot$ & $\cdots$ \\
\hline 804 & $\cdots$ & $\ldots$ & $\cdots$ & - & $\cdots$ & -. & - & $\cdots$ & $-\cdot$ & -- \\
\hline 805 & 3.91 & 4.19 & 6.68 & 4.31 & 3.34 & 5.12 & 2.79 & 1.68 & 5.89 & 6.15 \\
\hline 806 & 3.94 & -- & 5.00 & -. & $-\cdot$ & - & -. & - & - & $\cdots$ \\
\hline 807 & 3.36 & 4.15 & 4.31 & 4.51 & 1.96 & 4.54 & $\cdots$ & 1.86 & 5.04 & 5.33 \\
\hline 808 & 4.12 & 3.60 & 5.01 & 4.75 & 2.81 & 5.27 & 3.11 & 1.84 & 5.41 & 6.81 \\
\hline 809 & 2.75 & 3.95 & 6.45 & 3.25 & 3.60 & -. & .. & .. & - & $\cdots$ \\
\hline ' 810 & 3.30 & 3.89 & 6.00 & 4.06 & 1.69 & 6.17 & 2.39 & 1.33 & 5.82 & 5.70 \\
\hline 811 & 3.46 & 5.21 & 4.17 & 5.85 & 2.01 & 4.49 & 2.85 & 1.75 & 4.20 & 7.12 \\
\hline
\end{tabular}

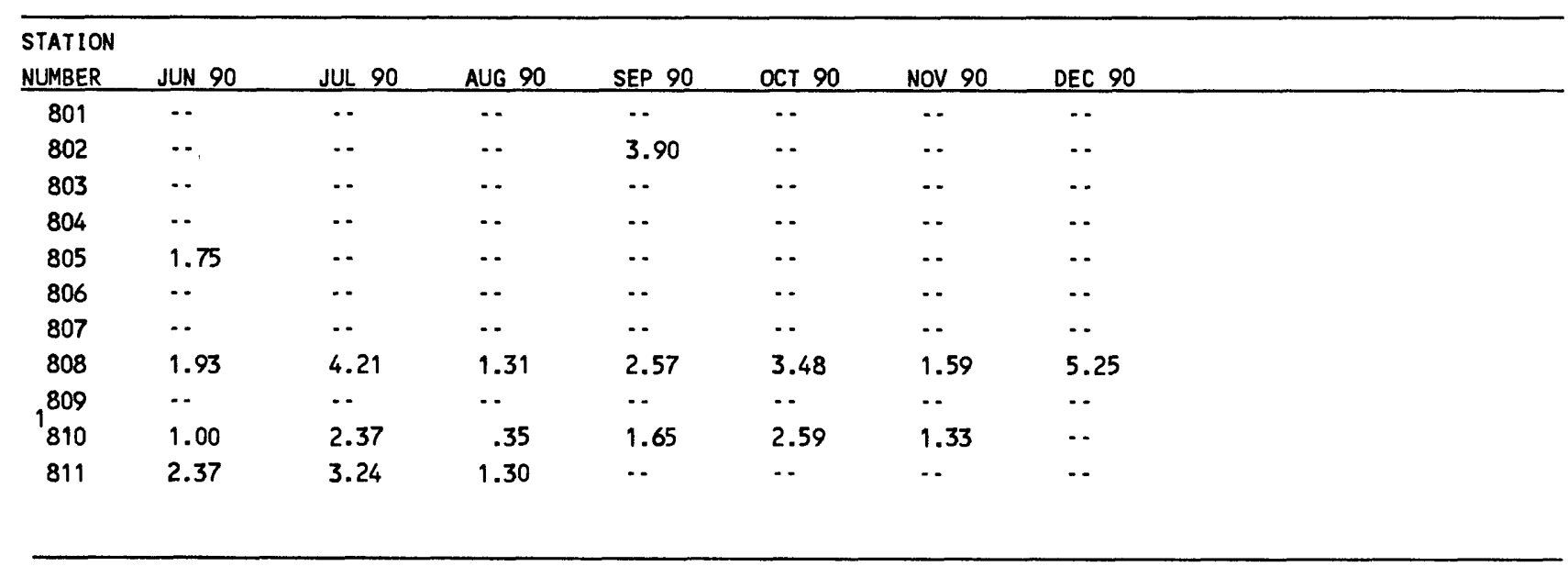

\footnotetext{
${ }^{1}$ Station 810 is Block Island State Airport weather station
} 


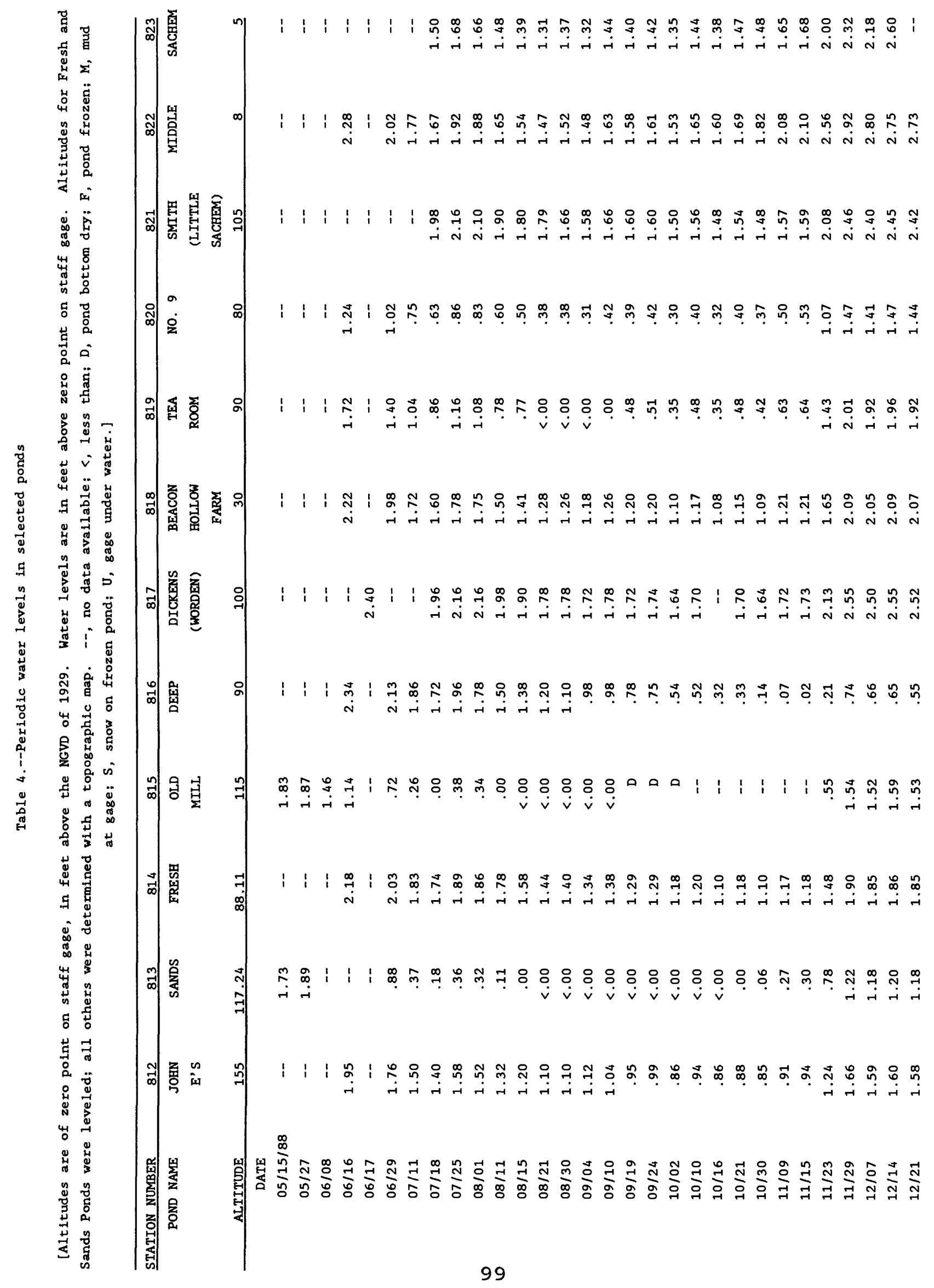




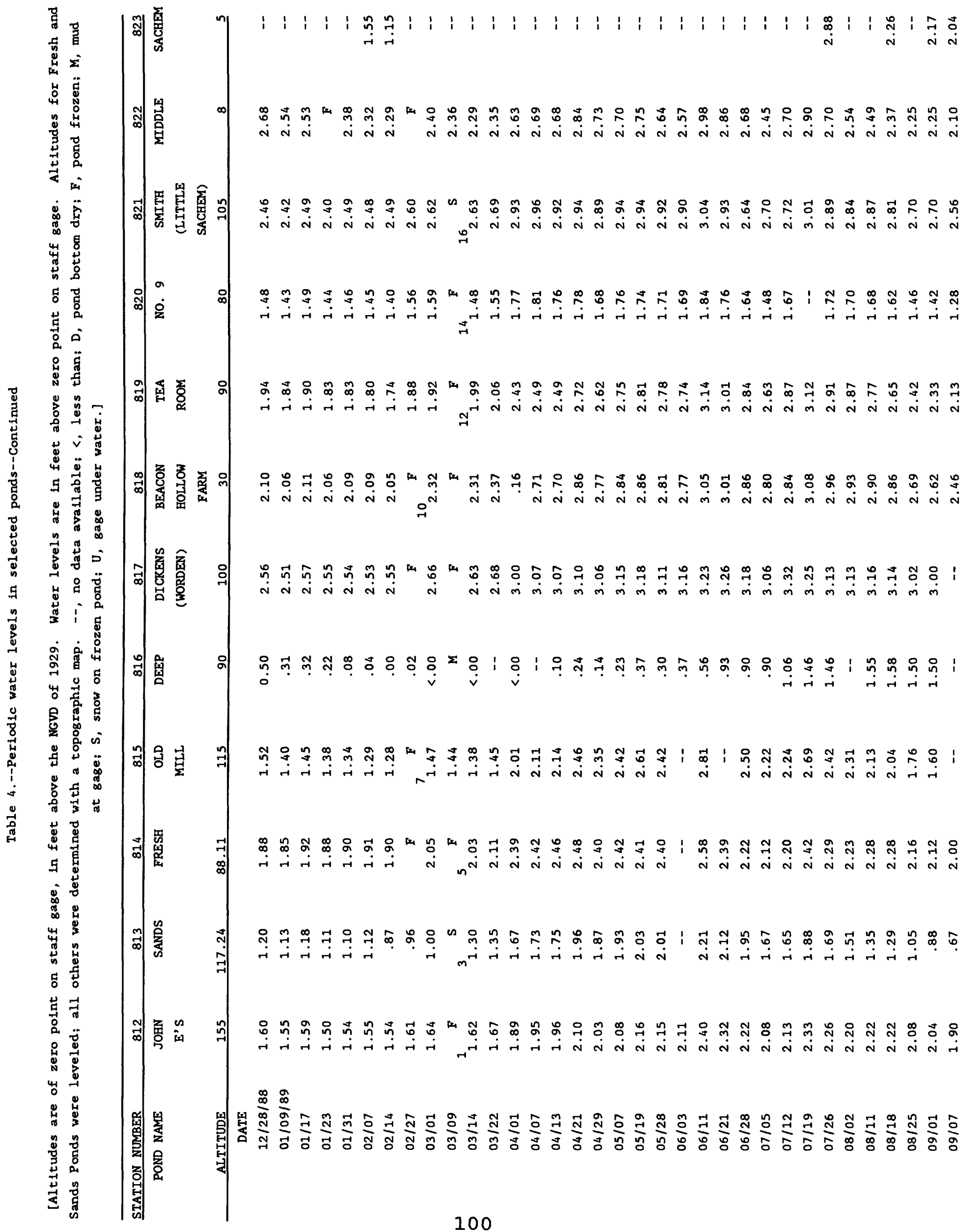




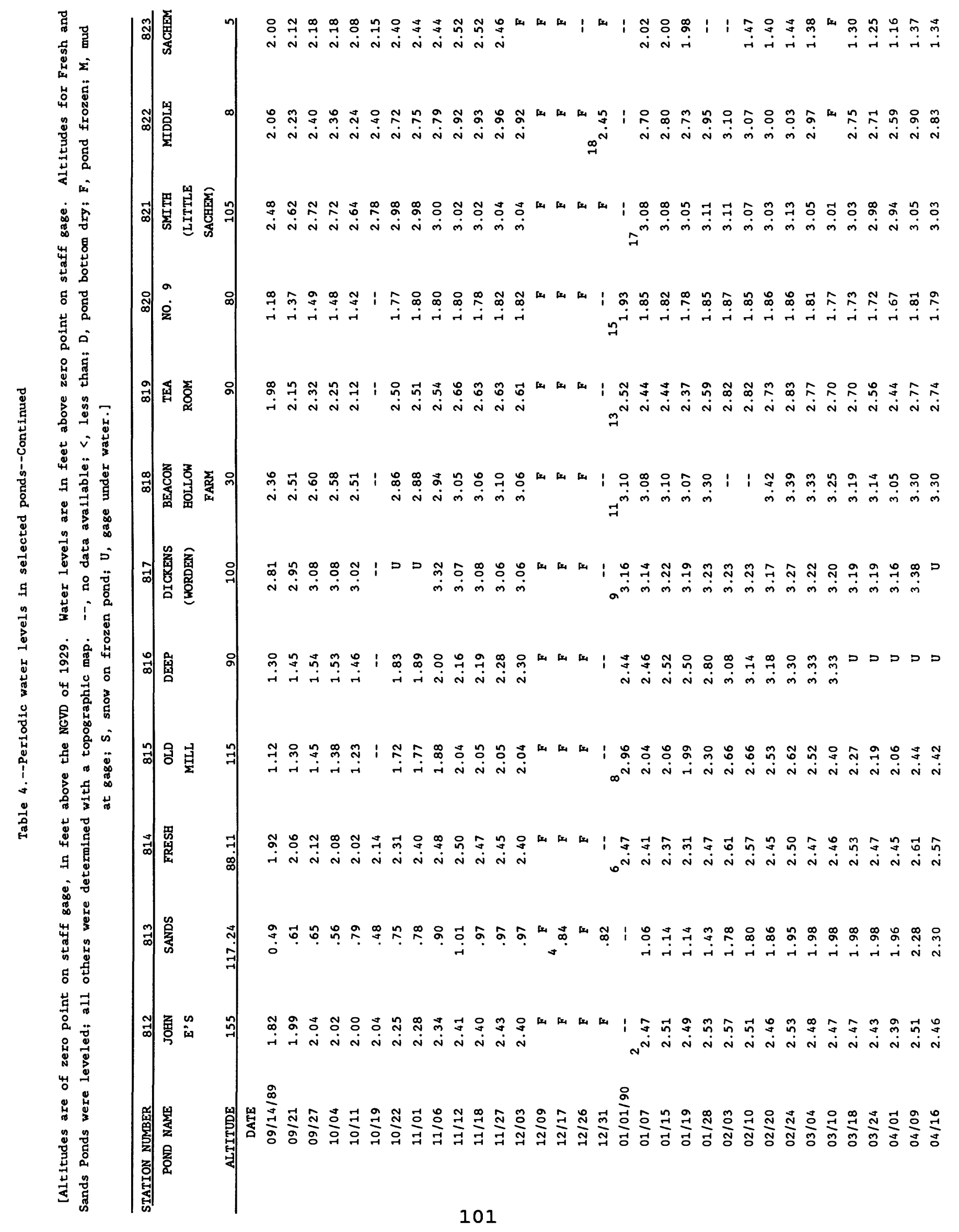




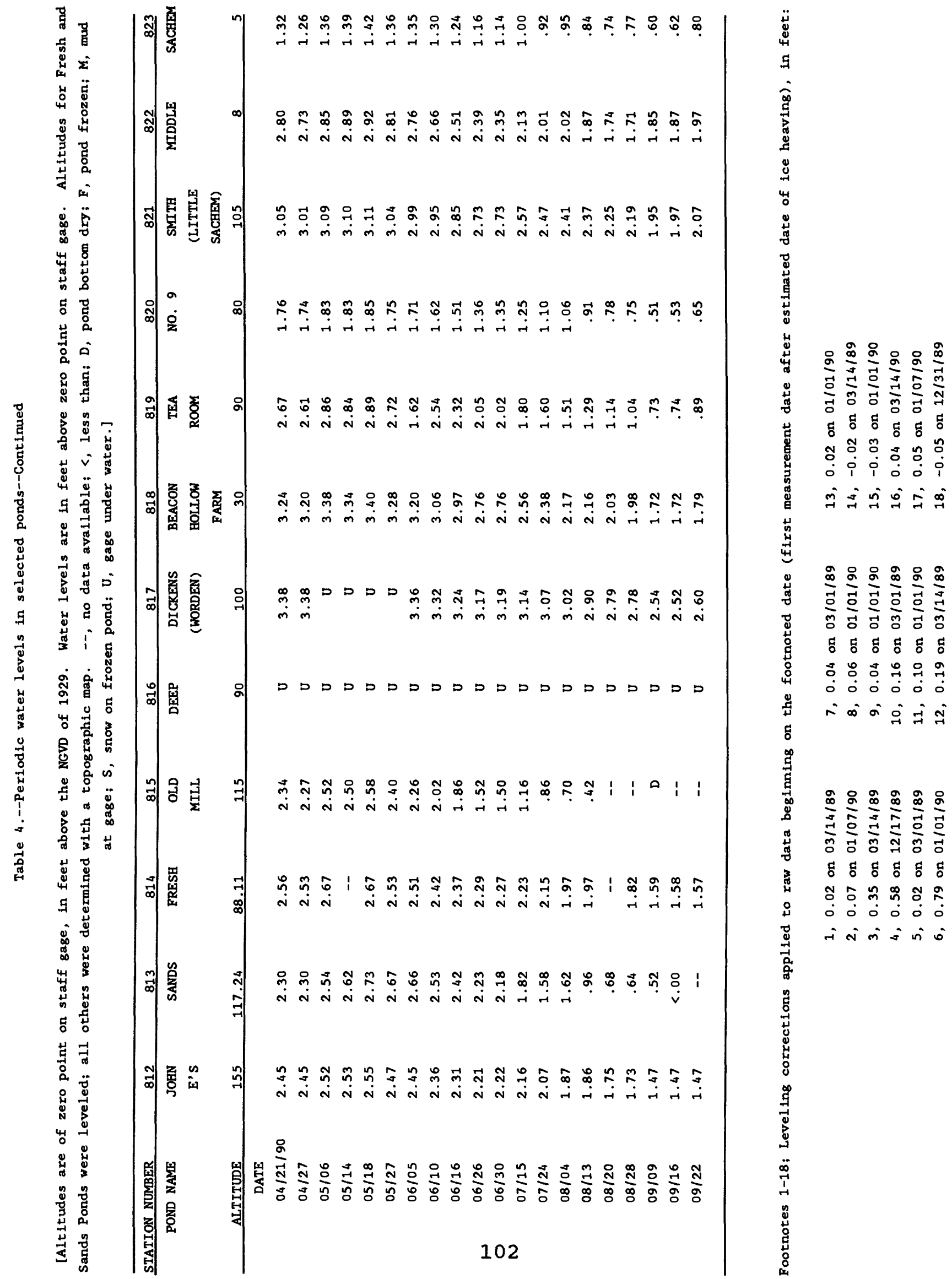


Table 5. - Periodic water levels in selected wells

[Water levels are in feet below land surface; Measurement methods: $E$, estimated, method unknown, to nearest ft; $R$, reported to nearest $\mathrm{ft}$; $S$, steel tape, measured to nearest $0.01 \mathrm{ft}$; $T$, electric tape, measured to nearest $0.1 \mathrm{ft}$; $Z$, acoustic meter, measured to nearest $0.1 \mathrm{ft}]$

\begin{tabular}{lclc}
\hline & Date & & Method \\
& water & Water & water \\
Station & level & level & level \\
number & measured & (feet) & measured \\
\hline
\end{tabular}

\begin{tabular}{llll}
\hline & Date & & Method \\
& water & Water & water \\
level & level & level \\
Station & measured & (feet) & measured \\
number & & & \\
\hline
\end{tabular}

\begin{tabular}{|c|c|c|c|c|}
\hline \multirow[t]{3}{*}{ NHW } & 11 & $06-12-62$ & 6.43 & s \\
\hline & & $08 \cdot 31-62$ & 5.64 & $\mathbf{s}$ \\
\hline & & $09 \cdot 24 \cdot 62$ & 5.76 & s \\
\hline \multirow[t]{2}{*}{ NHW } & 17 & $07-02-62$ & 2.70 & s \\
\hline & & $09-01-62$ & 2.71 & $\mathbf{s}$ \\
\hline \multirow[t]{11}{*}{ NHW } & 33 & $08-16-62$ & 21.02 & $\mathbf{s}$ \\
\hline & & $08-31-62$ & 21.71 & $\mathbf{s}$ \\
\hline & & $10 \cdot 08 \cdot 62$ & 22.52 & $\mathbf{s}$ \\
\hline & & $05-23-63$ & 21.32 & $\mathbf{s}$ \\
\hline & & $05-24-63$ & 21.89 & $\mathbf{s}$ \\
\hline & & $03 \cdot 30 \cdot 89$ & 21.4 & $T$ \\
\hline & & $05 \cdot 09 \cdot 89$ & 18.8 & $T$ \\
\hline & & $06-09-89$ & 17.3 & $\mathbf{T}$ \\
\hline & & $06-21-89$ & 16.74 & $\mathbf{s}$ \\
\hline & & $08-10-90$ & 17.67 & $\mathbf{s}$ \\
\hline & & $06-14-91$ & 18.00 & s \\
\hline \multirow[t]{8}{*}{ NHW } & 34 & $08 \cdot 16 \cdot 62$ & 35.41 & $s$ \\
\hline & & $08-31-62$ & 35.86 & $\mathbf{s}$ \\
\hline & & $09-06-62$ & 35.79 & $\mathbf{s}$ \\
\hline & & $10-08-62$ & 36.37 & $\mathbf{s}$ \\
\hline & & $07-27-79$ & 30 & $\mathbf{R}$ \\
\hline & & $06-21-89$ & 32.34 & $\mathbf{s}$ \\
\hline & & $08-10-90$ & 31.29 & $\mathbf{s}$ \\
\hline & & $06-14-91$ & 32.8 & $T$ \\
\hline \multirow[t]{2}{*}{ NHW } & 49 & $05-04 \cdot 88$ & 14.65 & $\mathbf{s}$ \\
\hline & & $06-20-89$ & 14.48 & $s$ \\
\hline \multirow[t]{4}{*}{ NHW } & 62 & $06 \cdot 21 \cdot 60$ & 113.32 & s \\
\hline & & $07-22 \cdot 62$ & 109.35 & $\mathbf{s}$ \\
\hline & & $08-25 \cdot 62$ & 108.55 & $\mathbf{s}$ \\
\hline & & $09-18-62$ & 108.67 & s \\
\hline \multirow[t]{2}{*}{ NHW } & 72 & $09-18-62$ & 122.85 & $\mathbf{s}$ \\
\hline & & $10 \cdot 02 \cdot 62$ & 122.85 & $\mathbf{s}$ \\
\hline \multirow[t]{4}{*}{ NHW } & 75 & $06 \cdot 16-62$ & 7.07 & $\mathbf{s}$ \\
\hline & & $10 \cdot 06-62$ & 11.56 & s \\
\hline & & $04-14-88$ & 4.40 & $\mathbf{s}$ \\
\hline & & $05-16-88$ & 5.60 & $\mathbf{s}$ \\
\hline \multirow[t]{3}{*}{ NHW } & 76 & $06-20-62$ & 8.62 & $\mathbf{s}$ \\
\hline & & $10-06-62$ & 12.83 & $\mathbf{s}$ \\
\hline & & $05 \cdot 25 \cdot 63$ & 9.20 & s \\
\hline \multirow[t]{5}{*}{ NHW } & 80 & $06-23 \cdot 62$ & 11.02 & s \\
\hline & & $07-02-62$ & 11.94 & $\mathbf{s}$ \\
\hline & & $08-13-62$ & 12.02 & s \\
\hline & & $09-06-62$ & 13.03 & $\mathbf{s}$ \\
\hline & & $03-11-88$ & 10.18 & $\mathbf{s}$ \\
\hline
\end{tabular}

\begin{tabular}{|c|c|c|c|c|}
\hline \multirow[t]{2}{*}{ NHW } & 80 & $06-19-89$ & 9.62 & $\mathbf{s}$ \\
\hline & & $06-21-89$ & 9.82 & $\mathbf{s}$ \\
\hline \multirow[t]{2}{*}{ NHW } & 83 & $06-25-62$ & 9.35 & s \\
\hline & & $08-18-62$ & 11 & $E$ \\
\hline \multirow[t]{3}{*}{ NHW } & 91 & $06-27-62$ & 20.35 & s \\
\hline & & $08-20-62$ & 20.62 & $\mathbf{s}$ \\
\hline & & $03-18-88$ & 21.0 & $\mathbf{s}$ \\
\hline \multirow[t]{2}{*}{ NHW } & 92 & $06 \cdot 27-62$ & 3.08 & $\mathbf{s}$ \\
\hline & & $09-03.62$ & 6.40 & $\mathbf{s}$ \\
\hline \multirow[t]{2}{*}{ NHW } & 93 & $06-27-62$ & 4.44 & $\mathbf{s}$ \\
\hline & & $09-02-62$ & 6.82 & $\mathbf{S}$ \\
\hline \multirow[t]{4}{*}{ NHW } & 94 & $06-27-62$ & 7.49 & $\mathbf{S}$ \\
\hline & & $08-13-62$ & 10.62 & $\mathbf{s}$ \\
\hline & & $09-03-62$ & 11.34 & $\mathbf{s}$ \\
\hline & & $06-22 \cdot 88$ & 7.47 & $\mathbf{s}$ \\
\hline \multirow[t]{3}{*}{ NHW } & 96 & $06-29 \cdot 62$ & 1.57 & $\mathbf{s}$ \\
\hline & & $09-01-62$ & 2.40 & s \\
\hline & & $05-04-88$ & 0.72 & $\mathbf{s}$ \\
\hline \multirow[t]{3}{*}{ NHW } & 97 & $06-29 \cdot 62$ & 8.37 & $\mathbf{s}$ \\
\hline & & $08-13-62$ & 10.62 & $\mathbf{S}$ \\
\hline & & $09.03-62$ & 10.98 & $\mathbf{s}$ \\
\hline \multirow[t]{2}{*}{ NHW } & 99 & $08-31-62$ & 13.31 & $\mathbf{s}$ \\
\hline & & $09-20-62$ & 13.50 & $\mathbf{S}$ \\
\hline \multirow[t]{3}{*}{ NHW } & 101 & $06-30-62$ & 2.02 & $\mathbf{s}$ \\
\hline & & $08-13-62$ & 2.22 & $\mathbf{s}$ \\
\hline & & $09 \cdot 03 \cdot 62$ & 3.12 & $\mathbf{s}$ \\
\hline \multirow[t]{2}{*}{ NHW } & 102 & $06-30-62$ & 9.40 & $\mathbf{S}$ \\
\hline & & $09-03-62$ & 14.06 & $\mathbf{s}$ \\
\hline \multirow[t]{2}{*}{ NHW } & 103 & $09-22-62$ & 54.65 & $s$ \\
\hline & & $10-08 \cdot 62$ & 54.58 & $\mathbf{s}$ \\
\hline \multirow[t]{5}{*}{ NHW } & 104 & $07-01-62$ & 3.17 & $\mathbf{s}$ \\
\hline & & $09 \cdot 06-62$ & 3.71 & $\mathbf{s}$ \\
\hline & & $03 \cdot 24-88$ & 1.8 & $\mathbf{s}$ \\
\hline & & $06 \cdot 19 \cdot 89$ & 1.79 & $\mathbf{s}$ \\
\hline & & $06-21-89$ & 1.69 & $\mathbf{s}$ \\
\hline \multirow[t]{3}{*}{ NHW } & 107 & $07-02-62$ & 6.40 & $\mathbf{S}$ \\
\hline & & $09.06 \cdot 62$ & 6.81 & $\mathbf{s}$ \\
\hline & & $05-03-88$ & 5.46 & $\mathbf{s}$ \\
\hline \multirow[t]{2}{*}{ NHW } & 108 & $07 \cdot 02-62$ & 8.40 & s \\
\hline & & $08-16 \cdot 62$ & 9.50 & $\mathbf{s}$ \\
\hline \multirow[t]{3}{*}{ NHW } & 110 & $07-02 \cdot 62$ & 13.37 & $\mathbf{s}$ \\
\hline & & 09.02 .62 & 20.86 & $\mathbf{s}$ \\
\hline & & $10-06-62$ & 22.36 & s \\
\hline NHW & 111 & $08-22-62$ & 83.82 & $\mathbf{s}$ \\
\hline
\end{tabular}


Table 5.--Periodic water levels in selected wells--Continued

[Water levels are in feet below land surface; Measurement methods: $E$, estimated to nearest ft; $R$, reported to nearest $\mathrm{ft}$; $S$, steel tape, measured to nearest $0.01 \mathrm{ft}$; $T$, electric tape, measured to nearest $0.1 \mathrm{ft}$; $Z$, acoustic meter, measured to nearest $0.1 \mathrm{ft}]$

\begin{tabular}{|c|c|c|c|c|}
\hline \multicolumn{2}{|c|}{$\begin{array}{l}\text { Station } \\
\text { number }\end{array}$} & $\begin{array}{l}\text { Date } \\
\text { water } \\
\text { level } \\
\text { measured }\end{array}$ & $\begin{array}{l}\text { Water } \\
\text { level } \\
\text { (feet) }\end{array}$ & $\begin{array}{l}\text { Method } \\
\text { water } \\
\text { level } \\
\text { measured }\end{array}$ \\
\hline \multirow[t]{2}{*}{ NHW } & 111 & $09-21-62$ & 84.05 & $\mathbf{s}$ \\
\hline & & $10-09-62$ & 83.57 & $\mathbf{S}$ \\
\hline \multirow[t]{7}{*}{ NHW } & 113 & $07-03-62$ & 5.61 & $\mathbf{s}$ \\
\hline & & $07-11-62$ & 6.03 & $\mathbf{S}$ \\
\hline & & $07-26-62$ & 6.40 & $\mathbf{s}$ \\
\hline & & $08-13-62$ & 6.57 & $\mathbf{S}$ \\
\hline & & $09 \cdot 02.62$ & 6.94 & $\mathbf{s}$ \\
\hline & & $09-20-62$ & 8.08 & $\mathbf{S}$ \\
\hline & & $03-10-88$ & 2.40 & $\mathbf{s}$ \\
\hline \multirow[t]{4}{*}{ NHW } & 114 & $07-03-62$ & 3.74 & $\mathbf{s}$ \\
\hline & & $09 \cdot 06 \cdot 62$ & 4.63 & $\mathbf{S}$ \\
\hline & & $06-13-89$ & 0.86 & $\mathbf{S}$ \\
\hline & & $06-21-89$ & 1.35 & $\mathbf{s}$ \\
\hline \multirow[t]{2}{*}{ NHW } & 115 & $07-03-62$ & 1.94 & $s$ \\
\hline & & $09-06-62$ & 2.31 & $S$ \\
\hline \multirow[t]{3}{*}{ NHW } & 116 & $07-04-62$ & 5.03 & $S$ \\
\hline & & $08-13-62$ & 6.00 & $\mathbf{S}$ \\
\hline & & $09-01-62$ & 4.73 & $\mathbf{s}$ \\
\hline \multirow[t]{2}{*}{ NHW } & 117 & $07-04-62$ & 3.18 & $\mathbf{s}$ \\
\hline & & $08-13-62$ & 3.16 & $S$ \\
\hline \multirow[t]{2}{*}{ NHW } & 118 & $10-02 \cdot 62$ & 100.84 & $s$ \\
\hline & & $06-13-91$ & 102.3 & $T$ \\
\hline \multirow[t]{3}{*}{ NHW } & 119 & $07-04-62$ & 7.95 & $s$ \\
\hline & & $09-01.62$ & 13.75 & $\mathbf{S}$ \\
\hline & & $06-21-89$ & 3.55 & $\mathbf{s}$ \\
\hline \multirow[t]{6}{*}{ NHW } & 121 & $07-04-62$ & 5.91 & $s$ \\
\hline & & $08-05-62$ & 6.73 & $\mathbf{s}$ \\
\hline & & $09-02-62$ & 6.11 & $\mathbf{s}$ \\
\hline & & $06-15 \cdot 88$ & 5.76 & $\mathbf{s}$ \\
\hline & & $06-07-89$ & 5.30 & $\mathbf{s}$ \\
\hline & & $06-21-89$ & 4.65 & $s$ \\
\hline \multirow[t]{2}{*}{ NHW } & 122 & $07-05-62$ & 2.70 & $s$ \\
\hline & & $09 \cdot 06 \cdot 62$ & 3.25 & $\mathbf{s}$ \\
\hline \multirow[t]{6}{*}{ NHW } & 125 & $07 \cdot 05 \cdot 62$ & 10.78 & S \\
\hline & & $07-11 \cdot 62$ & 10.87 & $s$ \\
\hline & & $07-23-62$ & 10.96 & $s$ \\
\hline & & $09-01-62$ & 10.72 & $s$ \\
\hline & & $10-02-62$ & 10.88 & $s$ \\
\hline & & $03-30-88$ & 7.3 & $\mathbf{s}$ \\
\hline \multirow[t]{2}{*}{ NHW } & 126 & $07-05 \cdot 62$ & 21.57 & $\mathbf{s}$ \\
\hline & & $09-01-62$ & 22.33 & $\mathbf{s}$ \\
\hline \multirow[t]{3}{*}{ NHW } & 131 & $07-06-62$ & 3.13 & $\mathbf{S}$ \\
\hline & & $09-01-62$ & 3.12 & $s$ \\
\hline & & $03-30-88$ & 2.3 & $T$ \\
\hline \multirow[t]{2}{*}{ NHW } & 135 & $07-06-62$ & 9.85 & $\mathbf{s}$ \\
\hline & & $09-01-62$ & 10.62 & $\mathbf{s}$ \\
\hline
\end{tabular}

\begin{tabular}{|c|c|c|c|c|}
\hline \multicolumn{2}{|c|}{$\begin{array}{l}\text { Station } \\
\text { number }\end{array}$} & \multirow{2}{*}{$\begin{array}{c}\begin{array}{c}\text { Date } \\
\text { water } \\
\text { level } \\
\text { measured }\end{array} \\
07-09-62\end{array}$} & \multirow{2}{*}{$\begin{array}{l}\begin{array}{l}\text { Water } \\
\text { level } \\
\text { (feet) }\end{array} \\
40.00\end{array}$} & \multirow{2}{*}{$\begin{array}{c}\text { Method } \\
\text { water } \\
\text { level } \\
\text { measured } \\
\text { s }\end{array}$} \\
\hline NHW & 136 & & & \\
\hline & & $09-10-62$ & 31.33 & $s$ \\
\hline \multirow[t]{3}{*}{ NHW } & 137 & $07-07-62$ & 14.12 & $\mathbf{s}$ \\
\hline & & $09-01-62$ & 15.81 & $s$ \\
\hline & & $10-06-62$ & 15.92 & $\mathbf{s}$ \\
\hline \multirow[t]{2}{*}{ NHW } & 138 & $07-07-62$ & 3.60 & $\mathbf{s}$ \\
\hline & & $09 \cdot 06 \cdot 62$ & 2.87 & $s$ \\
\hline \multirow[t]{2}{*}{ NHW } & 144 & $07-09-62$ & 17.54 & $s$ \\
\hline & & $09 \cdot 05-62$ & 18.01 & $\mathbf{s}$ \\
\hline \multirow[t]{2}{*}{ NHW } & 145 & $07-09-62$ & 4.97 & $s$ \\
\hline & & $09-05-62$ & 4.25 & $s$ \\
\hline \multirow[t]{2}{*}{ NHW } & 148 & $07-09-62$ & 10.62 & $s$ \\
\hline & & 09.01 .62 & 12.27 & $\mathbf{s}$ \\
\hline \multirow[t]{4}{*}{ NHW } & 149 & $07 \cdot 10 \cdot 62$ & 6.42 & $s$ \\
\hline & & $07-26-62$ & 7.51 & $s$ \\
\hline & & $08-13-62$ & 8.21 & $s$ \\
\hline & & $09-01-62$ & 8.77 & $\mathbf{s}$ \\
\hline \multirow[t]{2}{*}{ NHW } & 150 & $07-10-62$ & 23.88 & $s$ \\
\hline & & $09-05-62$ & 24.30 & $s$ \\
\hline \multirow[t]{4}{*}{ NHW } & 151 & $07-10-62$ & 6.63 & $s$ \\
\hline & & $09-01-62$ & 6.83 & $\mathbf{s}$ \\
\hline & & $09-25-62$ & 9.15 & $s$ \\
\hline & & $06-16-88$ & 5.0 & $T$ \\
\hline \multirow[t]{2}{*}{ NHW } & 152 & $07-10-62$ & 22.29 & $s$ \\
\hline & & $09-01-62$ & 23.23 & $\mathbf{s}$ \\
\hline \multirow[t]{4}{*}{ NHW } & 153 & $07-13-62$ & 18.58 & $s$ \\
\hline & & $08-19-62$ & 19.60 & $\mathbf{s}$ \\
\hline & & $06 \cdot 07-89$ & 16.08 & $\mathbf{s}$ \\
\hline & & $06-21-89$ & 16.46 & $s$ \\
\hline \multirow[t]{3}{*}{ NHW } & 154 & $07-13-62$ & 8.82 & s \\
\hline & & $09-01 \cdot 62$ & 11.63 & $\mathbf{S}$ \\
\hline & & $08-09 \cdot 62$ & 10.63 & $\mathbf{S}$ \\
\hline \multirow[t]{3}{*}{ NHW } & 156 & $08-30-62$ & 15.19 & $\mathbf{s}$ \\
\hline & & $04-21-88$ & 14.77 & $s$ \\
\hline & & $08-23-88$ & 15.89 & $S$ \\
\hline \multirow[t]{3}{*}{ NHW } & 157 & $07-14-62$ & 5.31 & $\mathbf{S}$ \\
\hline & & $09-06-62$ & 5.09 & s \\
\hline & & $04-21-88$ & 3.94 & $S$ \\
\hline \multirow[t]{4}{*}{ NHW } & 158 & $08-06-62$ & 72.64 & $s$ \\
\hline & & $09-03-62$ & 72.82 & $\mathbf{s}$ \\
\hline & & $05-23-63$ & 73.29 & $s$ \\
\hline & & $05-24-63$ & 73.38 & $s$ \\
\hline \multirow[t]{2}{*}{ NHW } & 159 & $07-17-62$ & 4.05 & $s$ \\
\hline & & $09-06-62$ & 4.22 & $\mathbf{s}$ \\
\hline \multirow[t]{2}{*}{ NHW } & 162 & $07-18-62$ & 5.22 & s \\
\hline & & $09-01-62$ & 4.45 & $s$ \\
\hline
\end{tabular}


Table 5.--Periodic water levels in selected wells--Continued

[Water levels are in feet below land surface; Measurement methods: $E$, estimated to nearest $f t$; $R$, reported to nearest $\mathrm{ft}$; $S$, steel tape, measured to nearest $0.01 \mathrm{ft}$; $T$, electric tape, measured to nearest $0.1 \mathrm{ft}$; $Z$, acoustic meter, measured to nearest $0.1 \mathrm{ft}]$

\begin{tabular}{lclc}
\hline & Date & & Method \\
& water & Water & water \\
Station & level & level & level \\
number & measured & (feet) & measured \\
\hline
\end{tabular}

\begin{tabular}{lccc}
\hline & Date & & Method \\
& water & Water & water \\
level & level & level \\
Station & measured & (feet) & measured \\
number & &
\end{tabular}

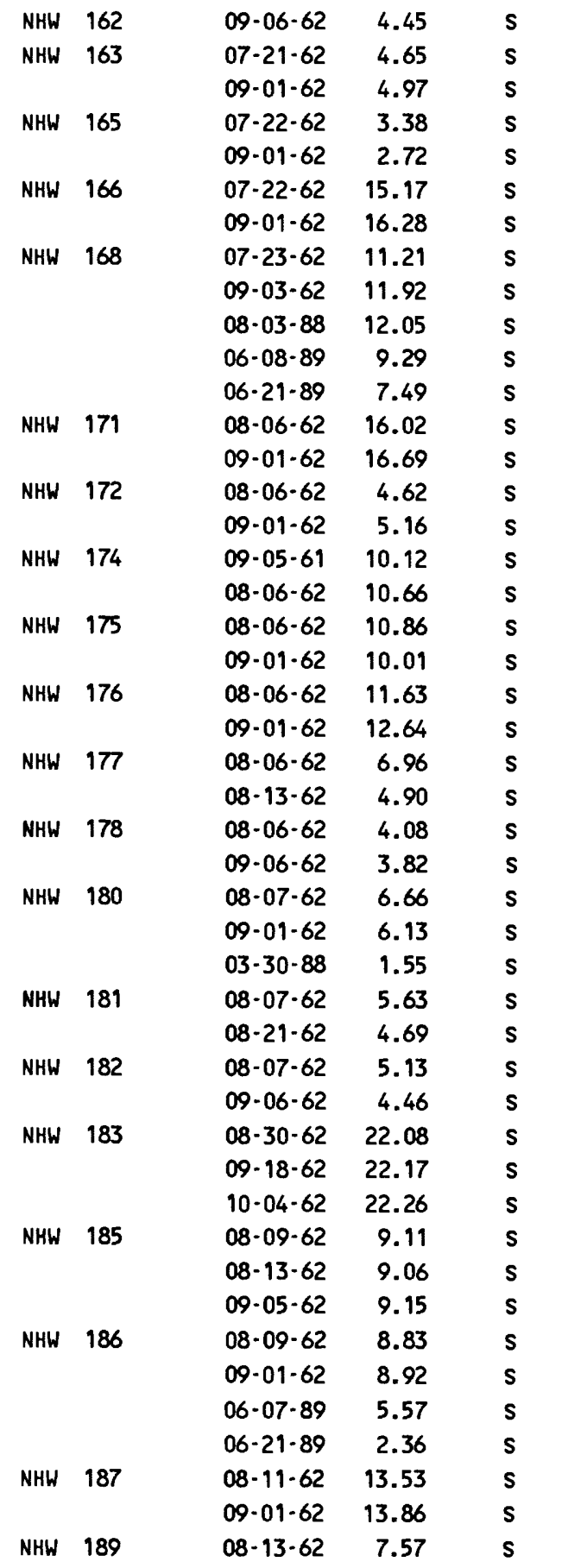

\begin{tabular}{|c|c|c|c|c|}
\hline NHW & 189 & $09-01-62$ & 8.33 & $\mathbf{s}$ \\
\hline \multirow[t]{3}{*}{ NHW } & 190 & $08-13-62$ & 6.13 & $\mathbf{s}$ \\
\hline & & $09-05-62$ & 6.03 & s \\
\hline & & $09-22-62$ & 6.66 & $\mathbf{s}$ \\
\hline \multirow[t]{2}{*}{ NHW } & 191 & $08-15 \cdot 62$ & 6.57 & $\mathbf{s}$ \\
\hline & & $09-01-62$ & 5.95 & s \\
\hline \multirow[t]{3}{*}{ NHW } & 193 & $06-00-60$ & 39.12 & $\mathbf{s}$ \\
\hline & & $08-16 \cdot 62$ & 38.98 & $\mathbf{s}$ \\
\hline & & $06-15-88$ & 38.15 & $\mathbf{s}$ \\
\hline \multirow[t]{3}{*}{ NHW } & 207 & $08-21-62$ & 5.70 & $s$ \\
\hline & & $09-01-62$ & 5.12 & $\mathbf{s}$ \\
\hline & & $06-22-88$ & 2.92 & s \\
\hline \multirow[t]{2}{*}{ NHW } & 208 & $08-22 \cdot 62$ & 19.45 & $s$ \\
\hline & & $09-02-62$ & 19.67 & $\mathbf{s}$ \\
\hline \multirow[t]{3}{*}{ NHW } & 209 & $08 \cdot 30 \cdot 62$ & 3.47 & $\mathbf{s}$ \\
\hline & & $05-24-63$ & 3.52 & s \\
\hline & & $05-04-88$ & 3.93 & $\mathbf{s}$ \\
\hline \multirow[t]{4}{*}{ NHW } & 215 & $08-31-62$ & 7.72 & $\mathbf{s}$ \\
\hline & & $09-08-62$ & 7.77 & $\mathbf{s}$ \\
\hline & & $09-17-62$ & 8.49 & $\mathbf{s}$ \\
\hline & & $10-31-90$ & 9.44 & $\mathbf{s}$ \\
\hline \multirow[t]{5}{*}{ NHW } & 216 & $08-31 \cdot 62$ & 14.11 & s \\
\hline & & $09-06-62$ & 14.19 & $\mathbf{s}$ \\
\hline & & $10-06-62$ & 15.29 & $\mathbf{s}$ \\
\hline & & $06-08 \cdot 89$ & 9.16 & $\mathbf{s}$ \\
\hline & & $06-21-89$ & 7.59 & $\mathbf{s}$ \\
\hline \multirow[t]{4}{*}{ NHW } & 217 & $08 \cdot 31-62$ & 35.15 & $\mathbf{s}$ \\
\hline & & $09.09-62$ & 35.14 & $\mathbf{s}$ \\
\hline & & $10 \cdot 10-62$ & 35.30 & $\mathbf{s}$ \\
\hline & & $06 \cdot 20-89$ & 8 & $\mathbf{R}$ \\
\hline \multirow[t]{2}{*}{ NHW } & 219 & $09 \cdot 03.62$ & 19.59 & s \\
\hline & & $08-05-88$ & 19.40 & $s$ \\
\hline \multirow[t]{3}{*}{ NHW } & 220 & 09.06 .62 & 8.94 & s \\
\hline & & $06 \cdot 15 \cdot 89$ & 2.84 & $\mathbf{s}$ \\
\hline & & $06-21 \cdot 89$ & 3.44 & $\mathbf{s}$ \\
\hline \multirow[t]{2}{*}{ NHW } & 222 & $09-07-62$ & 35.44 & $\mathbf{s}$ \\
\hline & & $10-31 \cdot 90$ & 4.58 & $\mathbf{s}$ \\
\hline \multirow[t]{2}{*}{ NHW } & 225 & $09 \cdot 16 \cdot 62$ & 37.79 & s \\
\hline & & $09-23-62$ & 37.87 & $\mathbf{s}$ \\
\hline \multirow[t]{7}{*}{ NHW } & 241 & $03-28-88$ & 12.37 & $\mathbf{s}$ \\
\hline & & $04-12-89$ & 12 & $\mathbf{R}$ \\
\hline & & $04-29-89$ & 12.19 & $\mathbf{s}$ \\
\hline & & $05-16-89$ & 12.39 & $\mathbf{s}$ \\
\hline & & $06-09-89$ & 11.2 & $T$ \\
\hline & & $06-21-89$ & 11 & $\mathbf{R}$ \\
\hline & & $08-10 \cdot 90$ & 12.99 & $\mathbf{s}$ \\
\hline
\end{tabular}


Table 5.--Periodic water levels in selected wells--Continued

[Water levels are in feet below land surface; Measurement methods: $E$, estimated to nearest ft; $R$, reported to nearest $\mathrm{ft}$; $S$, steel tape, measured to nearest $0.01 \mathrm{ft}$; $T$, electric tape, measured to nearest $0.1 \mathrm{ft} ; Z$, acoustic meter, measured to nearest $0.1 \mathrm{ft}]$

\begin{tabular}{|c|c|c|c|c|}
\hline \multicolumn{2}{|c|}{$\begin{array}{l}\text { Station } \\
\text { number }\end{array}$} & $\begin{array}{l}\text { Date } \\
\text { water } \\
\text { level } \\
\text { measured }\end{array}$ & $\begin{array}{l}\text { Water } \\
\text { level } \\
\text { (feet) }\end{array}$ & $\begin{array}{l}\text { Method } \\
\text { water } \\
\text { level } \\
\text { measured }\end{array}$ \\
\hline NHW & 241 & $06 \cdot 14 \cdot 91$ & 13.35 & s \\
\hline \multirow[t]{4}{*}{ NHW } & 250 & $10 \cdot 23 \cdot 89$ & 35.79 & $s$ \\
\hline & & $08 \cdot 30 \cdot 90$ & 33.70 & $s$ \\
\hline & & $09-28-90$ & 34.32 & $s$ \\
\hline & & $06-14-91$ & 36.13 & $s$ \\
\hline \multirow[t]{4}{*}{ NHW } & 251 & $10 \cdot 23 \cdot 89$ & 8.13 & $s$ \\
\hline & & $08-30-90$ & 10.53 & $s$ \\
\hline & & $09-28-90$ & 10.64 & $\mathbf{s}$ \\
\hline & & $06-14-91$ & 8.42 & $\mathbf{s}$ \\
\hline \multirow[t]{4}{*}{ NHW } & 252 & $10-24-89$ & 42.06 & $s$ \\
\hline & & $08 \cdot 30 \cdot 90$ & 42.95 & $\mathbf{s}$ \\
\hline & & $11-06-90$ & 42.34 & $\mathbf{s}$ \\
\hline & & $06-14-91$ & 42.2 & $T$ \\
\hline \multirow[t]{3}{*}{ NHW } & 253 & $10-24-89$ & 4.98 & $\mathbf{s}$ \\
\hline & & $08 \cdot 30-90$ & 6.70 & $\mathbf{s}$ \\
\hline & & $11-06-90$ & 9.89 & $s$ \\
\hline NHW & 253 & $06 \cdot 14 \cdot 91$ & 4.75 & $\mathbf{s}$ \\
\hline \multirow[t]{2}{*}{ NHW } & 254 & $10-24-89$ & 4.08 & $\mathbf{s}$ \\
\hline & & $11 \cdot 01-90$ & 7.68 & $s$ \\
\hline \multirow[t]{3}{*}{ NHW } & 255 & $10-23-89$ & 9.31 & $s$ \\
\hline & & $08 \cdot 28 \cdot 90$ & 12.43 & s \\
\hline & & $06-13-91$ & 8.79 & $\mathbf{s}$ \\
\hline \multirow[t]{4}{*}{ NHW } & 256 & $10-25-89$ & 81.10 & $\mathbf{s}$ \\
\hline & & $08-27-90$ & 78.0 & $T$ \\
\hline & & $09-21-90$ & 78.2 & $T$ \\
\hline & & $06-13-91$ & 80.8 & $T$ \\
\hline \multirow[t]{4}{*}{ NHW } & 257 & $10-25-89$ & 25.04 & $s$ \\
\hline & & $08 \cdot 27 \cdot 90$ & 24.30 & $s$ \\
\hline & & $09-21-90$ & 24.86 & $\mathbf{s}$ \\
\hline & & $06-13-91$ & 23.63 & $s$ \\
\hline \multirow[t]{4}{*}{ NHW } & 258 & $10-25-89$ & 11.52 & $s$ \\
\hline & & $08-27-90$ & 11.99 & s \\
\hline & & $09-21 \cdot 90$ & 12.52 & $s$ \\
\hline & & $06-13 \cdot 91$ & 11.25 & $\mathbf{s}$ \\
\hline \multirow[t]{3}{*}{ NHW } & 259 & $10 \cdot 23-89$ & 9.79 & $s$ \\
\hline & & $08-30-90$ & 10.09 & s \\
\hline & & $11-06-90$ & 9.96 & s \\
\hline \multirow[t]{3}{*}{ NHW } & 260 & $10-23-89$ & 9.34 & $s$ \\
\hline & & $08-30-90$ & 10.00 & $s$ \\
\hline & & $11-06-90$ & 9.68 & s \\
\hline \multirow[t]{3}{*}{ NHW } & 261 & $10 \cdot 23 \cdot 89$ & 8.09 & $\mathbf{s}$ \\
\hline & & $08-30-90$ & 8.39 & $\mathbf{s}$ \\
\hline & & $11-06 \cdot 90$ & 8.71 & $s$ \\
\hline \multirow[t]{3}{*}{ NHW } & 263 & $10-25-89$ & 44.79 & s \\
\hline & & $11-01.91$ & 44.21 & s \\
\hline & & $06-14 \cdot 91$ & 45.9 & $T$ \\
\hline
\end{tabular}

\begin{tabular}{|c|c|c|c|c|}
\hline \multicolumn{2}{|c|}{$\begin{array}{l}\text { Station } \\
\text { number }\end{array}$} & $\begin{array}{l}\text { Date } \\
\text { water } \\
\text { level } \\
\text { measured }\end{array}$ & $\begin{array}{l}\text { Water } \\
\text { level } \\
\text { (feet) }\end{array}$ & \multirow{2}{*}{$\begin{array}{l}\text { Method } \\
\text { water } \\
\text { level } \\
\text { measured } \\
\text { S }\end{array}$} \\
\hline NHW & 264 & $10-25-89$ & 9.25 & \\
\hline & & $08-30-90$ & 9.86 & $\mathbf{s}$ \\
\hline & & $11 \cdot 06-90$ & 10.13 & $s$ \\
\hline \multirow[t]{3}{*}{ NHW } & 322 & $03-09-88$ & 89.80 & $\mathbf{s}$ \\
\hline & & $06-09-89$ & 89.9 & $T$ \\
\hline & & $06-21-89$ & 90.1 & $T$ \\
\hline \multirow[t]{2}{*}{ NHW } & 325 & $05-19-82$ & 115 & $\mathbf{R}$ \\
\hline & & $07-11-89$ & 115.6 & $z$ \\
\hline \multirow[t]{2}{*}{ NHW } & 328 & $08.06 \cdot 79$ & 124 & $\mathbf{R}$ \\
\hline & & $06-29-89$ & 128.0 & $z$ \\
\hline \multirow[t]{2}{*}{ NHW } & 329 & $08 \cdot 12 \cdot \pi$ & 102 & $\mathbf{R}$ \\
\hline & & $06-29-89$ & 96.3 & $z$ \\
\hline \multirow[t]{3}{*}{ NHW } & 333 & $03-10-88$ & 160.5 & $T$ \\
\hline & & $06-09-89$ & 160.6 & $T$ \\
\hline & & $06 \cdot 21 \cdot 89$ & 160.36 & s \\
\hline \multirow[t]{4}{*}{ NHW } & 335 & $03-11-88$ & 140.4 & $T$ \\
\hline & & $06 \cdot 09-89$ & 140.0 & $T$ \\
\hline & & $06 \cdot 21 \cdot 89$ & 139.69 & $s$ \\
\hline & & $06-14-91$ & 144.4 & $T$ \\
\hline \multirow[t]{3}{*}{ NHW } & 337 & $09-18-85$ & 110 & $\mathbf{R}$ \\
\hline & & $06-14-89$ & 106.3 & 2 \\
\hline & & $06-21-89$ & 106.4 & $z$ \\
\hline \multirow[t]{2}{*}{ NHW } & 341 & $08 \cdot 04 \cdot 76$ & 118 & $\mathbf{R}$ \\
\hline & & $06 \cdot 20-89$ & 118 & $\mathbf{R}$ \\
\hline \multirow[t]{2}{*}{ NHW } & 369 & $07 \cdot 19-66$ & 30 & $\mathbf{R}$ \\
\hline & & $06 \cdot 20-89$ & 45.9 & $z$ \\
\hline \multirow[t]{2}{*}{ NHW } & 374 & $09 \cdot 30-65$ & 42 & $\mathbf{R}$ \\
\hline & & $07-07-89$ & 39.0 & $z$ \\
\hline \multirow[t]{2}{*}{ NHW } & 383 & $09 \cdot 29 \cdot 77$ & 38 & $\mathbf{R}$ \\
\hline & & $07-07-89$ & 34.8 & $T$ \\
\hline \multirow[t]{2}{*}{ NHW } & 391 & $05 \cdot 05 \cdot 76$ & 16 & $\mathbf{R}$ \\
\hline & & $06-14-89$ & 20.2 & $z$ \\
\hline NHW & 391 & $06-21 \cdot 89$ & 18.1 & $z$ \\
\hline \multirow[t]{2}{*}{ NHW } & 401 & $06-25 \cdot 81$ & 23 & $\mathbf{R}$ \\
\hline & & $07-11-89$ & 27 & $\mathbf{R}$ \\
\hline \multirow[t]{2}{*}{ NHW } & 402 & $05-19-76$ & 60 & $\mathbf{R}$ \\
\hline & & $07-11-89$ & 71 & $\mathbf{R}$ \\
\hline \multirow[t]{2}{*}{ NHW } & 412 & $08-07-64$ & 28 & $R$ \\
\hline & & $06-29-89$ & 31.2 & $z$ \\
\hline \multirow[t]{5}{*}{ NHW } & 417 & $03-29-88$ & 124.45 & $s$ \\
\hline & & $03-29 \cdot 88$ & 124.45 & $s$ \\
\hline & & $04-29-89$ & 123.60 & s \\
\hline & & $05-16-89$ & 123.90 & $s$ \\
\hline & & $06-14 \cdot 89$ & 123.6 & $T$ \\
\hline NHW & 424 & $06-21-89$ & 33.44 & $s$ \\
\hline
\end{tabular}


Table 5.--Periodic water levels in selected wells.-Continued

[Water levels are in feet below land surface; Measurement methods: $E$, estimated to nearest $f t$; $R$, reported to nearest $\mathrm{ft}$; $S$, steel tape, measured to nearest $0.01 \mathrm{ft}$; $T$, electric tape, measured to nearest $0.1 \mathrm{ft}$; $Z$, acoustic meter, measured to nearest $0.1 \mathrm{ft}]$

\begin{tabular}{lccc}
\hline & Date & & Method \\
& water & Water & water \\
Station & level & level & level \\
number & measured & (feet) & measured \\
\hline
\end{tabular}

\begin{tabular}{|c|c|c|c|c|}
\hline \multirow[t]{3}{*}{ NHW } & 424 & $06-21-89$ & 33 & $\mathbf{R}$ \\
\hline & & $08-10-90$ & 24.21 & $\mathbf{S}$ \\
\hline & & $06-14-91$ & 31.6 & $T$ \\
\hline \multirow[t]{4}{*}{ NHW } & 429 & $03-09-88$ & 2.6 & $T$ \\
\hline & & $04-20-89$ & 2.86 & $\mathbf{S}$ \\
\hline & & $06-09 \cdot 89$ & 2.74 & $S$ \\
\hline & & $06-21-89$ & 2.70 & S \\
\hline \multirow[t]{2}{*}{ NHW } & 468 & $06-19-89$ & 39.46 & $S$ \\
\hline & & $08-16-89$ & 39.9 & $z$ \\
\hline \multirow[t]{3}{*}{ NHW } & 523 & $09-04-79$ & 38 & $\mathbf{R}$ \\
\hline & & $06-07 \cdot 89$ & 43.0 & $z$ \\
\hline & & $06-21 \cdot 89$ & 43.8 & Z \\
\hline \multirow[t]{3}{*}{ NHW } & 529 & $08-05 \cdot 80$ & 121 & $\mathbf{R}$ \\
\hline & & $06-15-89$ & 120.62 & s \\
\hline & & $06-21-89$ & 120.7 & $z$ \\
\hline \multirow[t]{2}{*}{ NHW } & 534 & $05-23 \cdot 75$ & 94 & $\mathbf{R}$ \\
\hline & & $07-11-89$ & 94 & $\mathbf{R}$ \\
\hline \multirow[t]{3}{*}{ NHW } & 535 & $08-07-68$ & 123 & $\mathbf{R}$ \\
\hline & & $06-27-89$ & 125 & $\mathbf{R}$ \\
\hline & & $06-13-91$ & 122.6 & $T$ \\
\hline \multirow[t]{3}{*}{ NHW } & 538 & $07-27-72$ & 99 & $\mathbf{R}$ \\
\hline & & $06-08-89$ & 94.9 & $z$ \\
\hline & & $06-21-89$ & 94.9 & Z \\
\hline \multirow[t]{2}{*}{ NHW } & 542 & $10-15 \cdot 71$ & 152 & $\mathbf{R}$ \\
\hline & & $06-27-89$ & 149.2 & $T$ \\
\hline \multirow[t]{3}{*}{ NHW } & 547 & $09 \cdot 17 \cdot 79$ & 168 & $\mathbf{R}$ \\
\hline & & $06-26-89$ & 154.5 & $z$ \\
\hline & & $07-11-89$ & 154 & $\mathbf{R}$ \\
\hline \multirow[t]{2}{*}{ NHW } & 548 & $07-27-77$ & 120 & $\mathbf{R}$ \\
\hline & & $06-29-89$ & 119.3 & $z$ \\
\hline \multirow[t]{2}{*}{ NHW } & 552 & $10-11 \cdot 66$ & 130 & $\mathbf{R}$ \\
\hline & & $06-13-91$ & 154.0 & $T$ \\
\hline \multirow[t]{2}{*}{ NHW } & 555 & $05 \cdot 20 \cdot 70$ & 117 & $\mathbf{R}$ \\
\hline & & $06 \cdot 29-89$ & 113.8 & z \\
\hline \multirow[t]{2}{*}{ NHW } & 556 & $09-09-83$ & 170 & $\mathbf{R}$ \\
\hline & & $06-29-89$ & 165.8 & $T$ \\
\hline \multirow[t]{3}{*}{ NHW } & 557 & $06 \cdot 30-70$ & 170 & $\mathbf{R}$ \\
\hline & & $06-27-89$ & 168.5 & $z$ \\
\hline & & $06-13-91$ & 174.0 & $T$ \\
\hline \multirow[t]{3}{*}{ NHW } & 564 & $09-14-82$ & 58 & $\mathbf{R}$ \\
\hline & & $06-07-89$ & 55.6 & 2 \\
\hline & & $06-21-89$ & 55.5 & $z$ \\
\hline \multirow[t]{2}{*}{ NHW } & 565 & $05-15-69$ & 78 & $\mathbf{R}$ \\
\hline & & $06-07-89$ & 59.5 & $\mathbf{Z}$ \\
\hline \multirow[t]{2}{*}{ NHW } & 566 & $09-15-82$ & 60 & $\mathbf{R}$ \\
\hline & & $06-07-89$ & 49.6 & 2 \\
\hline
\end{tabular}

\begin{tabular}{lclc}
\hline & Date & & Method \\
& water & Water & water \\
Station & level & level & level \\
number & measured & (feet) & measured
\end{tabular}

\begin{tabular}{|c|c|c|c|c|}
\hline NHW & 566 & $06-20-89$ & 60.6 & $z$ \\
\hline \multirow[t]{2}{*}{ NHW } & 567 & $07-13-82$ & 100 & $\mathbf{R}$ \\
\hline & & $07-11-89$ & 97 & $\mathbf{R}$ \\
\hline \multirow[t]{4}{*}{ NHW } & 570 & $10-08-71$ & 136 & $R$ \\
\hline & & $06-07-89$ & 134.4 & $z$ \\
\hline & & $06-21-89$ & 135.2 & z \\
\hline & & $06-14-91$ & 138.8 & $\mathbf{T}$ \\
\hline \multirow[t]{3}{*}{ NHW } & 577 & $08-21-68$ & 56 & $\mathbf{R}$ \\
\hline & & $06 \cdot 27 \cdot 89$ & 50 & $\mathbf{R}$ \\
\hline & & $10-24-90$ & 47.53 & s \\
\hline \multirow[t]{3}{*}{ NHW } & 627 & $12 \cdot 15 \cdot 88$ & 16.1 & z \\
\hline & & $06-20-89$ & 6.57 & $\mathbf{s}$ \\
\hline & & $06-14-91$ & 10.79 & $\mathbf{s}$ \\
\hline \multirow[t]{8}{*}{ NHW } & 628 & $12-15-88$ & 18.7 & Z \\
\hline & & $01 \cdot 18 \cdot 89$ & 16.88 & $\mathbf{S}$ \\
\hline & & $03-28 \cdot 89$ & 16.55 & $\mathbf{s}$ \\
\hline & & $04-21-89$ & 13.85 & $\mathbf{S}$ \\
\hline & & $05-09-89$ & 13.45 & $\mathbf{S}$ \\
\hline & & $06-09-89$ & 12.6 & z \\
\hline & & $06-21-89$ & 12.63 & $\mathbf{S}$ \\
\hline & & $06-13-91$ & 14.71 & $\mathbf{S}$ \\
\hline \multirow[t]{9}{*}{ NHW } & 629 & $12-15-88$ & 16.8 & $\mathbf{S}$ \\
\hline & & $01-17-89$ & 8 & $\mathbf{R}$ \\
\hline & & $03-28-89$ & 3.50 & $\mathbf{S}$ \\
\hline & & $04-20-89$ & 3.27 & S \\
\hline & & $05-09 \cdot 89$ & 4.02 & $\mathrm{~S}$ \\
\hline & & $06-09 \cdot 89$ & 11.5 & Z \\
\hline & & $06-21-89$ & 3.42 & $\mathbf{s}$ \\
\hline & & $08 \cdot 28-90$ & 9.66 & $\mathbf{S}$ \\
\hline & & $06-13-91$ & 5.98 & $\mathbf{s}$ \\
\hline \multirow[t]{2}{*}{ NHW } & 630 & $03-28-89$ & 35.28 & $\mathbf{S}$ \\
\hline & & $06-14-91$ & 33.60 & S \\
\hline \multirow[t]{10}{*}{ NHW } & 632 & $12-15-88$ & 18.2 & z \\
\hline & & $01-18-89$ & 14.69 & $\mathbf{s}$ \\
\hline & & $03-28-89$ & 14.01 & $\mathbf{s}$ \\
\hline & & $04-20-89$ & 11.54 & $\mathbf{S}$ \\
\hline & & $05-09-89$ & 12.08 & $\mathbf{s}$ \\
\hline & & $06-09-89$ & 13.4 & Z \\
\hline & & $06-21 \cdot 89$ & 11.84 & $\mathbf{s}$ \\
\hline & & $08-27-90$ & 15.70 & s \\
\hline & & $09-28-90$ & 16.74 & $\mathbf{S}$ \\
\hline & & $06-13-91$ & 14.17 & $\mathbf{s}$ \\
\hline \multirow[t]{3}{*}{ NHW } & 636 & $06-08-89$ & 4.89 & s \\
\hline & & $06-08-89$ & 4.89 & S \\
\hline & & $06-21-89$ & 5.03 & $\mathbf{S}$ \\
\hline NHW & 660 & $06-26-84$ & 15 & $\mathbf{R}$ \\
\hline
\end{tabular}


Table 5.--Periodic water levels in selected wells - Continued

[Water levels are in feet below land surface; Measurement methods: $E$, estimated to nearest $f t$; $R$, reported to nearest $\mathrm{ft}$; $S$, steel tape, measured to nearest $0.01 \mathrm{ft}$; $\mathrm{T}$, electric tape, measured to nearest $0.1 \mathrm{ft}$; $Z$, acoustic meter, measured to nearest $0.1 \mathrm{ft}]$

\begin{tabular}{|c|c|c|c|c|}
\hline \multicolumn{2}{|c|}{$\begin{array}{l}\text { Station } \\
\text { number }\end{array}$} & \multirow{2}{*}{$\begin{array}{l}\text { Date } \\
\text { water } \\
\text { level } \\
\text { measured }\end{array}$} & \multirow{2}{*}{$\begin{array}{l}\begin{array}{l}\text { Water } \\
\text { level } \\
\text { (feet) }\end{array} \\
14.8\end{array}$} & \multirow{2}{*}{$\begin{array}{c}\text { Methoo } \\
\text { water } \\
\text { level } \\
\text { measure } \\
\mathrm{z}\end{array}$} \\
\hline NHW & 660 & & & \\
\hline \multirow[t]{2}{*}{ NHW } & 664 & $06-05-86$ & 3 & $\mathbf{R}$ \\
\hline & & $06-27-89$ & 2.00 & $\mathbf{s}$ \\
\hline \multirow[t]{2}{*}{ NHW } & 682 & $05-08-80$ & 138 & $\mathbf{R}$ \\
\hline & & $07-11-89$ & 138 & $\mathbf{R}$ \\
\hline \multirow[t]{3}{*}{ NHW } & 686 & $09 \cdot 16-69$ & 115 & $\mathbf{R}$ \\
\hline & & $06-13-89$ & 112.9 & z \\
\hline & & $06-21-89$ & 114.4 & z \\
\hline \multirow[t]{2}{*}{ NHW } & 687 & $05-07-82$ & 17 & $\mathbf{R}$ \\
\hline & & $06-27-89$ & 15 & $\mathbf{R}$ \\
\hline \multirow[t]{2}{*}{ NHW } & 695 & $07-01-71$ & 22 & $\mathbf{R}$ \\
\hline & & $07 \cdot 11-89$ & 18.6 & $T$ \\
\hline \multirow[t]{2}{*}{ NHW } & 722 & $08 \cdot 11-83$ & 75 & $\mathbf{R}$ \\
\hline & & $07-07-89$ & 56.9 & $T$ \\
\hline \multirow[t]{2}{*}{ NHW } & 750 & $06-07-89$ & 9.86 & s \\
\hline & & $06-21-89$ & 9.06 & $\mathbf{s}$ \\
\hline \multirow[t]{2}{*}{ NHW } & 752 & $07-09-85$ & 145 & $\mathbf{R}$ \\
\hline & & $06-20-89$ & 169 & $R$ \\
\hline \multirow[t]{3}{*}{ NHW } & 755 & $06-16-82$ & 56 & $\mathbf{R}$ \\
\hline & & $06-07-89$ & 51.4 & $z$ \\
\hline & & $06-21-89$ & 48.4 & 2 \\
\hline \multirow[t]{4}{*}{ NHW } & 756 & $05-17 \cdot 73$ & 8 & $\mathbf{R}$ \\
\hline & & $06-14-89$ & 1.70 & $\mathbf{s}$ \\
\hline & & $06-21-89$ & 1.80 & $\mathbf{s}$ \\
\hline & & $06-14-91$ & 4.20 & $\mathbf{s}$ \\
\hline \multirow[t]{2}{*}{ NHW } & 758 & $06-15-89$ & 0.81 & $\mathbf{s}$ \\
\hline & & $06-21-89$ & 1.67 & $\mathbf{s}$ \\
\hline \multirow[t]{2}{*}{ NHW } & 780 & $06 \cdot 09 \cdot 67$ & 38 & $\mathbf{R}$ \\
\hline & & $08-29-90$ & 37.80 & $\mathbf{s}$ \\
\hline
\end{tabular}

'Although E, estimated, and R, reported, are not measurement methods, these codes are used in the USGS Ground-Water site Inventory database. 



픔

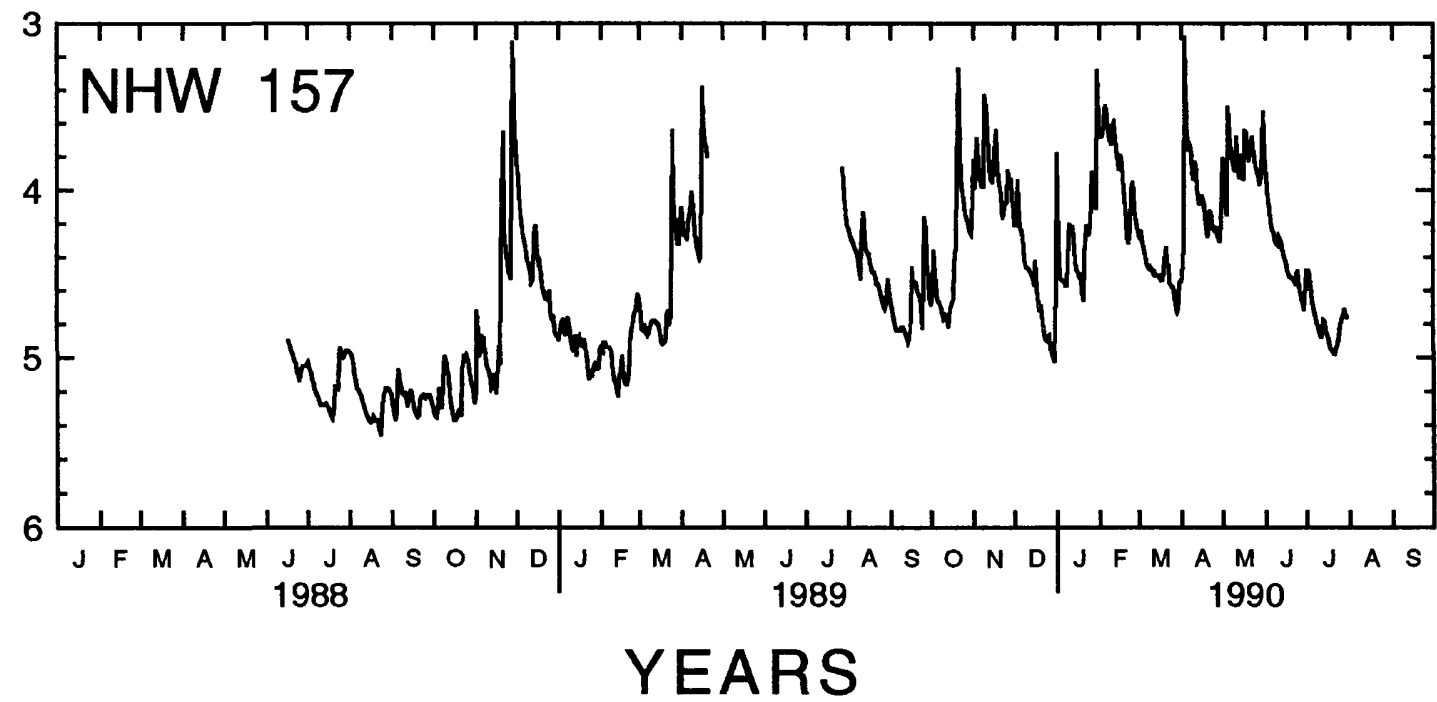

Figure 1.--Ground-water levels at selected wells (blank spaces represent missing data). 

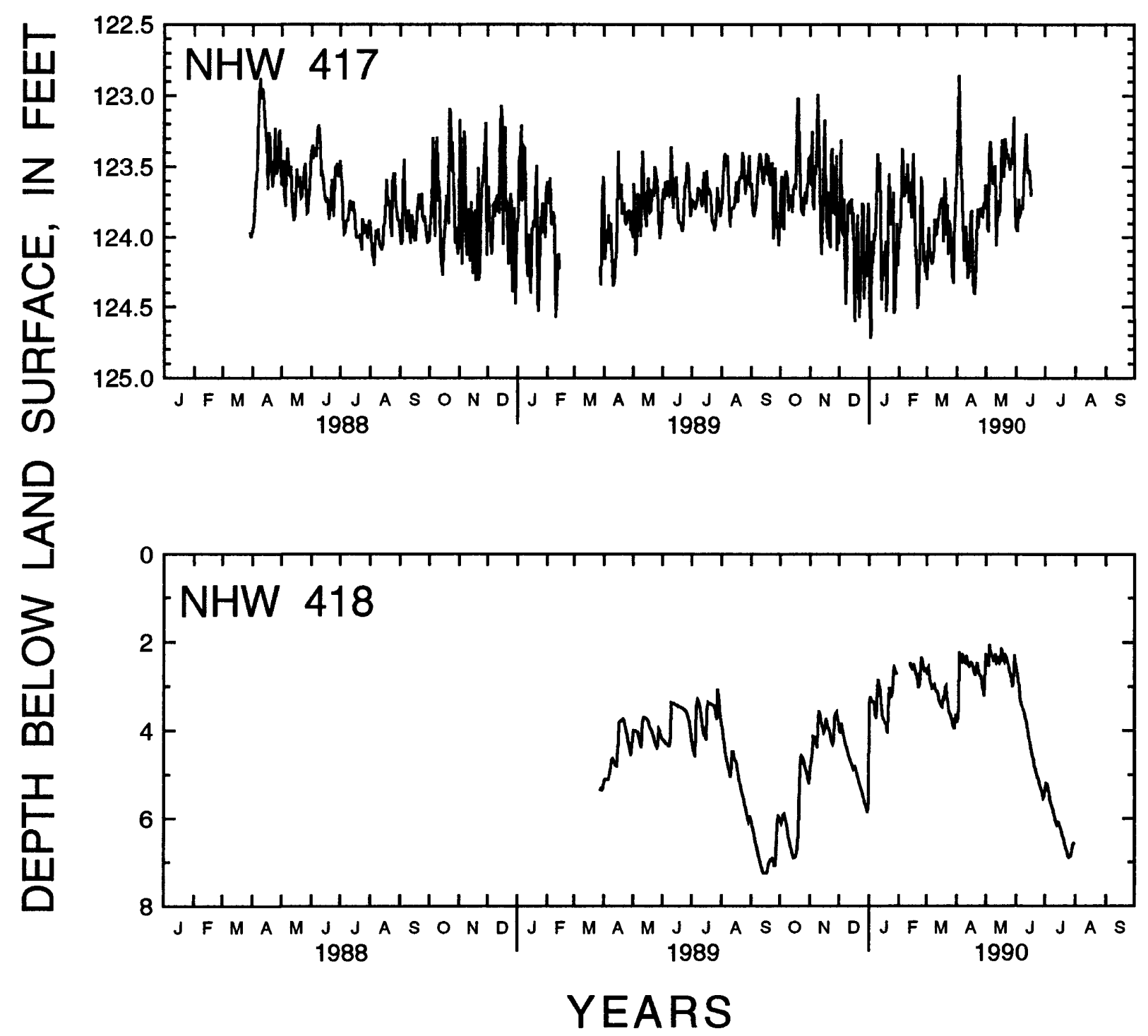

Figure 1.--Ground-water levels at selected wells--Continued. 




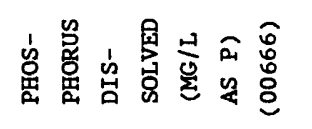

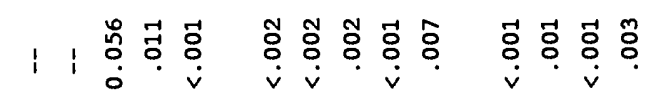

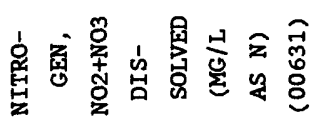

คैด

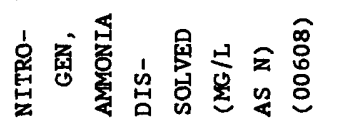

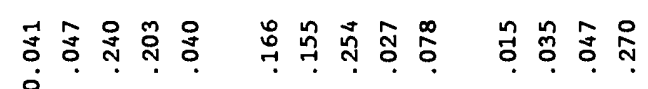

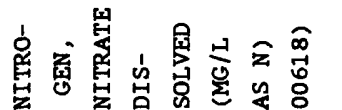

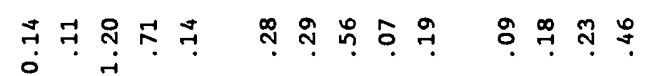

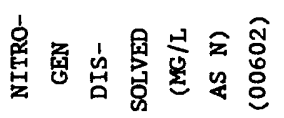

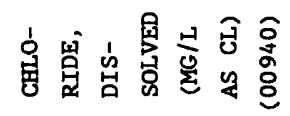

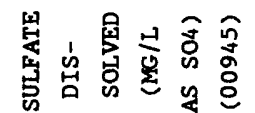

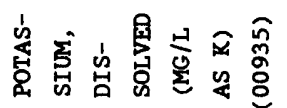

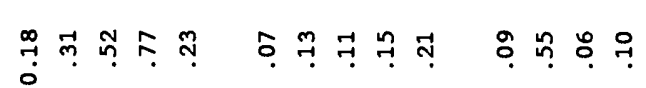

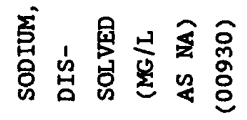

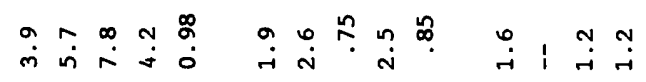

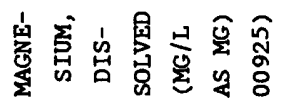



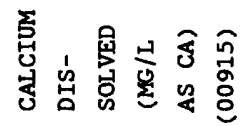

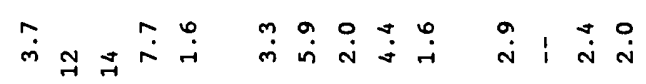

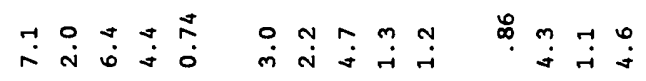

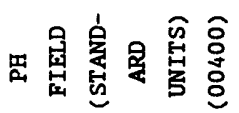

离



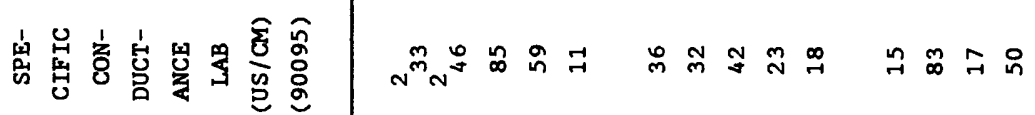

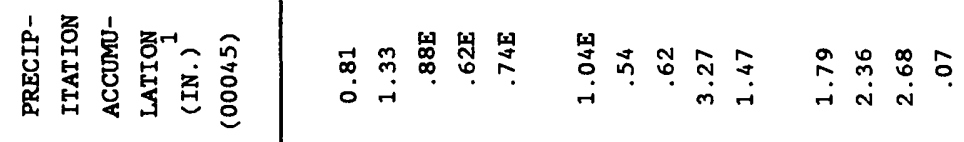

思

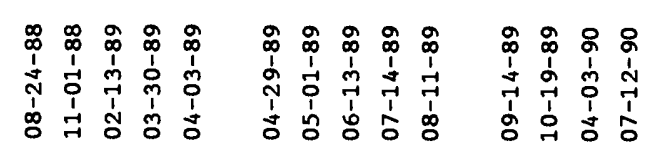




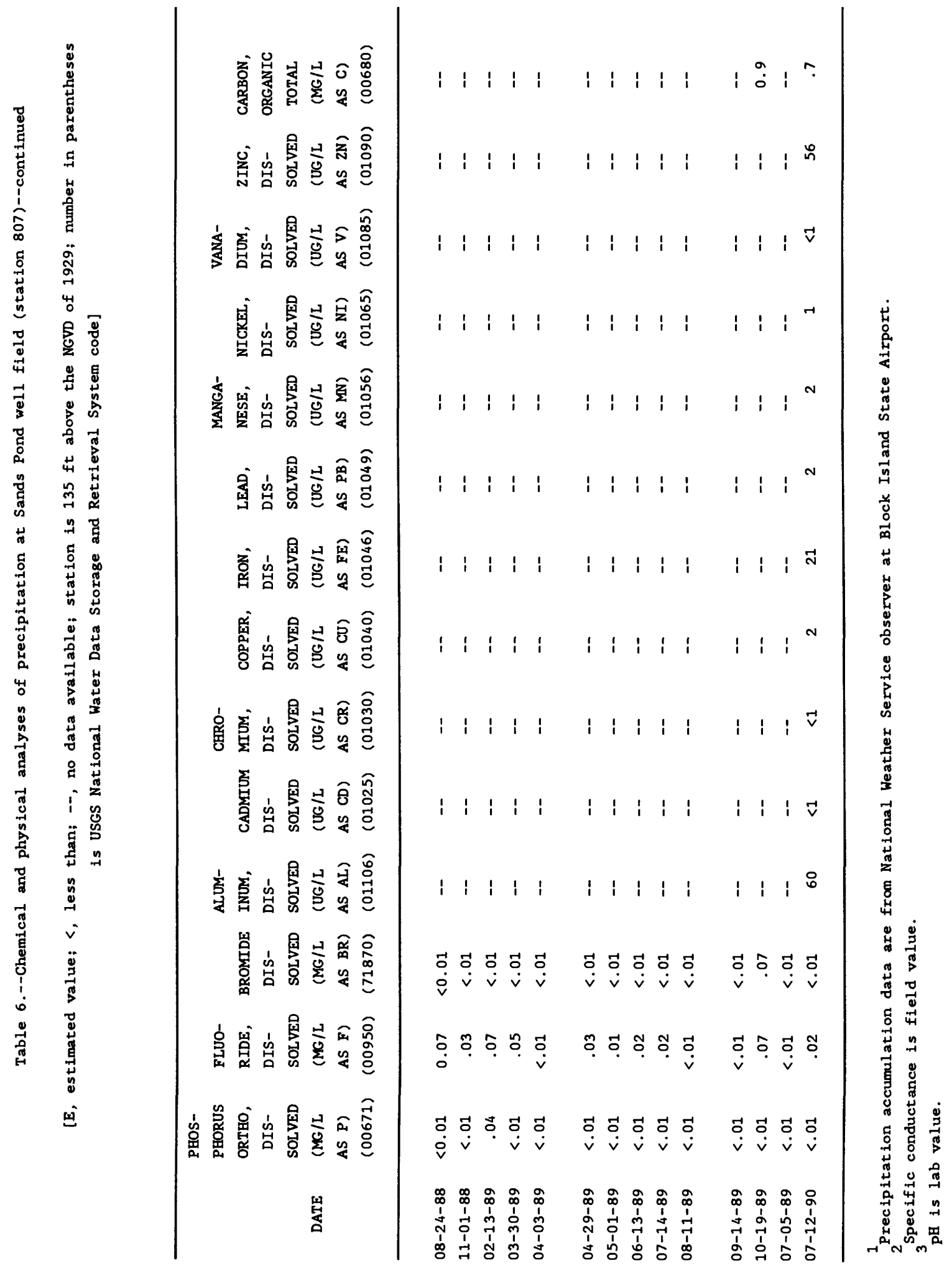




\begin{tabular}{|c|c|c|c|c|c|c|}
\hline 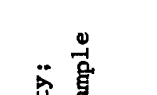 &  & 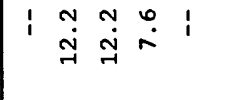 & $\dot{\sim} \infty$ & 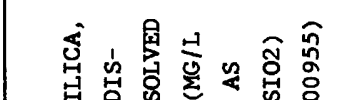 & $\mid \begin{array}{lllll}1 & 1 & 1 & 0 & 0 \\
1 & 1\end{array}$ & 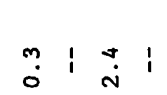 \\
\hline 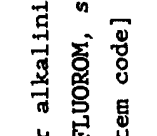 & 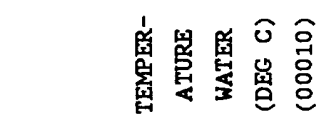 & $1: \hat{0}$ & 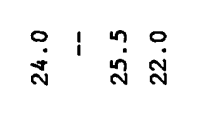 & 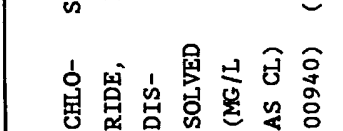 & 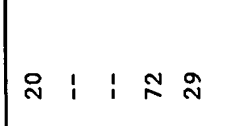 & 웍 요 \\
\hline 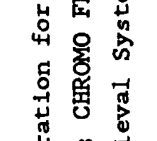 & 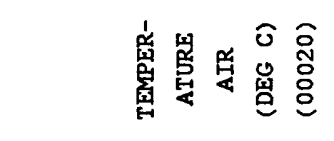 & 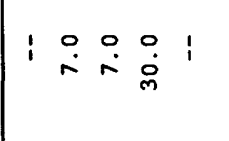 & 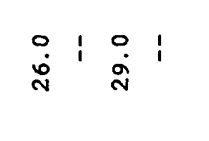 & 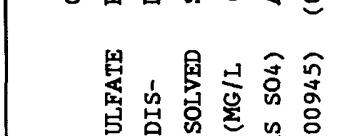 & $1: 11=1$ & m: 181 \\
\hline 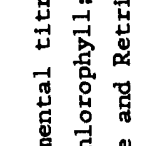 & 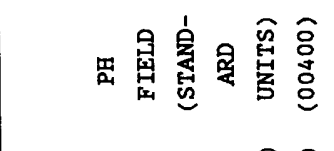 &  & 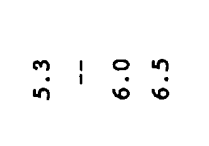 &  & $1: 1: 1 \quad 1 \quad F$ & $N: \sim$ \\
\hline 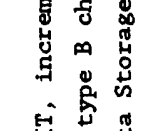 & 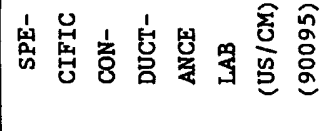 & 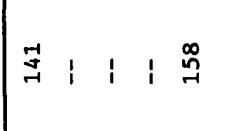 &  & 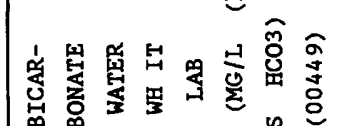 & $: 1: 1: F 1$ & $N:-1$ \\
\hline 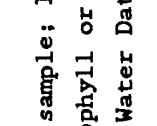 & 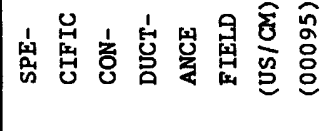 & 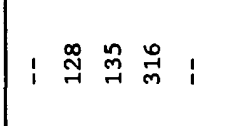 & 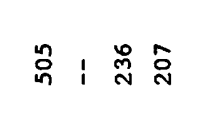 & 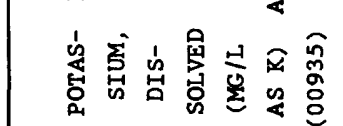 & 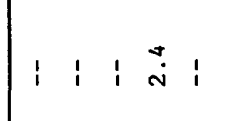 & 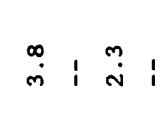 \\
\hline 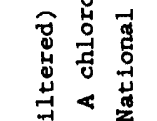 &  & 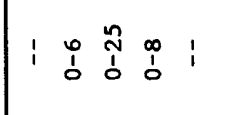 & 号: & 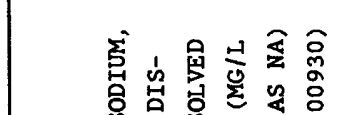 & 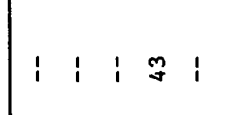 & 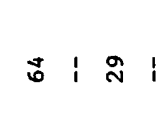 \\
\hline 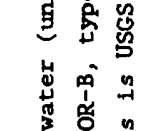 & 絭的䍚 & 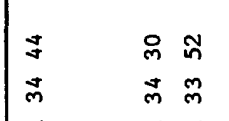 & 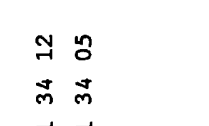 & 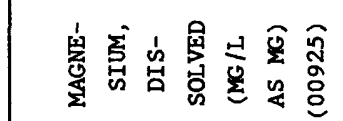 & $\mid \begin{array}{llllll}1 & 1 & 1 & 0 & 1 \\
& 0 & 1\end{array}$ & 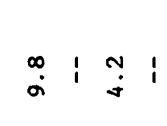 \\
\hline $\begin{array}{l}0 \\
0 \\
0\end{array}$ & & 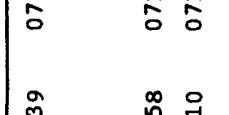 & $\begin{array}{l}5 \% \\
\approx \\
\approx\end{array}$ &  & $\mid \begin{array}{lllll}1 & 1 & 1 & \stackrel{n}{n} & 1\end{array}$ & $\begin{array}{l:ll}\infty & \infty \\
\dot{0} & \mid & \infty \\
\infty & 1\end{array}$ \\
\hline 畄兽 & & $\begin{array}{ll}0 & 72 \\
7 & 75 \\
7 & 7\end{array}$ & $\begin{array}{l}m 8 \\
\exists \exists\end{array}$ & 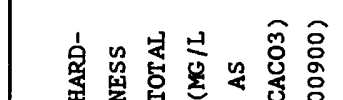 & $\vec{N}: \vec{A}:{ }^{\circ} \stackrel{\circ}{\circ}$ & in $\underset{\sim}{\infty} \hat{\alpha}$ : \\
\hline 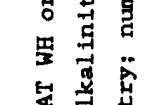 & 音 & 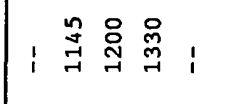 & 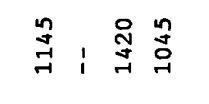 & 罢 & 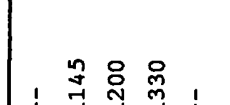 & 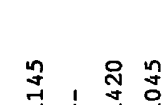 \\
\hline 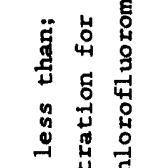 & 畧 & 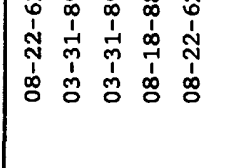 & 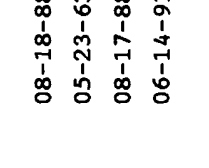 &  & 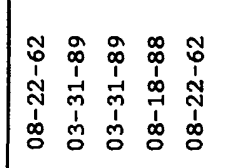 & 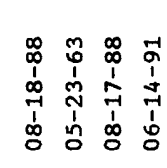 \\
\hline 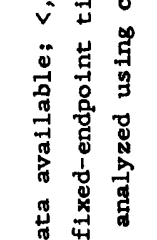 & & 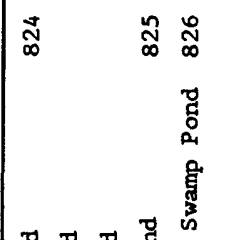 & $\stackrel{\substack{\infty \\
\infty}}{\infty}$ & 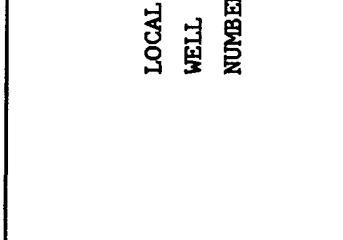 & 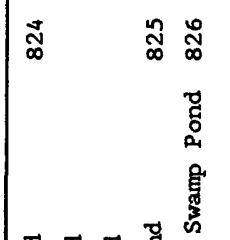 & $\widehat{్} \underset{\infty}{\infty}$ \\
\hline 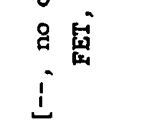 & 总㟟 & 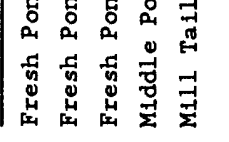 & 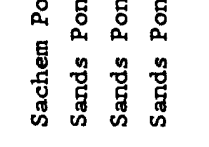 & 总 & 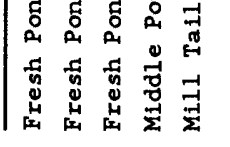 &  \\
\hline
\end{tabular}




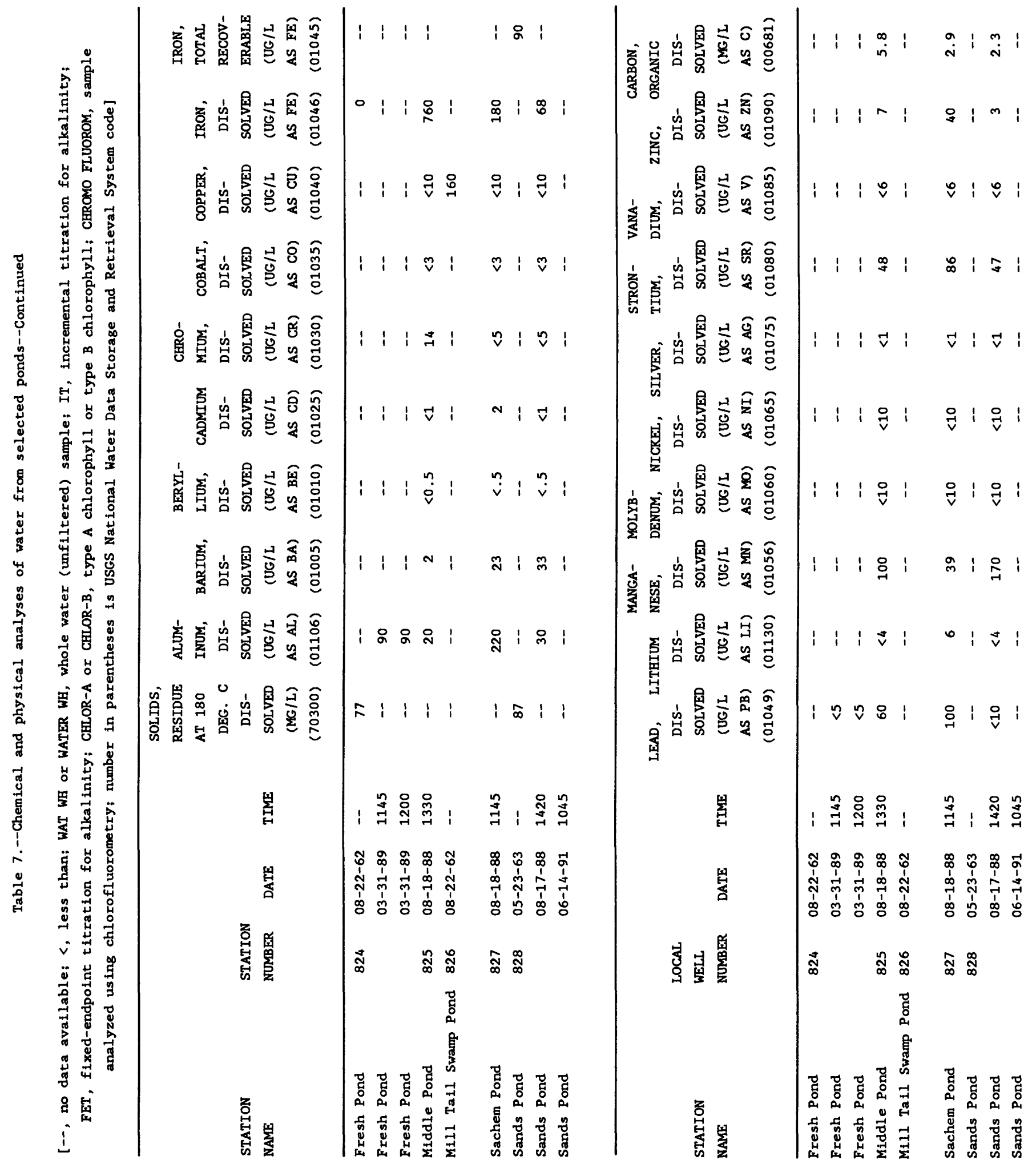







Table 8.--Chemical and physical analyses of

$[\cdots$, no data available; <, less than; WAT WH or

IT, incremental titration for alkalinity; FET,

number in parentheses is USGS National Water

\begin{tabular}{|c|c|c|c|c|c|c|c|c|c|c|}
\hline & & & & & $\begin{array}{l}\text { ELEV. } \\
\text { OF LAND }\end{array}$ & $\begin{array}{l}\text { SPE- } \\
\text { CIFIC }\end{array}$ & $\begin{array}{l}\text { SOLIDS, } \\
\text { RESIDUE }\end{array}$ & & & \\
\hline & & & & DEPTH & SURFACE & CON- & AT 180 & PH & & \\
\hline STATION & & $\begin{array}{l}\text { LAT - } \\
\text { I - }\end{array}$ & $\begin{array}{l}\text { LONG - } \\
\text { I- }\end{array}$ & $\begin{array}{c}\text { OF } \\
\text { WELL, }\end{array}$ & $\begin{array}{c}\text { DATUM } \\
\text { (FT. }\end{array}$ & $\begin{array}{l}\text { DUCT - } \\
\text { ANCE }\end{array}$ & $\begin{array}{l}\text { DEG. C } \\
\text { DIS- }\end{array}$ & $\begin{array}{l}\text { FIELD } \\
\text { (STAND- }\end{array}$ & $\begin{array}{l}\text { TEMPER - } \\
\text { ATURE }\end{array}$ & $\begin{array}{c}\text { TEMPER - } \\
\text { ATURE }\end{array}$ \\
\hline NUMBER & DATE & TUDE & TUDE & $\begin{array}{l}\text { TOTAL } \\
\text { (FEET) } \\
\text { (72008) }\end{array}$ & $\begin{array}{l}\text { ABOVE } \\
\text { NGVD) } \\
(72000)\end{array}$ & $\begin{array}{c}\text { LAB } \\
\text { (US/CM) } \\
(90095)\end{array}$ & $\begin{array}{r}\text { SOLVED } \\
(M G / L) \\
(70300)\end{array}$ & $\begin{array}{c}\text { ARD } \\
\text { UNITS }) \\
(00400)\end{array}$ & $\begin{array}{c}\text { AIR } \\
(D E G C) \\
(00020)\end{array}$ & $\begin{array}{l}\text { WATER } \\
\text { (DEG C) } \\
(00010)\end{array}$ \\
\hline
\end{tabular}

\begin{tabular}{|c|c|c|c|c|c|c|c|c|c|c|c|}
\hline \multirow[t]{4}{*}{ NHS } & 14 & $08 \cdot 19 \cdot 62$ & $41 \quad 1003$ & 0713313 & $\cdots$ & 30 & 247 & 144 & $\cdots$ & $\cdots$ & $\cdots$ \\
\hline & & $08-23-88$ & & & & & 233 & 130 & 6.1 & 25.0 & 12.0 \\
\hline & & $07-26-89$ & & & & & 252 & $\cdots$ & 6.0 & $-\cdot$ & 12.0 \\
\hline & & $08-27-90$ & & & & & 245 & $\cdots$ & 5.7 & 28.5 & 11.0 \\
\hline \multirow[t]{2}{*}{ NHS } & 19 & $08-20-62$ & 410948 & 0713630 & $\cdots$ & 18 & 191 & 112 & $\cdots$ & $\cdots$ & $\cdots$ \\
\hline & & $08 \cdot 23 \cdot 88$ & & & $\cdots$ & & 182 & 102 & 6.0 & 19.0 & 12.5 \\
\hline \multirow[t]{3}{*}{ NHS } & 88 & $01-25-57$ & 411248 & 0713423 & 3 & 10 & $\cdots$ & $\cdots$ & $\cdots$ & $\cdots$ & $\cdots$ \\
\hline & & $08-18-62$ & & & & & 189 & 106 & $\cdots$ & $-\cdot$ & $\cdots$ \\
\hline & & $08-16-88$ & & & & & 162 & 90 & 6.4 & 27.5 & 21.5 \\
\hline NHS & 109 & $08-29-90$ & 411009 & 0713623 & $\cdots$ & 12 & 154 & $\cdots$ & 6.2 & 24.0 & 18.0 \\
\hline NHS & 132 & $08-28-90$ & 411023 & 0713409 & 6 & 12 & 151 & $\cdots$ & 5.8 & 26.0 & 11.5 \\
\hline \multirow[t]{2}{*}{ NHS } & 146 & $08-21-62$ & 411059 & 0713458 & 12 & 1 & 164 & 99 & $\cdot \cdot$ & $\cdots$ & $\cdots$ \\
\hline & & $08-17-88$ & & & & & 161 & 86 & 6.3 & 26.5 & 13.0 \\
\hline NHS & 164 & $08-23-62$ & 410851 & 0713544 & $\cdots$ & 10 & 165 & 92 & $\cdots$ & $\cdots$ & $\cdots$ \\
\hline NHS & 510 & $08-27-90$ & 410924 & 0713251 & $\cdots$ & 70 & 276 & $\cdots$ & 5.3 & 30.0 & 12.0 \\
\hline \multirow[t]{2}{*}{ NHW } & 11 & $08 \cdot 17 \cdot 62$ & 411031 & 0713414 & 63 & 6 & 1060 & 592 & $\cdots$ & $\cdots$ & $\cdots$ \\
\hline & & $08 \cdot 23 \cdot 88$ & & & & & 1080 & 606 & 7.1 & 26.0 & 14.0 \\
\hline \multirow[t]{3}{*}{ NHW } & 31 & $08-20-62$ & 411011 & 0713447 & 155 & 103 & 182 & 109 & $\cdots$ & -. & $\cdots$ \\
\hline & & $05-23-63$ & & & & & 256 & 160 & $\cdots$ & $\cdots$ & $\cdots$ \\
\hline & & $08 \cdot 19 \cdot 88$ & & & & & 143 & 93 & 6.3 & 19.5 & 21.5 \\
\hline \multirow[t]{3}{*}{ NHW } & 33 & $04-12-63$ & 410931 & 0713403 & 135 & 131 & $\cdots$ & 397 & $\cdots$ & $\cdots$ & -. \\
\hline & & $04-19-63$ & & & & & $\cdots$ & $\cdots$ & $\cdots$ & $\cdots$ & $\cdots$ \\
\hline & & $05-23-63$ & & & & & 284 & 171 & $\cdots$ & $\cdots$ & $\cdots$ \\
\hline \multirow[t]{3}{*}{ NHW } & 34 & $04-12-63$ & 410933 & 0713401 & 107 & 130 & $\cdots$ & 88 & $\cdots$ & $\cdots$ & $\cdots$ \\
\hline & & $04-19-63$ & & & & & $\cdots$ & $\cdots$ & $\cdots$ & $\cdots$ & $\cdots$ \\
\hline & & $05-23-63$ & & & & & 174 & 107 & $\cdots$ & $\cdots$ & $\cdots$ \\
\hline NHW & 35 & $07-15 \cdot 54$ & 411105 & 0713405 & 72 & 9 & $\cdot-$ & $\cdots$ & $\cdots$ & $\cdots$ & $\cdots$ \\
\hline \multirow[t]{4}{*}{ NHW } & 41 & $08-25-62$ & 411029 & 0713408 & 109 & 11 & 243 & 138 & $\cdots$ & $\cdots$ & $\cdots$ \\
\hline & & $05-24 \cdot 63$ & & & & & $\ddot{i}$ & $\cdots$ & $\cdots$ & $\cdots$ & $\cdots$ \\
\hline & & $08-24-88$ & & & & & 255 & $\cdots$ & 7.1 & $\cdots$ & 17.5 \\
\hline & & $07-28-89$ & & & & & $1_{229}$ & $\cdots$ & 6.4 & $\cdot \cdot$ & 23.0 \\
\hline NHW & 42 & $08-23-88$ & 411026 & 0713342 & 96 & 25 & 259 & 162 & 7.0 & $\cdots$ & 13.0 \\
\hline \multirow[t]{2}{*}{ NHW } & 50 & $08 \cdot 03 \cdot 56$ & 411248 & 0713408 & 233 & 90 & $\cdot \cdot$ & $\cdots$ & $\cdots$ & $\cdots$ & $\cdots$ \\
\hline & & $08-18 \cdot 62$ & & & & & 426 & 224 & $\cdots$ & $\cdots$ & - \\
\hline NHW & 63 & $08-12-57$ & 411325 & 0713343 & 80 & 44 & $\cdots$ & $\cdots$ & $\cdots$ & $\cdots$ & $\cdots$ \\
\hline \multirow[t]{2}{*}{ NHW } & 66 & $09-21-62$ & 410949 & 0713604 & 80 & 88 & 141 & 82 & $\cdots$ & $\cdots$ & $\cdots$ \\
\hline & & $08 \cdot 23 \cdot 88$ & & & & & 106 & 54 & $\cdots$ & 22.0 & 14.0 \\
\hline \multirow[t]{3}{*}{ NHW } & 67 & $09 \cdot 06-60$ & 410926 & 0713300 & 240 & 119 & $\cdots$ & $\cdots$ & $\cdots$ & - & - \\
\hline & & $10-05-62$ & & & & & 441 & 239 & $\cdots$ & $\cdots$ & $\cdots$ \\
\hline & & $05-25-63$ & & & & & $\cdots$ & $\cdots$ & $\cdots$ & $\cdots$ & $\cdots$ \\
\hline
\end{tabular}


water from selected wells and springs

WATER WH, whole water (unfiltered) sample;

fixed-endpoint titration for alkalinity;

Data Storage and Retrieval System code]

\begin{tabular}{|c|c|c|c|c|c|c|c|c|c|c|c|c|}
\hline & & & & & & BICAR- & ALKA- & & & & & \\
\hline & $\begin{array}{l}\text { HARD - } \\
\text { NESS }\end{array}$ & CALCIUM & $\begin{array}{c}\text { MAGNE- } \\
\text { SIUM, }\end{array}$ & SODIUI & $\begin{array}{l}\text { POTAS- } \\
\text { SIUM, }\end{array}$ & $\begin{array}{l}\text { BONATE } \\
\text { WATER }\end{array}$ & $\begin{array}{l}\text { LINITY } \\
\text { WAT WH }\end{array}$ & SULFATE & $\begin{array}{l}\text { CHLO- } \\
\text { RIDE, }\end{array}$ & $\begin{array}{l}\text { FLUO- } \\
\text { RIDE, }\end{array}$ & BROMIDE & $\begin{array}{l}\text { SILICA, } \\
\text { DIS. }\end{array}$ \\
\hline OXYGEN, & TOTAL & DIS- & Is. & DIS & DIs- & WH IT & TOT FET & DIs. & DIS. & DIS- & DIS- & SOLVED \\
\hline DIS- & (MG $/ L$ & SOLVED & SOLVED & SOLVED & SOLVED & LAB & LAB & SOLVED & SOLVED & SOLVED & SOLVED & $(M G / L$ \\
\hline SOLVED & AS & (MG/L & (MG/L & (MG/L & (MG/L & MG/L AS & (MG/L AS & (MG/L & (MG/L & (MG/L & (MG/L & AS \\
\hline$(M G / L)$ & (ACO3) & AS (A) & AS MG) & AS NA) & AS $\mathrm{K}$ ) & $\mathrm{HCO} 3$ & (ACO3) & AS $\mathrm{SO}_{4}$ ) & AS $(L)$ & AS F) & AS BR) & s(02) \\
\hline
\end{tabular}

\begin{tabular}{|c|c|c|c|c|c|c|c|c|c|c|c|c|}
\hline$\cdots$ & 47 & $\cdots$ & $\cdots$ & $\cdots$ & $\cdots$ & $\cdots$ & $\cdots$ & $\cdots$ & 39 & $\cdots$ & $\cdots$ & $\cdots$ \\
\hline$\cdots$ & 40 & 6.7 & 5.7 & 27 & 2.8 & 18 & 16 & 20 & 42 & $\cdots$ & 0.16 & 12 \\
\hline -. & - & -. & .. & -. & -. & $\cdots$ & .. & $\cdots$ & 41 & $\cdots$ & -. & $-\cdot$ \\
\hline 9.0 & -. & -. & $\cdots$ & -. & - & 16 & 13 & $\cdots$ & $\cdots$ & $\cdots$ & -. & $\cdots$ \\
\hline$\cdots$ & 34 & -- & $\cdots$ & -. & -- & $\cdots$ & $\cdots$ & $\cdots$ & 34 & $\cdots$ & -. & $\cdots$ \\
\hline -. & -. & -. & .. & -. & 2.0 & 18 & 15 & 16 & 30 & -. & .10 & . \\
\hline$\cdots$ & $\cdots$ & -- & -. & -. & -. & $\cdots$ & $\cdots$ & $\cdots$ & 36 & $\cdots$ & $\cdots$ & $\cdots$ \\
\hline$\cdots$ & 32 & - & $\cdots$ & .. & -. & - & -. & $\cdots$ & 34 & $\cdots$ & -. & - \\
\hline$\cdots$ & 30 & 4.6 & 4.5 & 18 & 1.1 & 21 & 19 & 19 & 23 & - & .10 & 11 \\
\hline$\cdots$ & 27 & 4.9 & 3.5 & 17 & 1.5 & 19 & 15 & 16 & 16 & 0.2 & .09 & 17 \\
\hline 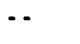 & 27 & 4.3 & 3.9 & 16 & 1.4 & 16 & 11 & 15 & 26 & $<.1$ & $\cdots$ & 13 \\
\hline$\cdots$ & 36 & - & - & -. & -. & $\cdots$ & - & $-\cdot$ & 27 & -. & -. & -. \\
\hline .. & 31 & 5.5 & 4.2 & 17 & 1.7 & 16 & 15 & 12 & 22 & - & .08 & 13 \\
\hline$\cdots$ & 22 & $\cdots$ & $\cdots$ & $\cdots$ & $\cdots$ & $\cdots$ & - & - & 32 & $\cdots$ & $\cdots$ & $\cdots$ \\
\hline$\cdots$ & $\cdots$ & $\cdots$ & $\cdots$ & $\cdot \cdot$ & -. & 20 & 15 & -. & -. & -. & -. & $\cdots$ \\
\hline$\cdots$ & 110 & - & $\cdots$ & -. & - & -. & - & $\cdots$ & 300 & - & $\cdots$ & $\cdots$ \\
\hline$\cdots$ & 110 & 19 & 15 & 170 & 5.3 & 58 & 50 & 43 & 300 & - & 1.1 & 22 \\
\hline$\cdots$ & 33 & $\cdots$ & - & $\cdots$ & - & -. & -. & - & 24 & - & - & $\cdots$ \\
\hline$\cdots$ & 86 & - & $\cdots$ & -. & -. & $\cdots$ & -. & -. & 23 & - & -. & $\cdots$ \\
\hline$\cdots$ & - & $\cdots$ & $\cdots$ & - & 1.0 & 27 & 23 & 17 & 15 & - & .09 & $\cdots$ \\
\hline$\cdots$ & 54 & $\cdots$ & -. & -. & - & $\cdots$ & - & -. & 34 & - & -. & .. \\
\hline$\cdots$ & -. & $\cdots$ & -. & .. & -- & -. & -. & -. & 40 & -. & $\cdots$ & $\cdots$ \\
\hline$\cdots$ & 55 & - & $\cdots$ & - & $-\cdot$ & $\cdots$ & - & -. & 41 & - & $\cdots$ & $\cdots$ \\
\hline$\cdots$ & 30 & -. & $\cdots$ & -. & -. & $\cdots$ & -. & .. & 28 & - & -. & $\cdots$ \\
\hline$\cdots$ & $-\cdot$ & $-\cdot$ & $\cdots$ & $\cdots$ & $-\cdot$ & $\cdots$ & - & $\cdots$ & 31 & - & -. & $\cdots$ \\
\hline$\cdots$ & 28 & - & -. & - & - & $\cdots$ & $\cdots$ & -. & 28 & $-\cdot$ & $\cdots$ & $\cdots$ \\
\hline$\cdots$ & $\cdots$ & $\cdots$ & $\cdot \cdot$ & $\cdots$ & $-\cdot$ & $\cdots$ & $-\cdot$ & $\cdots$ & 2000 & - & $\cdots$ & $\cdots$ \\
\hline$\cdots$ & 28 & $\cdots$ & -. & - & - & $\cdots$ & - & -. & 51 & - & -. & $\cdots$ \\
\hline$\cdots$ & - & $\cdots$ & $-\cdot$ & -. & - & $\cdots$ & -. & -- & -. & - & $\cdots$ & $\cdots$ \\
\hline$\cdots$ & $-\cdot$ & $\cdots$ & $\cdot-$ & - & $\cdots$ & 58 & 50 & -. & $\cdots$ & - & -. & $\cdots$ \\
\hline$\cdots$ & - & - & -. & $\cdots$ & $-\cdot$ & $\cdots$ & - & $\cdots$ & 28 & - & . & $\cdots$ \\
\hline$\cdots$ & 48 & 11 & 4.9 & 33 & 2.2 & 57 & 49 & 22 & 47 & -. & .23 & 22 \\
\hline$\cdots$ & $\cdots$ & $\cdots$ & $\cdots$ & $\cdots$ & - & $\cdots$ & -. & $\cdots$ & 52 & - & $\cdots$ & $\cdots$ \\
\hline$\cdots$ & 38 & $\cdots$ & $\cdots$ & $\cdots$ & -. & $\cdots$ & .. & $\cdots$ & 93 & $\cdots$ & $\cdots$ & $\cdots$ \\
\hline$\cdots$ & $\cdots$ & $\cdots$ & $\cdots$ & $\cdots$ & $-\cdot$ & $\cdots$ & $-\cdot$ & $\cdots$ & 1000 & - & $\cdots$ & $\cdots$ \\
\hline$-\cdot$ & 24 & $\cdots$ & - & $\cdots$ & $\cdots$ & $\cdots$ & - & - & 25 & - & $\cdots$ & $\cdots$ \\
\hline$\cdots$ & 19 & 2.7 & 3.0 & 13 & 1.0 & 11 & 11 & 12 & 17 & - & .06 & 12 \\
\hline -. & $\cdots$ & $\cdots$ & - & $\cdots$ & $\cdot \cdot$ & $\cdots$ & - & -. & 83 & - & $\cdots$ & $\cdots$ \\
\hline$\cdots$ & 44 & $\cdots$ & - & $\cdots$ & $\cdots$ & $\cdot \cdot$ & $\cdots$ & $\cdots$ & 93 & - & $\cdots$ & $\cdots$ \\
\hline$\cdots$ & $\cdot \cdot$ & $\cdots$ & $\cdots$ & $\cdots$ & $\cdots$ & -. & - & -. & $\cdots$ & - & -. & - \\
\hline
\end{tabular}


$[\cdots$, no data available; <, less than; WAT WH or

IT, incremental titration for alkal inity; FET, number in parentheses is USGS National Water

\begin{tabular}{|c|c|c|c|c|c|c|c|c|c|c|c|c|}
\hline \multicolumn{2}{|c|}{$\begin{array}{l}\text { STATION } \\
\text { NUMBER }\end{array}$} & DATE & $\begin{array}{l}\text { NITRO- } \\
\text { GEN, } \\
\text { NI TRATE } \\
\text { TOTAL } \\
\text { (MG/L } \\
\text { AS N) } \\
(00620)\end{array}$ & $\begin{array}{c}\text { NITRO- } \\
\text { GEN, } \\
\text { NITRITE } \\
\text { DIS- } \\
\text { SOLVED } \\
\text { (MG/L } \\
\text { AS N) } \\
(00613)\end{array}$ & $\begin{array}{l}\text { NITRO- } \\
\text { GEN, } \\
\text { NO2+NO3 } \\
\text { DIS- } \\
\text { SOLVED } \\
\text { (MG/L } \\
\text { AS N) } \\
(00631)\end{array}$ & $\begin{array}{l}\text { NITRO- } \\
\text { GEN, } \\
\text { AMMONIA } \\
\text { DIS- } \\
\text { SOLVED } \\
\text { (MG/L } \\
\text { AS N) } \\
(00608)\end{array}$ & $\begin{array}{l}\text { NITRO- } \\
\text { GEN,AM- } \\
\text { MONIA + } \\
\text { ORGANIC } \\
\text { DIS. } \\
\text { (MG/L } \\
\text { AS N) } \\
(00623)\end{array}$ & $\begin{array}{l}\text { PHOS- } \\
\text { PHORUS } \\
\text { ORTHO, } \\
\text { DIS- } \\
\text { SOLVED } \\
\text { (MG/L } \\
\text { AS P) } \\
(00671)\end{array}$ & $\begin{array}{l}\text { BARIUM, } \\
\text { DIS- } \\
\text { SOLVED } \\
\text { (UG/L } \\
\text { AS BA) } \\
(01005)\end{array}$ & $\begin{array}{l}\text { BERYL- } \\
\text { LIUM, } \\
\text { DIS- } \\
\text { SOLVED } \\
\text { (UG/L } \\
\text { AS BE) } \\
(01010)\end{array}$ & $\begin{array}{l}\text { CADMIUM } \\
\text { DIS- } \\
\text { SOLVED } \\
\text { (UG/L. } \\
\text { AS CD) } \\
(01025)\end{array}$ & $\begin{array}{l}\text { CHRO- } \\
\text { MIUM, } \\
\text { DIS- } \\
\text { SOLVED } \\
\text { (UG/L } \\
\text { AS CR) } \\
(01030)\end{array}$ \\
\hline \multirow[t]{4}{*}{ NHS } & 14 & $08-19-62$ & $\cdots$ & $\cdots$ & $\cdots$ & $\cdots$ & $\therefore$ & $\cdots$ & $\cdots$ & $\cdots$ & $\cdots$ & $\therefore$ \\
\hline & & $08-23-88$ & -. & $\cdots$ & 2.1 & $<0.01$ & 0.5 & -- & 93 & $<0.5$ & $<1$ & $<5$ \\
\hline & & $07-26-89$ & -. & $<0.01$ & 1.9 & $<.01$ & $-\cdot$ & $-\cdot$ & $\cdots$ & -. & $\cdots$ & $\cdots$ \\
\hline & & $08-27-90$ & .. & $-\cdot$ & -. & -. & -. & $\cdots$ & $\cdots$ & -. & -. & $\cdots$ \\
\hline \multirow[t]{2}{*}{ NHS } & 19 & $08-20-62$ & $\cdots$ & $\cdots$ & -. & $\cdots$ & $\cdots$ & $\cdots$ & $\cdots$ & $\cdots$ & $\cdot \cdot$ & $\cdots$ \\
\hline & & $08-23-88$ & -. & $\cdots$ & 1.8 & $<.01$ & .7 & $\cdots$ & -. & -. & -. & \\
\hline \multirow[t]{3}{*}{ NHS } & 88 & $01 \cdot 25 \cdot 57$ & 4.4 & $\cdots$ & $\cdots$ & $-\cdot$ & $\cdots$ & .. & $\cdots$ & $\cdots$ & $\cdots$ & $\cdots$ \\
\hline & & $08-18-62$ & $\cdots$ & $\cdots$ & $\cdots$ & $-\cdot$ & $\cdots$ & $\cdots$ & -. & $\cdots$ & 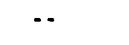 & $\cdots$ \\
\hline & & $08-16-88$ & $-\cdot$ & $\cdots$ & 0.657 & $<.01$ & .4 & $\cdots$ & 19 & $<.5$ & 1 & $<5$ \\
\hline NHS & 109 & $08-29 \cdot 90$ & $\cdots$ & $\cdot \cdot$ & 1.9 & $\cdot \cdot$ & $\cdot \cdot$ & 0.02 & $\cdot \cdot$ & $\cdots$ & $\cdots$ & $\cdots$ \\
\hline NHS & 132 & $08-28-90$ & $\cdots$ & -. & $\cdots$ & -. & -. & $\cdots$ & -. & - & .. & \\
\hline \multirow[t]{2}{*}{ NHS } & 146 & $08-21-62$ & -. & -. & -. & - & $\cdots$ & -. & -. & $\cdots$ & $-\cdot$ & $-\cdot$ \\
\hline & & $08-17-88$ & -. & $\cdots$ & 2.6 & $<.01$ & .4 & - & 110 & $<.5$ & $<1$ & $<5$ \\
\hline NHS & 164 & $08-23-62$ & $\cdots$ & $\cdots$ & $\cdots$ & -. & $\cdot-$ & $\cdots$ & $\cdots$ & -. & $\cdots$ & $\cdots$ \\
\hline NHS & 510 & $08-27-90$ & $\cdots$ & $\cdots$ & $\cdots$ & $\cdots$ & $\cdots$ & $\cdots$ & $\cdots$ & $\cdots$ & $\cdots$ & $\cdots$ \\
\hline \multirow[t]{2}{*}{ NHW } & 11 & $08-17-62$ & -. & -. & $\cdots$ & .. & -. & $\cdots$ & -. & -. & -. & \\
\hline & & $08-23-88$ & -. & $\cdots$ & $<.01$ & .25 & .5 & -. & 79 & $<.5$ & 1 & $<5$ \\
\hline \multirow[t]{3}{*}{ NHW } & 31 & $08-20-62$ & -. & - & $\cdots$ & $\cdots$ & $\cdots$ & $\cdots$ & $\cdots$ & $\cdots$ & $\cdots$ & $\cdots$ \\
\hline & & $05-23-63$ & $\cdots$ & -. & $\cdots$ & $-\cdot$ & $\cdots$ & $\cdots$ & $-\cdot$ & $\cdots$ & -. & $\cdots$ \\
\hline & & $08-19-88$ & $\cdots$ & $\cdots$ & 1.0 & $<.01$ & .2 & $\cdots$ & $\cdots$ & $\cdots$ & $\cdots$ & $\cdots$ \\
\hline \multirow[t]{3}{*}{ NHW } & 33 & $04-12-63$ & $\cdots$ & $\cdots$ & $\cdots$ & $\cdots$ & $-\cdot$ & $\cdots$ & .. & -. & .. & \\
\hline & & $04-19-63$ & $\cdots$ & $\cdots$ & $\cdots$ & $\cdots$ & $\cdots$ & -. & -. & - & -. & $\cdots$ \\
\hline & & $05-23-63$ & $\cdots$ & $\cdots$ & $\cdots$ & $\cdots$ & $\cdots$ & $\cdots$ & $\cdots$ & -. & .. & -. \\
\hline \multirow[t]{3}{*}{ NHW } & 34 & $04-12-63$ & $\cdot-$ & $\cdots$ & $\cdots$ & $\cdots$ & $\cdot \cdot$ & $\cdots$ & $\cdot \cdot$ & $\cdots$ & $\cdot-$ & $\cdots$ \\
\hline & & $04-19-63$ & $\cdots$ & $\cdots$ & $\cdots$ & $\cdots$ & $\cdots$ & $\cdots$ & $\cdots$ & $\cdots$ & -. & $\cdots$ \\
\hline & & $05-23 \cdot 63$ & -. & -. & .. & .. & .. & . & .. & . & .. & \\
\hline NHW & 35 & $07-15-54$ & -- & $-\cdot$ & $-\cdot$ & $\cdots$ & $-\cdot$ & -. & -. & -. & $\cdots$ & $\cdots$ \\
\hline \multirow[t]{4}{*}{ NHW } & 41 & $08 \cdot 25 \cdot 62$ & -. & $-\cdot$ & $-\cdot$ & $\cdots$ & -- & $\cdots$ & -. & -. & $\cdots$ & $\cdots$ \\
\hline & & $05-24-63$ & -. & $\cdots$ & .. & $-\cdot$ & $-\cdot$ & -. & $\cdots$ & $\cdots$ & -. & -. \\
\hline & & $08-24-88$ & $\cdots$ & $\cdots$ & -. & $\cdots$ & $\cdots$ & $\cdots$ & -. & $\cdots$ & $\cdots$ & $\cdots$ \\
\hline & & $07 \cdot 28-89$ & -. & $<.01$ & .02 & .660 & -. & $\cdots$ & -. & $\cdots$ & -. & \\
\hline NHW & 42 & $08-23-88$ & $\cdots$ & $\cdots$ & $<.01$ & .10 & .3 & $\cdots$ & 61 & $<.5$ & $<1$ & $<5$ \\
\hline \multirow[t]{2}{*}{ NHW } & 50 & $08-03-56$ & 0 & $\cdots$ & $\cdot-$ & $\cdots$ & $\cdot \cdot$ & $\cdots$ & $\cdots$ & $\cdots$ & $\cdots$ & $\cdots$ \\
\hline & & $08-18-62$ & -. & $-\cdot$ & -. & $\cdots$ & - & $\cdots$ & -. & $\cdots$ & -. & -- \\
\hline NHW & 63 & $08-12 \cdot 57$ & 3.1 & $\cdots$ & -. & $\cdots$ & $\cdot-$ & $\cdots$ & $\cdots$ & $\cdots$ & $\cdots$ & 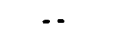 \\
\hline \multirow[t]{2}{*}{ NHW } & 66 & $09-21.62$ & .. & -. & .. & .. & -. & -. & -. & $\cdots$ & -. & \\
\hline & & $08-23-88$ & $\cdots$ & $\cdots$ & .573 & $<.01$ & .2 & $\cdots$ & 30 & $<.5$ & $<1$ & $<5$ \\
\hline \multirow[t]{3}{*}{ NHW } & 67 & $09-06-60$ & 0 & $\cdots$ & $\cdots$ & -. & -. & $\cdots$ & $\cdots$ & $\cdots$ & $-\cdot$ & -. \\
\hline & & $10-05 \cdot 62$ & $\cdot-$ & $\cdots$ & $\cdots$ & $\cdots$ & $\cdots$ & $\cdots$ & $\cdots$ & $\cdots$ & $\cdots$ & $\cdots$ \\
\hline & & $05-25 \cdot 63$ & -. & $\cdots$ & $-\cdot$ & -. & $\cdots$ & $\cdots$ & -. & $\cdots$ & -- & $-\cdot$ \\
\hline
\end{tabular}


water from selected wells and springs--Continued

WATER WH, whole water (unfiltered) sample;

fixed-endpoint titration for alkal inity;

Data Storage and Retrieval System code]

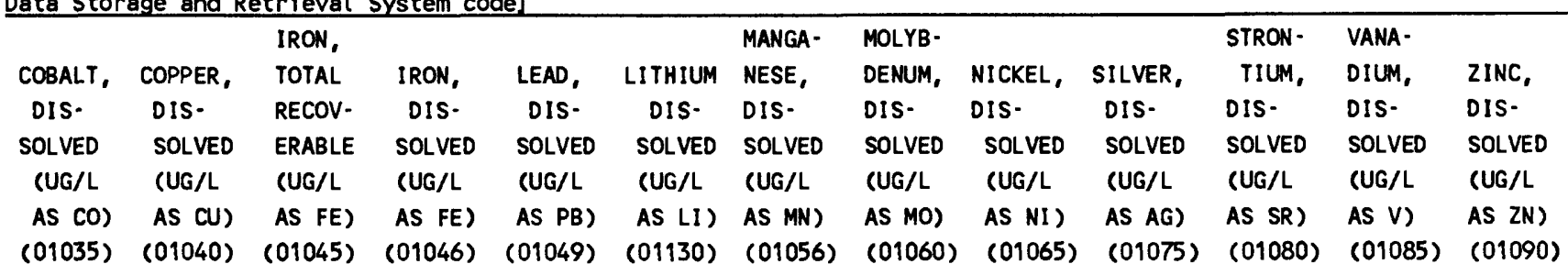

\begin{tabular}{|c|c|c|c|c|c|c|c|c|c|c|c|c|}
\hline$\cdots$ & $\cdots$ & $\cdots$ & 110 & $\cdots$ & $\cdots$ & $\cdots$ & $\cdots$ & $\cdots$ & $\cdots$ & $\cdots$ & $\cdots$ & $\therefore$ \\
\hline$<3$ & $<10$ & -. & 37 & $<10$ & $<4$ & 24 & $<10$ & $<10$ & $<1$ & 64 & $<6$ & $<3$ \\
\hline$\cdots$ & $\cdots$ & -. & 40 & - & -. & 70 & - & $\cdots$ & $\cdots$ & $\cdots$ & $-\cdot$ & $\cdots$ \\
\hline$\cdots$ & $\cdots$ & -. & .. & $\cdots$ & -. & -. & -. & . & $\cdots$ & $\cdots$ & -. & $\cdots$ \\
\hline$\cdots$ & $\cdots$ & $-\cdot$ & 0 & $\cdots$ & $\cdots$ & $\cdots$ & $\cdot-$ & $\cdots$ & $\cdots$ & $\cdots$ & -. & $\cdots$ \\
\hline$\cdots$ & -. & -. & -. & -. & -. & $\cdots$ & .. & $\cdots$ & $\cdots$ & $\cdots$ & .. & $\cdots$ \\
\hline .. & $\cdots$ & - & -. & -. & -. & . & -. & $\cdots$ & $\cdots$ & $\cdots$ & -. & -. \\
\hline$\cdots$ & $\cdots$ & $-\cdot$ & 0 & .. & -. & $\cdots$ & $\cdots$ & $\cdots$ & - & -. & -. & .. \\
\hline$<3$ & 260 & -- & 28 & $<10$ & $<4$ & 4 & $<10$ & $<10$ & 2 & 39 & $<6$ & 43 \\
\hline -. & -. & -- & $<3$ & $\cdots$ & $\cdots$ & 2 & $\cdots$ & $\cdots$ & $\cdots$ & $\cdots$ & -. & $\cdots$ \\
\hline - & - & - & 10 & .. & . & 7 & $\cdots$ & $\cdots$ & - & $\cdots$ & - & . \\
\hline$\cdots$ & $\cdots$ & $\cdots$ & 0 & $\cdots$ & $\cdots$ & $\cdots$ & $\cdots$ & $\cdots$ & -- & - & -. & $\cdots$ \\
\hline$<3$ & $<10$ & -. & 8 & 10 & $<4$ & 3 & $<10$ & $<10$ & 2 & 45 & $<6$ & 23 \\
\hline$\cdots$ & -. & - & 0 & - & -- & $\cdots$ & $\cdots$ & - & -. & -. & -. & $\cdots$ \\
\hline - & -. & $\cdots$ & $\cdots$ & - & - & $\cdots$ & $\cdots$ & $\cdots$ & 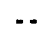 & $\cdots$ & -. & $\cdots$ \\
\hline -. & -. & .. & 0 & - & - & .. & $\cdots$ & $\cdots$ & -. & . & -- & -. \\
\hline$<3$ & $<10$ & -. & 8100 & $<10$ & $<4$ & 920 & $<10$ & $<10$ & $<1$ & 210 & $<6$ & 26 \\
\hline$\cdots$ & -- & -. & 0 & . & .. & .. & -. & $\cdots$ & -. & - & -. & $\cdots$ \\
\hline -. & .. & 10 & $\cdots$ & -. & -. & $\cdots$ & -. & $-\cdot$ & $\cdots$ & $\cdots$ & -. & $\cdots$ \\
\hline-- & - & $\cdots$ & $\cdots$ & - & - & $\cdots$ & $\cdots$ & $\cdots$ & - & $\cdots$ & - & $\cdots$ \\
\hline - & $\cdots$ & 4000 & - & - & - & $\cdots$ & $\cdots$ & .. & -. & -. & - & . \\
\hline -. & $\cdots$ & 5800 & $\cdots$ & -. & -. & $\cdots$ & -. & -. & -. & $\cdots$ & -. & $\cdots$ \\
\hline -. & $\cdots$ & 3000 & -. & -. & -. & $\cdots$ & - & $\cdots$ & $\cdots$ & $\cdots$ & -. & $\cdots$ \\
\hline -. & - & 240 & $-\cdot$ & -. & -- & $\cdots$ & $\cdots$ & $\cdots$ & -. & $\cdots$ & -. & $\cdots$ \\
\hline$\cdots$ & $\cdots$ & 240 & $\cdots$ & $\cdots$ & $\cdots$ & $\cdots$ & $\cdots$ & $\cdots$ & $\cdots$ & - & $\cdots$ & $\cdots$ \\
\hline$\cdots$ & $\cdots$ & 280 & $\cdots$ & .. & $-\cdot$ & $\cdots$ & $\cdots$ & $\cdots$ & -. & - & - & . \\
\hline$\cdots$ & -. & 100 & -. & . & -. & -. & $\cdots$ & $\cdots$ & .. & -. & . & $\cdots$ \\
\hline$\cdots$ & $\cdots$ & $\cdots$ & 70 & - & -. & $\cdots$ & $\cdots$ & $\cdots$ & $\cdots$ & $\cdots$ & - & $\cdots$ \\
\hline$\cdots$ & $\cdots$ & 3000 & $\cdots$ & -. & .. & $\cdots$ & $\cdots$ & $\cdots$ & -. & -. & -. & $\cdots$ \\
\hline$\cdots$ & $\cdots$ & - & $\cdots$ & $\cdots$ & -. & $\cdots$ & $\cdots$ & $\cdots$ & $\cdots$ & $\cdots$ & $-\cdot$ & $\cdots$ \\
\hline -. & $\cdots$ & .. & 11000 & - & - & 450 & - & -. & $\cdots$ & -. & - & $\cdots$ \\
\hline$<3$ & $<10$ & .. & 6200 & $<10$ & $<4$ & 1200 & $<10$ & $<10$ & $<1$ & 96 & $<6$ & 10 \\
\hline$\cdots$ & $\cdots$ & 2800 & - & - & $\cdots$ & $\cdots$ & $-\cdot$ & $\cdots$ & $\cdots$ & $\cdots$ & -. & $\cdots$ \\
\hline$\cdots$ & $\cdots$ & .. & 0 & $\cdots$ & $\cdots$ & $\cdots$ & -. & $\cdots$ & $\cdots$ & -. & -. & - \\
\hline$\cdots$ & $\cdots$ & $\cdots$ & $\cdots$ & $\cdots$ & $\cdots$ & $\cdots$ & $\cdots$ & $-\cdot$ & $\cdots$ & -. & -. & $\cdots$ \\
\hline -. & $\cdots$ & .. & 0 & - & -. & $-\cdot$ & -. & $\cdots$ & $\cdots$ & $\cdots$ & -. & $\cdots$ \\
\hline$<3$ & 50 & $\cdots$ & 95 & $<10$ & $<4$ & 8 & $<10$ & $<10$ & $<1$ & 26 & $<6$ & 29 \\
\hline$\cdots$ & $\cdots$ & - & $\cdots$ & $\cdots$ & $\cdots$ & $\cdots$ & $\cdots$ & $\cdots$ & $\cdots$ & $\cdot$ & -. & $\cdots$ \\
\hline$\cdots$ & $\cdots$ & $\cdots$ & 20 & $\cdots$ & $\cdots$ & $\cdots$ & $\cdots$ & $\cdots$ & $\cdots$ & $\cdots$ & -. & $\cdots$ \\
\hline$\cdots$ & $\cdots$ & 2900 & $\cdots$ & $\cdots$ & $\cdots$ & $\cdots$ & - & $\cdots$ & $\cdots$ & $\cdots$ & $\cdots$ & $\cdot \cdot$ \\
\hline
\end{tabular}


Table 8.--Chemical and physical analyses of

[ $\cdots$, no data available; <, less than; WAT WH or

$I T$, incremental titration for alkal inity; FET,

number in parentheses is USGS National Water

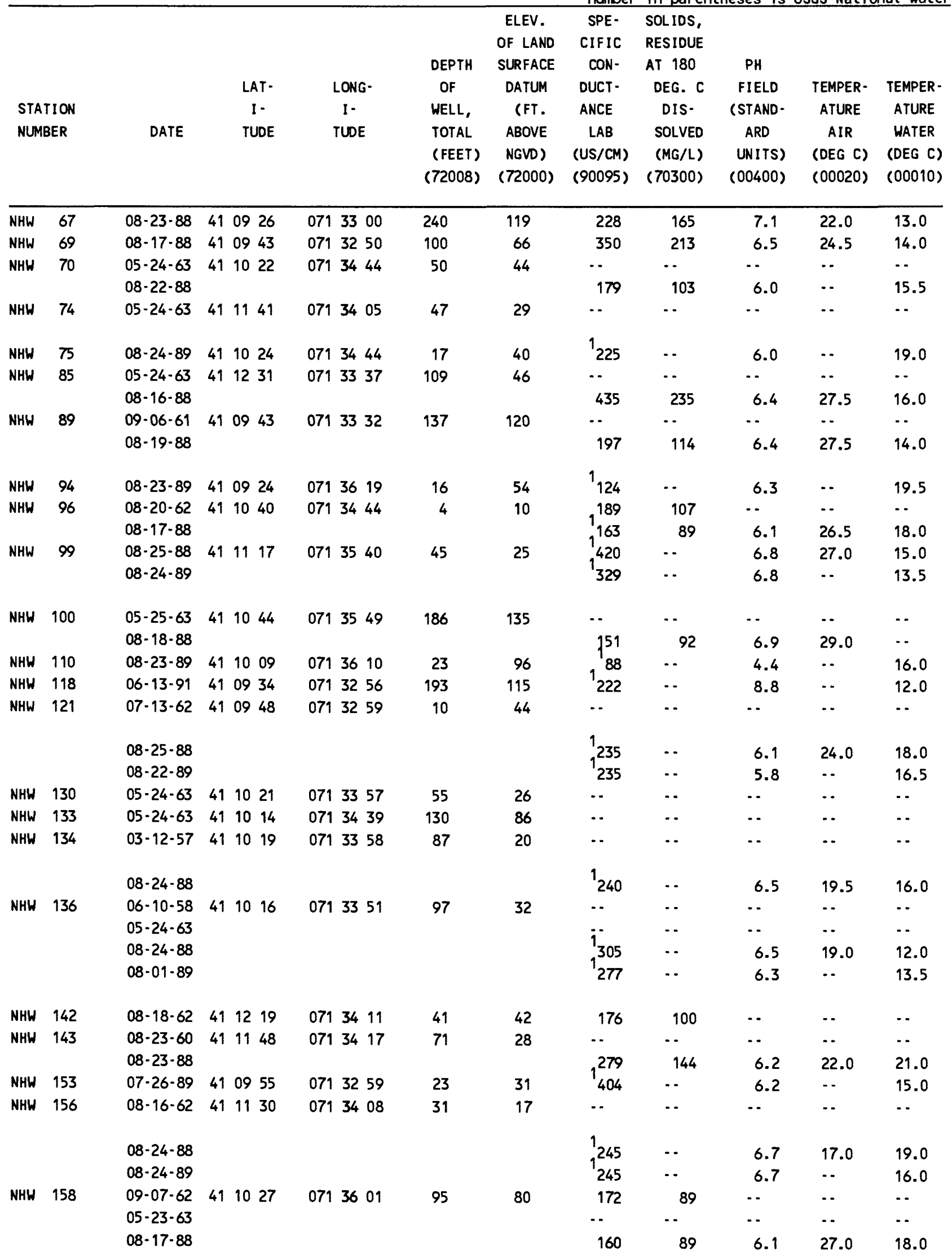


WATER WH, whole water (unfiltered) sample;

fixed-endpoint titration for alkal inity;

Data Storage and Retrieval System code]

\begin{tabular}{|c|c|c|c|c|c|c|c|c|c|c|c|c|}
\hline & & & & & & BICAR- & ALKA- & & & & & \\
\hline & $\begin{array}{l}\text { HARD - } \\
\text { NESS }\end{array}$ & CALCIUM & $\begin{array}{l}\text { MAGNE- } \\
\text { SIUM, }\end{array}$ & SODIUM & $\begin{array}{l}\text { POTAS- } \\
\text { SIUM, }\end{array}$ & $\begin{array}{r}\text { BONATE } \\
\text { WATER }\end{array}$ & $\begin{array}{l}\text { LINITY } \\
\text { WAT WH }\end{array}$ & ATE & $\begin{array}{l}\text { CHLO- } \\
\text { RIDE }\end{array}$ & $\begin{array}{l}\text { FLUO- } \\
\text { RIDE, }\end{array}$ & BROMIDE & $\begin{array}{l}\text { SILICA, } \\
\text { DIS. }\end{array}$ \\
\hline OXYGEN & TOTAL & DIS- & DIS- & DIS- & DIS- & WH IT & LAB & DIS- & DIS. & DIS. & DIS- & SOLVED \\
\hline $\begin{array}{l}\text { DIS- } \\
\text { SOLVED }\end{array}$ & $\begin{array}{c}\text { (MG/L } \\
\text { AS }\end{array}$ & $\begin{array}{l}\text { SOLVED } \\
\text { (MG/L }\end{array}$ & $\begin{array}{l}\text { SOLVED } \\
\text { (MG/L }\end{array}$ & $\begin{array}{c}\text { SOLVED } \\
\text { (MG/L }\end{array}$ & $\begin{array}{l}\text { SOLVED } \\
\text { (MG/L }\end{array}$ & $\begin{array}{c}\text { LAB } \\
M G / L \text { AS }\end{array}$ & $\begin{array}{c}\text { (MG/L } \\
\text { AS }\end{array}$ & $\begin{array}{l}\text { SOLVED } \\
\text { (MG/L }\end{array}$ & $\begin{array}{l}\text { SOLVED } \\
\text { (MG/L }\end{array}$ & $\begin{array}{l}\text { SOLVED } \\
\text { (MG/L }\end{array}$ & $\begin{array}{l}\text { SOLVED } \\
\text { (MG/L }\end{array}$ & $\begin{array}{l}\text { (MG/L } \\
\text { AS }\end{array}$ \\
\hline$(M G / L)$ & $(A C O 3)$ & AS (A) & AS MG) & AS NA) & AS $\mathrm{K}$ ) & $\mathrm{HCO} 3$ & (ACO3) & AS $\mathrm{SO}_{4}$ ) & AS $(L)$ ) & AS $\mathrm{F}$ ) & AS BR) & S(02) \\
\hline$(00300)$ & $(00900)$ & $(00915)$ & $(00925)$ & $(00930)$ & $(00935)$ & $(00449)$ & $(90410)$ & $(00945)$ & $(00940)$ & $(00950)$ & $(71870)$ & $(00955)$ \\
\hline
\end{tabular}

\begin{tabular}{|c|c|c|c|c|c|c|c|c|c|c|c|c|}
\hline$\cdots$ & 44 & 8.3 & 5.6 & 28 & 1.9 & 65 & 54 & 28 & 42 & $\cdots$ & 0.41 & 23 \\
\hline$\cdots$ & 54 & 10 & 6.9 & 40 & 2.2 & 38 & 31 & 45 & 60 & - & .45 & 22 \\
\hline$\cdots$ & $\cdots$ & $\cdots$ & - & $\cdots$ & -. & $\cdots$ & $\cdots$ & .. & .. & $\cdots$ & $\cdots$ & $\cdots$ \\
\hline$\cdots$ & -. & - & - & .. & 2.0 & 21 & 18 & 17 & 23 & - & .098 & $\cdots$ \\
\hline$\cdots$ & $\cdots$ & $\cdots$ & - & -. & -. & - & $\cdots$ & $\cdots$ & $\cdots$ & - & $\cdots$ & $\cdots$ \\
\hline$\cdots$ & -. & .. & - & $\cdots$ & -. & -. & -. & $\cdots$ & 20 & -. & -. & $\cdots$ \\
\hline$\cdots$ & -. & $\cdots$ & - & $\cdots$ & - & $\cdots$ & $\cdots$ & $\cdots$ & $\cdots$ & -. & $\cdots$ & $\cdots$ \\
\hline$\cdots$ & 46 & 5.8 & 7.6 & 57 & 2.9 & 19 & 17 & 23 & 100 & .. & .36 & 15 \\
\hline$\cdots$ & $\cdots$ & $\cdots$ & - & -. & -. & $\cdots$ & $\cdots$ & -. & 32 & - & $\cdots$ & $\cdots$ \\
\hline$\cdots$ & $\cdot-$ & - & $\cdots$ & $\cdots$ & 1.8 & 15 & 13 & 15 & 28 & . & .15 & $\cdots$ \\
\hline
\end{tabular}

\begin{tabular}{|c|c|c|c|c|c|c|c|c|c|c|c|c|}
\hline$\cdots$ & - & -. & $\cdots$ & .. & - & - & . & $\cdots$ & 8.0 & - & .. & - \\
\hline- & 37 & $\cdots$ & -. & $\cdots$ & .. & - & $\cdots$ & $\cdots$ & 28 & $\cdots$ & .. & $\cdots$ \\
\hline$\cdots$ & 32 & 5.7 & 4.2 & 17 & 1.9 & 19 & 19 & 15 & 23 & $\cdots$ & .098 & 14 \\
\hline - & $\cdots$ & $\cdots$ & $\cdots$ & $\cdots$ & $\cdots$ & 117 & 96 & $\cdots$ & $\cdots$ & $\cdots$ & $\cdots$ & - \\
\hline - & $\cdots$ & $\cdots$ & - & -. & $\cdots$ & $\cdots$ & - & $\cdots$ & 43 & $\cdots$ & -. & -. \\
\hline
\end{tabular}


$[\cdots$, no data available; <, less than; WAT WH or

$I T$, incremental titration for alkal inity; FET,

number in parentheses is USGS National Water

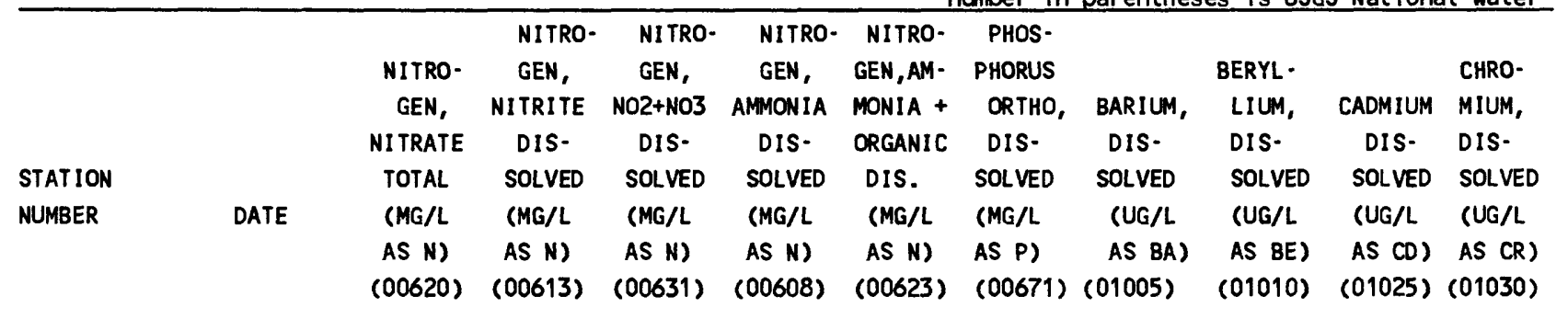

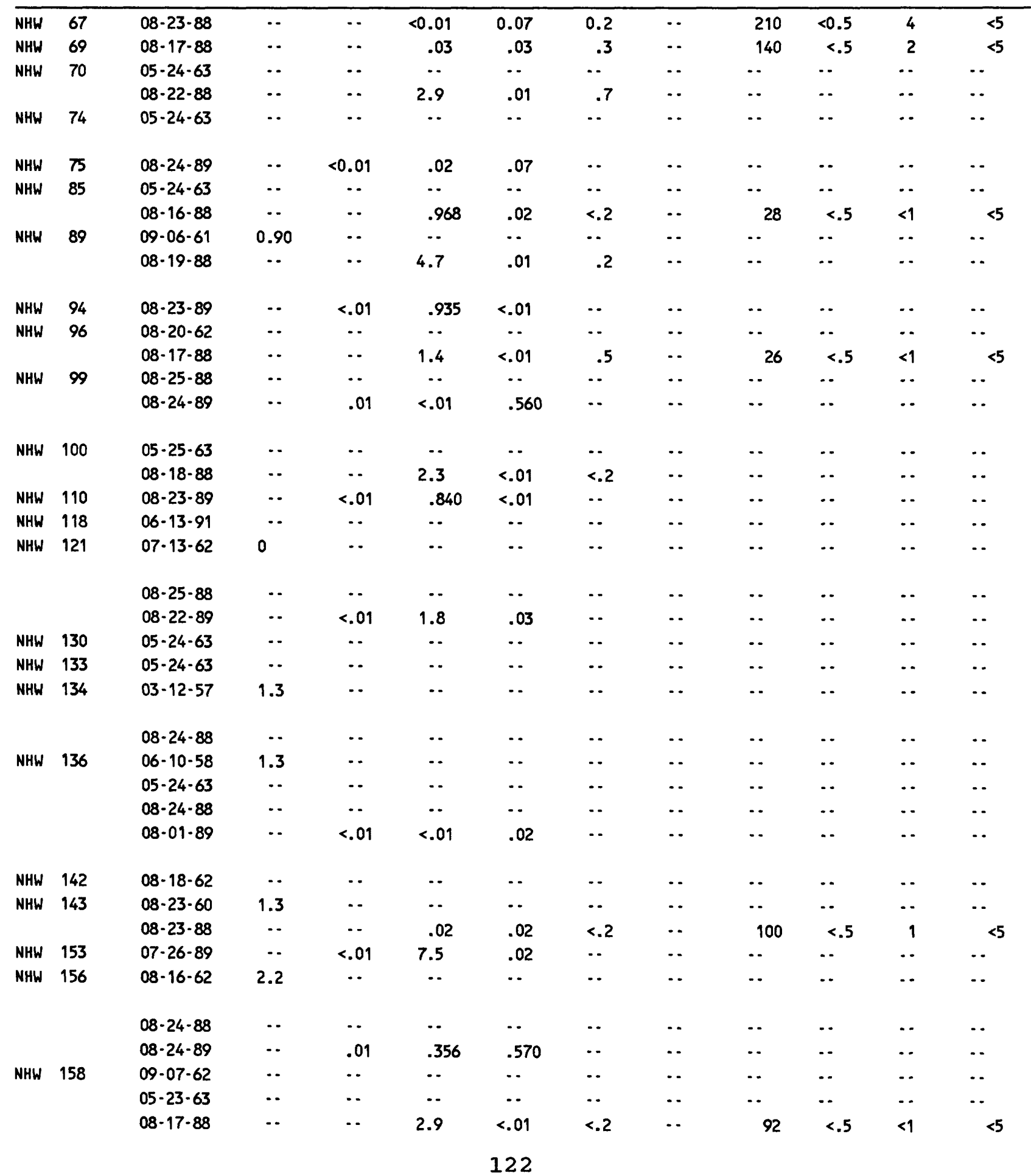


WATER WH, whole water (unfiltered) sample;

fixed-endpoint titration for alkal inity;

Data Storage and Retrieval System code]

\begin{tabular}{|c|c|c|c|c|c|c|c|c|c|c|c|c|}
\hline $\begin{array}{c}\text { COBALT, } \\
\text { DIS- }\end{array}$ & $\begin{array}{c}\text { COPPER, } \\
\text { DIS- }\end{array}$ & IRON, & ON, & $A D$, & 1 & $\begin{array}{l}\text { MANGA- } \\
\text { NESE, }\end{array}$ & $\begin{array}{l}\text { MOLYB- } \\
\text { DENUM, }\end{array}$ & , & ? & $\begin{array}{c}\text { STRON- } \\
\text { TIUM, } \\
\text { DIS- }\end{array}$ & $\begin{array}{l}\text { VANA- } \\
\text { DIUM, }\end{array}$ & c, \\
\hline SOLVE & SOLVED & $\mathrm{E}$ & SOLVED & & & so & SOLVED & sc & & SOLVED & & tE \\
\hline (UG/L & (UG/1 & (U & (UG/L & & & & (UG/L & & & a & & $G / L$ \\
\hline & & & & & & & & & & & & ZN) \\
\hline$(01035)$ & $(01040)$ & 01045) & $01046)$ & 01049) & $(01130)$ & (01056) & $01060)$ & $(01065)$ & $(01075)$ & $(01080)$ & $(01085)$ & $(01090)$ \\
\hline
\end{tabular}

\begin{tabular}{|c|c|c|c|c|c|c|c|c|c|c|c|c|}
\hline$<3$ & $<10$ & $\cdots$ & 16000 & $<10$ & 6 & 670 & $<10$ & $<10$ & $<1$ & 69 & $<6$ & 46 \\
\hline$<3$ & $<10$ & - & 7700 & $<10$ & 5 & 720 & $<10$ & $<10$ & 2 & 74 & $<6$ & 23 \\
\hline$\cdots$ & -. & 60 & -. & -. & -. & $\cdots$ & $\cdots$ & -. & .. & -. & $\cdots$ & .. \\
\hline .. & -. & .. & .. & -. & .. & .. & $\cdots$ & -. & -. & -. & .. & .. \\
\hline$\cdots$ & -. & 90 & -. & $\cdot \cdot$ & $\cdots$ & $\cdots$ & $\cdots$ & -. & $\cdots$ & -. & $\cdots$ & .. \\
\hline$\cdots$ & $-\cdot$ & -. & 20 & $\cdots$ & $\cdots$ & 10 & $\cdots$ & -. & . & .. & $\cdots$ &. \\
\hline$\cdots$ & $\cdot \cdot$ & 80 & .. & $\cdots$ & $\cdots$ & $\cdots$ & .. & -. & .. & .. & .. & .. \\
\hline$<3$ & 100 & $\cdots$ & 8 & $<10$ & $<4$ & 97 & $<10$ & $<10$ & $<1$ & 89 & $<6$ & 1600 \\
\hline$\cdots$ & -. & $\cdots$ & -. & -. & $\cdots$ & .. & $\cdots$ & $\cdots$ & -. & -. & $\cdots$ & .. \\
\hline$\cdots$ & $\cdots$ & $\cdots$ & - & $\cdot \cdot$ & $\cdots$ & $\cdot \cdot$ & $\cdots$ & $\cdot \cdot$ & $\cdots$ & -. & $\cdots$ & .. \\
\hline$\cdots$ & $\ldots$ & .. & 10 & .. & .. & 20 & .. & $\ldots$ & .. & .. & .. & -. \\
\hline$\cdots$ & $\cdots$ & .. & 0 & -. & $\cdots$ & .. & .. & .. & .. & .. & .. & -. \\
\hline$<3$ & 30 & .. & 13 & 10 & $<4$ & 23 & $<10$ & $<10$ & 2 & 50 & $<6$ & 27 \\
\hline$\cdots$ & -. & .. & .. & - & $\cdots$ & .. & $\cdots$ & .. & $\cdots$ & .. & -. & .. \\
\hline$\cdots$ & -. & -. & 27000 & $\cdot$ & $\cdots$ & 430 & 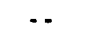 & -. & -. & .. & $\cdots$ & .. \\
\hline .. & -. & 160 & .. & .. & .. & .. & .. & .. & .. & .. & .. & .. \\
\hline -. & .. & $\cdots$ & $\cdots$ & .. & .. & .. & -. & .. & .. & .. & .. & .. \\
\hline$\cdots$ & $\cdots$ & .. & 10 & . & -. & 100 & .. & .. & -. & -. & -. & .. \\
\hline -. & $\cdots$ & $\cdots$ & .. & - & $\cdots$ & .. & .. & -. & -. & $\cdot \cdot$ & .. & .. \\
\hline$\cdots$ & $\cdots$ & $\cdots$ & .. & $\cdots$ & .. & $\cdots$ & . & .. & $\cdots$ & $\cdots$ & $\cdot \cdot$ & -- \\
\hline .. & .. & -. & .. & .. & .. & .. & .. & .. & .. & .. & .. & .. \\
\hline .. & .. & .. & 20 & .. & .. & 20 & .. & .. & .. & .. & .. & .. \\
\hline$\cdots$ & $\cdots$ & 3200 & .. & - & .. & -. & -. & .. & . & .. & .. & .. \\
\hline$\cdots$ & $\cdots$ & 90 & -. & $\cdots$ & $\cdots$ & .. & .. & .. & .. & .. & .. & .. \\
\hline$\cdots$ & $\cdots$ & 1700 & . & $\cdots$ & $\cdots$ & .. & .. & -. & .. & $\cdots$ & -. & .- \\
\hline$\cdots$ & .. & .. & .. & . & .. & .. & -. & .. & . & .. & .. & .. \\
\hline$\cdots$ & $\cdots$ & 11000 & .. & $\cdots$ & -. & $\cdots$ & - & .. & $\ldots$ & -. & .. & .. \\
\hline$\cdots$ & $\cdots$ & 3200 & -. & $\cdots$ & .. & $\cdots$ & -. & -. & $\cdot \cdot$ & .. & .. & .. \\
\hline$\cdots$ & .. & -. & .. & $\cdot \cdot$ & .. & .. & .. & .. & - & $\cdots$ & $\cdots$ & .. \\
\hline -. & .. & -. & 7200 & -. & $\cdots$ & 230 & .. & .. & $\cdots$ & .. & .. & -. \\
\hline$\cdots$ & $\cdots$ & .. & 0 & $\cdots$ & .. & -. & .. & .. & .. & .. & .. & .. \\
\hline$\cdots$ & $\cdots$ & .. & .. & $\cdots$ & .. & .. & .. & .. & .. & .. & .. & .. \\
\hline$<3$ & 30 & $\cdots$ & 130 & 10 & $<4$ & 12 & $<10$ & $<10$ & $<1$ & 79 & $<6$ & 130 \\
\hline$\cdots$ & .. & -. & 20 & -. & $\cdots$ & $<10$ & .. & .. & .. &  & -. & .. \\
\hline$\cdots$ & $\cdots$ & -. & $\cdots$ & $\cdots$ & $\cdots$ & -. & .. & .. & .. & .. & .. & .. \\
\hline$\ldots$ & .. & .. & .. & .. & .. & .. & .. & .. & .. & .. & .. & .. \\
\hline$\cdots$ & $\cdots$ & .. & 10000 & - & $\cdots$ & 270 & .. & .. & .. & .. & .. & .. \\
\hline$\cdots$ & $\cdot \cdot$ & $\cdots$ & 30 & $\cdots$ & $\cdots$ & $\cdots$ & $\cdots$ & -. & .. & .. & .. & .. \\
\hline$\cdots$ & $\cdot \cdot$ & 140 & $\cdot \cdot$ & -. & $\cdots$ & -. & $\cdots$ & -. & $\cdots$ & .. & -. & .. \\
\hline$<3$ & 130 & -. & 140 & $<10$ & $<4$ & 12 & $<10$ & $<10$ & $<1$ & 43 & $<6$ & 18 \\
\hline
\end{tabular}


Table 8.--Chemical and physical analyses of

$[\cdots$, no data available; <, less than; WAT WH or

IT, incremental titration for alkal inity; FET, number in parentheses is USGS National Water

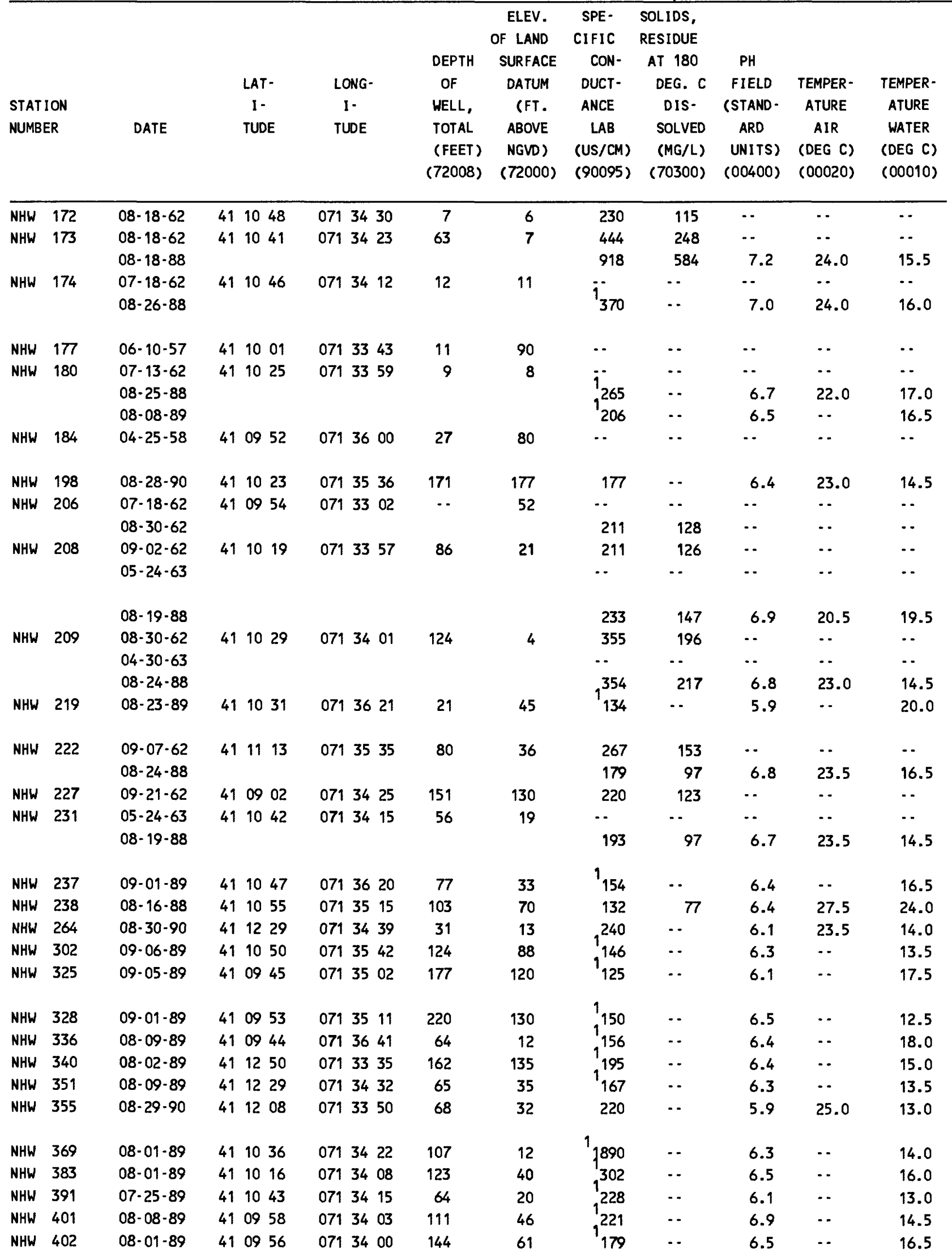


water from selected wells and springs--Continued

WATER WH, whole water (unfiltered) sample;

fixed-endpoint titration for alkalinity;

Data Storage and Retrieval system code]

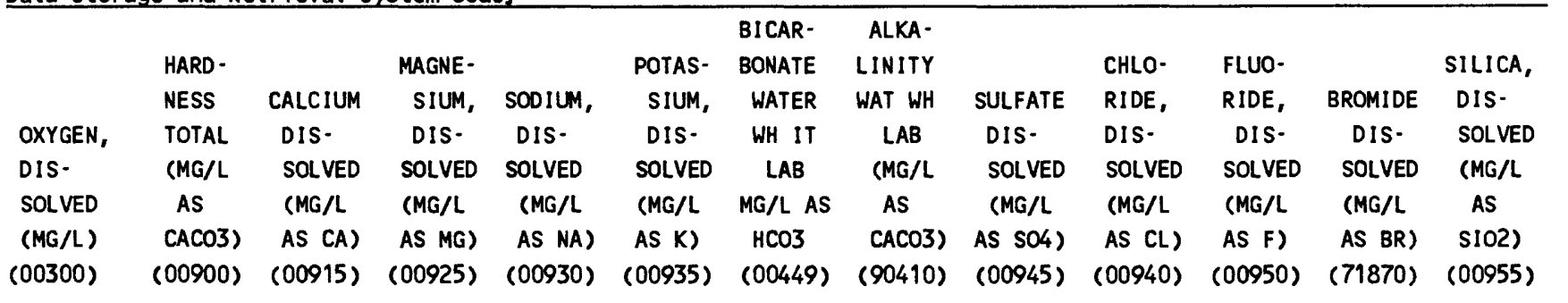

\begin{tabular}{|c|c|c|c|c|c|c|c|c|c|c|c|c|}
\hline$\cdots$ & 46 & $\cdots$ & -. & -. & -. & - & $-\cdot$ & - & 28 & $\cdots$ & - & $\cdots$ \\
\hline$\cdots$ & 28 & $\cdots$ & -. & $\cdots$ & -- & $\cdots$ & $-\cdot$ & - & 110 & -- & -. & $\cdots$ \\
\hline- & -. & -. & -. & -. & 3.5 & 80 & 66 & 7.5 & 260 & - & 1.2 & $\cdots$ \\
\hline$\cdots$ & $\cdots$ & $\cdots$ & $\cdots$ & $\cdots$ & - & - & 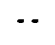 & - & 51 & -. & .. & $\cdots$ \\
\hline$\cdots$ & $\cdots$ & $\cdots$ & $\cdots$ & $\cdots$ & - & 114 & 94 & $\cdots$ & $\cdots$ & $\cdots$ & -. & $\cdots$ \\
\hline$\cdots$ & $\cdots$ & $\cdots$ & - & $\cdots$ & $\cdots$ & - & - & -. & 24 & -. & -. & $\cdots$ \\
\hline- & $\cdots$ & $\cdots$ & - & -. & -- & - & -. & -. & 29 & $\cdots$ & $\cdots$ & $\cdots$ \\
\hline$\cdots$ & $\cdots$ & $\cdots$ & - & $\cdots$ & - & 72 & 60 & $\cdots$ & - & -. & - & $\cdots$ \\
\hline$\cdots$ & $\cdots$ & $\cdots$ & - & $\cdots$ & -. & $\cdots$ & - & $\cdots$ & 11 & -. & -. & - \\
\hline$\cdots$ & $\cdots$ & $\cdots$ & $\cdots$ & $\cdots$ & $\cdots$ & - & $\cdot \cdot$ & $\cdots$ & 26 & $\cdots$ & $\cdot \cdot$ & $\cdots$ \\
\hline$\cdots$ & 33 & 6.1 & 4.2 & 18 & 3.9 & 25 & 20 & 14 & 26 & $<0.1$ & .08 & 19 \\
\hline$\cdots$ & $\cdots$ & $\cdots$ & - & $\cdots$ & - & - & - & - & 39 & $\cdots$ & -. & $\cdots$ \\
\hline$\cdots$ & 40 & - & -. & $\cdots$ & -. & -. & -. & $\cdots$ & 38 & -. & -. & $\cdots$ \\
\hline$\cdots$ & 36 & $\cdots$ & . & -- & $\cdots$ & $\cdots$ & -. & - & 35 & -. & $\cdots$ & $\cdots$ \\
\hline$\cdots$ & $\cdots$ & $\cdots$ & $\cdots$ & $\cdots$ & $\cdots$ & $\cdots$ & - & $\cdots$ & $\cdots$ & $\cdots$ & $\cdots$ & $\cdots$ \\
\hline$\cdots$ & $\because$ & $\cdots$ & . & $\cdots$ & 1.7 & 40 & 34 & 30 & 34 & -. & .25 & $\cdots$ \\
\hline$\cdots$ & 60 & -. & $\cdots$ & $\cdots$ & $\cdots$ & $\cdots$ & -. & - & 61 & $\cdots$ & -. & $\cdots$ \\
\hline$\cdots$ & 110 & $\cdots$ & -. & -. & $\cdots$ & - & -. & -. & 110 & $\cdots$ & -. & $\cdots$ \\
\hline$\cdots$ & 68 & 14 & 7.9 & 40 & 2.5 & 48 & 40 & 48 & 58 & - & .39 & 24 \\
\hline$\cdots$ & $\cdots$ & $\cdots$ & $\cdots$ & - & - & - & $\cdots$ & - & 22 & $\cdots$ & $\cdots$ & - \\
\hline$\cdots$ & 28 & $\cdots$ & $\cdots$ & - & $\cdots$ & - & - & $\cdots$ & 50 & $\cdots$ & .. & $\cdots$ \\
\hline$\cdots$ & 26 & 4.9 & 3.2 & 24 & 1.3 & 32 & 25 & 20 & 25 & - & .14 & 13 \\
\hline$\cdots$ & 28 & $\cdots$ & - & - & - & - & - & - & 40 & - & -. & - \\
\hline$\cdots$ & $\cdots$ & $\cdots$ & -. & $\cdots$ & -. & -. & -. & - & - & $\cdots$ & $\cdots$ & $\cdots$ \\
\hline$\cdots$ & $\cdots$ & $\cdots$ & $\cdots$ & $\cdots$ & 2.1 & 37 & 30 & 17 & 24 & $\cdots$ & .12 & $\cdots$ \\
\hline$\cdots$ & - & $\because$ & $\cdots$ & - - & $\cdots$ & $\cdots$ & - & -. & 20 & $\cdots$ & $\cdots$ & -. \\
\hline$\cdots$ & 25 & 4.5 & 3.3 & 15 & 1.2 & 16 & 16 & 11 & 19 & $\cdots$ & .07 & 15 \\
\hline$\cdots$ & 38 & 5.8 & 5.6 & 28 & 3.0 & 39 & 31 & 11 & 39 & .2 & .15 & 15 \\
\hline$\cdots$ & $\cdots$ & $\cdots$ & -. & $\cdots$ & -. & - & -. & . & 18 & $\cdots$ & $\cdots$ & $\cdots$ \\
\hline$\cdots$ & $\cdots$ & $\cdots$ & - & $\cdots$ & - & - & - & $\cdots$ & 17 & $\cdots$ & -. & $\cdots$ \\
\hline$\cdots$ & $\cdots$ & $\cdots$ & - & -. & $\cdots$ & - & - & - & 21 & $\cdots$ & . & $\cdots$ \\
\hline$\cdots$ & $\cdots$ & $\cdots$ & $\cdots$ & $\cdots$ & -. & - & - & $\cdots$ & 21 & $\cdots$ & $\cdots$ & $\cdots$ \\
\hline$\cdots$ & $\cdots$ & $\cdots$ & -- & $\cdots$ & -- & - & -. & $\cdots$ & - & $\cdots$ & $\cdots$ & $\cdots$ \\
\hline$\cdots$ & $\cdots$ & $\cdots$ & - & $\cdots$ & $\cdots$ & - & - & - & 28 & - & $\cdots$ & $\cdots$ \\
\hline$\cdots$ & 40 & 5.5 & 6.4 & 24 & 1.5 & 21 & 17 & 14 & 34 & .3 & .16 & 13 \\
\hline$\cdots$ & $\cdots$ & $\cdots$ & - & $\cdots$ & - & - & . & $\cdots$ & 540 & $\cdots$ & $\cdots$ & $\cdots$ \\
\hline$\cdots$ & $\cdots$ & $\cdots$ & - & $\cdots$ & - & - & -. & $\cdots$ & 35 & $\cdots$ & $\cdots$ & $\cdots$ \\
\hline$\cdots$ & $\cdots$ & $\cdots$ & $\cdots$ & $\cdots$ & -. & - & -. & $\cdots$ & 30 & $\cdots$ & $\cdots$ & $\cdots$ \\
\hline$\cdots$ & $\cdots$ & $\cdots$ & $\cdots$ & $\cdots$ & $\cdots$ & $\cdots$ & $\cdots$ & $\cdots$ & 29 & $\cdots$ & $\cdots$ & $\cdots$ \\
\hline$\cdots$ & $\cdots$ & $\cdots$ & $\cdots$ & $\cdots$ & - & - & - & $\cdots$ & 23 & $\cdots$ & -. & -. \\
\hline
\end{tabular}


[ - , no data avai lable; <, less than; WAT WH or IT, incremental titration for alkalinity; FET, number in parentheses is National Water

\begin{tabular}{|c|c|c|c|c|c|c|c|c|c|c|c|c|}
\hline \multicolumn{2}{|c|}{$\begin{array}{l}\text { STATION } \\
\text { NUMBER }\end{array}$} & DATE & $\begin{array}{l}\text { NITRO- } \\
\text { GEN, } \\
\text { NITRATE } \\
\text { TOTAL } \\
\text { (MG/L } \\
\text { AS N) } \\
(00620)\end{array}$ & $\begin{array}{l}\text { NITRO- } \\
\text { GEN, } \\
\text { NITRITE } \\
\text { DIS- } \\
\text { SOLVED } \\
\text { (MG/L } \\
\text { AS N) } \\
(00613)\end{array}$ & $\begin{array}{l}\text { NITRO- } \\
\text { GEN, } \\
\text { NO2+NO3 } \\
\text { DIS- } \\
\text { SOLVED } \\
\text { (MG/L } \\
\text { AS N) } \\
(00631)\end{array}$ & $\begin{array}{l}\text { NITRO- } \\
\text { GEN, } \\
\text { AMMONIA } \\
\text { DIS- } \\
\text { SOLVED } \\
\text { (MG/L } \\
\text { AS N) } \\
\text { (00608) }\end{array}$ & $\begin{array}{l}\text { NITRO- } \\
\text { GEN,AM- } \\
\text { MONIA + } \\
\text { ORGANIC } \\
\text { DIS. } \\
\text { (MG/L } \\
\text { AS N) } \\
(00623)\end{array}$ & $\begin{array}{l}\text { PHOS- } \\
\text { PHORUS } \\
\text { ORTHO, } \\
\text { OIS- } \\
\text { SOLVED } \\
\text { (MG/L } \\
\text { AS P) } \\
(00671)\end{array}$ & $\begin{array}{l}\text { BARIUM, } \\
\text { DIS- } \\
\text { SOLVED } \\
\text { (UG/L } \\
\text { AS BA) } \\
(01005)\end{array}$ & $\begin{array}{l}\text { BERYL- } \\
\text { LIUM, } \\
\text { DIS- } \\
\text { SOLVED } \\
\text { (UG/L } \\
\text { AS BE) } \\
\text { (01010) }\end{array}$ & $\begin{array}{l}\text { CADMIUM } \\
\text { DIS- } \\
\text { SOLVED } \\
\text { (UG/L } \\
\text { AS CD) } \\
(01025)\end{array}$ & $\begin{array}{l}\text { CHRO- } \\
\text { MIUM, } \\
\text { DIS- } \\
\text { SOLVED } \\
\text { (UG/L } \\
\text { AS CR) } \\
\text { (01030) }\end{array}$ \\
\hline NHW & 172 & $08-18 \cdot 62$ & $\cdots$ & $\cdots$ & $\cdots$ & $\cdots$ & $\ldots$ & $\cdots$ & $\cdots$ & $\cdots$ & $\cdots$ & $\cdots$ \\
\hline \multirow[t]{2}{*}{ NHW } & 173 & $08 \cdot 18 \cdot 62$ & $\cdots$ & $\cdots$ & $\cdots$ & $\cdots$ & $\cdots$ & $\cdots$ & $\cdots$ & $\cdots$ & $\cdots$ & $\cdots$ \\
\hline & & $08-18-88$ & -. & .. & $<0.01$ & 0.300 & 1.4 & $\cdots$ & .. & $\cdots$ & .. & $\cdots$ \\
\hline \multirow[t]{2}{*}{ NHW } & 174 & $07-18-62$ & 8.9 & $\cdots$ & -. & -. & $\cdots$ & $\cdots$ & -. & $\cdots$ & $\cdots$ & $\cdots$ \\
\hline & & $08-26-88$ & $\cdots$ & $\cdots$ & $\cdots$ & $\cdots$ & $\cdots$ & $\cdots$ & $\cdots$ & $\cdots$ & $\cdots$ & $\cdots$ \\
\hline NHW & 177 & $06-10 \cdot 57$ & 2.2 & $\cdots$ & -. & $\cdots$ & $\cdots$ & .. & $\cdots$ & .. & -. & $\cdots$ \\
\hline \multirow[t]{3}{*}{ NHW } & 180 & $07-13-62$ & 2.2 & $\cdots$ & $\cdots$ & $\cdots$ & $\cdots$ & .. & $\cdots$ & $\cdots$ & $\cdots$ & -. \\
\hline & & $08-25 \cdot 88$ & -. & $\cdots$ & $\cdots$ & $\cdots$ & $\cdots$ & $\cdots$ & $\cdots$ & $\cdots$ & -. & $\cdots$ \\
\hline & & $08-08-89$ & $-\cdot$ & $<0.01$ & 5.1 & .03 & $\cdots$ & $\cdots$ & .. & -. & -. & $\cdots$ \\
\hline NHW & 184 & $04-25-58$ & 1.8 & $\cdots$ & $\cdots$ & $\cdots$ & $\cdots$ & $\cdots$ & $\cdots$ & - & $\cdots$ & $\cdots$ \\
\hline NHW & 198 & $08 \cdot 28-90$ & $\cdots$ & $-\cdot$ & 3.1 & $\cdots$ & $\cdots$ & $<0.01$ & $\cdots$ & .. & -. & .. \\
\hline \multirow[t]{2}{*}{ NHW } & 206 & $07-18-62$ & 0 & $\cdots$ & -. & $\cdots$ & $\cdots$ & .. & $\cdots$ & $\cdots$ & $\cdots$ & $\cdots$ \\
\hline & & $08-30-62$ & -. & $\cdots$ & .. & $\cdots$ & .. & -. & -. & $\cdots$ & .. & -. \\
\hline \multirow[t]{3}{*}{ NHW } & 208 & $09 \cdot 02 \cdot 62$ & $\cdots$ & .. & -. & $\cdots$ & .. & .. & .. & $\ldots$ & .. & $\cdots$ \\
\hline & & $05-24-63$ & $\cdots$ & $\cdots$ & $\cdots$ & $\cdots$ & $\cdots$ & .. & $\cdots$ & $\cdots$ & $\cdots$ & $\cdots$ \\
\hline & & $08-19-88$ & $-\cdot$ & $\cdots$ & $<.01$ & .05 & 0.4 & $\cdots$ & $\cdots$ & .. & - & $\cdots$ \\
\hline \multirow[t]{3}{*}{ NHW } & 209 & $08 \cdot 30 \cdot 62$ & -. & $\cdots$ & -. & $\cdots$ & $\cdots$ & -. & $\cdots$ & $\cdots$ & $\cdots$ & $\cdots$ \\
\hline & & $04-30-63$ & $\cdots$ & $\cdots$ & .. & -. & - & .. & $\cdots$ & -. & -. & $\cdots$ \\
\hline & & $08-24-88$ & -. & -. & $<.01$ & .06 & .2 & .. & 140 & $<0.5$ & $<1$ & $<5$ \\
\hline NHW & 219 & $08-23-89$ & $-\cdot$ & $<.01$ & .044 & .160 & -. & $\ldots$ & $\cdots$ & .. & .. & -. \\
\hline \multirow[t]{2}{*}{ NHW } & 222 & $09 \cdot 07 \cdot 62$ & $\cdots$ & $\cdots$ & -. & $\cdots$ & -. & .. & -. & .. & .. & -. \\
\hline & & $08-24-88$ & $-\cdot$ & -. & .25 & .03 & .3 & $\ldots$ & 120 & $<.5$ & $<1$ & $<5$ \\
\hline NHW & 227 & $09-21 \cdot 62$ & $\cdots$ & $\cdots$ & $\cdots$ & $\cdots$ & $\cdots$ & - & $\cdots$ & -. & .. & .. \\
\hline \multirow[t]{2}{*}{ NHW } & 231 & $05-24-63$ & $\cdots$ & -. & -. & $\cdots$ & $\cdots$ & -. & $\cdots$ & $\cdots$ & $\cdots$ & $\cdots$ \\
\hline & & $08-19-88$ & $\cdots$ & $\cdots$ & 1.8 & $<.01$ & 1.8 & .. & . & .. & $\cdots$ & -. \\
\hline NHW & 237 & 09.01 .89 & .. & $<.01$ & 1.2 & .02 & .. & $\ldots$ & .. & -. & $\ldots$ & $\ldots$ \\
\hline NHW & 238 & $08-16-88$ & $\cdots$ & $\cdots$ & 2.6 & .02 & .6 & -. & 150 & $<.5$ & $<1$ & $<5$ \\
\hline NHW & 264 & $08-30-90$ & -. & $\cdots$ & 1.9 & $\cdots$ & $\cdots$ & .02 & - & -. & .. & .. \\
\hline NHW & 302 & $09 \cdot 06-89$ & $\cdots$ & $<.01$ & 1.3 & .02 & $\cdots$ & .. & .. & .. & $\cdots$ & -. \\
\hline NHW & 325 & $09-05-89$ & $\cdots$ & $<.01$ & 1.7 & .03 & $\cdots$ & -. & $\cdots$ & $\cdots$ & $\cdots$ & $\cdots$ \\
\hline NHW & 328 & $09-01-89$ & $\cdots$ & $<.01$ & 1.1 & .01 & $\cdots$ & .. & -. & - & .. & .. \\
\hline NHW & 336 & $08-09-89$ & $\cdots$ & $<.01$ & .710 & .01 & $-\cdot$ & -. & $\cdots$ & $\cdots$ & $\cdots$ & $\cdots$ \\
\hline NHW & 340 & $08-02-89$ & -. & $<.01$ & .806 & $<.01$ & $\cdots$ & -. & $\cdots$ & .. & $\cdots$ & $\cdots$ \\
\hline NHW & 351 & $08-09.89$ & $\cdots$ & $<.01$ & 3.3 & .02 & $\cdots$ & $\cdots$ & $\cdots$ & $\cdots$ & $\cdots$ & $\cdots$ \\
\hline NHW & 355 & $08 \cdot 29 \cdot 90$ & .. & $-\cdot$ & 1.3 & $\cdots$ & $\cdots$ & .02 & $\cdots$ & $\cdots$ & $\cdots$ & $\cdots$ \\
\hline NHW & 369 & $08-01-89$ & $\cdots$ & $<.01$ & .02 & .290 & .. & -. & - & .. & .. & .. \\
\hline NHW & 383 & $08-01-89$ & $\cdots$ & $<.01$ & $<.01$ & .04 & .. & -. & $\cdots$ & $\cdots$ & $\cdots$ & $\cdots$ \\
\hline NHW & 391 & $07-25-89$ & $\cdots$ & $<.01$ & 2.4 & .04 & $\cdots$ & $\cdots$ & .. & -. & $\cdots$ & -. \\
\hline NHW & 401 & $08-08-89$ & $\cdots$ & $<.01$ & .037 & .490 & $\cdots$ & -. & $\cdots$ & $-\cdot$ & $\cdots$ & $\cdots$ \\
\hline NHW & 402 & $08-01-89$ & $\cdots$ & $<.01$ & .03 & .08 & $\cdots$ & .. & .. & -. & .. & $\ldots$ \\
\hline
\end{tabular}


water from selected wells and springs--Continued

WATER WH, whole water (unfiltered) sample;

fixed-endpoint titration for alkal inity;

Data Storage and Retrieval System code]

$\begin{array}{ccccccccccccc}\text { COBALT, } & \text { COPPER, } & \text { TOTAL } & \text { IRON, } & \text { LEAD, } & \text { LITHIUM } & \text { NESE, } & \text { DENUM, NICKEL, } & \text { SILVER, } & \text { STRON- } & \text { VANA- } \\ \text { TIUM, DIUM, } & \text { ZINC, } \\ \text { DIS- } & \text { DIS- } & \text { RECOV- } & \text { DIS- } & \text { DIS- } & \text { DIS- } & \text { DIS- } & \text { DIS- } & \text { DIS- } & \text { DIS- } & \text { DIS- } & \text { DIS- } & \text { DIS- } \\ \text { SOLVED } & \text { SOLVED } & \text { ERABLE } & \text { SOLVED } & \text { SOLVED } & \text { SOLVED } & \text { SOLVED } & \text { SOLVED } & \text { SOLVED } & \text { SOLVED } & \text { SOLVED } & \text { SOLVED } & \text { SOLVED } \\ (U G / L & (U G / L & (U G / L & (U G / L & (U G / L & (U G / L & (U G / L & (U G / L & (U G / L & (U G / L & (U G / L & (U G / L & (U G / L \\ \text { AS CO } & \text { AS CU) } & \text { AS FE) } & \text { AS FE) } & \text { AS PB) } & \text { AS LI) } & \text { AS MN) } & \text { AS MO) } & \text { AS NI) } & \text { AS AG) } & \text { AS SR) AS V) } & \text { AS ZN) } \\ (01035) & (01040) & (01045) & (01046) & (01049) & (01130) & (01056) & (01060) & (01065) & (01075) & (01080) & (01085) & (01090)\end{array}$

\begin{tabular}{|c|c|c|c|c|c|c|c|c|c|c|c|c|}
\hline$\cdots$ & -. & -. & 0 & - & .. & $\cdots$ & .. & $\ldots$ & $\cdots$ & $\cdots$ & $\cdots$ & $\cdots$ \\
\hline$\cdots$ & -. & $-\cdot$ & 0 & -. & -. & -. & $\cdots$ & $\cdots$ & $\cdots$ & $\cdots$ & $\cdots$ & - \\
\hline$\cdots$ & $\cdots$ & $\cdots$ & -. & - & -. & $\cdots$ & $\cdots$ & $\cdots$ & $\cdots$ & $\cdots$ & $\cdots$ & - \\
\hline$\cdots$ & $\cdots$ & $\cdots$ & $\cdots$ & - & -. & - & -. & $\cdots$ & $\cdots$ & $\cdots$ & $\cdots$ & - \\
\hline$\cdots$ & $\cdot-$ & $-\cdot$ & -- & $\cdots$ & - & $\cdots$ & $\cdots$ & $\cdots$ & $\cdots$ & $\cdots$ & $\cdots$ & $\cdots$ \\
\hline$\cdots$ & $\cdots$ & -. & $\cdots$ & - & -. & $\cdots$ & $\cdots$ & - & $\cdots$ & - & $\cdots$ & .. \\
\hline$\cdots$ & $\cdots$ & $\cdots$ & $\cdots$ & $\cdots$ & -- & $\cdots$ & $\cdots$ & $\cdots$ & $\cdots$ & $\cdots$ & $\cdots$ & $\cdots$ \\
\hline$\cdots$ & $\cdots$ & -. & -. & - & -. & $\cdots$ & $\cdots$ & $\cdots$ & - & $\cdots$ & $\cdots$ & $\cdots$ \\
\hline$\cdots$ & $\cdot \cdot$ & $\cdots$ & 30 & $\cdots$ & $\cdots$ & 50 & $\cdots$ & $\cdots$ & $\cdots$ & $\cdots$ & - & $\cdots$ \\
\hline$\cdots$ & $\cdots$ & $\cdots$ & $\cdot-$ & $\cdots$ & $\cdots$ & $\cdots$ & $\cdots$ & $\cdots$ & $\cdots$ & $\cdots$ & $\cdots$ & $\cdots$ \\
\hline$\cdots$ & $\cdots$ & . & 28 & .. & -. & 5 & - & $\cdots$ & $\cdots$ & $\cdots$ & -. & $\cdots$ \\
\hline$\cdots$ & $\cdots$ & $\cdots$ & $\cdots$ & $\cdots$ & - & - & $\cdots$ & $\cdots$ & $\cdots$ & - & - & $\cdots$ \\
\hline$\cdots$ & $\cdots$ & $\cdots$ & 130 & $\cdots$ & -. & $\cdots$ & $\cdots$ & $\cdots$ & $\cdots$ & - & - & - \\
\hline$\cdots$ & $\cdots$ & - & 80 & 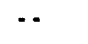 & $\cdots$ & - & $\cdots$ & $\cdots$ & $\cdots$ & $\cdots$ & .. & - \\
\hline$\cdots$ & $\cdots$ & 3000 & $\cdots$ & - & $\cdots$ & $\cdots$ & $\cdots$ & $\cdots$ & $\cdots$ & $\cdots$ & - & $\cdots$ \\
\hline$\cdots$ & $\cdots$ & - & -- & . & - & -. & $\cdots$ & - & $\cdots$ & -. & -. & $\cdots$ \\
\hline$\cdots$ & $\cdots$ & - & 10 & - & .. & -. & $\cdots$ & $\cdots$ & $\cdots$ & - & -. & $\cdots$ \\
\hline$\cdots$ & $\cdots$ & 7000 & $\cdots$ & - & $-\cdot$ & - & -- & - & - & $\cdots$ & -. & $\cdots$ \\
\hline$<3$ & $<10$ & $\cdots$ & 12000 & $<10$ & 5 & 1000 & $<10$ & $<10$ & 1.0 & 100 & $<6$ & 30 \\
\hline$\cdots$ & $\cdots$ & $-\cdot$ & 470 & $\cdots$ & - & 2100 & -- & - & $\cdots$ & $\cdots$ & - & $\cdots$ \\
\hline -. & -. & -. & 10 & - & $\cdots$ & -. & $\cdots$ & - & $\cdots$ & -. & - & -. \\
\hline$<3$ & 20 & $\cdots$ & 240 & $<10$ & $<4$ & 210 & $<10$ & $<10$ & $<1.0$ & 41 & $<6$ & 2000 \\
\hline$\cdots$ & - & $\cdots$ & 0 & $\cdots$ & -. & $\cdots$ & $\cdots$ & $\cdots$ & - & $\cdots$ & -- & $\cdots$ \\
\hline$\cdots$ & $\cdot \cdot$ & 220 & $\cdots$ & $\cdots$ & -. & -. & $\cdots$ & $\cdots$ & $\cdots$ & $\cdots$ & $-\cdot$ & $\cdots$ \\
\hline$\cdots$ & $\cdots$ & $\cdots$ & -. & $-\cdot$ & $\cdots$ & $\cdots$ & $\cdots$ & $\cdots$ & $\cdots$ & $\cdots$ & $\cdots$ & $\cdots$ \\
\hline$\cdots$ & - & $\cdots$ & 70 & - & - & 60 & -. & - & - & $\cdots$ & -. & $\cdots$ \\
\hline$<3$ & 80 & $\cdots$ & 17 & 10 & $<4$ & 6 & $<10$ & $<10$ & 1.0 & 40 & $<6$ & 21 \\
\hline$\cdots$ & $\cdots$ & $\cdots$ & $<3$ & $\cdots$ & -. & 690 & $\cdots$ & $\cdots$ & $\cdots$ & $\cdots$ & $\cdots$ & $\cdots$ \\
\hline$\cdots$ & $\cdots$ & $\cdots$ & 40 & -. & $\cdots$ & 20 & $\cdots$ & $\cdots$ & $\cdots$ & $\cdots$ & $\cdots$ & $\cdots$ \\
\hline$\cdots$ & $\cdots$ & $\cdots$ & 20 & $\cdots$ & $\cdots$ & $<10$ & $\cdot-$ & $\cdots$ & 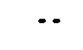 & $\cdots$ & -. & - \\
\hline$\cdots$ & $\cdots$ & $\cdots$ & 70 & - & $\cdots$ & $<10$ & .. & - & $\cdots$ & $\cdots$ & - & $\cdots$ \\
\hline$\cdots$ & $\cdots$ & $\cdots$ & 40 & $\cdots$ & $\cdots$ & 50 & -. & -. & $\cdots$ & $\cdots$ & -. & $\cdots$ \\
\hline$\cdots$ & $\cdots$ & $\cdots$ & 170 & $\cdots$ & -. & 20 & -. & $\cdots$ & $\cdots$ & $\cdots$ & $\cdots$ & $\cdots$ \\
\hline$\cdots$ & $\cdots$ & $\cdots$ & 20 & $\cdots$ & -. & $<10$ & $\cdots$ & $\cdots$ & $\cdots$ & $\cdots$ & -. & $\cdots$ \\
\hline$\cdots$ & $\cdots$ & $\cdots$ & 33 & $\cdots$ & -. & 2 & $\cdots$ & $\cdots$ & - & $\cdots$ & $\cdots$ & - \\
\hline$\cdots$ & $\cdots$ & $\cdots$ & 8600 & $\cdots$ & -. & 3100 & -- & - & $\cdots$ & $\cdots$ & $\cdots$ & .. \\
\hline$\cdots$ & $\cdots$ & $\cdots$ & 10000 & $\cdots$ & -. & 420 & -. & -. & $\cdots$ & - & $\cdots$ & $\cdots$ \\
\hline$\cdots$ & $\cdots$ & $\cdots$ & 60 & -. & $\cdots$ & 30 & $\cdots$ & $\cdots$ & -- & -. & $\cdots$ & -. \\
\hline$-\cdot$ & $\cdots$ & $\cdots$ & 6200 & $\cdots$ & $\cdots$ & 890 & -. & $\cdots$ & -- & - & $\cdots$ & $\cdots$ \\
\hline$\cdots$ & $\cdots$ & $\cdots$ & 3600 & $\cdots$ & $\cdots$ & 530 & -. & -. & -- & - & -. & -. \\
\hline
\end{tabular}


$[\cdots$, no data available; <, less than; WAT WH or

IT, incremental titration for alkalinity; FET, number in parentheses is USGS National Water

\begin{tabular}{|c|c|c|c|c|c|c|c|c|c|c|}
\hline & & & & & $\begin{array}{l}\text { ELEV. } \\
\text { OF LAND }\end{array}$ & $\begin{array}{l}\text { SPE- } \\
\text { CIFIC }\end{array}$ & $\begin{array}{l}\text { SOLIDS, } \\
\text { RESIDUE }\end{array}$ & & & \\
\hline & & & & DEPTH & SURFACE & CON- & AT 180 & & & \\
\hline STAT ION & & $\begin{array}{c}\text { LAT - } \\
\text { I - }\end{array}$ & $\begin{array}{c}\text { LONG - } \\
\text { I- }\end{array}$ & $\begin{array}{c}\text { OF } \\
\text { WELL, }\end{array}$ & $\begin{array}{c}\text { DATUM } \\
\text { (FT. }\end{array}$ & $\begin{array}{l}\text { DUCT - } \\
\text { ANCE }\end{array}$ & $\begin{array}{l}\text { DEG. C } \\
\text { DIS- }\end{array}$ & $\begin{array}{c}\text { PH } \\
\text { (STAND- }\end{array}$ & $\begin{array}{l}\text { TEMPER - } \\
\text { ATURE }\end{array}$ & $\begin{array}{c}\text { TEMPER - } \\
\text { ATURE }\end{array}$ \\
\hline \multirow[t]{2}{*}{ NUMBER } & DATE & TUDE & TUDE & $\begin{array}{l}\text { TOTAL } \\
\text { (FEET) }\end{array}$ & $\begin{array}{l}\text { ABOVE } \\
\text { NGVD) }\end{array}$ & $\begin{array}{c}\text { LAB } \\
\text { (US/CM) }\end{array}$ & $\begin{array}{l}\text { SOLVED } \\
\text { (MG/L) }\end{array}$ & $\begin{array}{c}\text { ARD } \\
\text { UNITS) }\end{array}$ & $\begin{array}{c}\text { AIR } \\
\text { (DEG C) }\end{array}$ & $\begin{array}{l}\text { WATER } \\
\text { (DEG C) }\end{array}$ \\
\hline & & & & (72008) & $(72000)$ & $(90095)$ & $(70300)$ & $(00400)$ & $(00020)$ & $(00010)$ \\
\hline
\end{tabular}

\begin{tabular}{|c|c|c|c|c|c|c|c|c|c|c|c|}
\hline NHW & 406 & $09 \cdot 01-89$ & 411023 & 0713544 & 178 & 159 & 169 & $\cdots$ & 6.6 & $\cdots$ & 17.5 \\
\hline NHW & 414 & $08-02-89$ & 411014 & 0713429 & 85 & 50 & 178 & $\cdots$ & 6.1 & $\cdots$ & 18.0 \\
\hline NHW & 419 & $08 \cdot 29 \cdot 90$ & 411036 & 0713418 & 52 & 35 & 312 & $\cdots$ & 6.1 & 26.0 & 15.5 \\
\hline NHW & 436 & $08-02-89$ & 410949 & 0713350 & 195 & 115 & 212 & - & 6.5 & $-\cdot$ & 15.0 \\
\hline NHW & 437 & $08-09-89$ & 411055 & 0713601 & 62 & 55 & '221 & $\cdots$ & 6.1 & $\cdots$ & 16.0 \\
\hline NHW & 451 & $07-26-89$ & 410932 & 0713258 & 220 & 120 & ${ }^{1} 257$ & $\cdots$ & 6.7 & $-\cdot$ & 12.5 \\
\hline NHW & 467 & $06-15-67$ & $41 \quad 1042$ & 0713415 & 53 & 20 &  & $\cdots$ & 6.8 & $\cdots$ & -- \\
\hline NHW & 504 & $08 \cdot 01-89$ & 411008 & 0713333 & 81 & 80 & 230 & $\cdots$ & 5.6 & -. & 17.0 \\
\hline NHW & 515 & $08-09-89$ & 410951 & 0713255 & 149 & 40 & 277 & $\cdots$ & 6.6 & -. & 20.0 \\
\hline NHW & 535 & $06-13 \cdot 91$ & 410927 & 0713432 & 163 & 154 & 148 & $\cdots$ & 7.5 & $\cdots$ & 12.0 \\
\hline NHW & 548 & $08-08-89$ & 410902 & 0713414 & 158 & 121 & 1216 & $\cdots$ & 6.5 & $\cdots$ & 13.0 \\
\hline NHW & 549 & $08-08-89$ & 410902 & 0713413 & 130 & 130 & 271 & $\cdots$ & 8.2 & $-\cdot$ & 20.0 \\
\hline NHW & 550 & $09-06-89$ & 410905 & 0713414 & 96 & 115 & 162 & $\cdots$ & 6.4 & -- & 13.5 \\
\hline NHW & 553 & $09-06-89$ & 410906 & 0713358 & 131 & 155 & 222 & $\cdots$ & 6.3 & $-\cdot$ & 17.0 \\
\hline NHW & 554 & $08 \cdot 01 \cdot 89$ & 410906 & 0713400 & 199 & 152 & 244 & $\cdots$ & 7.9 & $\cdot \cdot$ & 15.5 \\
\hline NHW & 556 & $08-09-89$ & 410910 & 0713358 & 222 & 168 & & $\cdots$ & 6.3 & $\cdots$ & 15.0 \\
\hline \multirow[t]{2}{*}{ NHW } & 557 & $08-08-89$ & $4109 \quad 17$ & 0713356 & 259 & 172 & 161 & $\cdots$ & 6.4 & $-\cdot$ & 14.5 \\
\hline & & $06-13 \cdot 91$ & & & & & 129 & $\cdots$ & 7.8 & -. & 11.5 \\
\hline NHW & 564 & $08-08-89$ & 410924 & 0713356 & 114 & 150 & $\leq 164$ & $\cdots$ & $<6.4$ & $\cdots$ & 16.5 \\
\hline NHW & 570 & $06-14-91$ & 410931 & 0713344 & 164 & 165 & 176 & $\cdots$ & 7.4 & $\cdots$ & 13.0 \\
\hline NHW & 614 & $08-09-89$ & 411023 & 0713622 & 102 & 43 & 1172 & $\cdots$ & $\cdots$ & $\cdots$ & 19.0 \\
\hline NHW & 629 & $08-28-90$ & 410928 & 0713416 & 33 & 137 & 195 & $\cdots$ & 6.2 & 23.0 & 14.5 \\
\hline NHW & 632 & $08-27-90$ & 410942 & 0713438 & 22 & 103 & 110 & $\cdots$ & 6.8 & 32.0 & 18.0 \\
\hline NHW & 658 & $08 \cdot 30-90$ & 411159 & 0713424 & 32 & 8 & 193 & $\cdots$ & 5.3 & 24.0 & 12.5 \\
\hline NHW & 668 & $08-22-89$ & 411216 & 0713356 & 111 & 20 & 490 & $\cdots$ & 7.1 & $\cdots$ & 17.0 \\
\hline NHW & 679 & $09 \cdot 06-89$ & 410934 & 0713550 & 195 & 125 & 140 & -- & 6.6 & $-\cdot$ & 15.0 \\
\hline NHW & 687 & $08-01-89$ & 411025 & 0713403 & 135 & 20 & 406 & $\cdots$ & 6.4 & -. & 17.5 \\
\hline NHW & 688 & $08-08 \cdot 89$ & 411016 & 0713400 & 130 & 45 & 275 & $-\cdot$ & 6.0 & $\cdots$ & 13.0 \\
\hline NHW & 695 & $08-08-89$ & $41 \quad 1003$ & 0713403 & 103 & 43 & 284 & $\cdots$ & 6.8 & $\cdots$ & 17.0 \\
\hline NHW & 706 & $09 \cdot 05-89$ & $41 \quad 1021$ & 0713503 & 244 & 100 & 201 & $\cdots$ & 6.5 & -. & 15.0 \\
\hline NHW & 708 & $09-05-89$ & 411056 & 0713515 & 102 & 55 & 139 & $\cdots$ & 6.2 & $\cdots$ & 14.5 \\
\hline NHW & 722 & $08-02-89$ & $41 \quad 10 \quad 18$ & 0713450 & 153 & 45 & 135 & $\cdots$ & 6.5 & - & 16.0 \\
\hline NHW & 738 & $08-02-89$ & 410953 & 0713452 & 147 & 135 & 163 & $\cdots$ & 6.6 & $\cdots$ & 17.5 \\
\hline NHW & 754 & $06-13 \cdot 91$ & 410929 & 0713307 & 360 & 124 & 250 & $\cdots$ & 6.9 & $\cdots$ & 14.5 \\
\hline NHW & 760 & $08-22-89$ & 410937 & 0713529 & 142 & 155 & 190 & $\cdots$ & 6.2 & $\cdot \cdot$ & 12.5 \\
\hline NHW & 768 & $08 \cdot 22 \cdot 89$ & 411019 & 0713600 & 131 & 80 & 166 & $\cdots$ & 6.9 & $\cdots$ & 14.0 \\
\hline NHW & 769 & $08-22 \cdot 89$ & 411109 & 0713536 & 100 & 55 & 185 & $\cdots$ & 6.1 & $\cdots$ & 21.0 \\
\hline NHW & 780 & $08-29-90$ & 411217 & 0713409 & 64 & 45 & 172 & $\cdots$ & 5.8 & 24.0 & 13.5 \\
\hline
\end{tabular}


WATER WH, whole water (unfiltered) sample;

fixed-endpoint titration for alkalinity;

Data Storage and Retrieval System code]

\begin{tabular}{|c|c|c|c|c|c|c|c|c|c|c|c|c|}
\hline & & & & & & BICAR - & ALKA- & & & & & \\
\hline & $\begin{array}{l}\text { HARD - } \\
\text { NESS }\end{array}$ & CALCIUM & $\begin{array}{l}\text { MAGNE- } \\
\text { SIUM }\end{array}$ & DIUM & $\begin{array}{l}\text { POTAS- } \\
\text { SIUM }\end{array}$ & $\begin{array}{l}\text { BONATE } \\
\text { WATER }\end{array}$ & $\begin{array}{l}\text { LINITY } \\
\text { WAT WH }\end{array}$ & SULFATE & $\begin{array}{l}\text { CHLO- } \\
\text { RIDE, }\end{array}$ & $\begin{array}{l}\text { FLUO- } \\
\text { RIDE, }\end{array}$ & BROMIDE & $\begin{array}{c}\text { SILICA, } \\
\text { DIS- }\end{array}$ \\
\hline OXYGEN, & TOTAL & DIS- & DIS- & DIs. & DIs- & WH IT & TOT FET & DIS- & DIs- & DIS- & DIS- & SOLVED \\
\hline DIS- & $(M G / L$ & SOLVED & SOLVED & SOLVED & SOLVED & LAB & LAB & SOLVED & SOLVED & SOLVED & SOLVED & $(M G / L$ \\
\hline SOLVED & AS & (MG/L & (MG/L & (MG/L & (MG/L & MG/L AS & (MG/L AS & (MG/L & (MG/L & (MG/L & $(M G / L$ & AS \\
\hline$(M G / L)$ & (ACO3) & AS (A) & AS MG) & AS NA) & AS $K$ ) & $\mathrm{HCO} 3$ & (ACO3) & AS $\mathrm{SO}_{4}$ ) & AS $C L$ ) & AS F) & AS BR) & S102) \\
\hline$(00300)$ & $(00900)$ & $(00915)$ & $(00925)$ & $(00930)$ & $(00935)$ & $(00449)$ & $(90410)$ & $(00945)$ & $(00940)$ & $(00950)$ & $(71870)$ & $(00955)$ \\
\hline
\end{tabular}

\begin{tabular}{|c|c|c|c|c|c|c|c|c|c|c|c|c|}
\hline$\cdots$ & $\cdots$ & $\cdots$ & $\cdots$ & -. & $\cdots$ & $\cdots$ & - & $\cdots$ & 22 & -. & $\cdots$ & $\cdots$ \\
\hline - & $\cdots$ & $\cdots$ & $\cdots$ & $\cdots$ & - & $\cdots$ & $-\cdot$ & - & 21 & .. & $\cdots$ & $\cdots$ \\
\hline 5.8 & 81 & 11 & 13 & 25 & 2.4 & 56 & 45 & 16 & 45 & 0.1 & 0.22 & 12 \\
\hline -. & - & $\cdots$ & $\cdots$ & $\cdots$ & - & -. & -. & -. & 27 & -. & $\cdots$ & $\cdots$ \\
\hline$\cdots$ & $\cdots$ & $\cdots$ & $\cdots$ & - & -. & -. & - & $\cdots$ & 32 & -. & -. & $\cdots$ \\
\hline -. & $\cdots$ & $\cdots$ & $\cdots$ & $\cdots$ & $\cdots$ & $\cdots$ & - & .. & 22 & .. & $\cdots$ & -. \\
\hline$\cdots$ & $\cdots$ & $\cdots$ & $\cdots$ & $\cdots$ & $\cdots$ & $\cdots$ & $-\cdot$ & $\cdots$ & 51 & $\cdots$ & $\cdots$ & $\cdots$ \\
\hline$\cdots$ & $\cdots$ & $\cdots$ & $\cdots$ & -- & $\cdots$ & $\cdots$ & $\cdots$ & $\cdots$ & 33 & $\cdots$ & $\cdots$ & $\cdots$ \\
\hline$\cdots$ & - & $\cdots$ & $\cdots$ & -. & .. & $\cdots$ & - & $\cdots$ & 42 & $\cdots$ & $\cdots$ & $\cdots$ \\
\hline$\cdots$ & $\cdots$ & $\cdots$ & $\cdots$ & $\cdots$ & $\cdots$ & 17 & 14 & $\cdots$ & 30 & -. & $\cdots$ & $\cdots$ \\
\hline$\cdots$ & $\cdots$ & $\cdots$ & $\cdots$ & - & - & -. & - & .. & 32 & .. & $\cdots$ & $\cdots$ \\
\hline$\cdots$ & $\cdots$ & $\cdots$ & $\cdots$ & $\cdots$ & - & $\cdots$ & $-\cdot$ & $\cdots$ & 46 & $\cdots$ & $\cdots$ & $\cdots$ \\
\hline$\cdots$ & $\cdots$ & $\cdots$ & $\cdots$ & -. & - &.- & $\cdots$ & $\cdots$ & 34 & $\cdots$ & -. & $\cdots$ \\
\hline$\cdots$ & $\cdots$ & $\cdots$ & $\cdots$ & -. & - & $\cdots$ & - & $\cdots$ & 38 & -. & - & $\cdots$ \\
\hline$\cdots$ & $\cdots$ & $\cdot \cdot$ & $\cdots$ & $\cdots$ & $\cdots$ & $\cdots$ & - & $\cdots$ & 34 & $\cdots$ & $\cdots$ & $\cdots$ \\
\hline$\cdot \cdot$ & $\cdots$ & $\cdots$ & -. & -. & -. & .. & -. & .. & 26 & -. & .. & -. \\
\hline -. & -. & $\cdots$ & $\cdots$ & $\cdots$ & - & $\cdots$ & -. & -. & 23 & $\cdots$ & $\cdots$ & $\cdots$ \\
\hline$\cdots$ & $\cdots$ & $\cdots$ & $\cdots$ & - & -. & 14 & 13 & - & 33 & $\cdots$ & -. & - \\
\hline$\cdots$ & $\cdots$ & $\cdots$ & $\cdots$ & $\cdots$ & $\cdots$ & $\cdots$ & $\cdots$ & $\cdots$ & 24 & $\cdot \cdot$ & $\cdots$ & $\cdots$ \\
\hline$\cdots$ & $\cdots$ & $\cdots$ & $\cdots$ & $\cdots$ & $\cdots$ & 14 & 12 & $\cdots$ & 35 & $\cdots$ & $\cdots$ & $\cdots$ \\
\hline -. & .. & -. & $\cdots$ & $\cdots$ & -. & -. & -. & -. & 24 & .. & -. & -. \\
\hline 2.4 & 41 & 7.3 & 5.6 & 19 & 3.9 & 62 & 49 & 4.5 & 32 & $<.1$ & .14 & 18 \\
\hline$\cdots$ & 30 & 5.3 & 4.1 & 9.0 & 2.5 & 49 & 40 & $<1.0$ & 11 & $<.1$ & .04 & 9.6 \\
\hline$\cdots$ & 38 & 9.2 & 3.7 & 20 & 1.6 & 24 & 19 & 13 & 33 & $<.1$ & .18 & 10 \\
\hline$\cdots$ & $\cdots$ & $\cdots$ & $\cdots$ & $\cdots$ & $\cdots$ & $\cdots$ & $\cdots$ & $\cdots$ & 110 & $\cdots$ & $\cdots$ & $\cdots$ \\
\hline$\cdots$ & $\cdots$ & $\cdots$ & $\cdots$ & $\cdots$ & $\cdots$ & $\cdots$ & $\cdots$ & $\cdots$ & 18 & -. & .. & - \\
\hline$\cdots$ & $\cdots$ & $\cdots$ & $\cdots$ & $\cdots$ & $\cdots$ & $\cdots$ & - & -- & 54 & $\cdots$ & -. & $\cdots$ \\
\hline$\cdots$ & $\cdots$ & $\cdots$ & $\cdots$ & $\cdot-$ & $\cdots$ & $\cdots$ & - & $\cdots$ & 40 & $\cdots$ & $\cdots$ & $\cdots$ \\
\hline$\cdots$ & $\cdots$ & -. & $\cdots$ & $\cdots$ & - & $\cdots$ & $\cdots$ & $\cdots$ & 37 & -. & -. & $\cdots$ \\
\hline$\cdots$ & $\cdots$ & $\cdots$ & $\cdots$ & $\cdots$ & $\cdots$ & $\cdots$ & $\cdots$ & $\cdots$ & 27 & $\cdots$ & $\cdots$ & $\cdots$ \\
\hline$\cdots$ & $\cdots$ & $\cdots$ & $\cdots$ & -. & -. & $\cdots$ & -. & -. & 17 & -. & $\cdots$ & .. \\
\hline - & $\cdots$ & $\cdots$ & $\cdots$ & -. & - & $\cdots$ & -. & -. & 16 & -. & $\cdots$ & $\cdots$ \\
\hline$\cdots$ & $\cdots$ & $\cdots$ & $\cdots$ & - & . & -. & -. & $\cdots$ & 19 & -. & $\cdots$ & $\cdots$ \\
\hline -. & $\cdots$ & $\cdots$ & $\cdots$ & $\cdots$ & -. & 20 & 17 & -. & 42 & -. & -. & -. \\
\hline$\cdots$ & $\cdots$ & $\cdots$ & $\cdots$ & $\cdots$ & $\cdots$ & $\cdots$ & - & $\cdots$ & 23 & - & $\cdots$ & $\cdots$ \\
\hline$\cdots$ & $\cdots$ & $\cdots$ & $\cdots$ & $\cdots$ & - & $\cdots$ & - & $\cdots$ & 21 & - & $\cdots$ & $\cdots$ \\
\hline -. & -. & $\cdots$ & - & - & -. & $\cdots$ & -. & 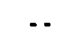 & 24 & $\cdots$ & $\cdots$ & -. \\
\hline -. & 31 & 6.5 & 3.7 & 17 & 2.8 & 21 & 19 & 8.9 & 32 & $<.1$ & .09 & 11 \\
\hline
\end{tabular}


Table 8.--Chemical and physical analyses of water from selected wells and springs--Continued

$[\cdots$, no data available; <, less than; WAT WH or WATER WH, whole water (unfiltered) sample;

IT, incremental titration for alkal inity; FET, fixed-endpoint titration for alkalinity; number in parentheses is USGS National Water Data Storage and Retrieval system code]

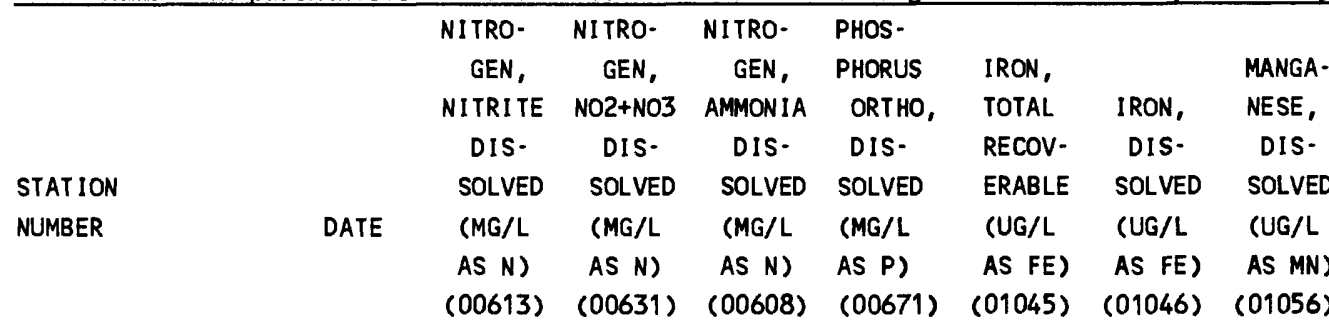

\begin{tabular}{|c|c|c|c|c|c|c|c|c|c|}
\hline NHW & 406 & $09-01-89$ & $<0.01$ & 0.365 & 0.02 & $\cdots$ & $\cdots$ & 200 & 40 \\
\hline NHW & 414 & $08-02-89$ & $<.01$ & 1.4 & .01 & $\cdots$ & $\cdots$ & 60 & 10 \\
\hline NHW & 419 & $08-29-90$ & $\cdots$ & 3.7 & $\cdots$ & 0.04 & $\cdots$ & 6 & $<1$ \\
\hline NHW & 436 & $08-02-89$ & $<.01$ & $<.01$ & .06 & -. & $\cdots$ & 5900 & 710 \\
\hline NHW & 437 & $08 \cdot 09-89$ & $<.01$ & 3.3 & $<.01$ & $\cdots$ & $\cdots$ & 60 & 60 \\
\hline NHW & 451 & $07-26-89$ & $<.01$ & .02 & .05 & $\cdots$ & $\cdots$ & 8100 & 370 \\
\hline NHW & 467 & $06 \cdot 15 \cdot 67$ & $\cdots$ & $\cdots$ & $-\cdot$ & $\cdots$ & 1 & -. & $\cdots$ \\
\hline NHW & 504 & $08-01-89$ & $<.01$ & 2.6 & $<.01$ & -- & -- & 30 & $<10$ \\
\hline NHW & 515 & $08-09-89$ & $<.01$ & .146 & .08 & $\cdots$ & $\cdots$ & 9700 & 520 \\
\hline NHW & 535 & $06-13-91$ & $-\cdot$ & $\cdots$ & $\cdots$ & $\cdots$ & $\cdots$ & $\cdots$ & $\cdots$ \\
\hline NHW & 548 & $08-08-89$ & $<.01$ & .147 & .04 & $\cdots$ & - & 3100 & 140 \\
\hline NHW & 549 & $08 \cdot 08-89$ & $<.01$ & .126 & .02 & $\cdots$ & $\cdots$ & 20 & $<10$ \\
\hline NHW & 550 & $09-06-89$ & $<.01$ & 1.0 & .02 & -- & $-\cdot$ & 90 & 20 \\
\hline NHW & 553 & $09-06-89$ & $<.01$ & .168 & .02 & $\cdots$ & $\cdots$ & 740 & 200 \\
\hline NHW & 554 & $08-01-89$ & $<.01$ & .024 & $<.01$ & $-\cdot$ & $\cdots$ & 40 & 550 \\
\hline NHW & 556 & $08-09-89$ & $<.01$ & 1.5 & .02 & $\cdots$ & $\cdots$ & 40 & 20 \\
\hline \multirow[t]{2}{*}{ NHW } & 557 & $08-08-89$ & $<.01$ & 2.4 & .02 & $\cdots$ & $\cdots$ & 70 & 30 \\
\hline & & $06-13-91$ & $\cdots$ & $\cdots$ & -. & $\cdots$ & .. & $\cdots$ & .. \\
\hline NHW & 564 & $08-08-89$ & $<.01$ & 2.7 & .02 & $\cdots$ & $\cdots$ & 50 & 140 \\
\hline NHW & 570 & $06-14-91$ & $\cdots$ & $\cdots$ & $\cdots$ & $\cdots$ & $\cdots$ & $\cdots$ & $\cdots$ \\
\hline NHW & 614 & $08 \cdot 09-89$ & $<.01$ & 1.4 & .02 & $\cdots$ & $\cdots$ & 20 & 50 \\
\hline NHW & 629 & $08-28 \cdot 90$ & -. & .400 & $\cdots$ & $<.01$ & - & 4100 & 860 \\
\hline NHW & 632 & $08 \cdot 27 \cdot 90$ & $\cdots$ & $<.1$ & $\cdots$ & .03 & $\cdots$ & 3900 & 1800 \\
\hline NHW & 658 & $08-30-90$ & $\cdots$ & 2.7 & $\cdots$ & $<.01$ & $\cdot-$ & 55 & 54 \\
\hline NHW & 668 & $08-22-89$ & $<.01$ & $<.01$ & .01 & $\cdots$ & $\cdots$ & 940 & 900 \\
\hline NHW & 679 & $09-06-89$ & $<.01$ & .612 & .02 & - & -. & 60 & 60 \\
\hline NHW & 687 & $08-01-89$ & $<.01$ & .02 & .02 & $\cdots$ & $\cdots$ & 14000 & 1000 \\
\hline NHW & 688 & $08-08-89$ & $<.01$ & 1.9 & .02 & $\cdots$ & $\cdots$ & 70 & $<10$ \\
\hline NHW & 695 & $08-08-89$ & $<.01$ & .117 & .140 & $\cdots$ & $\cdots$ & 9400 & 970 \\
\hline NHW & 706 & $09-05-89$ & .09 & 1.6 & .03 & $\cdots$ & $\cdots$ & 10 & 20 \\
\hline NHW & 708 & $09-05-89$ & $<.01$ & 2.0 & .02 & $\cdots$ & $\cdot \cdot$ & 170 & 10 \\
\hline NHW & 722 & $08-02-89$ & $<.01$ & .892 & $<.01$ & $\cdots$ & $\cdots$ & 30 & $<10$ \\
\hline NHW & 738 & $08 \cdot 02-89$ & $<.01$ & .534 & $<.01$ & $\cdots$ & $\cdots$ & 40 & 10 \\
\hline NHW & 754 & $06-13-91$ & $\cdots$ & $\cdots$ & $\cdots$ & $\cdots$ & $\cdots$ & $\cdots$ & - \\
\hline NHW & 760 & $08-22-89$ & $<.01$ & .834 & .02 & $\cdots$ & -. & 40 & 10 \\
\hline NHW & 768 & $08-22-89$ & $<.01$ & .02 & .03 & $\cdots$ & $\cdots$ & 540 & 610 \\
\hline NHW & 769 & $08 \cdot 22 \cdot 89$ & $<.01$ & $<.01$ & .02 & $\cdots$ & $\cdots$ & 1200 & 50 \\
\hline NHW & 780 & $08-29-90$ & $\ldots$ & 2.5 & $\because$ & .01 & $\therefore$ & 46 & 1 \\
\hline
\end{tabular}

${ }^{1}$ specific conductance is field value. 


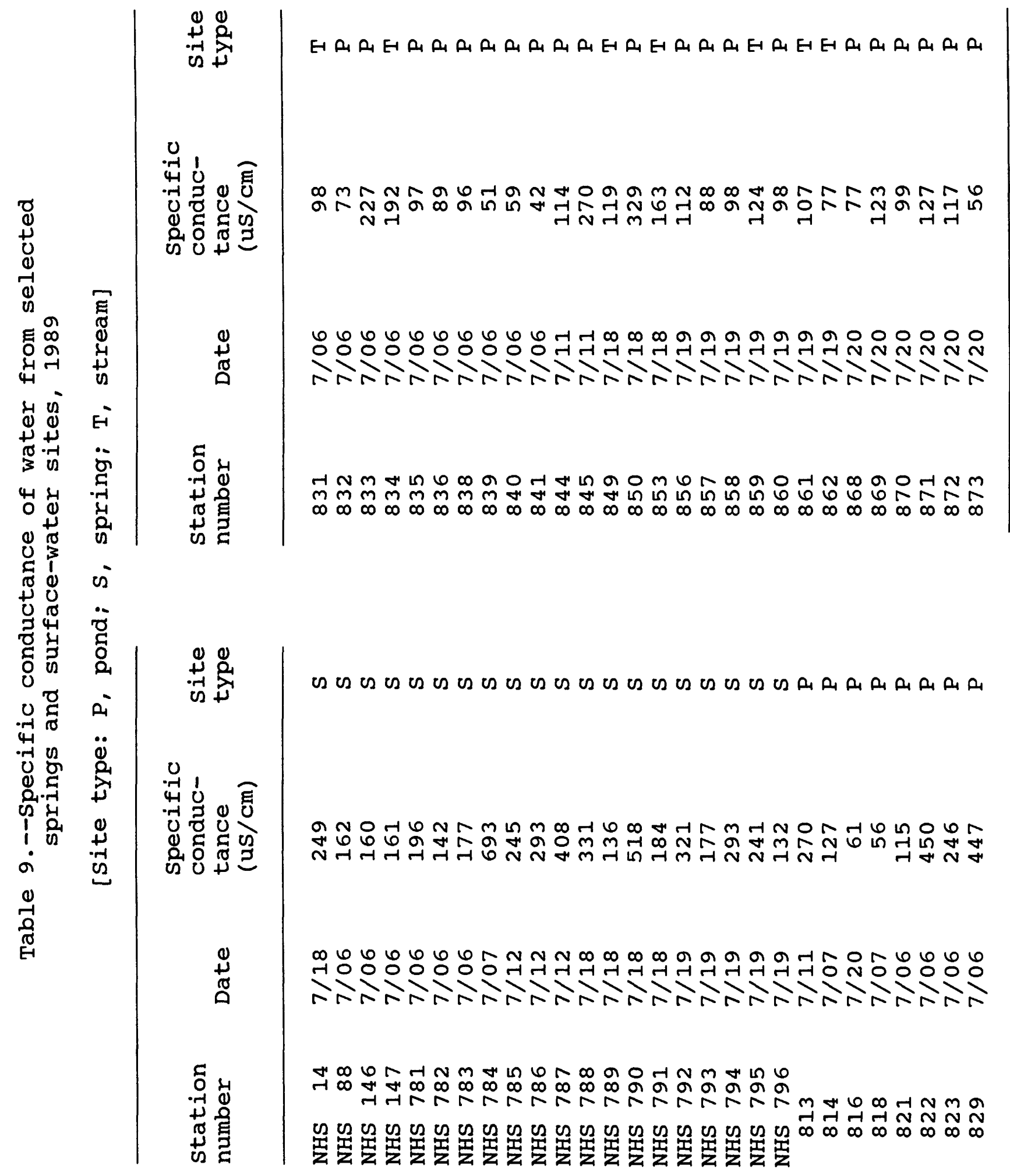




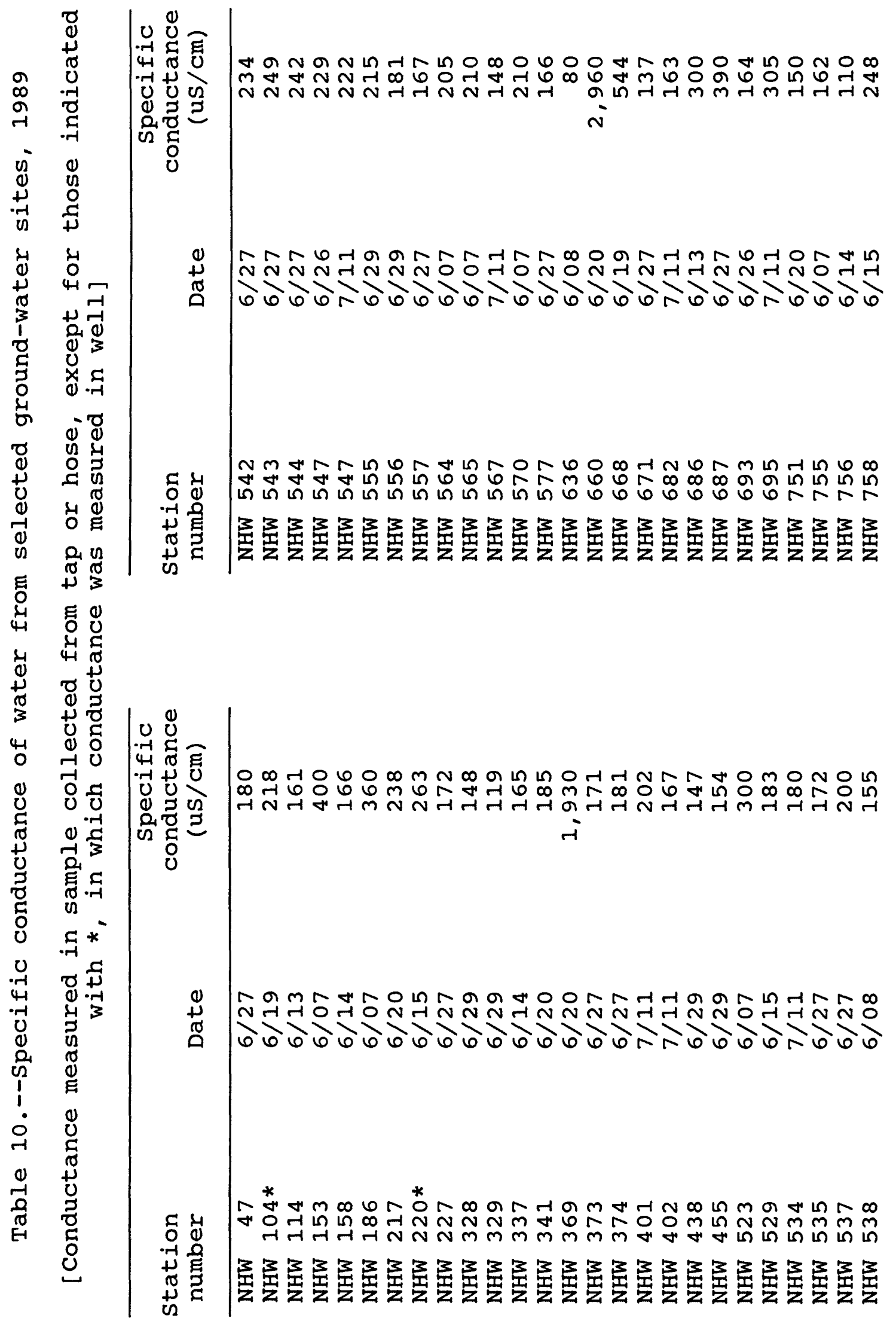


Tables 11-15. Rhode Island Department of Health analyses of Block Island public drinking-water sources

Tables 11-15 contain water-quality data for samples collected by the Rhode Island Department of Health (RIDOH) from the public-supply wells at the Block Island Water Company well field for the period 1976 to 1986 and from Sands Pond for the period 1976 to 1991. The tables also include data from Fresh Pond, a potential public water-supply source, for the period 1988 to 1991.

In the original tables from the RIDOH, the wells were referred to as water Company Wells \#1-\#6. The USGS' numbers for the water company wells are as follows:

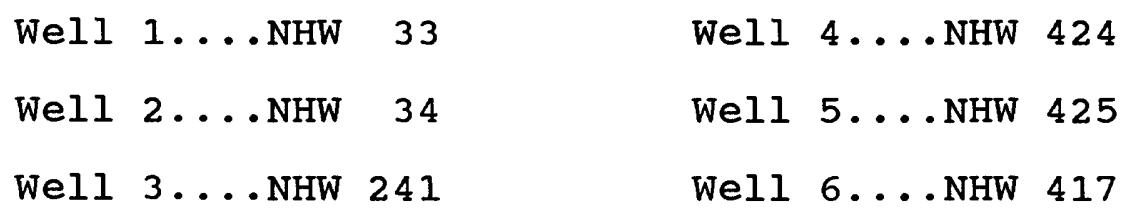

In some of the tables, there is a set of two years given for the sampling date instead of an exact date (example: 19841985). This period represents the State's fiscal year: July 1st of the first year to June 30th of the second year. The data in these tables are presented as they were published by the RIDOH. 







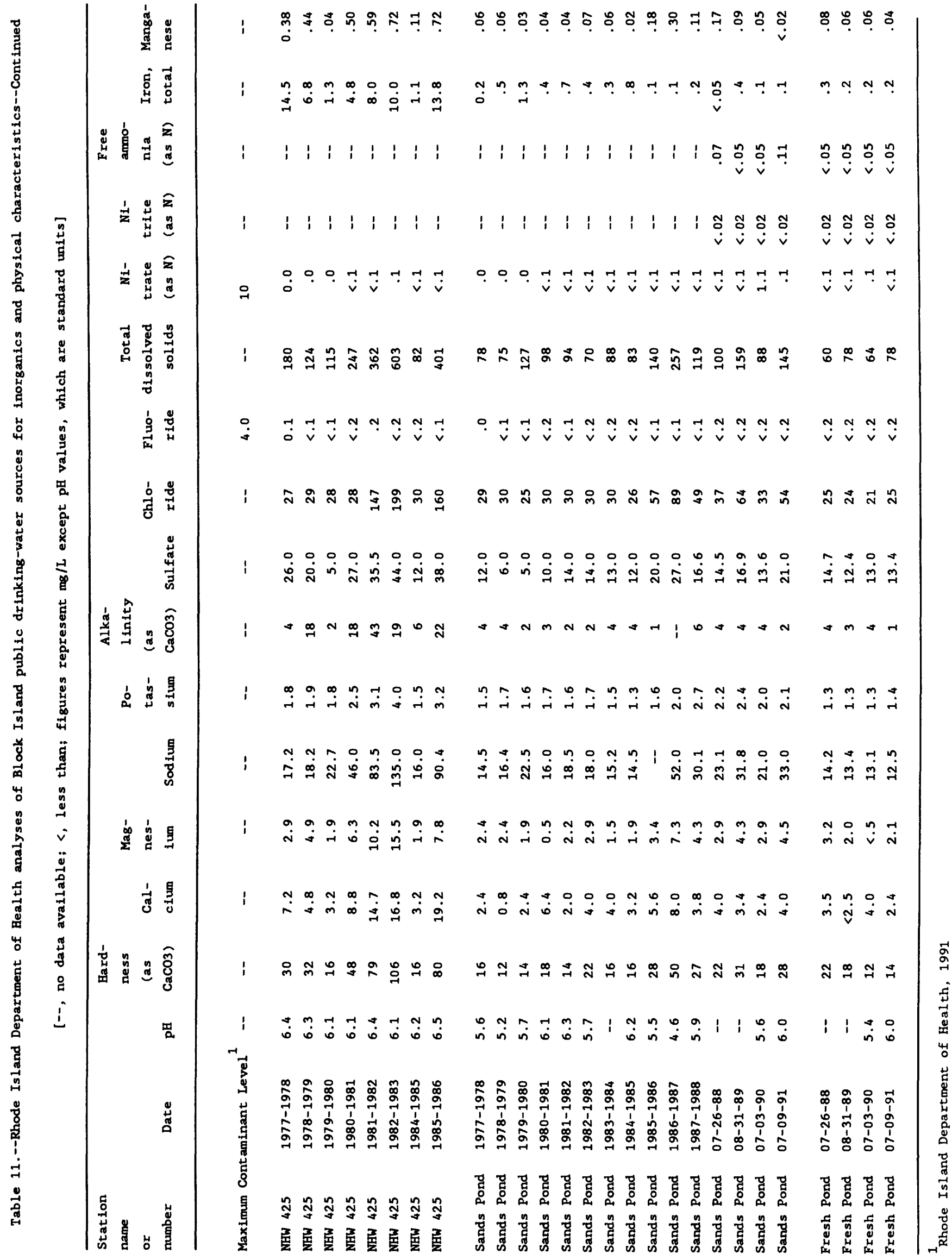




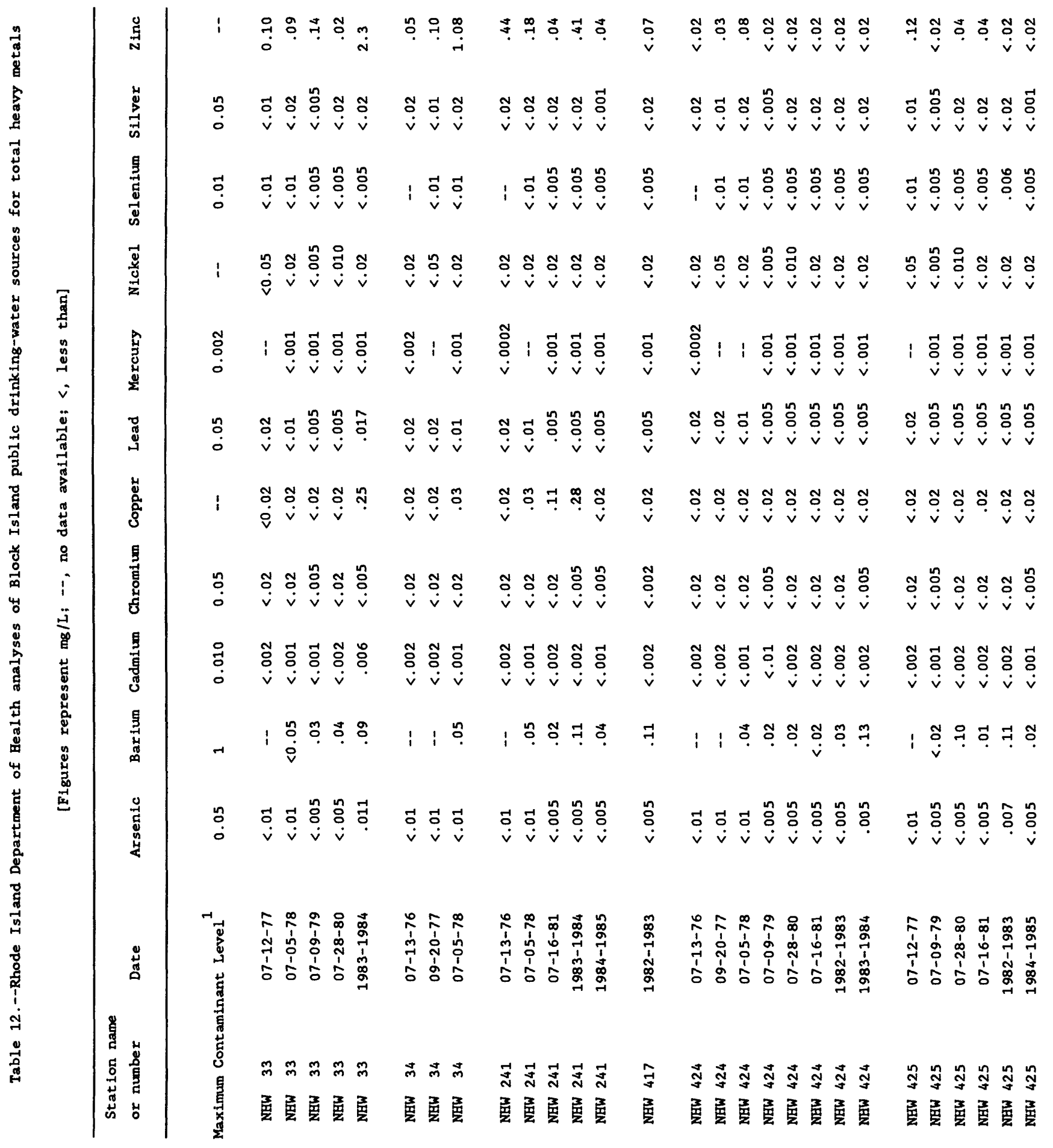









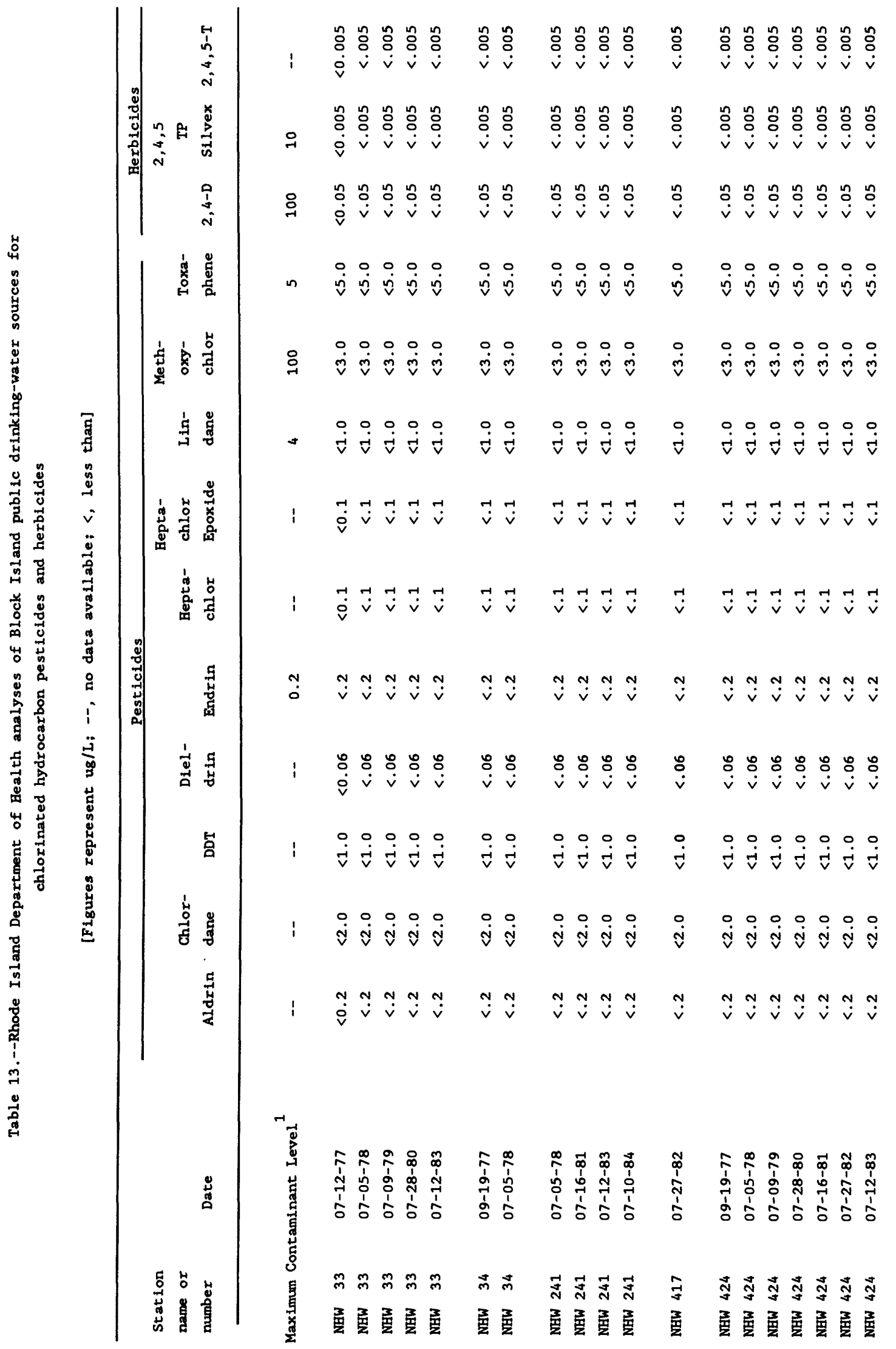




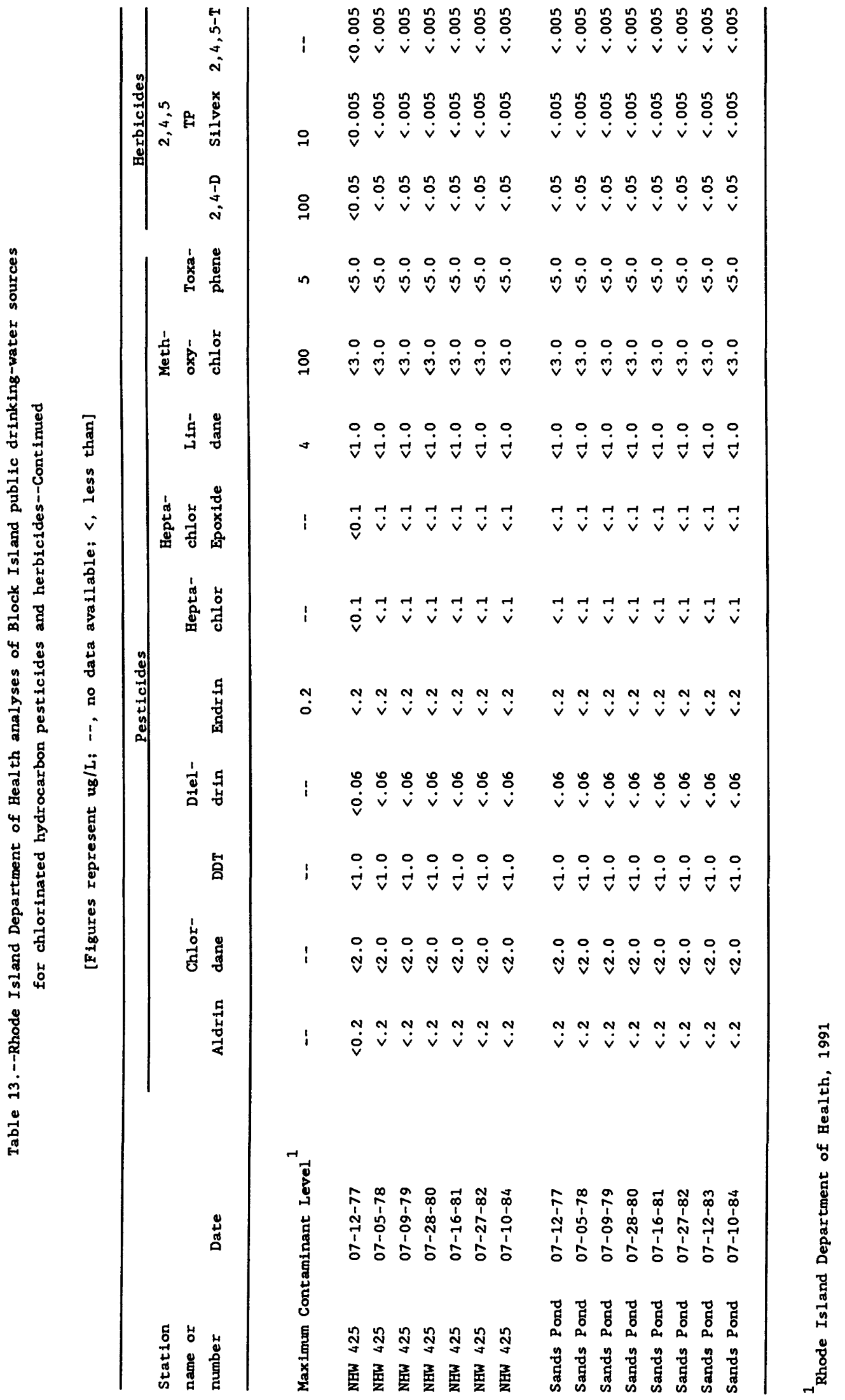


Table 14.--Rhode Island Dept. of Health analyses of Block Island public drinking-water sources for chloroform and trihalomethanes [--, no data available; ND, not detected]

\begin{tabular}{cccc}
\hline $\begin{array}{l}\text { Station } \\
\text { name or } \\
\text { number }\end{array}$ & Date & $\begin{array}{c}\text { Total } \\
\text { trihalo- } \\
\text { methanes } \\
(\mathrm{ug} / \mathrm{L})\end{array}$ & $\begin{array}{c}\text { Chloro- } \\
\text { form } \\
(\mathrm{ug} / \mathrm{L})\end{array}$ \\
\hline \multicolumn{7}{c}{} & & - \\
Maximum Contaminant Level & 100 & 1.20 \\
NHW 33 & $07-05-78$ & 1.30 & 3.10 \\
NHW 34 & $07-05-78$ & 3.80 & 1.60 \\
NHW 241 & $07-05-78$ & 1.60 & 1.20 \\
NHW 424 & $07-05-78$ & 1.90 & ND \\
NHW 425 & $07-05-78$ & ND & 58.90 \\
Sands Pond & $07-05-78$ & 85.90 & \\
\hline
\end{tabular}

${ }^{1}$ Rhode Island Department of Health, 1991 
Table 15.--Rhode Island Department of Health analyses of Block Island public drinking-water sources for radioactive constituents [--, no data available; <, less than]

\begin{tabular}{lll}
\hline & Gross & Gross \\
$\begin{array}{l}\text { Station name } \\
\text { or number }\end{array}$ & alpha & beta \\
(picocuries/ & (picocuries/ & iter) \\
\hline
\end{tabular}

\begin{tabular}{|c|c|c|c|c|}
\hline Maximum & \multicolumn{2}{|c|}{ n Contaminant Level ${ }^{1}$} & 15 & 50 \\
\hline $\begin{array}{l}\text { NHW } \\
\text { NHW } \\
\text { NHW } \\
\text { NHW } \\
\text { NHW }\end{array}$ & $\begin{array}{l}33 \\
33 \\
33 \\
33 \\
33\end{array}$ & $\begin{array}{l}07-11-77 \\
07-05-78 \\
07-09-79 \\
07-28-80 \\
07-12-83\end{array}$ & $\begin{array}{l}<0.53 \\
<.31 \\
<.60 \\
<.90 \\
3.3\end{array}$ & $\begin{array}{r}<1.52 \\
<1.56 \\
4.18 \\
<2.87 \\
5.5\end{array}$ \\
\hline $\begin{array}{l}\text { NHW } \\
\text { NHW }\end{array}$ & $\begin{array}{l}34 \\
34\end{array}$ & $\begin{array}{l}09-20-77 \\
07-05-78\end{array}$ & $\begin{array}{l}.69 \\
.61\end{array}$ & $\begin{array}{r}2.66 \\
<1.56\end{array}$ \\
\hline $\begin{array}{l}\text { NHW } \\
\text { NHW } \\
\text { NHW } \\
\text { NHW }\end{array}$ & $\begin{array}{l}241 \\
241 \\
241 \\
241\end{array}$ & $\begin{array}{l}07-05-78 \\
07-16-81 \\
07-12-83 \\
07-10-84\end{array}$ & $\begin{array}{l}<.31 \\
3.70 \\
3.3 \\
<3.0\end{array}$ & $\begin{array}{c}<1.56 \\
<5.90 \\
8.8 \\
<5.3\end{array}$ \\
\hline $\begin{array}{l}\text { NHW } \\
\text { NHW }\end{array}$ & $\begin{array}{l}417 \\
417\end{array}$ & $\begin{array}{l}07-24-81 \\
07-27-82\end{array}$ & $\begin{array}{c}<1.80 \\
4.6\end{array}$ & $\begin{array}{c}11.00 \\
4.4\end{array}$ \\
\hline $\begin{array}{l}\text { NHW } \\
\text { NHW } \\
\text { NHW } \\
\text { NHW } \\
\text { NHW } \\
\text { NHW } \\
\text { NHW }\end{array}$ & $\begin{array}{l}424 \\
424 \\
424 \\
424 \\
424 \\
424 \\
424\end{array}$ & $\begin{array}{l}09-20-77 \\
07-05-78 \\
07-09-79 \\
07-28-80 \\
07-16-81 \\
07-27-82 \\
07-12-83\end{array}$ & $\begin{array}{c}<.49 \\
<.31 \\
<.60 \\
<1.78 \\
3.60 \\
1.8 \\
3.2\end{array}$ & $\begin{array}{r}<1.55 \\
4.34 \\
<1.91 \\
<5.74 \\
<5.90 \\
<2.9 \\
<8.8\end{array}$ \\
\hline $\begin{array}{l}\text { NHW } \\
\text { NHW } \\
\text { NHW } \\
\text { NHW } \\
\text { NHW } \\
\text { NHW } \\
\text { NHW }\end{array}$ & $\begin{array}{l}425 \\
425 \\
425 \\
425 \\
425 \\
425 \\
425\end{array}$ & $\begin{array}{l}07-11-77 \\
07-05-78 \\
07-09-79 \\
07-28-80 \\
07-24-81 \\
07-27-82 \\
07-10-84\end{array}$ & $\begin{array}{l}<.53 \\
<.31 \\
<.60 \\
<1.78 \\
<1.80 \\
<3.6 \\
<3.0\end{array}$ & $\begin{array}{r}1.91 \\
<1.56 \\
3.91 \\
7.12 \\
6.80 \\
<12.0 \\
<5.3\end{array}$ \\
\hline $\begin{array}{ll}\text { Sands } & \mathrm{P} \\
\text { Sands } & \mathrm{P} \\
\text { Sands } & \mathrm{P} \\
\text { Sands } & \mathrm{P} \\
\text { Sands } & \mathrm{P} \\
\text { Sands } & \mathrm{P} \\
\text { Sands } & \mathrm{P} \\
\text { Sands } & \mathrm{P}\end{array}$ & $\begin{array}{l}\text { Pond } \\
\text { Pond } \\
\text { Pond } \\
\text { Pond } \\
\text { Pond } \\
\text { Pond } \\
\text { Pond } \\
\text { Pond }\end{array}$ & $\begin{array}{l}07-11-77 \\
07-05-78 \\
07-09-79 \\
07-28-80 \\
07-16-81 \\
07-27-82 \\
07-12-83 \\
07-10-84\end{array}$ & $\begin{array}{r}<.53 \\
.96 \\
<.60 \\
<.45 \\
<1.80 \\
. .96 \\
1.0 \\
<1.5\end{array}$ & $\begin{array}{c}4.21 \\
2.16 \\
4.34 \\
3.51 \\
5.70 \\
4.7 \\
<2.9 \\
3.3\end{array}$ \\
\hline
\end{tabular}

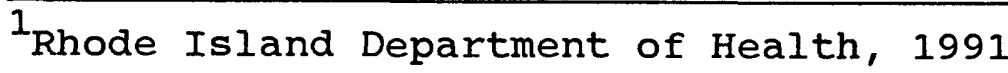

